\title{
Summary of Research for the Inertial Confinement Fusion Program at Los Alamos National Laboratory
}

\author{
Compiled by \\ David C. Cartwright \\ Principal ICF Program Manager
}

\begin{abstract}
DISCLAIMER
This report was prepared as an account of work sponsored by an agency of the United States Government. Neither the United States Government nor any agency thereof, nor any of their employees, makes any warranty, express or impliod, or assumes any legal liability or responsibility for the accuracy, completeness, or usefulnese of any information, apparatus, product, or process disclosed, or represents that its use would not infringe privately owned rights. Reference bercin to any specific commercial product, process, or service by trade name, trademark, manufacturer, or otberwise does not necessarily constitute or imply its endorsement, recommendation, or favoring by the United States Government or any agency thereof. The views and opinions of authors expresied herein do not necessarily state or reflect those of the United States Govemment or any agency theroof.
\end{abstract}




\section{CONTENTS}

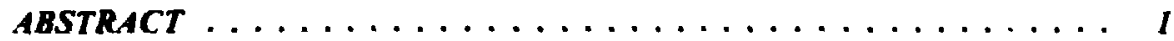

Inertial Confinement Fusion Overview . . . . . . . . . . . . . . 3

High-Energy-Density Plasma Physics with Lasers . . . . . . . . . . . 8

The KrF Laser: The Advance Toward Shorter Wavelengths . . . . . . . . . . . 23

Diagnosis of Laser-Driven Implosions . . . . . . . . . . . . . . . . 34

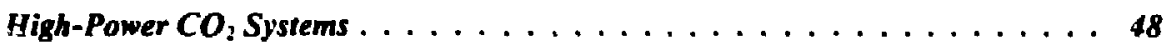

Target sabrication for Inertial Confinement Fusion Research . . . . . . . . . 72

Heavy-Ion Fusion . . . . . . . . . . . . . . . so

ABSTRACTS OF ICF SUPPPORTED

RESEARCH PUBLICATIONS SINCE 1982. . . . . . . . . . . 9I 


\title{
Summary of Research for the
}

\section{Inertial Confinement}

Fusion Program

\section{at}

\section{Los Alamos}

National Laboratory

\author{
compiled by \\ David C. Cartwright \\ Principal ICF Program Manager
}

\begin{abstract}
The information presented in this report is a summary of the status of the Inertial Confinement Fusion (ICF) program at the Los Alamos National Laboratory as of February 1985. This report contains material ori the existing high-power $\mathrm{CO}_{2}$ laser driver (Antares), the program to determine the potential of $\mathrm{KrF}$ as an ICF driver, heavy-ion accelerators as drivers for ICF, target fabrication for ICF, and a summery of our understanding of laser-plasma interactions. A classified companion report cuntains material on our current understanding of capsule physics and lists the contributions to the Laboratory's weapons programs made by the ICF program. The information collected in these two volumes is meant to serve as a report on the status of some of the technclogic:l components of the Los Alainos ICF program rather than a detailed review of specific technical issues.
\end{abstract}




\title{
Inertial Confinement Fusion Overview
}

\author{
by David C. Cartwright
}

$\mathbf{T}$ he accomplishment of energy gain through nuclear fusion in laboratory experiments will require the solution of a number of interesting, although very complex, scientific and technological problems. Both the Inertial Confinement Fusion (ICF) and Magnetic Fusion Energy (MFE) programs have been somewhat overly optimistic in their initial evaluations of the difficulties involved in controlling nuclear fusion. In both programs, most of the major problems were identified only after the construction and operation of a new and usually more powerful experimental facility. Researchers in both the ICF and MFE programs are now trying to build solid technical foundations for their prograns in order to be able to predict results under different experimental conditions. Los Alamos completed construction of the Antares $\mathrm{CO}_{2}$ laser facility in December 1983 and placed it on operational status for experiments in ICF. Antares, the world's largest operational laser, is the first in a series of new. higher intensity ICF drivers and is the successor to the smaller ( $\leq 10-\mathrm{kJ}$ ) Helios eight-beam $\mathrm{CO}_{2}$ laser facility at Los Alamos. The two others in the US are Nova, at Lawrence Livermore National Laboratory (LLNL), to be operational in the spring of 1985. and PBFA-II at Sandia National Laboratories (SNL), to begin operation in the fall of 1987. These three new facilities will permit experimentation with ICF targets under conditions of temperature and pressure that more closely simulate those required for an energy gain target.

The ICF mission statement for the Los Alamos National Laboratory is as follows:

The Los Alamos ICF program is one of the main efforts by the Department of Energy (DOE) to evaluate the scientific feasibility of inertially confined fusion, using intense lasers or particle hearns to compress and heat small masses of deuirium-tritium fuel to thermonuclear burn conditions. The goals of the national program are:

- to support nuclear weapons; physics research, and

- to do research on the potential of inertial fusion for energy production.

Key technical elements within the ICF program are:

- the design and confirmation of performance of jiselfllled targets requiring minimum input energ;, and

- the development of a laboratory driver suitable jor driving such targets (at an acceptable cost).

Whether addressing weapons applications or possibie long-term potential as an energy source, there is no significant difference in the short-term program. In the longer term, important scientific and engineering problems would have to be addressed before ICF could be considered for commercial electricai sower generation. Since the primary source of the funding is the DOE defense programs, the weapon physics goals will continue to receive emphasis throughout this decade.

Since Antares has been in operarion for more than a year, and Helios was operational for 5 years before Antares, it is appropriate to review our present understanding of ICF physics based on $\mathrm{CO}_{2}$ laser drivers. The purpose of this technical review is to present our level of knowledge of the coupling of $\mathrm{CO}_{2}$ laser energy to the target, acquired by experiments with both the Helios and Antares laser facilities. In the subsequent chapters, th: scientific and technological progress that has been made in the fields of plasma physics, capsule physics, materials fabrication, $\mathrm{CO}_{2}$ and $\mathrm{KrF}$ laser systems, and heavy-ion fusicn (in support of the ICF program at Los Alainos) will be reviewed. A review of the Los Alamos program to study the physics of laser fusion at short wavelength (that is $1 / 4 \mu \mathrm{m}$ ) will be presented in a future publication. 


\section{GENERAL REQUIREMENTS FOR ICF}

Inertial confinement fision attempts to mimic on a minianure scale the physics of a thermonuclear weapons device. $A$ fuel of deuterium and tritium (DT) is heated rapidly to temperatures high enough to promote fusion reactions in the fuel, and at the same time, the fuel is compressed to densities large enough to facilitate reaction of a large fraction of the fuel before cooling by hydrodynamic expansion. However, the efficient use of the DT fuel in ICF is much more difficult than in a nuclear weapon.

The difficulty arises from the requirement to contain (in the laboratory) the eneray released by the fusion reaction. This can be accomplished only if the yield is sufficiently low, which implies a small mass of fuel. For small fuel masses to support thermonuclear burn, in which the energy produced by fusion reaction is used to sustain the burn, the fuel must be condensed to high density. Specifically, to trap the energy of the a-particles produced in the DT fusion reaction, the areal density (pR) must be equal to or greater than a certain constant C. That is. a fundamental scaling parameter for all ICF is the product of fuel density $p$ and the radius $R$ of the volume containing the fuel. It can be shown that if the product $\rho R$ is equal to a constant. then the density to which the fuel must be compressed increases as the reciprocal of the square root of the fuel mass, that is. $\rho \approx$ $1 / \sqrt{ }$ M. Achieving the required compression without expending excessive energy from the driver is the basic requirement of ICF.

Figure I shows schematically the various steps required to convert the incident laser energy into hydrodynamic compression of the fuel. Information on steps (a) and (b) is being obtained using laboratory drivers such as Helios and Antares, but data concerning steps (c) and (d) will require new and more powerful drivers. Keep in mind that the objective is to achieve net energy gain from the fusion reaction. Thus, it is important to achieve as large an energy conversion efficiency as possible at each step in the sequence. Because we know that ICF will work at some $\operatorname{lar}_{i}=$ drive energy, it is important to determine energy conversion efficiencies and energy requirements for each individual step as the figure of merit for judging the potential for success for laboratory drivers such as $\mathrm{CO}_{2}$. The remainder of this introduction summarizes the scientific processes governing each step shown in the linkage of laser energy to fuel compression in Fig. 1. What is treated as an assertion at this level is explained, and qualified, in the articles that follow.

\section{CHARACTERISTICS OF CO -IRRADIATED TARGETS}

When high-intensity laser radiation $\left(<10^{15} \mathrm{~W} / \mathrm{cm}^{2}\right)$ strikes a solid surface. a plasma is formed. The infrared radiation $(10.6 \mu \mathrm{m})$ produced by the $\mathrm{CO}_{2}$ laser is absorbed by the plasma through a collective process known as resonant absorption. This process. which we have come to understand primarily through theoretical work. converts almost all of the absorbed laser energy into energetic electrons. The measured absorption for flat surfaces is about $40 \%$, and refraction of the incident light in the plasma reduces the absorption to about $30 \%$ for curved surfaces. The energetic electrons produced during the resonant absorption process approach temperatures of $80 \mathrm{keV}$. This distribution of so-called "hot electrons" can exist superimposed on a second "cold" distribution because there are very few collisions in the low-density corona formed around the target. Experiments show that hot-electron temperature increases with laser intensity as I.4.

Because all of the energy absorbed from the laser beam initially resides in the hot electrons, it is mandatory to understand the subsequent transport of the electrons in order to predict how energy can be delivered to other portions of the target. Very large electric and magnetic fields develop, for conditions characteristic of $\mathrm{CO}_{2}$-laserdriven targets, as a result of the large spatial gradients in temperature and density present in the plasma. The magnetic fields are confined to within a few hundred micrometers of the surface and may exceed a megagauss. Consequently, the magnetic forces dominate the motion of most electrons, and their transport is not described by mathematical solutions of the diffusion equations. The self-consistent theoretical treatment of plasma motion under the influence of an intense laser radiation field has been a major accomp!shment of the theorists in this program.

For $\mathrm{CO}_{2}$ laser intensities exceeding $2 \times 10^{15} \mathrm{~W} / \mathrm{cm}^{2}$. the self-generated magnetic fields become large enough to prevent hot electrons from returning to their origirs on the surface defined by the critical plasma density $\left(10^{19}\right.$ $\mathrm{cm}^{-3}$ for $10.6-\mu \mathrm{m}$ radiation). As a consequence of this 


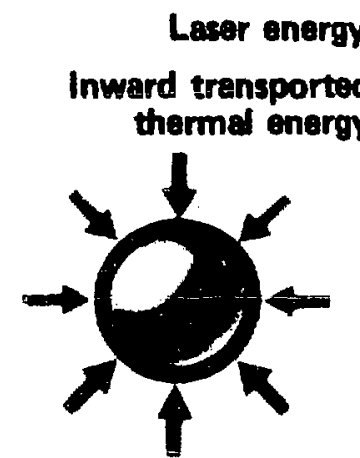

Atmosphere Formation

Laser or particle beams rapidly heat the surface of the fusion target

forming a surrounding plesma envelope.

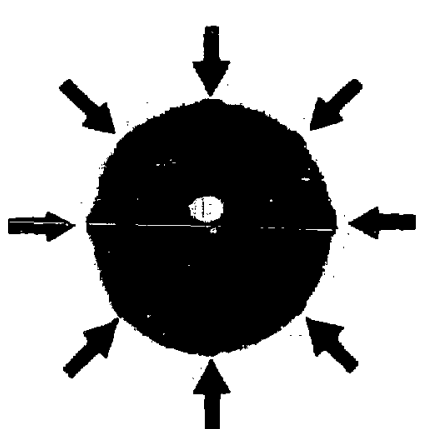

Compression

Fuel is compressed by rocket-like blowoff of the surface material.

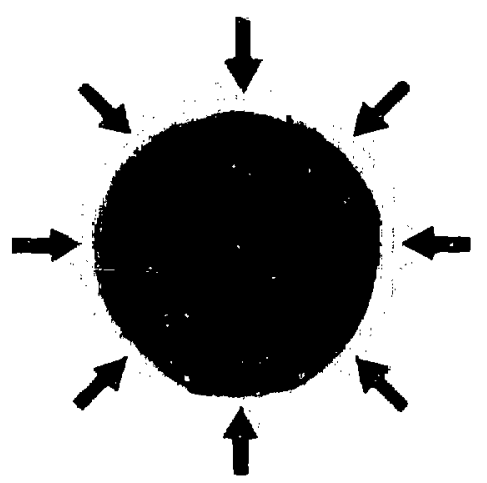

Ignition

With the final driver pulse, the full core reaches $1000-10,000$ times

liquid density and ignites at $100,000,000^{\circ} \mathrm{C}$.

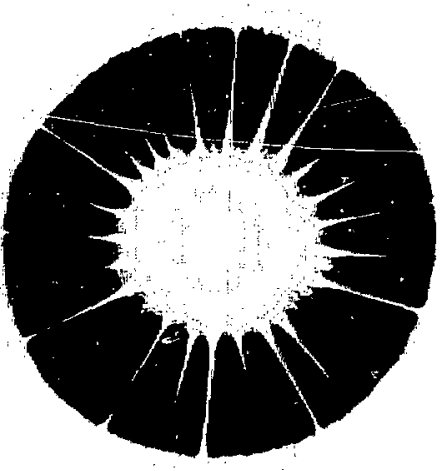

Burn

Thermonuclear burn spreads repidly through the compreased fuel, yiolding many times the driver input energy.

(a)

(b)

(c)

(d)

Fig. 1. Steps in the conversion of incident laser energy into hydrodynamic work compression on the fuel. Source is Ref. 1. 
magnetically enforced charge separation, ions are accelerated away from the target surface. This ion "blowoff' is not efficient in driving an implosion of the fuel because the momentum per unit energy of the ions is low and represents an energy loss for targets. Elaborate target geometries have been designed that attempt to efficiently recover the energy carried by the fast ions.

For good hydrodynamic coupling of momentum to the fuel, the ablation of a large mass at the (low) velocity set by the thermal energy content of the ablator material is required. To minimize the energy needed to compress the fuel, it is necessary to ininimize preheat to an energy level that places the fuel on an adiabat that does not greatly exceed $\mathrm{T} \approx 5 \mathrm{keV}$ at the time of maximum compression. Further refinements are possible only if the central portion of the fuel is elevated to ignition temperatures by properly timed shock collisions. This central portion of the fuel can then trigger heating of the remainder of the fuel by local deposition of the $\alpha$-particles released in the DT reaction.

In the Los Alamos program, we have atternpted to capitalize on the great operational advantages of the $\mathrm{CO}_{2}$ laser, the most efficient and inexpensive high-power laser available. To develop the next generation driver will require several hundred million dollars of investment. For this reason, all conceivable ways of using the absorbed energy in whatever form it might appear in the target (electrons, ions, $x$ rays, and so forth) are being carefully and systematically explored. These efforts were reviewed by external panels of experts in 1982 and 1983. In the most recent review, the possible use of the large internal magnetic fields to provide insulation from preheat by hot electrons was suggested. Work in this regard has progressed to the development and computational testing of a novel target that combines advantages of both ICF and MFE by inclusion of magnetic fields for inhibition of electron thermal conduction. This target concept is now being tested using the Antares laser.

\section{CONTRIBUTIONS BY ICF TO THE WEAPONS PROGRAMS}

Almost since its inception, the ICF program has been viewed as a potentially significant contributor to the understanding of the physics of nuclear weapons design. Numerous studies over the years have attempted to define a role for ICF in weapons physics, identifying the potential for equation-of-state measurements, opacity measurements, thermonuclear burn studies once ignition was achievable, and large-scale $x$-ray vulnerability facilities once yields approaching $200 \mathrm{MJ}$ were obtaineu.
The contributions from the ICF program to the weapons program are summarized below.

- One of the greatest contributions from ICF is its unique computer code capability.

- The national ICF pregram would be critical to the US defense program in the event of a comprehensive test ban treaty (CTBT) or a lowered threshold (LTBT) on nuclear testing. New drivers such as light ions (PBFA-II), lasers (for example, KrF), or HEdriven concepts could provide a means of exercising and maintaining design capability under a CTBT as well as a means of addressing the feasibility of fusion itself.

- Improvements in material fabrication developed within ICF program have been important ingredients in testing certain $x$-ray concepts for the Strategic Defense Initiatives (SDI) program.

- The Antares laser facility is a unique source of $x$-ray and microwave radiation and can be of direct use in studying the response of materials to this intense radiation. A future laboratory fusion facility could indsed study vulnerability, lethality, and weapons effects, particularly in the event of a CTBT.

- The physics of rare-gas halide lasers studied in the ICF program is highly beneficial to SDI considerations of laser weapons and their effects.

In addition to the early-identified results cited above, both the weapons and ICF programs have benefited from sharing and exchanging personnel. During its 12 ycars at Los Alamos, the ICF program has attracted many outstanding technical people to the Laboratory who might not otherwise have come and helped them develop in technical areas of direct interest to the weapons program. Now the Laboratory benefits from their contributions to other Laboratory activities.

\section{THE FUTURE}

Ovelr 10 years of research have taught us a great deal about ICF on a laboratory scale, and it is certainly more difficult than originally envisioned. The inevitable inefficiencies in all the steps between providing incident energy and igniting a fuel have plagued the program. However, there still exists the certainty that ICF works well on some energy scale, and in our quest to provide unlimited energy by control of fusion, the workability of ICF should not be overlooked. We are still far from ary practical use, but the promise warrants a sustained national effort. For at least the remainder of this decade, 
we should fully utilize the potential of the research facilities and the technology base we have already estabisshed.

Should our attempts to devise a $\mathrm{CO}_{2}$ laser target that will achieve high energy gain fail, technology devclopment programs for both a new laser system $(\mathrm{KrF})$ as well as an alternative to all lasers, a heavy ion-beam accelerator, are in progress at Los Alamos. The purpose of the $\mathrm{KrF}$ program at Los Alamos is to understand the science and technology required to develop an efficient, rep-ratable, low-cost, short-wavelength source of intense radiation that can be scaled to meet future ICF driver needs. In the case of the heavy-ion program, the purpose is to work with the other participants to identify and begin resolution of the key technical issues associated with a heavy-ion ICF driver. These programs are not so much intended to provide a large target-shooting capability as to establish the cost scaling of these particular systems for potential megajoule-level systems. The research activities on these two advanced ICF drivers are described in this review.

\section{REFERENCE}

1. H. G Ahlstrom. Physics of Laser Fusion, Vol II, Diagnostics of Experiments on Laser Fusion Targets at $L L N L$, Lawrence Livermore National Laboratory, January 1982, p 5. 


\title{
- High-Energy-Density Plasma Physics With Lasers
}

\author{
by David W. Forslund and Philip D. Goldstone
}

\section{$\mathbf{T}$} his section is a preliminary draft being prepared for publication in Los Alamos Science. It is meant primarily as an introduction to the complex plasma processes which occur in the process of laser fusion.

To successfully fulfill the promise of inertial fusion, an extremely wide variety of physical phenomena involved with the flow of matter and energy must be controlled with considerable precision. Experimental and theoretical studies of the deposition of laser light and transfer of that energy to particles have uncovered many new phenomena that have altered many simple models for use in fusion and that have greatly enhanced our understanding of extremely high-energy-density plasmas such as most probably exist in the atmospheres of white dwarfs or in galactic nuclei. albeit on a much shorter time and space scale. We must understand the physics of matter from $10^{-5}-10^{3} \mathrm{~g} / \mathrm{cm}^{3}$ over distances of $10^{-5}-10^{0} \mathrm{~cm}$ and times of $10^{-15}-10^{-8} \mathrm{~s}$ with particle energies ranging from $0.1 \mathrm{eV}$ to $10^{6} \mathrm{eV}$ and magnetic fields ranging from none at all to $10^{3} \mathrm{~T}$. The physics involved is nearly collisionless plasma physics including the effects of spontaneously generated magnetic fields, tiigh-density collisional physics. the atomic physics of weakly to highly ionized materials, and their effects on transport of photons of all energies as well as particles. Frequently the processes are far from equilibrium and require elaborate rate equations to reasonably accurately describe the effects.

The biggest problem for inertial fusion in general, and laser fusion in particular. has been the mechanism of deposition of laser light energy into the target and subsequent transport of that energy to the ablation surface. The very properties of lasers that allow them to produce extremely high power and intensity (which is necessary for fusion) can work against a desirable form of energy deposition. In particular, the wave nature of the light, the high coherence, and narrow bandwidth all contribute to peculiar collective effects in the deposition that reduce the efficiency of several of the above steps. In other words, these highly organized properties of the laser tend to drive the hot plasma in the target far from thermodynamic equilibrium with potentially serious consequences. Although these processes are not favorable for laser fusion, they enable one to achieve extremely high-enegy-density plasmas with very high specific energy per particle. In particular, laser fusion with $\mathrm{CO}_{2}$ lasers at $10 \mu \mathrm{m}$ is dominated by such effects and allows the physicist to view such exotica in all their complexity.

In this section, we discuss a number of these unusual collective processes as found in high-power $\mathrm{CO}_{2}$ lasertarget physics and briefly discuss some of the possible relationships with processes taking place in other physical systems far removed in scale from ICF research. The reader should note that shorter wavelength laser-plasma interactions tend to be more collisional, and the collective processes discussed here are generally less dominant.

The collective mechanisms we discuss initially result in the production of energetic electrons by the absorption of laser radiation in the target corona. The hot-electron energy then "cascades" into a number of energy flow channels (magnetic fields, acceleration of energetic ions, bremsstrahlung emission, heating of a dense "thermal" plasma, and microwave emission). Whereas much of our understanding of absorption processes has been theoretical because of the limited number of unambiguous experimental signatures of these processes at $10 \mu \mathrm{m}$, our understanding of energy flow through this hierarchy has largely been empirical, with theory providing explanation of our observations and a basis for predicting scaling of these phenomena. ${ }^{1}$ In many cases, these theoretical explanations have provided new hypotheses to test experimentally as well as new insights into how the energy flow might be altered. 


\section{THEORETICAL TOOLS}

We will begin by describing the methods we have used to study these processes and a lir le about how they have developed and improved over the years. The inertial fusion program has had a well-balanced program with considerable strength in both the theoretical and experimental areas. Because of the extreme complexity of the physics, it has been at the forefront in developing new theoretical tools as well as experimental techniques. The phenomena of interest change over time scales of picoseconds with the entire experiment occurring in about a nanosecond. Spatial resolution required to study the physics of targets measured in hundreds of micrometers to millimeters typically approaches $10 \mu \mathrm{m}$ (the wavelength of the $\mathrm{CO}_{2}$ light). Since much of the truly microscopic (submicrometer, subpicosecond) phenomena cannot be readily measured, but strongly influence the macroscopic behavior, computer simulation is relied on heavily to couple the microscopic phenomena to macroscopic observables. The WAVE code is a 2D particle simulation code that solves Maxwell's equations and the relativistic Newton's laws for the particles in the selfconsistent three-component electric and magnetic fields. It typically advances $10^{6}$ particles on a grid of $10^{5}$ cells for $10^{4}$ time steps to determine the processes that absorb the laser light and transport the energy from the lowdensity, hot-plasma regions to the denser regions. Only a portion of the physical problem can be modeled because one must limit the time step to a small fraction of the laser period and the grid size to the distance light travels in one time step. Consequently, the time that can be covered is only a few picoseconds and the distance is only about 10 wavelengths of light. Because of the limited time and space scale, the boundary conditions and initial conditions for fields and particles are unknown and must be estimated. Much of the skill in using the code involves guessing the right boundary and initial conditions that appear to be consistent with themselves, with hydrodynamic calculations, and with experiment.

An implicit form of the code, called VENUS, ${ }^{2}$ has enabled one to greatly increase the time step and grid size (at the expense of some high-frequency phenomena) to consider realistic spatial scales, which allows the study of the central role of self-generated magnetic fields in electron transport and fast-ion emission. After their development by the ICF program. these new-generation codes rapidly spread to the magnetic fusion and space physics communities. Although quantum mechanical and atomic physics process are not included in these codes, they can accurately describe the fully developed strong turbulence that can occur in laser-plasma interactions, limited only by the computer resources. To better utilize the results of these codes, we test and evaluate various models to obtair scaling laws that can be reliably extrapolated into new regimes. For example, simplified models of the propagation and absorption of light in the plasmas have been verified as to their accuracy and range of validity with the WAVE code. In the last decade, the improvement in scale of problems accessible has increased by a couple of orders of magnitude because of improvements in computer hardware speed and in numerical algorithms (VENUS). This has greatly increased our understanding of the physical processes as discussed below.

\section{EXPERIMENTAL TOOLS}

In addition to the theoretical tools that have greatly aided our insight into the complex processes in these plasmas, a wide variety of experimental tools and techniques have provided the basic empirical information against which the theoretical models must be compared in an iterative process. Overall energy balance can be obtained by measuring the nonabsurbed light directly or by calorimetrically measuring all of the ion and $x$ radiation energy emitted from the target. Some hot electrons escape the target and can be detected with electron spectrometers: however, the bulk of the electron energy is observable primarily by measurement of the bremsstrahlung radiation from several kiloelectron volts to large fractions of a megaelectron volt, where possible with subnanosecond time resolution. Typically, electron temperatures of hundreds of kiloelectron volts are observed. ${ }^{3}$ A variety of instruments can be used to determine the spectra of accelerated ions.

The heated target material emits soft $x$ rays that are detected by multichannel soft $x$-ray spectrometers utilizing filtered vacuum $x$-ray (photo) diodes, along with ultrafast oscilloscopes developed in the nuclear weapons program, to provid- time resolution of about 200 ps. Spatially restricting the area viewed by the soft $x$-ray diodes enables measurement of the brightness temperature as well as the color temperature of a specific part of the target surface. This is provided by multichannel $x$-ray collimators. The small targets used in the IC:" program require that these collimators be made of precisely machined pinholes of approximately $150 \mu \mathrm{m}$ diameter. aligned with respect to each other and the target to an accuracy of $25 \mu \mathrm{m}$ using optical techniques. 
Optical and $x$-ray emission can be spectrally resolved. or imaged using simple optical cameras or pinhole cameras, and either images or spectra can be time resolved to tens of picoseconds using image-converter streak camer:s. The detailed atomic physics of the plasma corona. often far out of local thermodynamic equilibrium, can be examined using high-resolution $x$-ray and xuv spectrometers to observe the spectra from the corona. Details of the spectra such as line broadening and line shapes can be used together with detailed atomic physics models as a probe of the plasma conditions surrounding the ions of interest; this can be particularly useful in spectroscopically examining the conditions of the imploded fuel. The pinhole cameras, soft $x$-ray coliimators, and $x$-ray spectrometers must be close to the target and must survive the intense $x$-ray and particle debris "blast" from each shot.

Imaging oi the target plasma in $x$-rays characteristic of some transition of intcrest in the target or corona, for example a $\mathrm{K}$-line resulting from a hot-electron-caused inner shell vacancy, enables the experimenter to track the flow of electron energy.

Detailed microfabrication technology is used to produce complex targets. with specific materials placed at strategic locations, so that their $x$-ray emission can be used as a "tracer" to determine the amount, or the time. of energy flow to these locations, as well as allowing detailed examination of plasma conditions from a welldefined region, without having to average over all densities and temperatures achieved in the plasma.

The availability of such a wide range of measurement technologies. together with the detailed ability to design experiments by microscopic modification of the targets. has allowed us to develop a detailed empirical base against which the theoretical picture of the processes involved can be compared.

\section{COMPARISON WITH EXPERIMENT}

- The insight gained from WAVE/VENUS simulations and verification of various models has led to the improvement in various physics packages in the $2 \mathrm{D}$ radiation now/hydrodyna nics code LASNEX and to a better choice of input $c$ snditions to the code. LASNEX then is used to model the hydrodynamics, electron transport, and radiation flow and possible thermonuclear burn in the target. Sometimes it can be used to evaluate models by observation of their effect on the macroscopic target behavior. LASNEX has postprocessing packages such as TDG which allow it to generate directly outputs from various diagnostic instruments such as pinhole photographs, streak camera images, bremsstrahlung spectra. ion emission, and soft $x$-ray emission. Since the code also calculates the source spectra. it can be used to assist in the deconvolution of, for example, the electron temperature from the hard $x$-ray bremsstrahlung spectrum or the compressed-fuel properties from the $x$-ray pinhole images and $x$-ray spectra. By simultaneous modeling of a variety of phenomena on a given target shot, the use of the code greatly enhances one's confidence in understanding the target behavior. Frequently there has to be an iteration ir LASNEX input conditions to match the experimental data. In other cases suggestions can be made to modify existing diagnostics or develop new ones to search for specific predicted signatures of physical phenomena. From this iteration process, new target concepts have been developed in an attempt to better utilize the energy flow.

\section{LASER LIGHT TRANSPORT AND ABSORPTION}

In large laser systems, the light must travel from the last large optical surface a long distance through a nearvacuum to the target. The intensity at the target is controlled to a large extent by the focal properties of the final optical element and is adjusted for direct-drive laser fusion to achieve nearly uniform illumination at the target. The initial illumination uniformity is not maintained, however, because as the target heats up, hot plasma is blown off. Because the index of refraction , of a plasma depends on density as $n=1-\omega_{p}^{2} / \omega^{2}$, where $\omega_{p}^{2}=$ $4 \pi \mathrm{ne}^{2} / \mathrm{m}$ is the square of the local plasma frequency, $\omega$ is the laser frequency, and $\mathrm{n}$ is the electron density, the propagation of light in this plasma is altered from what it is in a vacuum. Linearly, the light may refract and not strike the target where it was originally. Nonlinearly, the light may break up into filaments because of localized heating of the plasma or the finite pressure of the light itself. This may cause local regions of light to be much more intense than others, which may degrade the symmetry of the implosion or increase the energetic electron generation.

A important first step in the overall energetics of laser fusion is the determination of how much of the incident light is absorbed in the target. Absorption is measured in variety of ways including measuring the scattered light 
from the target and measuring the integrated ion blowoff kinetic energy from the target. Figure 1 shows the absorption on spheres of various sizes as a function of intensity as measured by ion calorimetry. Typically, at the lower intensities shown at the left, the absorption is about $30 \%$, whereas at high intensities it can exceed $60 \%$.

What are the mechanisms of absorption and how can we identify which ones are actually operative in laser target experiments? Although inferences can be made from a variety of experimental data, most of the information on absorpion processes have come from computer simulations and analytic theory, which have been iterated to reproduce the experimental data base. The simplest form of absorption of laser light results from collisions of electrons oscillating in the laser electric field with the background ions. This process, known as inverse oremsstrahlung, works by randomly scattering the oscillating electrons off the iors. Thus, coherent osciliation energy is converted into random energy. By equating the dissipated light energy, $\nu E^{2} / 8 \pi$, to the heating rate,

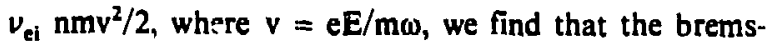
strahlung absorption frequency is $\nu=v_{\mathrm{el}} n / n_{\mathrm{c}}$, where $n_{\mathrm{c}}$ is the density at which $\omega_{p}=\omega$. The absorption length for light then is $\mathrm{c} / \mathrm{v}$. Since the electron-ion collision frequency $v_{\mathrm{ei}}$ varies as $\mathrm{v}^{-3}$, inverse bremsstrahlung preferentially heats low-velocity electrons and thus keeps the plasma close to thermodynamic equilibrium. If $L$ is the density scale height, then significant absorption occurs for $\omega \mathrm{L} / \mathrm{c}$ $\approx 1$. If we balance the absorbed energy with the electron heat flux, we see that significant absorption only occurs for

$$
\mathrm{I}<5 \times 10^{14} \mathrm{Z} \mathrm{L}(\mathrm{cm}) / \lambda^{4}
$$

where $\mathbf{Z}$ is the icn charge state, $\mathbf{L}$ is the plasma scale length in $\mathrm{cm}$ and $\lambda$ is the laser wavelength in micrometers. Thus, for $\mathrm{L} \approx 1 \mathrm{~mm}$ and $\mathrm{Z}=79$, inverse bremsstrahlung is negligible for the $\mathrm{CO}_{2}$ wavelength of $10 \mu \mathrm{m}$ at intensities above $10^{12} \mathrm{~W} / \mathrm{cm}$. Therefore, some form of collective $\Delta$ bsorption that is somewhat less desirable than

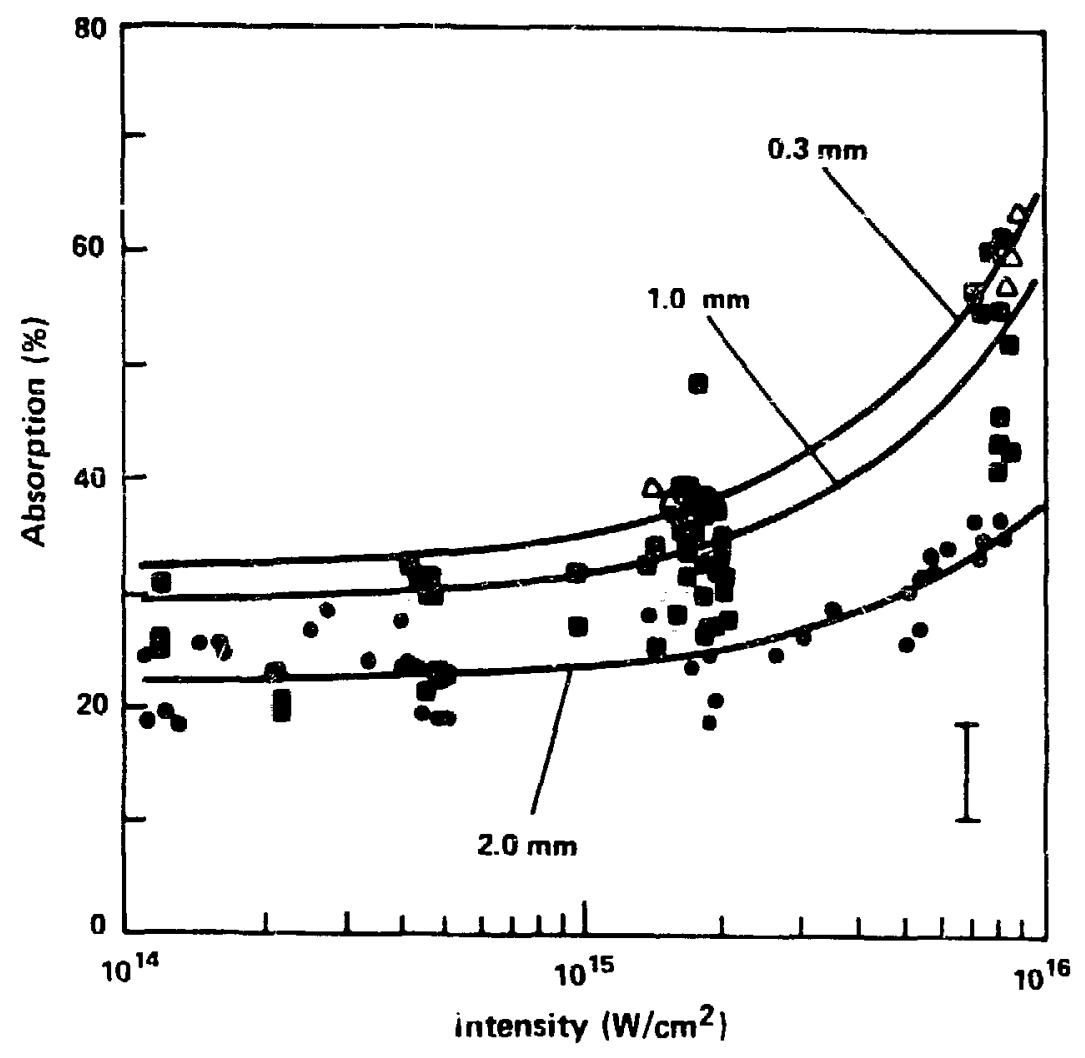

Fig. 1. Absorption as rneasured by ion calorimetry of the expanding plasma for spheres of varying size. 
inverse bremsstrahiung must be relied on. At wavelengths less than $1 \mu \mathrm{m}$ (for example, the 0.25- $\mu \mathrm{m}$ light of $\mathrm{KrF}$ lasers), inverse bremsstrahlung is very important and appears experimentally to dominate the absorption process, although, some undesirable forms of collective absoiption are still present to a modest extent.

An important quantity that affects the collective absorption piocesses is the pressure of the incident light wave, the so-called ponderomotive force. $\mathbf{F}=\omega_{\mathrm{p}}{ }^{2} / \omega^{2} \nabla|E|^{2}$. It is a low-frequency force proportional to the iight intensity and the ratio of the plasma density to the critical density of the incident light. For example. at $10^{16} \mathrm{~W} / \mathrm{cm}^{2}$, the pressure of the iight at its reflection point is about 5 Mbars! This large force is able to distort the flow of expanding plasma at low densities and is responsible for most of the instabilities induced by the incident radiation in the underdense plasma.

Because of this strong ponderomotive force, two basic mechanisms of collective absorption have been identified as important in all laser-plasma interactions and particularly important for $\mathrm{CO}_{2}$ lasers.

The first is called resonant absorption, 3.6 in which the electric field of an obliquely incident laser beam can linearly couple to a longitudinal plasma wave in the lowdensity expanding plasma. For a wave with an incident angle $\theta$. the electromagnetic wave is reflected from the region $n=n_{c} \cos ^{2} \theta$ but may still tunnel to the resonant matching point as shown in Fig. 2. At the resonant point, the component of the electric vector along the density gradient induces time-dependent density fluctuations at the local plasma frequency. This acts as a source of plasma waves and exiracts energy from the incident electromagnetic wave. The conversion efficiency depends sensitively on the incident angle and the scale length between these two points. For angles of incidence of the order of $20^{\circ}$, the scale length must be less than a wavelength of light to obtain an absorption of greater than 20\%. In fact, the large ponderomotive pressure gradient of the reflecting light weve and the locally generated plasma wave produce a sharp density gradient in that region, which allows resonant absorption be an effective process. In Fig. 3, we show the sharp density gradient from a WAVE simulation that extends to densities far above the critical density. An important experimental identification of resonant absorption at high laser intensities is from the large second harmonic emission of light produced. The nonlinear coupling of the density fluctuations of the plasma wave to the incident light produces the second harmonic through the current $j(2 \omega) \approx v \delta \mathrm{n}$. Its intensity is proportional to the incident light wave intensity and the plasma wave intensity. The scaling of the second harmonic emission is a strong function of incisent laser intensity. At low intensity the $2 \omega$ emission is proportional to $I^{2}$. At high intensity, where

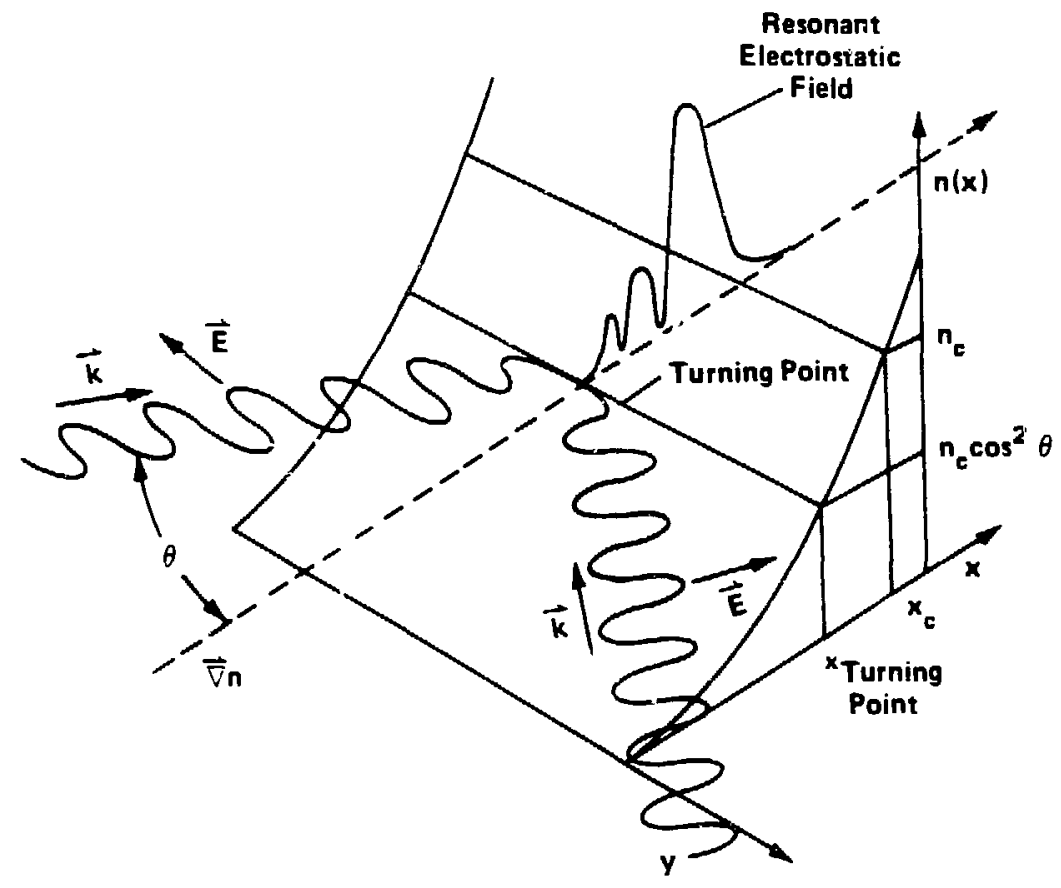

Fig. 2. The rescnance absorption process 


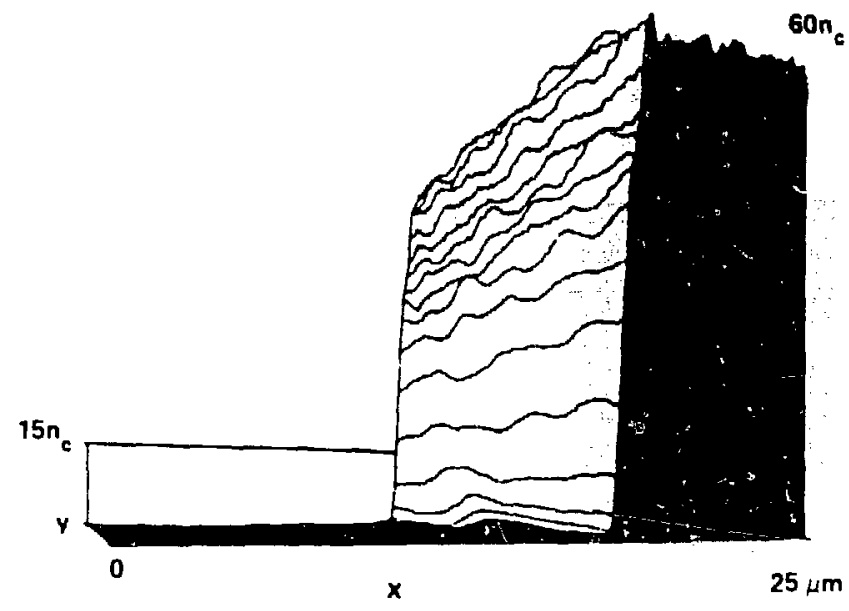

Fig. 3. Density gradients obtained from the WAVE code.

(a)

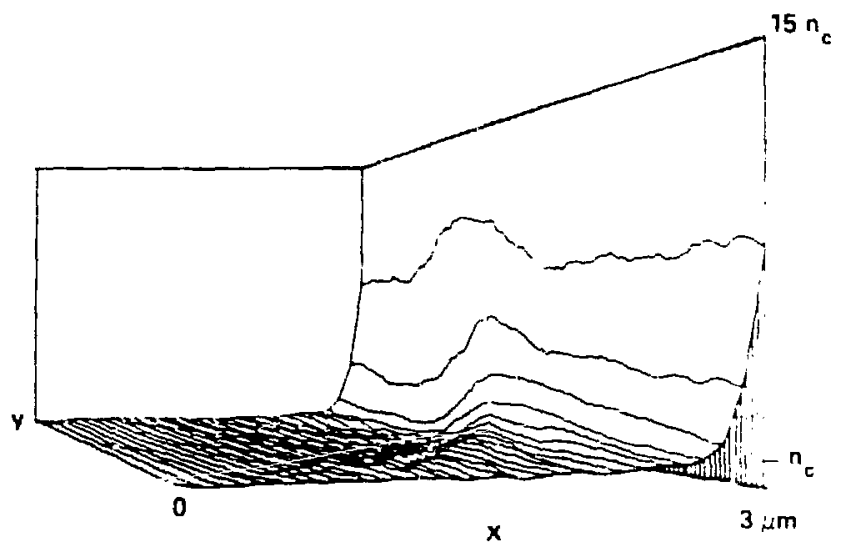

(b)

on saturates because of nonlinearities, the $2 \omega$ emission is proportional to I.

\section{WAVE BREAKING}

Once the enzrgy is placed into the plasma wave, it is quickly dissipated into the plasma by a number of mechanisms. Because of the extremely high intensity of the laser, the primary way in which it is absorbed is by a process known as wave breaking. In this process a few electrons can gain energies of hundreds of kiloelectron volts in less than one laser cycle (about $10^{-14} \mathrm{~s}$ ) over distances of less than a micrometer as they are accelerated by the plasma wave. The energy they gain is proportional to the electric field of the plasma wave and its width. Since the width is narrower because of the steepened profile. the electron energy is reduced over the one that would occur in some gentle profile. ${ }^{8}$ Although the acceleration process is quite coherent, it is observed in computer modeling to have a small random change from cycle to cycle, which injects stochasticity into the distribution, resulting in a near-Maxwellian hot-electron distribution.9 Although nearly all of the electrons at the critical density can be heated by this process. only a small fraction of electrons above the critical-density surface is hot. Thus, we typically see a two-component electron plasma consisting of a large nun "ir of "cooler" electrons with a small number of energetic electrons over a broad energy spectrum that carry most of the heat. Because the equilibration time between the two components is quite long, this situation persists as long as the laser is at high power. An example of the distribution of electrons at high density is shown in Fig. 4. A major uncertainty in the 


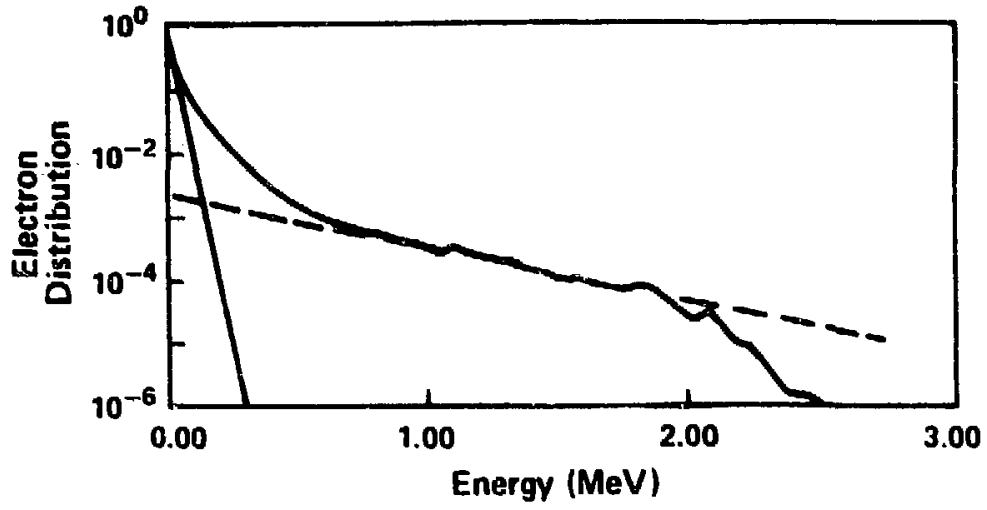

Fig. 4. The heated-electron distribution from a WAVE simulation.

simulations is what the cool background temperature is. Since it depends on the radiative cooling processes, the expansion of the solid material, ionization rates. and so forth, it cannot be determined self-consistently with the WAVE code but must be modeled with LASNEX. Nevertheless, this relatively simple theory has resulted in a reasonable match between theory anci experiment ${ }^{8}$ as to the magnitude of the hot-electron temperature and the nearly constant absorption of $20-30 \%$ that is observed from $10^{14}-10^{15} \mathrm{~W} / \mathrm{cm}^{2}$. The most spectacular confirmation of this process is the observation in experiment and simulations of high harmonics of the incident laser light.? In fact, experiments as illustrated in Fig. 5 have shown more than 35 harmonics of the incident light in the scattered-light spectrum, confirming the extreme noniinearity of the interaction in the steepened density gradient. These high harmonics are a signature of the extremely anharmonic character of the acceleration seen by the electrons in the intense coherent resonant abscrption plasma wave ${ }^{10}$ as shown in Fig. 6. The plasma wave in the steep gradient sees a strongly spatially varying restoring force proportional to $\omega_{\mathrm{p}}^{2}$. The maximum harmonic content theoretically expected is up to the plasma frequency of the upper density shelf. The experiment then implies that the plasma wave is secing densities up to 1000 times the critical density, or essentially solid density.

In recent years, however, experiments at higher intensity and more extensive calculations have indicated that this very steep gradient may not last for a long time.

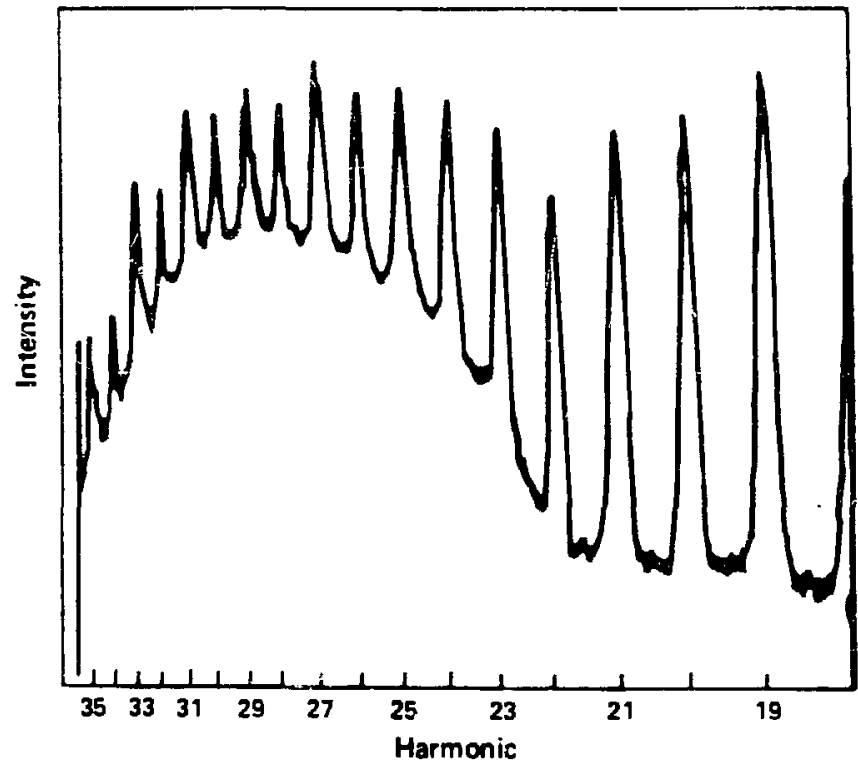

Fig. 5. Harmonics of the incident $10-\mu \mathrm{m}$ light produced by nonlinear coupling mechanisms in the steep plasma gradient. 


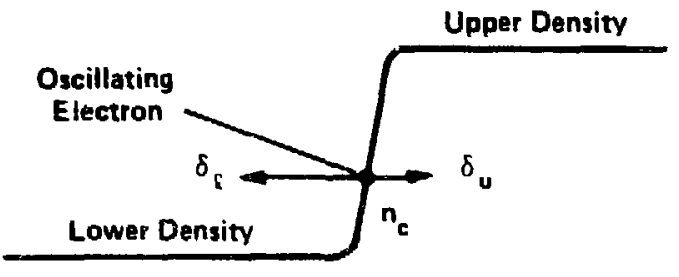

(a)

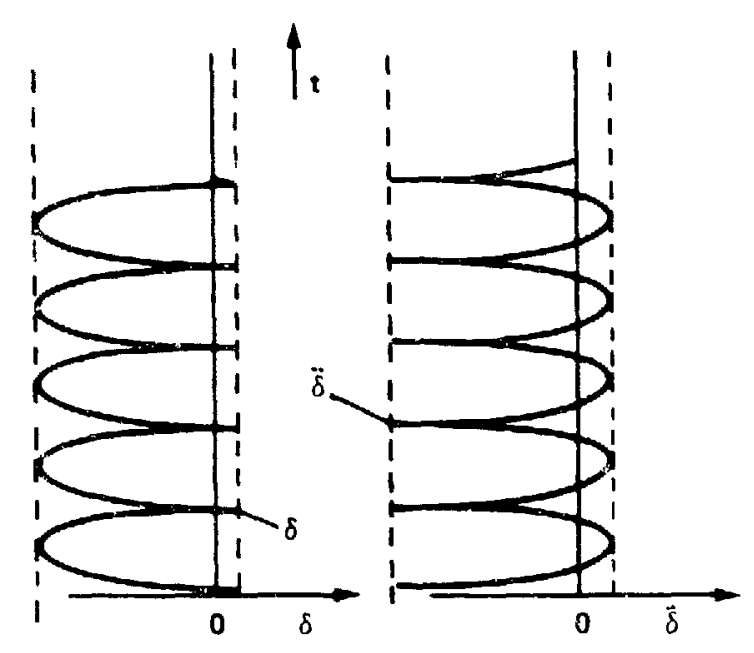

(b)

When the high harmonics are time resolved using "lirafist optical streak cameras, they are observed to last only during the rise time of the laser pulse. about 200 ps. This is in part due to the expansion of the plasma outward from solid density so that the plasma wave no longer sees the strong acceleration at those high densities. In addition. the nearly constant absorption coefficient observed at low intensity is observed to increase dramatically above about $10^{15} \mathrm{~W} / \mathrm{cm}^{2}$ as shown in Fig. 1. This suggests that an additional absorption process occurs at high intensity, which we attribute to a second mechanism discussed next.

\section{PARAMETRIC INSTABILITIES}

A second mechanism of collective absorption involves parametric instabilities. which are the nonlinear coupling of the light through the ponderomotive force to various plasma modes. ${ }^{11.12}$ In these processes the ponderomotive force couples the harmonic oscillator equations for
Fig. 6. The anharmonic restoring force on oscillating electrons near the density jump. plasma waves with those for light waves at a different frequency from the laser. Typically, the conditions for instability are met most readily when the three natural freql: $n$ ncies, $\omega_{1}, \omega_{0}$, and $\omega$, and the three wave vectors, $k_{1}$. $k_{0}$, and $k$ satisfy conservation of energy and momentum:

$$
\omega_{1}=\omega_{0}-\omega
$$

and

$$
k_{1}=k_{0}-k
$$

where the subscript 0 represents the pump laser. 1 represents the scattered (light) wave, and no subscript represents the plasma wave. The most important modes appear to involve coupling of light waves to electron plasma waves and ion sound waves. The coupling to electron plasma waves. known as stimulated Raman scattering, occurs at low densities, $\omega_{p}^{2}<\omega^{2} / 4$, and excites a broad spectrum of electron plasma waves that accelerate electrons to high energy over a long (typically 

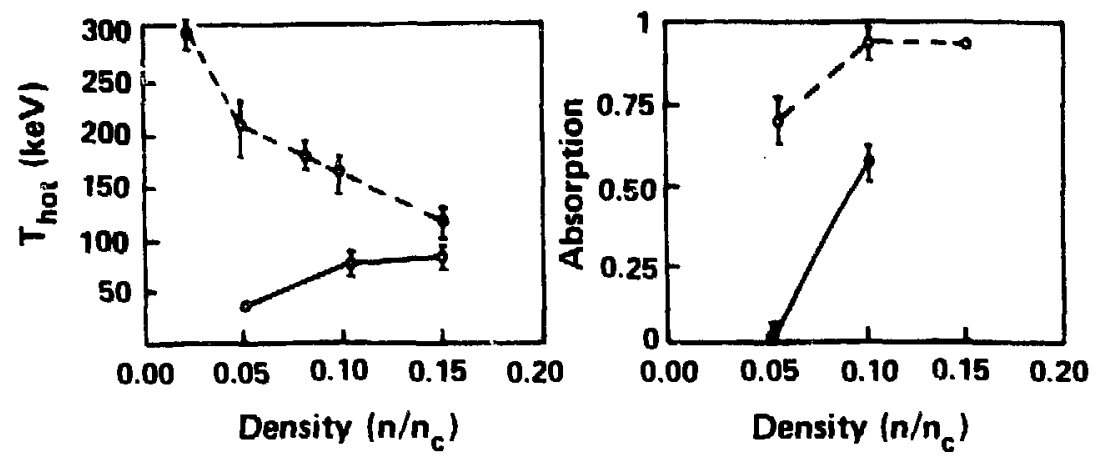

Fig. 7. Hotelectron temperatures and absorption in a long-scale-length plasma from WAVE. The solid curves correspond to a single pass of the laser light through the plastna. Dashed curves correspond to a reflection of the laser light at the plasma boundary and two passes through the plasma.

1-mm) distance. (The production of extremely energetic particles is being harnessed in the beat wave acceleration process. In this process. two lasers with a frequency difference equal to the plasma frequency are used to enhance the plasma wave amplitude over that generated by Raman scattering itself.) This acceleration over relatively large distances contrasts to the resonant absorption process described above. In addition, right at the quarter-critical density, the incident light can directly decay into two plasmons. The nonlinear state of these instabilities depends strongly on the density scale length and temperature of the underdense plasma. Unfortunately, plasma simulations are not able to selfconsistently calculate the large-scale underdense plasma blowoff, and the experiments at $10 \mu \mathrm{m}$ have been unable to measure it. Therefore, only representative calculations can be made in which one estimates the plasma initial conditions. These suggest that, as the plasma scale lengths approach a millimeter, this process may begin to dominate. The hot-electron temperature and absorption scaling from simulations are shown in Fig. 7. The hotelectron temperature is much higher at moderate intensities than it is from resonant absorption.

An additionai coupling path of light to ion sound waves in underdense plasmas (known as stimulated Brillouin scattering) ${ }^{13}$ results in less plasma heating because of the greater mass of the ions, although it has the potential of reducing the absorbed laser intensity. Calculations suggest that the ion waves become nonlinear so rapidly that the light is not scattered away but merely adds to the plasma heating rate. This heating rate, however, is lower than that from electron plasma waves. This process may be much more important at short laser wavelengths, where the ion sound waves do not become as nonlinear.
In addition to backscattering, there are also selffocusing and filamentation instabilities of the incident light. ${ }^{14}$ Ponderomotive force-driven filamentation has a similar gain length to Brillouin scattering when the ions are very warm, $T_{1}=T_{e}$. At low intensities there is a thermal self-focusing instability resulting from bremsstrahlung heating that can reduce the density in a channel, refracting the light witnin the channel, which raises the intensity and further heats the plasma. This latter process may be particularly troublesome at short wavelengths. This instability can be viewed as arising from the strong anisotropy in the pressure of the incident light wave.

A number of parametric instabilities at the critical surface have been identified theoretically. ${ }^{15}$ However, the steep density gradients induced by the ponderomotive force severely reatuce these instabilities.

If fusion with $\mathrm{CO}_{2}$ lasers is to work, one must stay at intensities and plasma conditions that do not allow parametric instabilities to develop and that rely on resonant absorption in a steepened density gradient to maintain the lowest hot-electron temperature. These conditions appear difficult to achieve at best. As mentioned earlier, some additional absorption process appears to be occurring above $10^{13} \mathrm{~W} / \mathrm{cm}^{2}$. Recent simulations at an intensity of $2.5 \times 10^{15} \mathrm{~W} / \mathrm{cm}^{2}$ run for tens of picoseconds instead of only a few picoseconds ${ }^{4}$ (made possible by the substantial increase in size of the Los Alamos computer facility) show a similar phenomenon. Figure 8 shows a contour plot of the density surface late in time. Note that the originally smooth, sharp density gradient has begun to break up and become rough. Associated with this roughness is a substantial increase in the absorption coefficient from about 25 to $60 \%$. At the same time, the 


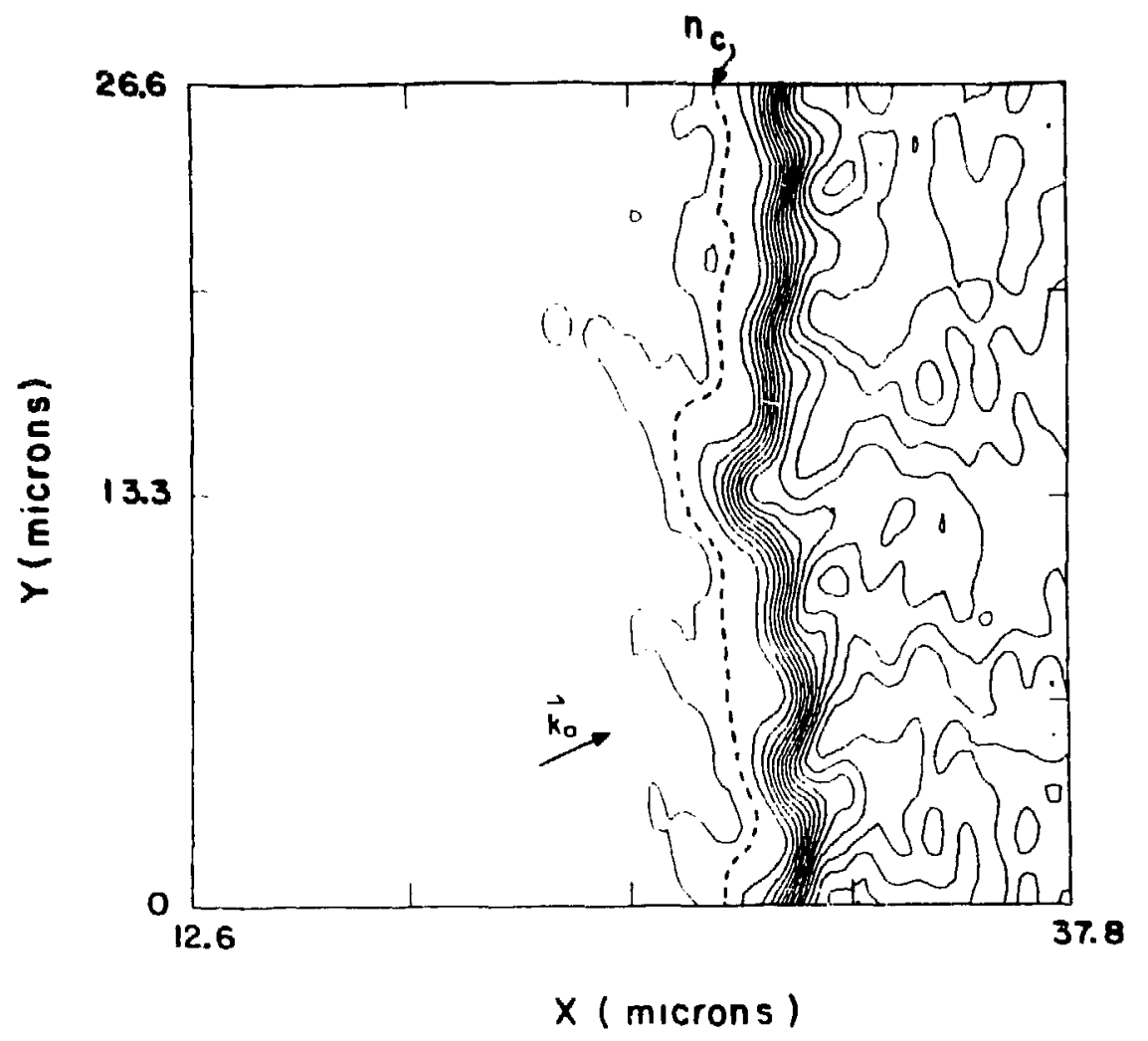

Fig. 8. Contour plot of the density late in time.

hot-electron temperature incieases by a factor of 2 or 3 over that calculated for resonance absorption on the initially smooth surface. At $10^{16} \mathrm{~W} / \mathrm{cm}^{2}$, the surface is observed to become even more turbulent. Basically, the parametric instabilities at the critical density that were suppressed by the sharp gradient appear to become dominant at high intensity. This process may explain the increased hot-electron temperatures (hundreds of kiloelectron volts) observed on Helios at high intensities. ${ }^{1,3}$

We see then that the large amplitude of the laser radiation results in a highly nonequilibrium state of the plasma. It is so far from equilibrium that, for example, classical shock wayes are altered. The region where iesonant absorption occurs corresponds to a phase transition between a hot plasma in the presence of the laser light to a colder plasma without the laser light present. In this region rarefaction shocks ${ }^{16-18}$ are generated, which have very different properties from conventional shock waves. Matter is put into an extremely unusuai state that is probably only reproduced in exotic astrophysical situations. The fact that most of the absorbed laser energy is placed into a few energetic particles significantly reduces the impiosion efficiency of fusion targets and makes the task very difficult.

\section{FAST-ION GENERATION}

Just as there are collective effects that control the absorption of laser light. collective plasma effects can also dominate the transport of electrons into targets. The hot-electron pressure in the corona can collectively accelerate the coronal ions to extremely high energies by collisionless processes. In the simplest model, a small number of electrons leave the target, but the nonzero impedance and inductance of the target support stalk allow electrostatic potentials of hundreds of kilovolts to develop at the target, confining the remainder. The confined electrons accelerate ions by working against the coronal plasma as they try to escape. Experimental measurement of the fast-ion energy shows that a substantial fraction of the absorbed laser light goes into fast ions. 
particularly at high intensity (Fig. 9). For targets that are thick to hot electrons, so that an electron has only a single chance to bounce off the electrostatic sheath surrounding the target and work against the coronal ions, theoretical calculations (ignoring albedo effects) indicate that the fraction of energy in fast ions cannot exceed 5-10\%, which is the fraction of electron energy lest in reflecting from the expanding plasma. Thus, we infer from these data that a mechanism exists to trap the electrons in the corona, allowing them to lose more of their energy to fast ions. This process is generically known as flux-limited transport. That is, there is some process that reduces the mean penetration velocity to less than it would be from a simple diffusion model. One process that can cause this phenomenon involves the generation of intense magnetic fields by the high-energy electrons themselves.

For a laser spot of finite size on a target surface or for not completely uniform illumination of a target. there is a temperature gradient along the surface over the laser spot, a strong density gradient along the surface over the laser spot. and a strong density gradient normal to the surface under the spot. These density and temperature gradients, which are perpendicular to each other, cause 8 magnetic field to be generated by the curl of the ambiopolar electric field:

$\partial \mathrm{B} / \partial \mathrm{t}=-\nabla \times \mathbf{E}=\nabla \mathrm{n} \times \nabla \mathrm{T} / \mathrm{n}$.

The generation rate is extraordinarily high with a field of I MG reached in 1 ps with a density gradient of $10 \mu \mathrm{n}$, a spot size of $100 \mu \mathrm{m}$, and a temperature $\mathrm{T}$ of $50 \mathrm{keV}$. In the time it takes an electron to cross the laser spot, the field is strong enough to reduce the gyroradius of an electron to less than the density scale height. The lectrons can no longer free-stream into the target bu are confined by the magnetic field. In VENUS simulati/ons ${ }^{19}$ the electrons EXB drift along the target surface to zreat distances from the initial laser spot. ${ }^{20}$ This reduces the transport inward under the laser spot and enhancels the electron energy carried far from the laser spot. One of the consequences of the self-generated fields is that meiny of the ions are accelerated in an intense ion jet or plume normal to the target surface.

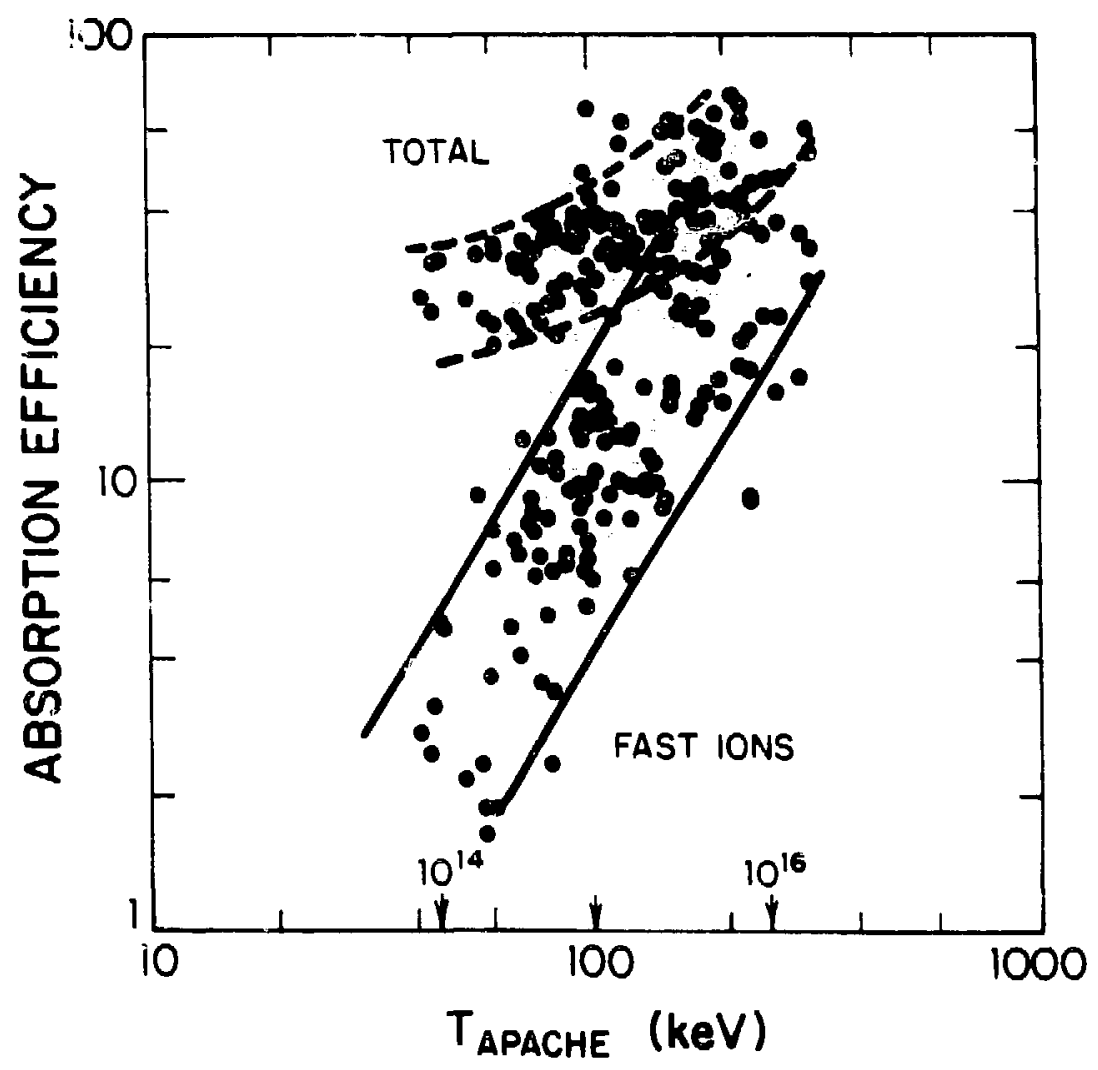

Fig. 9. Fast-ion production efficiency as a function of hot-electron temperature (from the APACHE hard $x$-ray spectrometer) and intensity. 
Figure 10 shows the angular distribution of ions leaving a disk target as measured by calorimeters with three different thresholds provided by filtering. The plume has half-width at half maximum of about $10^{\circ}$, in excellent agreement with particle simulations using VENUS. For targets smaller than about $1 \mathrm{~mm}$, including small spheres, the effect is "washed out," and the ion angular distrubition becomes more isotropic. The effect is also washed out in thin targets, where electrons can travel from the laser spot by reflexing through the shell and disrupting the current flow that creates the magnetic fields. This acceleration process is quite equivalent to the one that occurs in intense ion diode experiments except that it is much higher intensity than any conventional diode. The accelerated ions could be used to drive a fusion target except that the collimation is not of sufficient quality to allow a large separation beween the diode and the ion target to prevent electron preheat. Experiments also suggest that ion emission may remain large at high intensity when the illumination is more uniform. At this point there is no conclusive theory that would reproduce this effect. Measurements of the ion velocity distributions with magnetic analyzer spectrometers [so-called Thomson parabolas, which are ion spectrometers with parallel $\mathbf{E}$ and $B$ fields and which produce velocity spectra $N(v)$ along parabolic tracks in the detector plane with different parabolas for each $\mathrm{Z} / \mathrm{A}$ ] indicate that much of the ion energy is carried by hydrocarbons independent of the target material. These hydrocarbons are surface contaminants on the target that are accelerated to the highest velocities in part because the lowest $Z$ ions are accelerated most rapidly in the complex multispecies expansion. Although the ion spectrim from such a multispecies expansion is remarkably complex ${ }^{21}$ and difficiuli io calculate theoretically, its gross properties are deceptively simple. As the ion mean energy is not far above that required to penetrate a $0.5-\mu \mathrm{m}$ nickel filter, we can determine the ion "spectrum" by a set of transmission measurements in an array of differently filtered calorimeters. Figure 11 shows examples of such ion spectra for high- and low-intensity cases. Not only are these transmission curves well behaved, they can readily

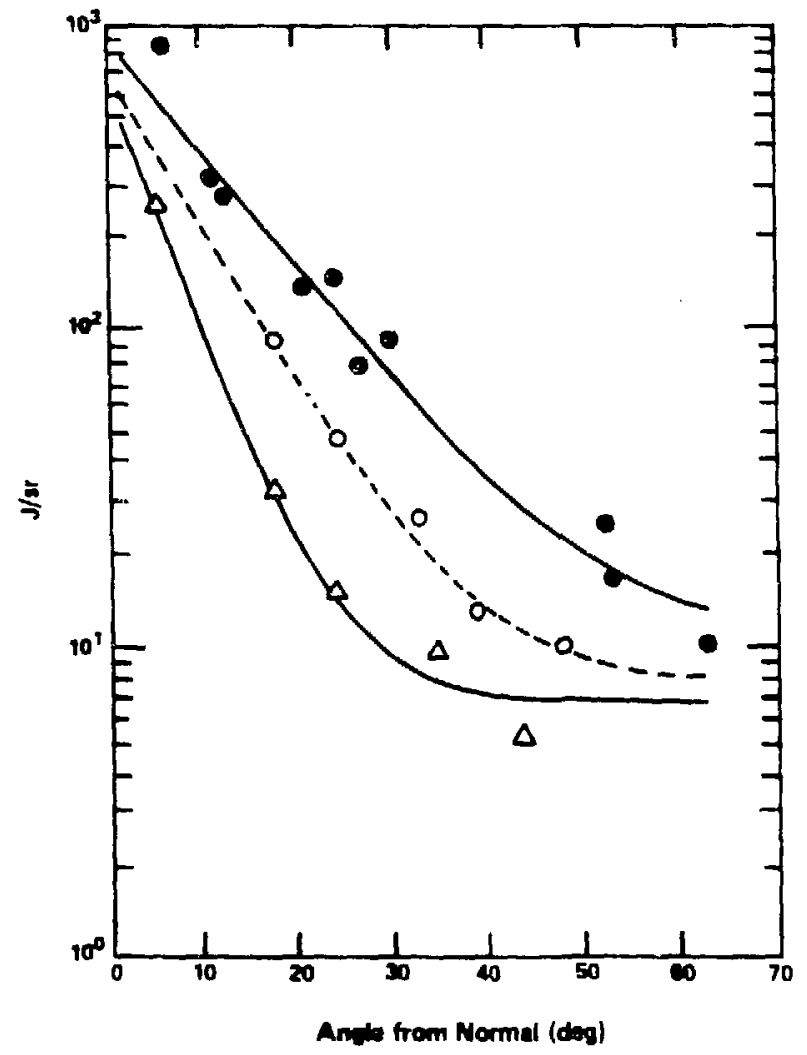

Fig. 10. Fast-ion angular distribution: - all inn energies, $O E \geqslant 100$ $\mathrm{keV} / \mathrm{amu} . \Delta \mathrm{E} \geqslant 500 \mathrm{keV} / \mathrm{amu}$. 


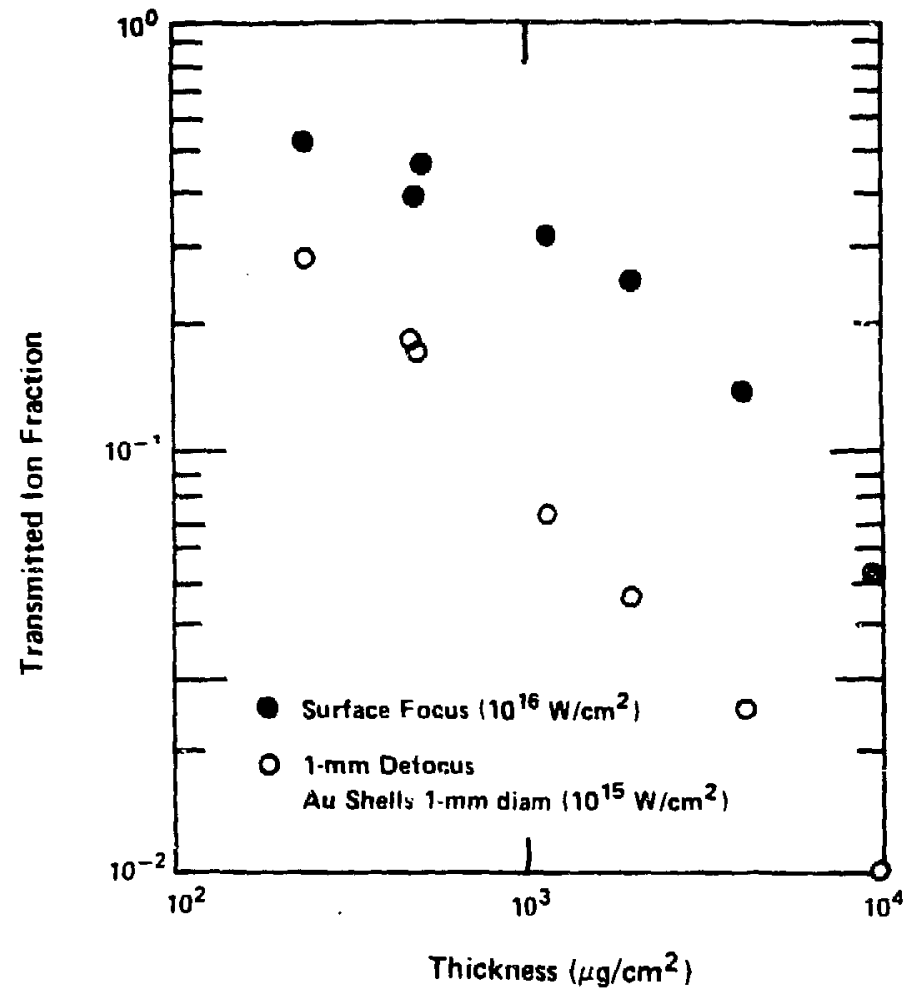

Fig. 11. Ion transmission spectra.

be fit to an isothermal expansion model taking the hotelectron temperature $\mathrm{T}$ directly from the bremsstrahlung measurements if we assume $\mathrm{Z} / \mathrm{A} \approx 1 / 2$. Transmission data as in Fig. 11 are useful in evaluating target design concepts. For example, the mass required to stop one half of the ion energy is an important parameter that can be used to determine whether this ion energy is exploitable in target design.

Whereas ion stopping powers are a weak function of $Z$. very heavy ions (such as tantalum and gold) have an appreciably shorter range than protons or carbon. Based on an analysis of the multispecies ion expansion, we conjectured that if low- $Z$ surface contaminants could be removed, atoms of high- $Z$ target material could be accelerated to similar velocities (the fastest proton in a typical expansion, measured with time-of-flight scintillator detectors. typically has a velocity of about $2 \times 10^{9}$ $\mathrm{cm} / \mathrm{s}$ ). In an experiment in which tantalum targets were heated with electron bombardment to white heat to drive off hydrocarbon, we observed energetic tantalum ions. The fastest ion observed corresponded to an energy of $500 \mathrm{MeV}$ for tantalum, and Thomson spectrometer data confirmed the absence of protons in the expansion while indicating that the tantalum ions were as much as 60 times ionized.

For targets with thickness less than the hot-electron range, electrons can pass through the target many times. reflecting off the expanding plasma sheath each time. This can greatly increase the absorbed energy placed into fast ions.

\section{ASTROPHYSICAL IMPLICATIONS}

Despite that fact that conditions produced in a target by a $\mathrm{CO}_{2}$ laser are not favorable for producing fusion, they result in some of the highest energy density plasmas yet produced in the laboratory. For example, the hotelectron source produced by the laser is equivalent to a high-current, low-impedance electron beam source with a current in excess of $10^{8} \mathrm{~A}$ and voltages of hundreds of kiloelectron volts. This results in a souice impedance of about $0.001 \Omega$, much lower than can be produced by any conventional pulsed-power source. At the same time, this 
intense "thermal" electron beam produces a reasonably collimated jet of space-charge-neutralized plasma traveling at speeds of $10^{8}-10^{9} \mathrm{~cm} / \mathrm{s}$ and maintaining its collimation over distances of at least $4 \mathrm{~m}$. Although the distance scales and time scales are perhaps 25 orders of magnitude smaller, these jets are reminiscent of the galactic jets that have been so clearly identified from high-resolution radio wave photographs. This similarity extends even to the microwave emission of these objects. as we will discuss shortly. We argue here that the ability to measure not only the optical emission of these objects but also the ion motion directly makes study of these phenomena particularly attractive in the laboratory and highly complementary of the elegant astrophysical measurements. The intense magnetic fields that are in timately related to the generation of these ion jets are also known to be important in the accretion of matter in white dwarfs. Although we cannot. of course, study this accretion process directly, the detailed study of the mechanisms of acceleration and the verification of theoretical models could have a profound impact on astrophysical models.

An important means of knowing of the existence of galactic jets comes from their radio emissions. However. there remains considerable debate as to the processes that dominate in the production of microwates from these extended ubjects. Similarly. in experiments done on Helios and Antares, we are able to observe these plasmas in the microwave region of the electromagnetic spectrum. Quite remarkably, these plasmas appear to be incredibly bright in the radio spectrum. In the range of $0.1-5 \mathrm{GHz}$. over a gigawatt of power is observed. Likewise. in measurements of emission in the 26- to $40-\mathrm{GHz}$ band. nearly I GW has been observed. This is $0.01-0.1 \%$ of the incident laser light converted to microwaves. At the same time. the source is nearly a point source and has a pulse shape that nearly follows the laser pulse shape. with a rise time of 0.2 ns. The surprising efficiency of emission may be coupled to an enhanced level of plasma waves in the expanding corona. which couple efficiently to microwave emission. This is one of the important candidates for emission from galactic jets as well as from solar flares. A more detailed investi-ition of these processes in the laboratory could allow a significant discrimination beiween various theories of emission (such as synchrotron radiation or plasma wave emission) in astruphysical plasmas. Besides just measuring properties of the unique plasma produced by intense $\mathrm{CO}_{2}$ laser light. the oppurtunity also exists for studying the propagation of the plasma plume through other ambient plasmas that could be imposed in the target chamber. This could shed light on the propagation of energetic ion jets through ionized plasmas. Do they maintain their physical integrity as they expand through the plasma? Is microwave emission altered as they strike another plasma? What is the emission in the submillimeter part of the spectrum? Theory suggests that it is much higher than at longer wavelengths. If one compares a normalized spectrum from a typical Antares target with that of the Crab nebula. for example. there is a striking similarity. Is this a coincidence or is there something in common between some of the emission processes? The uncovering of a wealth of exotic plasma behavior at extremely high laser intensity raises the possibility of new and important experiments that could not have been imagined a decade ago. The understanding of such processes already obtained from our theoretical and experimental efforts may provide insights valuable to our understanding of astrophysical processes.

\section{REFERENCES}

1. A. Hauer. et al. "Suprathermal Electron Generation. Transport. and Deposition in $\mathrm{CO}_{2}$ Laser Irradiated Targets," in Laser Interaction and Related Plasma Phenomena, V. 6. p. 479 (Hora and Miley, ids., Plenum, 1984).

2. J. U. Brackbill and D. W. Forslund, J. Comp. Phys. 46. 271 (1982).

3. W. Priedhorsky. D. Lier, R. Day, and D. Gerke. Phys. Rev. Lett. 47, 1661 (1981).

4. D. R. Bach, D. E. Casperson, D. W. Forslund, S. J. Gitomer. P. D. Goldstone. A. Hauer, et al., Phys. Rev. Lett. 50, 2082 (1983).

5. D. W. Forslund. J. M. Kindel, K. Lee, E. L. Lindman, and R. L. Morse. Phis. Rev. A 11, 679 (1975).

6. D. W. Forslund. J. M. Kindel. K. Lee, and E. L. Lindman. Phys. Rev. Lett. 34, 193 (1975).

7. R. L.Carman. D. W. Forslund, and J. M. Kindel, Phys. Rel' Lett. 46. 29 (1981). 
8. D. W. Forslund, J. M. Kindel, and K. Lee, Phys. Rev. Lett. 39, 284 (1977).

9. B. Bezzerides, S. J. Gitomer, and D. W; Forslund, Phys. Rev. Lett. 44, 462 (1980).

10. B. Bezzerides, R. D. Jones, and D. W. Forslund, Phys. Rev. Lett. 49, 202 (1982).

11. D. W. Forslund, J. M. Kindel, and E. L. Lindman, Phys. Fluids 18, 1002 (1975).

12. D. W. Forslund. J. M. Kindel. and E. L. Lindman, Phys. Fluids 18, 1017 (1975).

13. D. W. Forslund, J. M. Kindel, and E. L. Lindman. Phys. Rev. Lett. 30, 739 (1973).

14. D. W. Forslund, et al., Phys. Rev. Lett. (1985).

15. D. W. Forslund. J. M. Kindel, Kenneth Lee, and E. L. Lindman. Phys. Rev. Lett. 34, 193 (1975).
16. D. W. Forslund, J. M. Kindel, Kenneth Lee, and E. L. Lindman, Phys. Rev. Lett. 36, 35 (1976).

17. K. Lee, D. W. Forslund, J. M. Kindel, and E. L. Lindman, Phys. Fluids 20, 5 I (1977).

18. B. Bezzerides, D. W. Forslund, and E. L. Lindman, Phys. Fluids 21, 2179 (1978).

19. D. W. Forslund and J. U Brackbill, Phys. Rev. Lett. 48, 1614 (1982).

20. M. A. Yates, D. B. VanHulsteyn, H. Rutkowski, G. A. Kyrala, J. Brackbill, et al., Phys. Rev. Lett. 49 , 1702 (1982).

21. F. Begay and D. W. Forslund. Phys. Fluids 25, 1675 (1984). 


\title{
The KrF Laser: \\ The Advance Toward Shorter Wavelengths
}

\author{
by Reed J. Jensen
}

I f energy is added to water in a swimming pool by using wavelengths comparable to the dimensions of the pool, water quickly peaks and sloshes over the edge. If, however, shorter wavelengths are used, the water, though rippling with motion, remains in the pool. There is an analogous problem when one uses laser energy to drive the implosion of a small fusion pellet. If longer wavelengths, say in the infrared, are used, then a portion of the energy "sloshes over" into undesirable modes (such as a few very hot electrons) that dissipate rather than drive the implosion. As our understanding of the physics of laser fusion has increased, awareness of the importance of fusion drivers with shorter wavelengths has likewise increarad.

However. buildin emits short-wavelength photons is a difficult balancing act for a number of reasons. The balancing becomes obvious when we look at the expression for laser gain. In a simple two-level laser, the gain coefficient (g) obeys the relationship

$$
\mathrm{g} \propto \lambda^{2} / \mathrm{t}_{\text {spont }},
$$

in which $t_{\text {spont }}$ is the lifetime of the excited state against spontaneous emission and $\lambda$ is the wavelength of the emitted photon. The gain itself is proportional to the factor $e^{\text {s. }}$.

As we go to the high excitation levels needed for shorter wavelengths, tne lifetimes of excited states tend, in general, to become short. This means the gain coefficient increases-an apparent advantage (although transition strengths can reverse this trend). But a short lifetime also means the excited atoms spontaneously emit their energy quickly. This latter fact is a disadvantage because the emitted photons stimulate further emissions, resulting in a phenomenon called amplified spontaneous emission (ASE). In large-volume systems, such emission is parasitic, draining energy away too quickly and reducing system efficiency. In other words, it becomes difficult to store large amounts of energy in the lasing medium.

If this trend is resisted by the use of a system that emits short-wavelength photons from an excited state with a moderate lifetime, then the gain coefficient drops because of the factor $\lambda^{2}$. For example, a system with about the same upper-level lifetime as that of the $\mathrm{CO}_{2}$ laser but emitting at a wavelength of 0.1 micrometer $(\mu \mathrm{m})$ rather than $10 \mu \mathrm{m}$ will have an inherent decrease in the gain coefficient of a factor of 10,000 . This would make the gain, proportional to $e^{8}$, negligible. However, UV system ${ }^{\circ}$ almost always have a much shorter lifetime that can result in a high gain.

There are exceptions to these trends that provide promising laser systems in the mid-ultraviolet, but in this region technical difficulties with optics and windows add to the difficulty of the balancing act. For example, common optical materials absorb strongly at these wavelengths. In essence, conventionai optics will not suffice for wavelengths shorter than about $\frac{1}{j} \mu \mathrm{m}$, and truly novel techniques must be used.

\section{THE KRYPTON FLUORIDE LASER}

We are currently developing a: ios Alamos the krypton fluoride $(\mathrm{KrF})$ laser-a system that balances these facets to yield a highly efficient laser able to emit intense bursts of short-wavelength photons. For example, the $\mathrm{KrF}$ laser operates at $\frac{1}{2} \mu \mathrm{m}$, close to the shortwavelength limit for optics but, fortunately, just on the conventional-optics side. The excited-state lifetime of the 
system is short-due both to spontaneous emission and to deactivetion from collisions-making it impossible to store significant energy in the lasing medium. Counterbalancing this disadvantage is the laser's high gain, which yields a system able to amplify efficiently a rapid series of short pulses As a result, considerable energy can be stored outside the laser during the beam's flight to the target. Such storage is accomplished by using a novel multiplexing scheme in which time-of-flight differences cause the series of pulses to meat simultaneously at the target. Thus we are developing a system that will be able to generate short, intense pulses of $\frac{1}{4}-\mu \mathrm{m}$ light highly desirable for the study of the physics of laser fusion at short wavelengths.

\section{An Overview of the Laser}

In early 1975 J. E. Velazco and D. W. Setzer at Kansas State University suggested using krypton and fluorine gas as a lasing medium. ${ }^{1}$ and in June $1975 \mathrm{~J}$. J. Ewing and C. A. Brau at AVCO Everett Research Laboratory reported the first laser oscillation for the highly efficient KrF system. ${ }^{2}$ The potential energy curves shown in Fig. 1 illustrate the major reason for the high efficiency. The ground state is repulsive, and the "molecule" readily dissociates into neutral krypton and fluorine atoms. As a result, there is no accumulation of molecules in the lower laser level, and a significant population inversion can be achieved. The main laser transition nccurs between the $B^{2} \Sigma \frac{1}{2}$ excited state, made up of an attractive $\mathrm{Kr}^{+} \mathrm{F}^{-}$ion pair. and the $\mathrm{X}^{2} \Sigma_{\frac{1}{2}}$ ground state. This transition can be pictured as the ion pair reverting back to the dissociating molecule by transferral of an electron from $\mathrm{F}^{-}$to $\mathrm{Kr}^{+}$and emission of a photon with a wavelength of $\frac{1}{4} \mu \mathrm{m}$.

Gain. Measured gain coefficients for the KrF laser are in excess of 10 per cent per centimeter, so that a 1 -meter amplifier would have a gain of $e^{10}$, or about 20,000 , per pass through the amplifier. As pointed out above, such huge gains will not allow storage of large amounts of energy in the lasing medium as a population inversion; the excessive gains cause a loss of upper-level population by amplified spontaneous emission. By using the multiplexing scheme, however, we are able to extract the energy as a series of short pulses whlle the laser is being pumped. This extraction suppresses the gain by reducin the gain coefficient according to the equation

$$
g=\frac{B_{0}}{1+\frac{I}{I_{\text {eat }}}},
$$

where $\mathrm{B}_{0}$ is the gain coefficient at low intensities (tht small-signal gain), I is the laser intensity in the medium and $\mathrm{I}_{\mathrm{sut}}$ is the so-called saturation intensity (an intensit) based on photon energy, the stimulated emission cros: section, and the upper-state lifetime of the lasing me dium).

It is apparent that if energy is extracted from the amplifiers while the laser is running at an intensity thret times $I_{\text {ant }}$, there will be a fourfold decrease in the actua gain coefficient. This reduction will bring the system gair down from about twenty thousand to a few hundred.

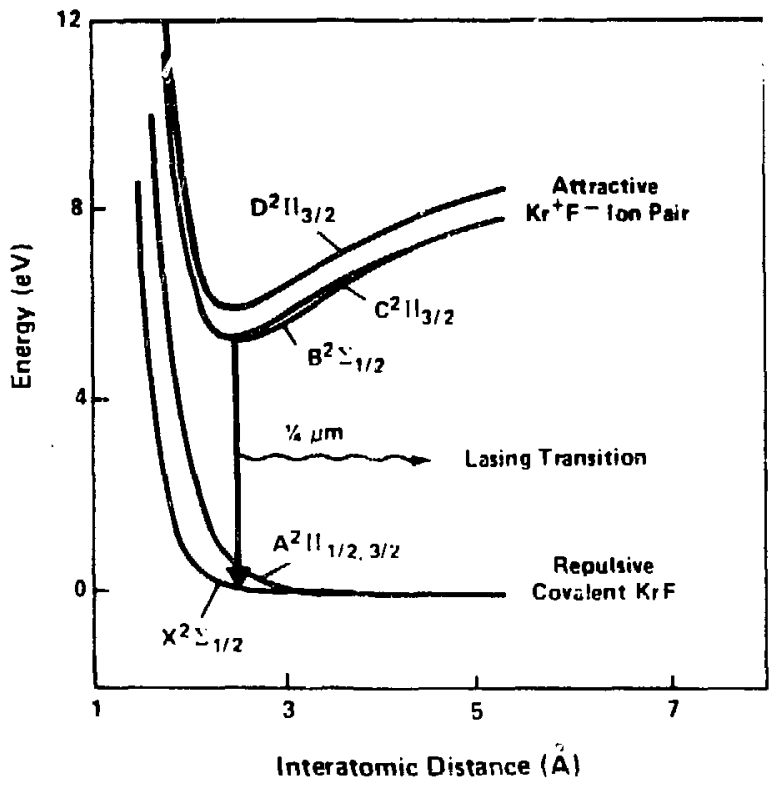

Fig. I. The potential energy diagram for the KrF laser. The upper state of the lasing transition $\left(B^{2} \Sigma\right)$ is one of the states for the $\mathrm{Kr}^{+} \mathrm{F}^{-}$ion pair. The lower state $\left(\mathrm{X}^{2} \Sigma+\mathrm{u}\right)$ is one of two covalent states that are repulsive and so dissociate. eliminating $\mathrm{KrF}$ molecules from the lower level and ensuring a large population inversion. [(Adapted from P. J. Hay and T. H. Durning, Jr., Journal of Chemical Physics 66, 1306 (1977)|. 


\section{Gas Kinetics}

In a practical sense, the efficiency possible in the system is determined by how atoms are pumped into the upper laser level. Typically, the energy is added by ionizing a gaseous mixture that includes argon. The argon atoms play an active role by forming intermediate syjecies, as shown in Fig. 2, a simplified flow chart for the gas kinetics of the Ar-Kr-F ${ }_{2}$ system. Initially, all three types of atoms are ionized (here using an electron bearn), but the path (1) that froduces the largest population of $\mathrm{KrF}$ ion pairs $\left(\mathrm{KrF}^{*}\right)$ in the upper laser level involves $\mathrm{Ar}_{2^{*}}^{+}$. The second most important path (2) involves the $A r F$ ion pair ( $\left.A r F^{*}\right)$. Once the $\mathrm{KrF}$ ion pair is reached, the desired exit channel is, of course, dissociation to $\mathbf{K r}$ and $F$ and emission of a photon.
The efficiency for conversion of pump energy to upperstate population could conceivably be increased by using more direct or lower energy channels to the KrF ion pair. In fact, immediate gains in efficiency can be realized by increasing the amount of krypton in the mixture, although this gas is more costly. Such an approach would emphasize the path (3) in which krypton is ionized directly-a more efficient route because of krypton's lower ionization energy.

\section{Undesirable Absorption}

Many of the species pictured in Fig. 2 absorb light of KrF frequency. What constraint does this place on the design of the KrF laser? Generally, for any absorbing

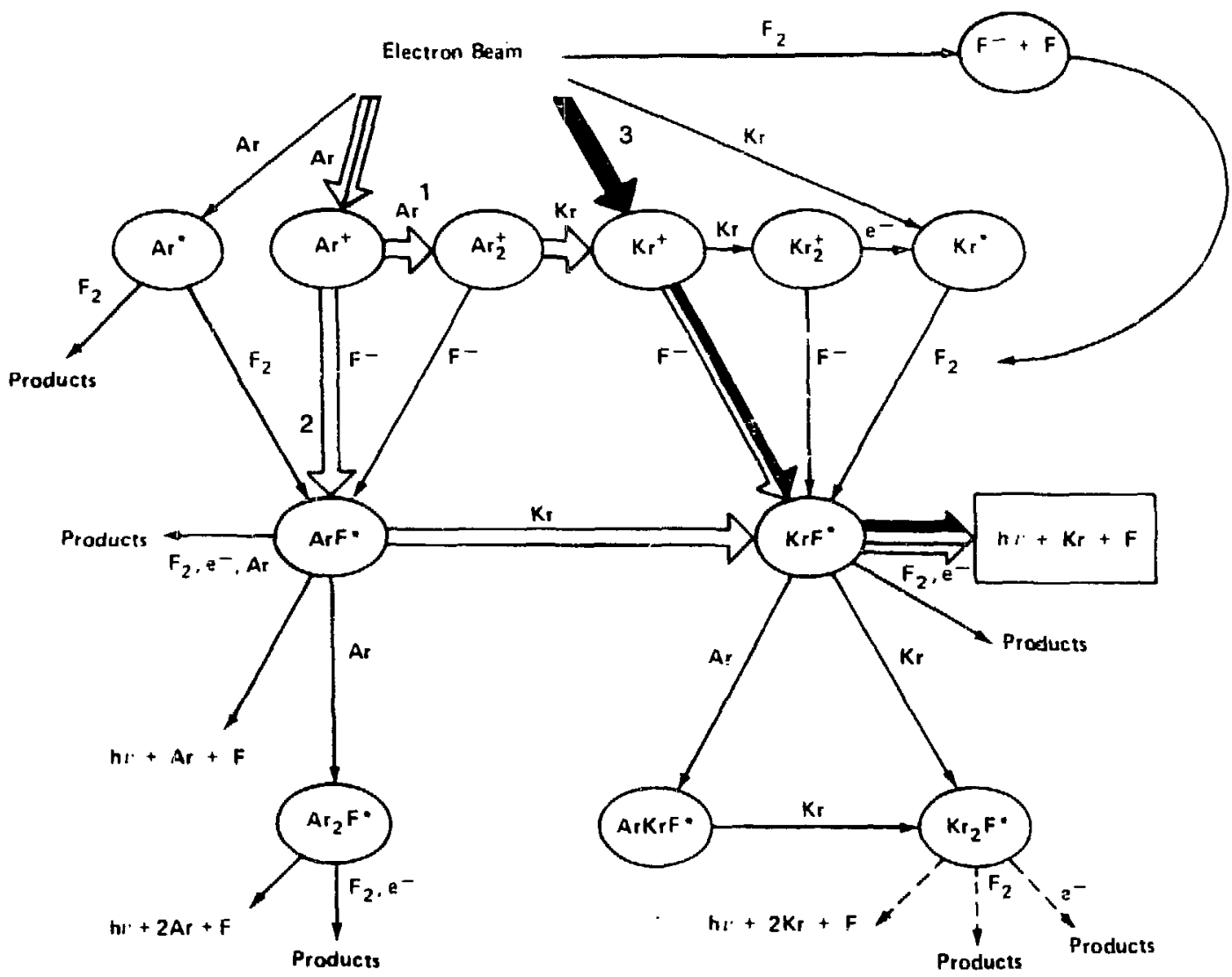

Fig. 2. Kinetic partways for the $\mathrm{KrF}$ laser. Initially, all three atoms in the Ar- $\mathrm{Kr}-\mathrm{F}_{2}$ gas mix are ionized, but the most important path (1) to the $\mathrm{KrF}$ ion pair ( $\mathrm{KrF}^{*}$ )passes through $\mathrm{Ar}^{+}$and $\mathrm{Ar}^{+}$intermediates before a krypton atom is ionized. The $\mathrm{Kr}^{+}$ion then combines with a previously ionized fluorine atom. The second most important path (2) also starts with $\mathrm{Ar}^{+}$but then forms on $\mathrm{ArF}$ ion pair (ArF*) before exchanging with a krypton atom. Path (3) is energetically favorable because krypton ionizes easily, but the path only becomes important as the amount of krypton gas in the mix is increased. 
species there is an intensity at which the absorption saturates-that is, the light overpowers the absorption process and starts to pass freely through tine system. The uence for this condition is given by

$\phi_{\mathrm{at}}=\frac{1}{\tau \sigma}$,

where $\tau$ is the relaxation time and $a$ is the absorption cross section for the absorbing species.

Unfortunately, some of the absorbing species in the $\mathrm{KrF}$ laser gas have low values of to and do not saturate at fiuences used for the design of this laser. The system, therefore, has a practical upper iimit on the growth of fluence in the lasing medium. In other words, we have another balancing task; as intensity or fluence increases, the gain saturates but absorption by other species does not. It is believed that the limiting fluence for the $\mathrm{KrF}$ system is about 10 to 100 megawatts per square centimeter. Greater intensities can only be reached by using optical focusing and other techniques that are applied beyond the last amplifier.

\section{Electron Beam}

Ionization of the laser gas mix also has its difficulties. The gas contains fluorine, a halogen, which is an efficient electron scavenger. In fact, the formation of $\mathrm{F}^{-}$by electron attachment plays a key role in the pumping scheme of Fig. 2. However, standard gas-discharge techniques used with many gas iasers generate electrons with energies of a few tenths of an electron volt (eV). Because attachment energies are also of this order, such a discharge has trouble sustaining itself if electron scavengers are present.

Further, in a standard ionization-stabilized discharge system, there is a tendency for the discharge to constrict into a few very hot arcs. This phenomenon leaves most of the gas unpumped, whereas the gas in the vicinity of the arcs is far overpumped and overheated. Even though a preionization device may help generate more homogeneous current flow, the discharge still tends to constrict into hot arcs because of the temperature dependence of the coefficient $\beta$ in the electron replenishment equation,

$\mathrm{e}^{-}+\mathbf{M} \rightarrow \mathrm{M}^{+}+\beta \mathrm{e}^{-}$

In other words, the number of secondary electrons generated when a primary electron collides with atoms in the medium increases rapidly with temperature, and any area of enhanced conduction quickly forms an arc.

The solution to these problems, which would be especiaily severe at the higher intensities required in amplifiers, is to ionize the ges directly by pumping with an electron beam. In this case, the electrons enter the laser volume with energies of several hundred kiloelectron volts, and any difficulties with electron attachment are overpowered.

Likewise, an electron bsan device avoids runaway arcs by injecting electrons directly into the gas through thin foil windows (Fig. 3). Electron homogeneity is established $b_{j}$ field emission and propagation physics in the vacuum region around the cathode. The resulting even distribution of the primary electron current from the electron beam dominates all physics in the laser volume. Thus, the primary current in any particular volume of gas does not depend upon the conductivity of that gas.

Typically, electrons enter the laser volume at energies of 400 to $800 \mathrm{keV}$. In primary collisions gas ionization generates secondary electrons with an energy loss to the primary electron of about $30 \mathrm{eV}$ per ionization. Thus each primary electron requires thousandis of collisions to deposit its energy in the gas. Bcth primury and secondary electrons participate in the various pumping and attachment processes shown in Fig. 2.

With this pumping technique we find that for $\mathrm{Ar}-\mathrm{Kr}-\mathrm{F}_{2}$ mixes about 24 per cent of the energy of the beam appears as population of the upper laser level. Once again, we see that the KrF laser has high intrinsic efficiency. The exact value of the efficiency depends, of course, on the conditions of the gas and the pumping circuit (for example, use of more krypton in the gas mixture should increase this value). So far, an overall efficiency from wall plug to laser bean of 4 per cent has been achieved. Such efficiency is still a little less than is achieved for the $\mathrm{CO}_{2}$ laser, but by using improved gas mixtures and electrical delivery systems, we should achieve efficiencies greater than 10\%.

\section{High Repetition}

Because $K_{r} F$ is a gas laser with relatively high efficiency, it can be pulsed at high repetition frequencies. To prepare for a subsequent pulse, the gas needs time for all intermediate fluorides to revert back to the elements and for electron beam-induced shock waves to damp out. This is achieved by exchanging the gas between pulses 


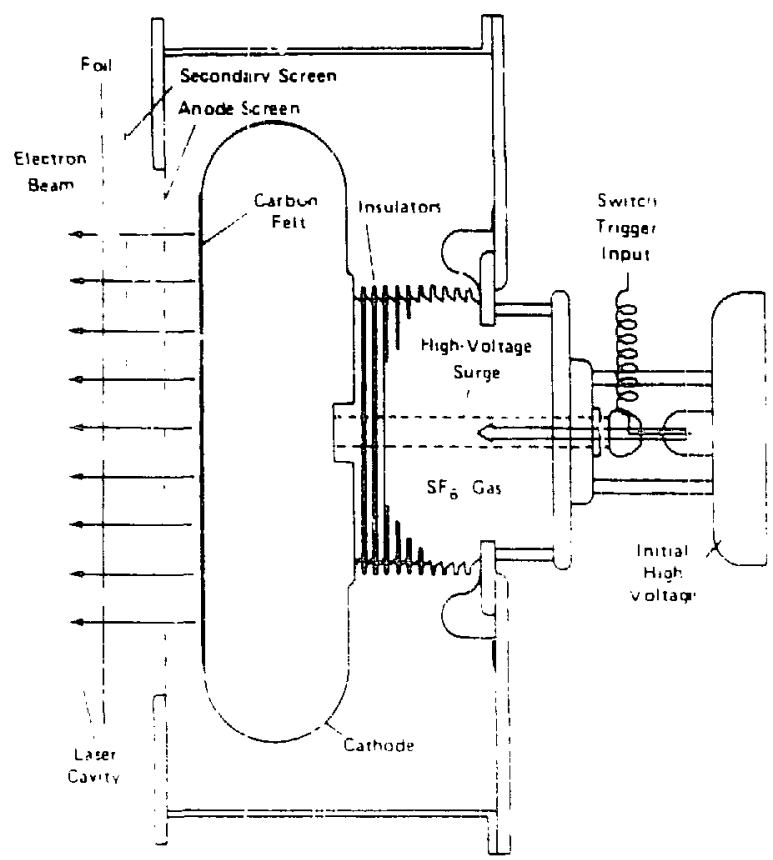

using modestly sized pumps. Also, spurious impurities, formed by fluorine attack on surrounding materials, are removed during the exchange with filters and "getters" I metals. sich as sodicm or calcium that react with the Impurities). (jsing thesiz techniques. KrF lasers have been operated at repatition frequencies greater than a kilohertz.

\section{THE LOS ALAMGS PROGRAM}

Los Alamos has beer heavily involved in the developrent and use of $\mathrm{KrF}$ and other rare-gas-halide lasers since 1976. The work was done originally for the laser isotope separation program, and. in the first year, $\mathrm{KrF}$ l.asers were used to generate macroscopic sampies of Fhotolytic UF 5 from $U_{6}$. At that point we were developing high beam quality. multijoule gas-discharge (rather tnan electron-biam) lasers. In the late 1970s. Los Alamos fioneered gas cleanup schemes that paved the way to the long-lived rare-gas-halide lasers. These efforts culmunated in a $\mathrm{KrF}$ laser that rail at 1 joule per pulse and 500 puises per second. W: also developed. jointly with Rockerdyne. another $\mathrm{Kr} F$ laser that doubles this repetition rate.

At present we are constructing a protctype $\mathrm{KrF}$ laser

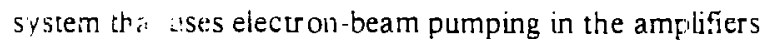
and that will demonstrate the production and extraction of laser energy and the optical techniques needer for a
Fig. il. A scehmatic of the diode for generating the electron be:tm. When the switch trigger is attivated. high voltage surges from righ to left in the cathode. where sectrons are ejected toward the anodt screen. These electrons also flow through a screen tha: eliminates siray secondarv electrons and then through 2 -mil titanium foil into the volume of the laser cavity. The electrons are emitted at energies of several hundred kiloelection volts, and about 95 per cent die trans. mitted througn the foil where they uniformly excite the laser gas. Critical to the design is the cylindrical high-voltage bushinis (insulators and $\mathrm{SF}_{n}$, that is between the switch and the cathode and which prevents undesirable arcing back toward the switch. To give an iden of the size of these electron-beam devices. the emitung surface of onc of the cathodes in our recent large KrF amplifier (the Aurora systs'm) measures 1 by 2 meters

$\mathrm{KrF}$ fusion laser. The system is called Auro*a, and its various components-from the $\mathrm{KrF}$ oscillator through four stages of amplification-are shou'n sctematically in Figs. 4 and 5. The gas-discharge oscillator will emit a single beam consisting of a 5 -nanosecond. $\frac{1}{2}$-joule pulse. To achieve optical multiplexing. the or iginal beam will be split into 96 beams before being se $1 t$ through the various stages of amplification. The final amplifier. salled the Large Aperture Modile ( LAM). will have a lasing volume that is 1 meter by 1 meter by ? meters long and an output totaling about 15 kilojoules ir. the 96 beams.

\section{Multiplexing}

A prime motive for optical multiplexing is cost. It has been estimated that a cost minimum can be achieved for $\mathrm{KrF}$ laser fusion if there are from 50 to 100 beams, each less than 5 nanoseconds in duration, derived from a single elecirical pulse. Fiather than build 100 systems, we intend to use a single opically multiplexed system-a key concept to be demonstrated by the Aurora laser.

Mulciplexing starts with the time and angle encoders (Fig. 5), which will take the original 5-nanosecond plise and split it nto 96 angularly separated beams. Each beam travels a different distance so that each is dela: : $r$ 


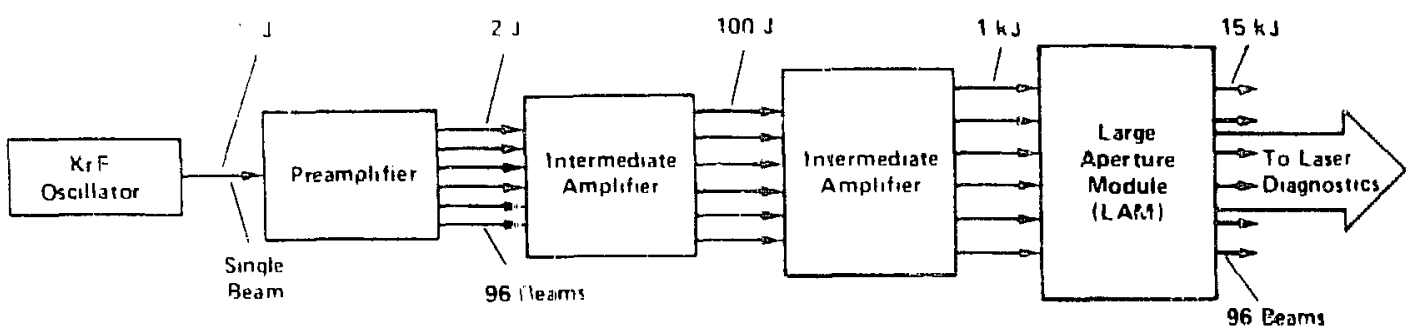

Fig. 4. The power amplifier chain for the aurora KrF laser system starts with a single !-joule beam and ends with about 15 kilojoules divided among 96 beams.

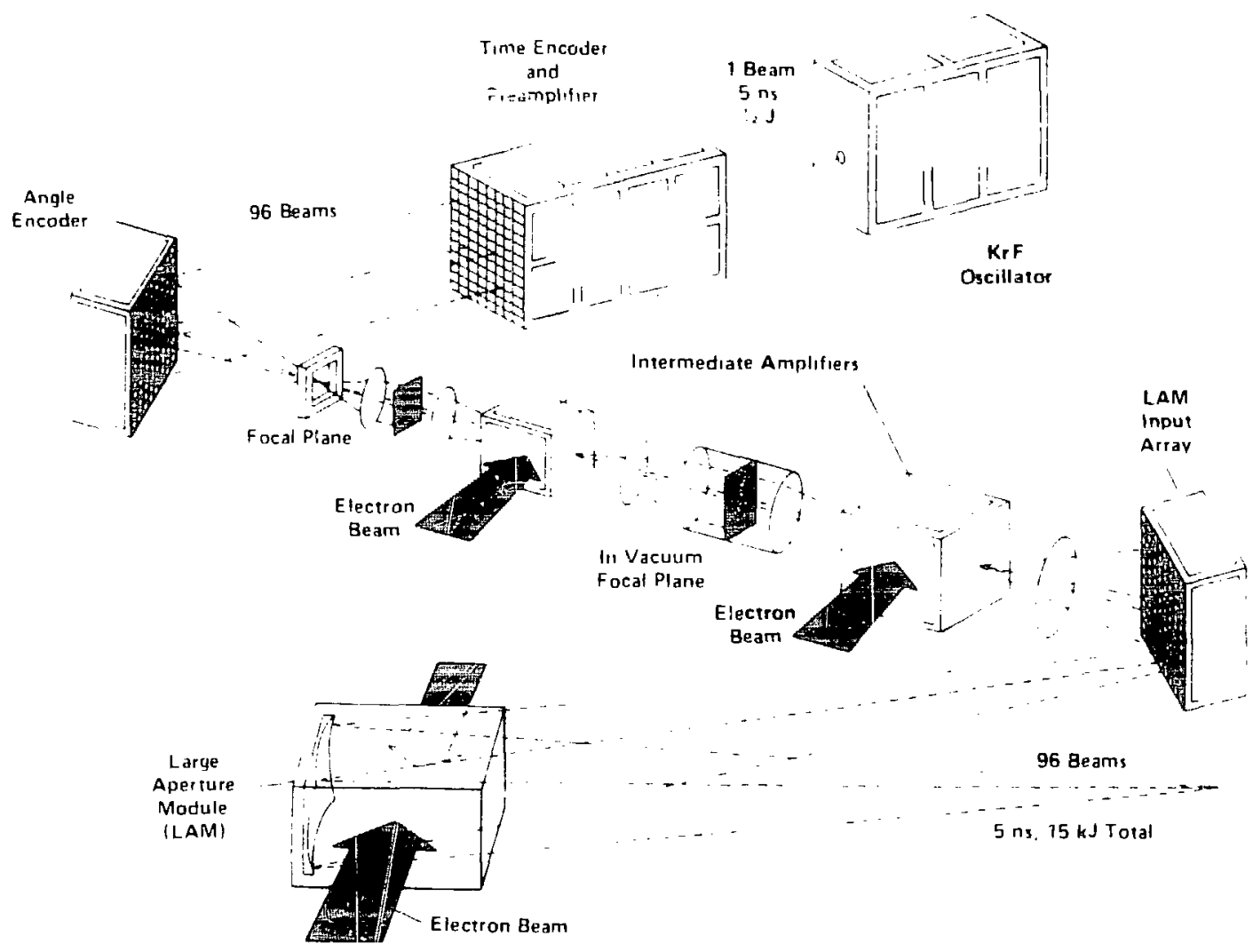

Fig 5. A ronceptual layout for the Aurora laser system. The single beam with 5 -nanosecond pulsewidth from the KrF oscillator is divioed by the time encoder into a traun of 96 temporally separated beams. This splitting is accomplıshed with aperture dividing. the use of partially reflective mirrors called beam splitters, and different path lengths for each beam. Preamplification also takes place in the same apparatus. The angle encuder aims each beam so that it will pass through the central regiun of both intermediate amplifiers. Final amplification to 1s kilojoules takes place in the large aperture module. The oscillator is driven by gas-discharge techniques. but the higher intensitie: in the amplifiers require pumping; by electron beam. A cemultiplexing arrangement is needed after the LAM if the 96 beams are to be brought to the target simultaneously (see Fig. 6). 
differently: the resulting output consists of a train of 5 nar... cond pulses. Because the pulses are angularly separated, each passes through the amplifiers from a sligh.ly different direction. Tine amplifiers are pumped for a relatıvely long time-aboat 600 nanoseconds-while the train of short pulses traverses their volume.

The same time-delay concept can be applied in reverse after the final amplification to cause ail the beams to arrive at the arget at once. A simplified version of this part of the system is snown in Fig. 6, illustrating how the time-of flight for each beam would allow that all beams beams meet cinultaneously at the target.

As noted earlier, the mult plexing technique allows us to take advantage of the higl gain and high efficiency of the $\mathrm{KrF}$ gas laser to generate a short, high-energy pulse on target. The energy of the electron beam discharge is stored in the variously delayed flights of the 96 beams. Mozever, low-cost laser ene:gy is provided by using one system in which the amplifitss run for a relatively long time rather than by using many short-pulse systems.

Figure 7 is a photograph of the final amplifier under construction. The large oval-shaped features are magnets that provide a 3 -kilogauss guide field to direct the electron beam straight into the laser chamber. The two large cylindrical tubes are water dielectric transmission lines that transmit the 1.3 -megavolt electrical pulse to the cathode. One of those cathodes is pictured in Fig. 8 lying on a workbench with the carbon felt emitter surface upright. Figure 9 shows the amplifier being discharged to produce ultraviolet power.

The Aurora laser system will provide experience in nearly all of the issues involved in building a very iarge $\mathrm{KrF}$ laser fusion driver. We will gain experience not only in large $\mathrm{KrF}$ amplifier construction and operation but also in running a whole series of amplifiers with final flux close to the limiting flux for the system. A major issue in this, or any, large $\mathrm{KrF}$ laser system is damage to the windows and mirrors. We must develop coatings with good refiective or transmissive properties that also are resistant to fluorine attack and optical damage. At present, the size of apertures-and, therefore, the overall system cost-depends very sensitively on the threshold for optical damage. Work being performed at Los Alamos and by optical component suppliers is providing greatly improved damage performance.

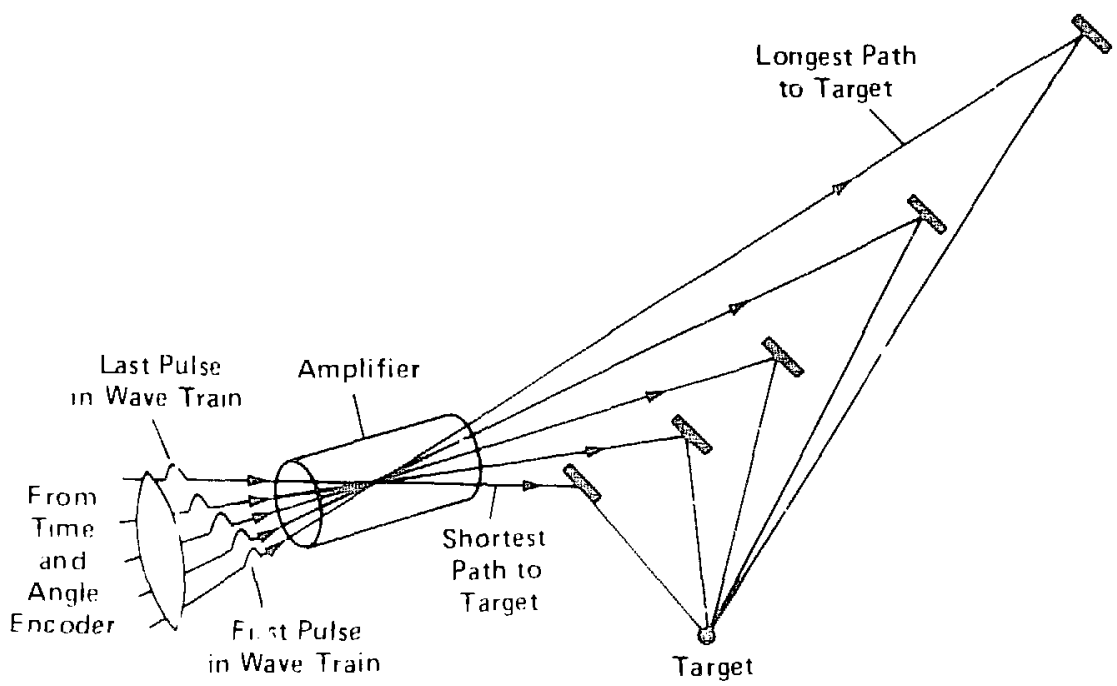

Fig. 6. A smp ified uptical angular multiplexing device. The five beams from the decoder represent a irain of pulses that are separated in time. By adjusting path lengths so that the earliest pulse fcrossing from bottom lelt to upper right) has the longest time-of-flight and the last pulse (crossing horizontally) has the smalles: time of flight, the pulses can be brought together at the target simultaneously. 


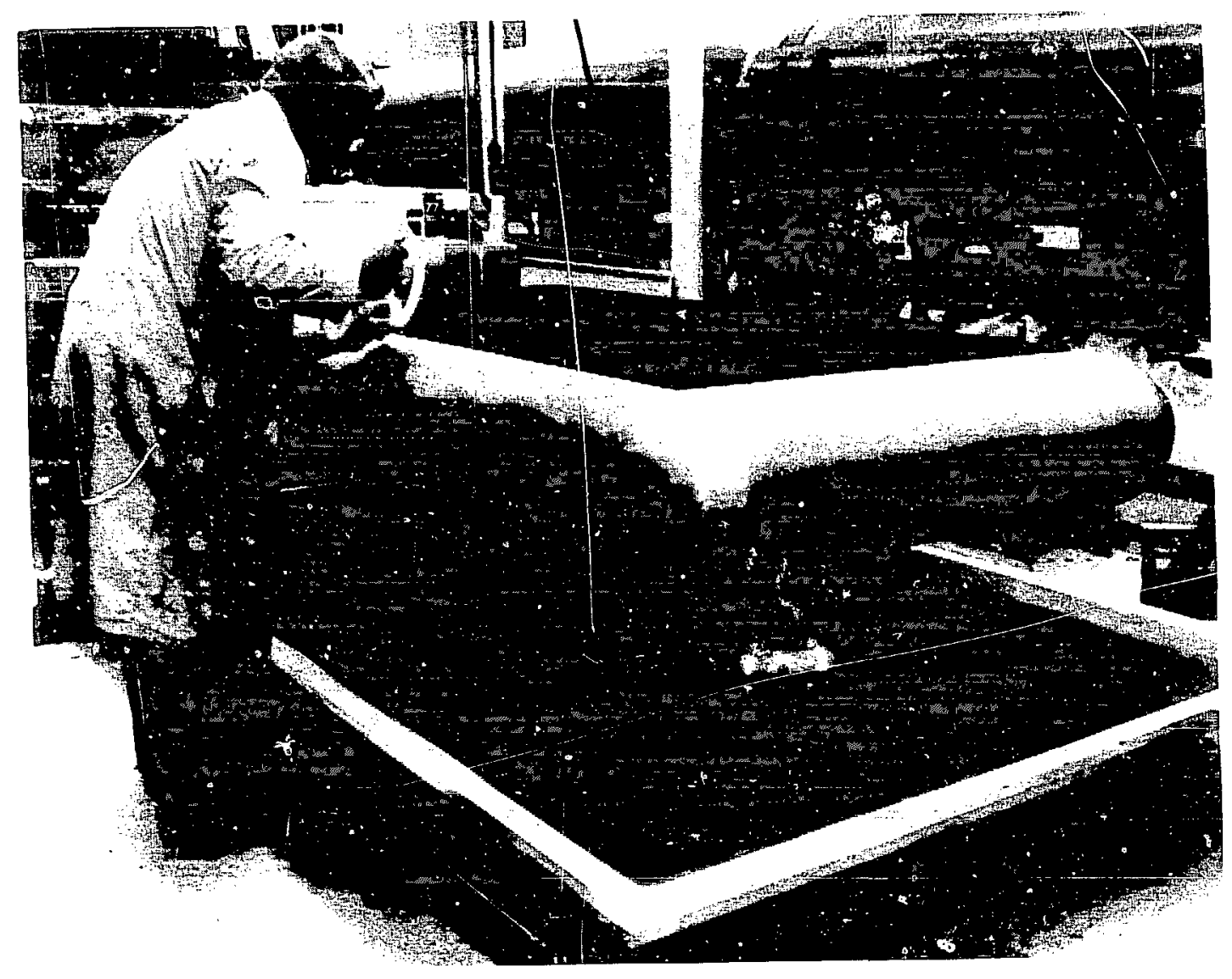

Fig. 7. The KrF laser system's final amplifier, thr LAM, under construction. 


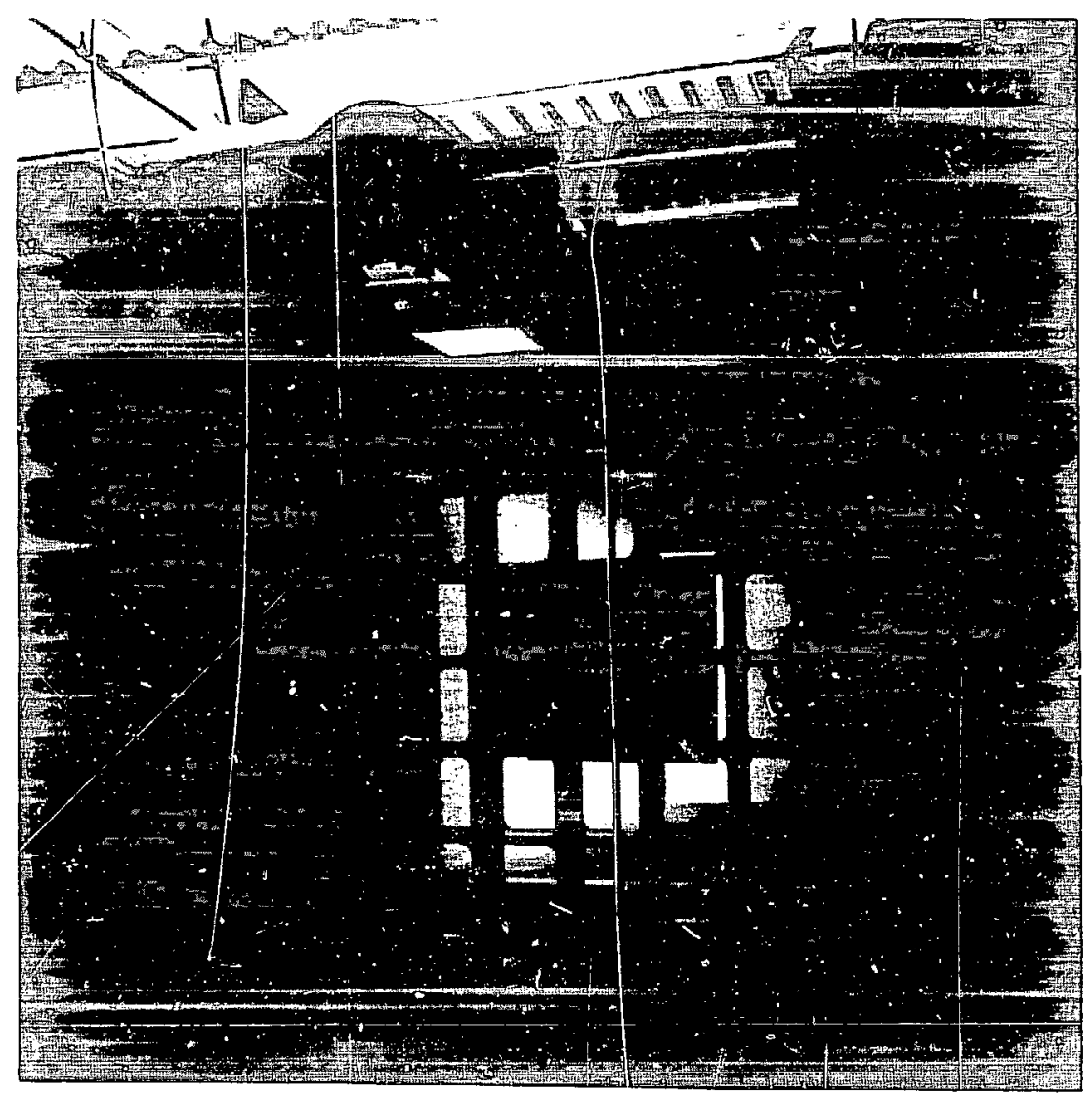

Fig. 8. One of the electron beam cathodes for the LAM with the emitting iviface upright. 


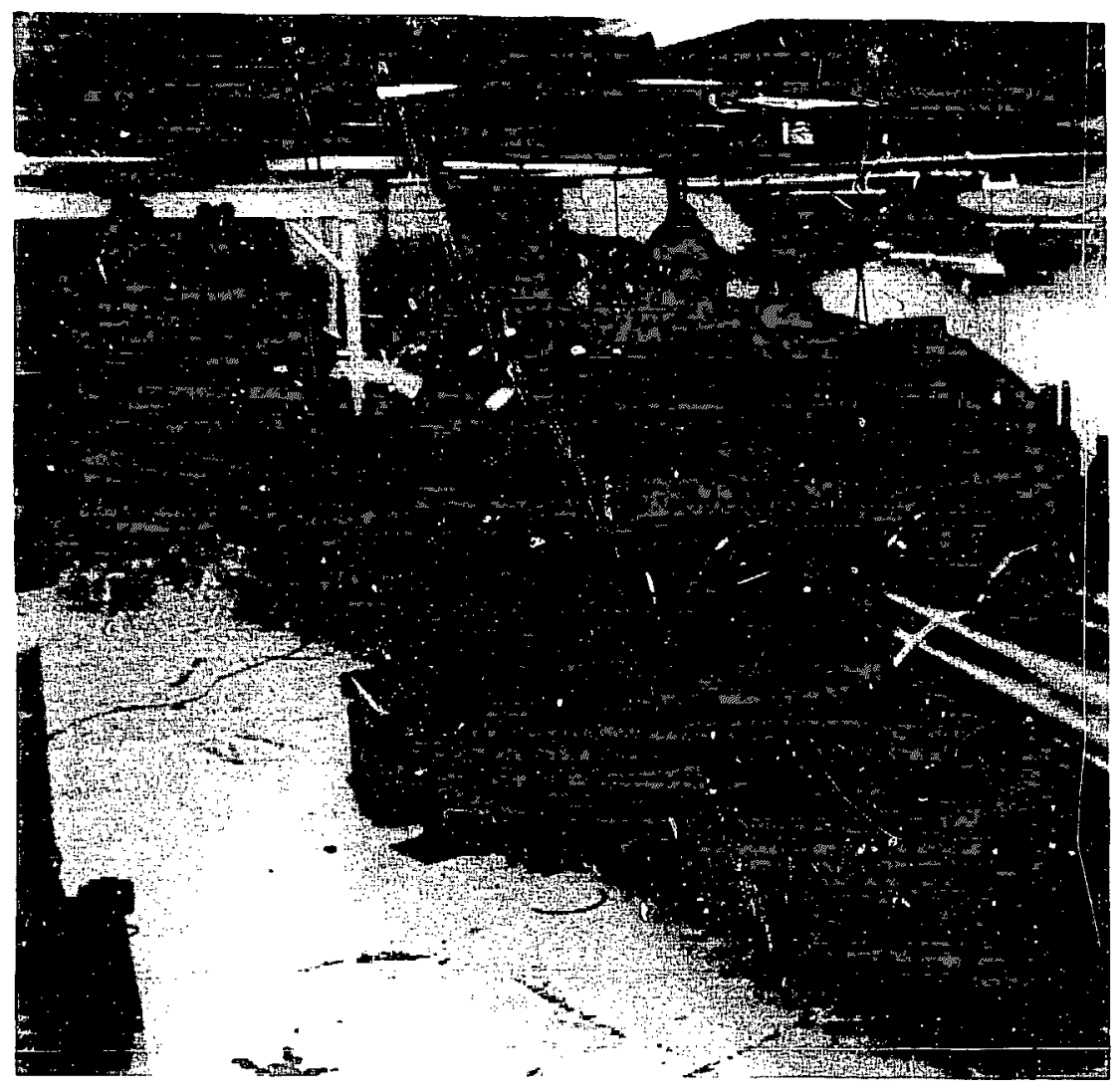

Fig. 9. The LAM being discharged to generate ultraviolet power at a wavelength $\frac{1}{4} \mu \mathrm{m}$. 


\section{REFERENCES}

1. J. E. Velaz:o and D. W. Setzer. "Bound Free Emis sion Spectra of Diatomic Xenon Halides," Journal of Chemical Phl'sics 62, 1990-19991 (1975).
2. J. J. Ewing and C. A. Brau. "Laser Action on the $\Sigma_{i} \rightarrow \Sigma_{i}$ Bands of $\mathrm{KrF}$ and $\mathrm{XeCl} . "$ Applied Phisics Letiers 27, 350-352 (1975). 


\title{
Diagnosis of Laser-Driener Implosions
}

\author{
by Allan Hauer
}

8 ince practical inertial fusion conditiors can occur only in viry highly compressed matter. a thorough understanding of dense imploded plasmas and their production is essential. In this section, a description is given of work that has been done on the diagnosis and modeling of dense plasmas and their creation by laserdriven implosion. Six characteristics of these dense plasmas are of particular interest:

1. Average (electron and ion) density and temperature in the compressed core.

2. Spatial variation of density and temperatures across the core.

3. Temporal variation of core conditions.

4. Velocity history of the implosion. (This parameter is highly coupled to the efficiency with which energy is imparted to the dense compressed plasma.l

5 The temporal history of neutron production and the total yield for DT-filled targets.

6. Integrity (or break up) of the imploding "pusher" shell and its effect on compressed core conditions.

\section{LASER-DRIVEN IMPLOSION EXPERIMENTS AT LOS ALAMOS}

Dense plasma implosion experiments at Los Alamos have been performed with $\mathrm{CO}_{2}$ lasers as the drivers. Most of the diagnostic and modeling techniques that have been developed as part of these experiments are, however, of general applicability to all of ICF irrespective of the driver. Some aspects of this implosion/dense plasma work have actually benefited from the hot-electron ablation of characteristics of $\mathrm{CO}_{2}$ laser drive.

The basic interactions involved in laser-driven implosions are illustrated in Fig. 1. With $\mathrm{CO}_{2}$ lasers, most of the absorbed energy is initially chant aled into a hot electron distribution. The hot electrons are produced near the critical electrun density region for $\mathrm{CO}_{2}\left(n_{\mathrm{e}} \sim 10^{19}\right.$ $\left.\mathrm{cm}^{-3}\right)$, and the ablation occurs in denser reg:ons deeper within the target. The distance between the critical and ablation surfaces is larger for longer laser wavelengths. Scattering of the hot electrons between the critical and ablation surfaces tends to symmetrize and smonth any initial nonuniformities in the laser illumination. The greater distance between the critical to ablation surfaces in $\mathrm{CO}_{2}$ experiments tends to make symmetrization a more prominent effect than in experiments with shorter wavelength lasers.

\section{DIAGNOSTICS FOR DENSE PLASMAS}

Figure 2 illustrates a typical target used in highcompression laser-implosion experiments. Although many other target variations have been tested in the Los A.lamos laser fusion program, this configuration can be used to illustrate many of the relevant aspects of implosion diagnostics.

Within the target is a glass shell filled with a variety of gases that is determined by the experiment of interest. DT is used as the fuel for thermonuclear yield, and spertroscopic diagnosis of plasma temperature and density is accomplished by seeding the DT fuel with a small a mount of higher-Z material. such as necn or argon. Analysis of line emission from the higher: material is one of the most direct diagnostics of com : essed density.

The thick layer of plastic surrounding the glass shell reduces the preheat of the fuel due to suprathermal eiectrons and allows an ablative implosion of the shell. In earlier work of this type ${ }^{1}$ only the thin glass shell was present, and long mean-free-path electrons tended to 


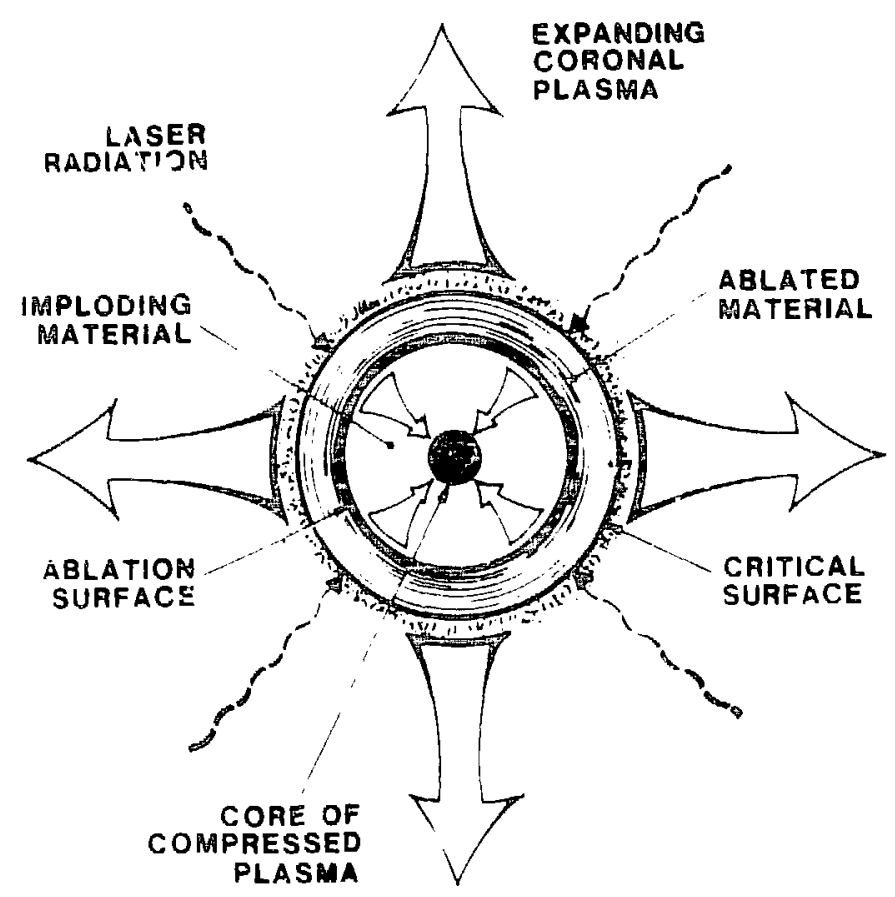

Fig. 1. Basic interactions inwolked in producing laser driter mplosion s.

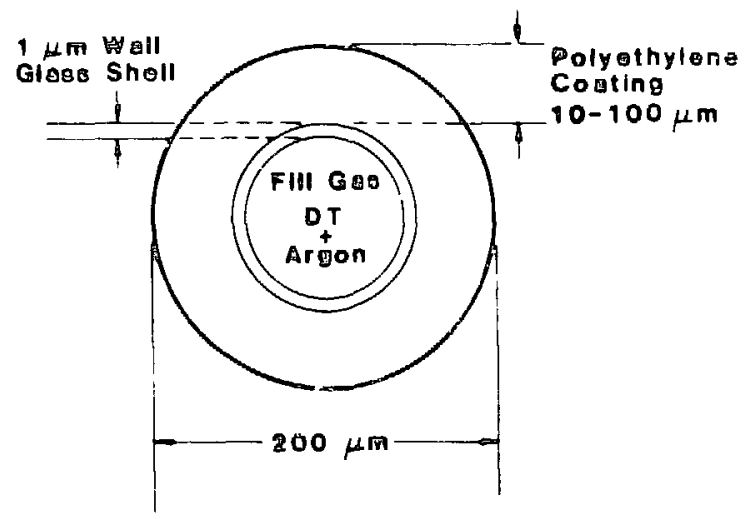

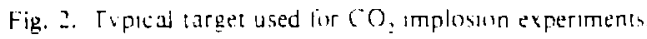

unformly heat the entire shell and cause it to explode. The plastic outer coatung lows the electrons and enables a slower ablative implosion that produces very dense plasmas of a leu $\mathrm{g} \mathrm{cm}^{2}$ density:

In diagnosing much higher density implosions that will be possible in the near future. it might be desirable to construct the gas-containing shell completely out of low. 2 material. It is antsipated that these implosions will reach very high $p R$ 's in the pusher, and the low opactly of plastac shells might still allow for radiation diagnostics of the compression.

The diagnostics of these very dense plasmas fall into live major categories:

1. X ray spectroscopy

2. X-ras imaging

3. Time-resolved $x$ ra! detection

4. Niclear product neasurements

5. Particle (ion) detection

By using information from such a wide variely of measurement techniques. a comprehensive picture of the laser implosion can be formed.

\section{$X \cdot$ Ray Spectroscopy}

Spectroscopic analysis of emission from the higher $Z$ material in the fill gas provides the most comprehensive information on plasma dens. and temperature. The general features of $x$-ray spe " from a laser-imploded plasma when argon was used as the high $Z$ diagnostic trace element are shown in Fig. 3. The three traces represent a progression (top to boltom) to higher densi. ties. Qualitatuely. we see that the lines become hioader 


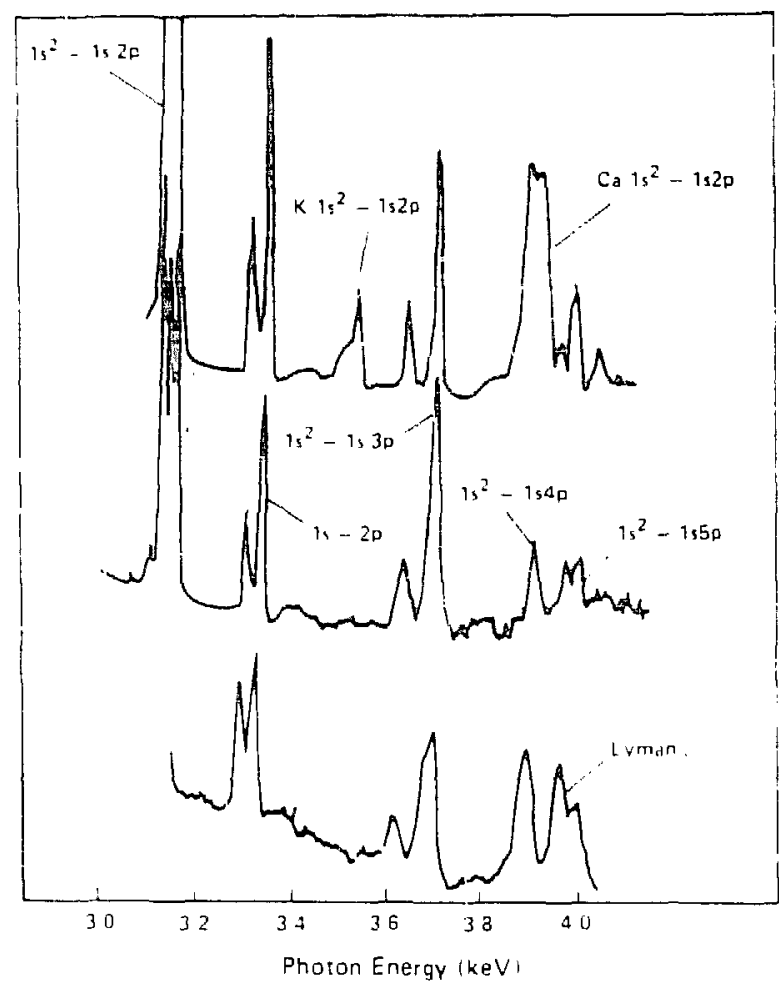

Fig. 3. $X$ iay spectra observed from the implosion of various targets proxducing different tinal densittes.

as the density rises. The top trace was obtained from an exploding pusher target irradiated with a $3.5-\mathrm{kJ}\left(\mathrm{CO}_{2}\right)$ laser energy and represents a compressed electron deilsily of about $9 \times 10^{22} \mathrm{~cm}^{-3}$. The rniddle trace was ubtained from a target with a coating of $25-\mu \mathrm{m}\left(\mathrm{CH}_{2}\right)_{r}$ (and $4.5 \mathrm{~kJ}$ laser energy on target). The compressed electron density here is about $4 \times 10^{23} \mathrm{~cm}^{-3}$. The final trace was produced by a target with $50-\mu \mathrm{m}\left(\mathrm{CH}_{2}\right)_{x}$ coating and about $5.8 \mathrm{~kJ}$ of laser energy. The compressed electron density is about $7 \times 10^{21} \mathrm{~cm}^{-3}$. In the experiment represented by the top trace of the figure. the laser heats the glass shell directly. and lines due to calcium and porissium (which are impurities in the glass) are exciced. When a layer of about $25 \mu \mathrm{m}$ of plastic is added, these Ines are suppressed.

Analysis of the profiles of these lines is one of the primary diagnostics of compressed density. An important part of this analysis is a comparison with theoretical profiles. which are calculated as a function of density for

- See Ref. 3. In addition to the temperature dependence of line profiles. this reference also discusses other aspects of theoretical line profile idculations relevant to plasma density diagnosucs. helium-lile and hydrogen-like emission. Some of the first helium-like profile calculations were performed as part of the Laboratory's implosion diagnostics program. "In. Fig. 4. we show the results of detailed fitting of theoretical profiles to experimental points. Because the $x$-ray line spectra are time integrated. the value of compressed density obtained by this profile fitting represents an average value during the compressed phase.

Electron temperatures are measured by using both "line ratios" and "continuum slopes." The continuum slope method is the most straightforward. although it is someimes complicated by hard $\mathrm{x}$-ray background (due to the fast electrons). When special precautions are taken. temperature measurements of $\pm 50-\mathrm{eV}$ accuracy are possible. Line ratio measurements are complicated by the very dense plasma conditions as the high density causes only a relatively small number of the members of a series to be present while causing the low-quanum-number transitions to be optically thick. This usually makes line ratios between ionization stages of a given species the best choice for determining the electron tempiature. This, of course. introduces a dependence on electron density and the self-consistency between the density and the temperature must be carefu!ly checked.

Time-integrated $x$-ray spectra (in this energy range) are measured using crystal-dispersurg spectrographs. For example. the spectra in Fig. 3 were taken with a PET crystal whose (002) reflection planes have a 2D spacing of $\AA .74 \AA$ A.

Spatial resolution orthogonal to the dispersion (provided by a slit) allows accurate determination of the spatial distribution of emission. Careful characterizations have been made of the instrument profiles and response functions of such measurement systems as well as the effects of the spatial distribution of the radiating sources. Such information is carefully accounted for in comparing the measurements with theoretical modeling.

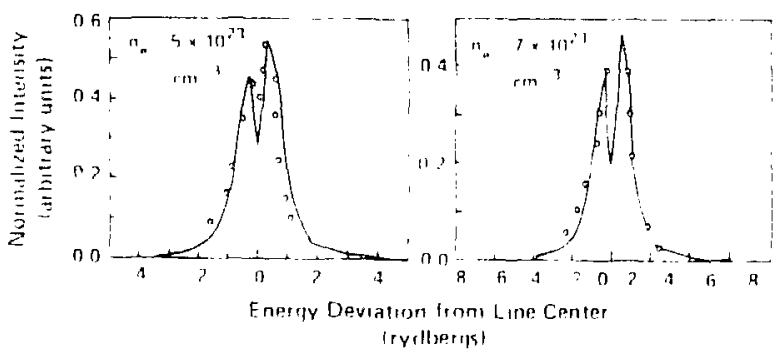

Fig. 4. Plasma density measurements are obtained from the fitung of theoreucal line profiles to experimental points. 
In the period around peak compression, most of the pusher is relatively cold $(\sim 300 \mathrm{eV})$ and derse. Continuum radiation from the core thus passes jut through a relatively long path in material that is considerably colder than the fill gas, which produces absorption features in the spectrum. Analysis of these features can be used to determine the conditions in the pusher and can be important in overall characterization of the implosion. In Fig. 5, we show such an absorption spectrum produced by silicon ions in the glass.

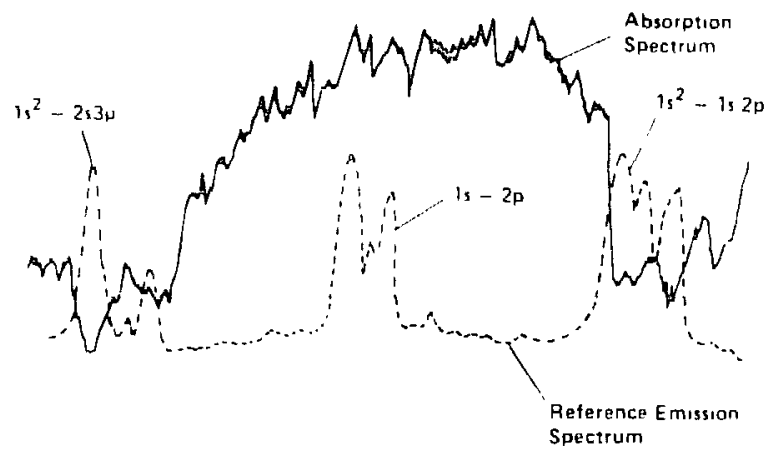

Fig. 5. Silicon absorptoon spectrum.

In the sectiun below, on the stability of laser driven implosions, we show how more detailed information can be obtained from absorption spectra.

\section{$X-R$ ay Imaging}

The primary auxiliary method for the measurement of density is the determination of radiation source sizes by $\mathrm{x}$-ray imaging. As mentioned above, the emission from the compressed core is usually spatially resolved by placing a slit perpendicular to the plane of iricidence on the crystal. This produces one-dimensional spatial resolu tion orthogonal to the dispersion direction. Unfolding techniques ${ }^{5}$ have been developed that allow determina tion of the source distribution. These techniques account for the integration along the line of sight and also take into account the ?nite size of the slit. By spatially resolving several opticaliy thin lines. we can obtain a rough indication of the temperature variation within the compressed core. Knowing the average diameter of the compressed material and the initial fill pressure, we may determine a compressed density.

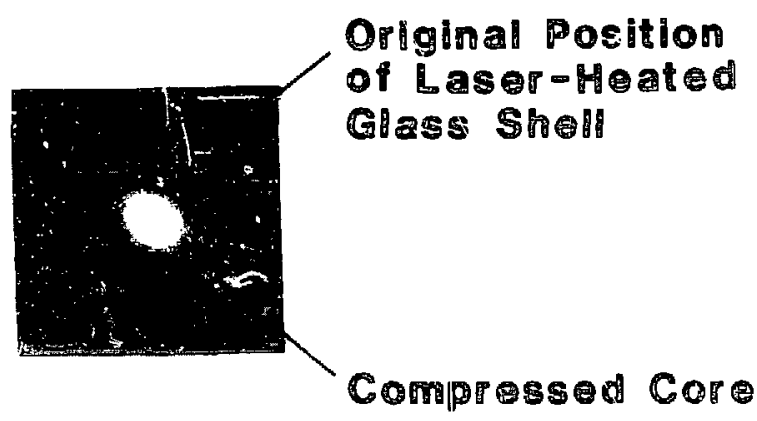

Fig. 6. X-ray image of a laser-driven implosion.

Broadband iwo-dimensional $x$-ray imaging of the compressed core can be performed with pinhole cameras or $\mathrm{x}$ ray microscopes. In Fig. 6, we show an example of such an image. These images are primarily useful in diagnosing the two-dimensional symmetry of the implosion. Implosion symmetry by itself is an important implosion characteristic that will need to be studied in great detail the future.

By selective filtering of the images, gross indications of the temperature and density gradients in the compressed core can be obtained. These images do reveal one interesting characteristic of $\mathrm{CO}_{2}$ laser-driven implosions That is, even with significant non-uniformiiy in the illumination, the hot-electron symmetrization effect tends to produce rather symmetric compressed cores.

\section{Time-Resolved X-Ray Detection}

Time resolution of broadband $\mathbf{x}$-ray emission gives useful information on the velocity characteristics of the implosion. The subnanosecond time resolution required can be obtained with fast $x$-ray diodes or with an $x$-ray streak camera. In Fig. 7, we show an x-ray streak photo of an imploding target. In this particular case, the target was a thin (unfilled) gold shell irradiated with about $5 \mathrm{~kJ}$ of energy from the Helios $\mathrm{CO}_{2}$ laser. The iong pulse of soft radiation comes from the heating and ablation of the shell. The short burst of somewhat harder radiation comes from the imfiusion when the shell collapses to a compressed core.

Information such as this can be useful in characterizing the velocity time history of the implosion. Such information is particularly important as very high compressions are reached and stability questions become especially important. 
X-RAY STREAK PHOTOS

OF LASER IMPLOSION

OF GOLD SHELL

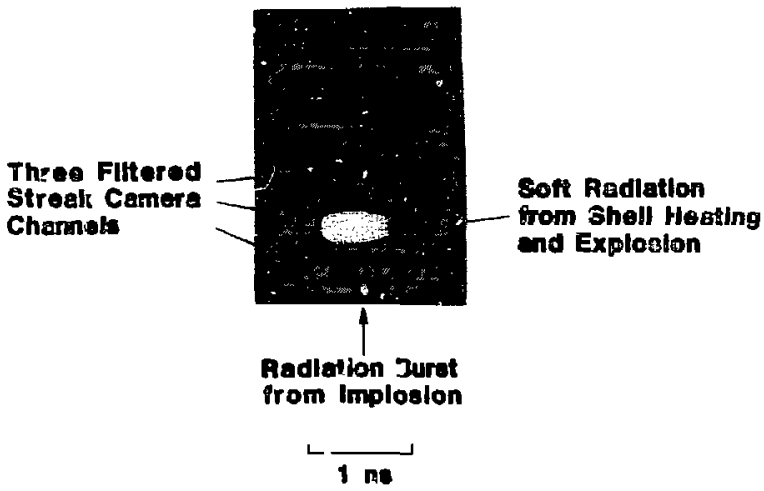

Fig. 7. Sucak measurement of the temporal profile of $x$-rays from a laser-driven implosion.

\section{Nuclear Product Measurements}

Yield from thermonuclear reactions is, of course, the end-product goal of ICF. When targets, such as that shown in Fig. 2, are filled with DT, significant thermonuclear neutron yield can be achieved. For example, on the Helios laser system with about $5 \mathrm{~kJ}$ on target, yields of near $10^{9}$ neutrons per shot were achieved.

Neutron yield measurements can be used to provide an estimate of compressed density. The yield can be written

$Y=n_{\text {ion }}^{2}\langle\sigma v\rangle V \Delta t$

where

$\mathrm{n}_{\mathrm{ion}}=$ compressed ion density,

$\langle\sigma v\rangle=$ velocity-averaged cross

section for DT reactions,

$\Delta t=$ reaction or confinement time, and

$\mathrm{V}=$ reaction volume.

Unfortunately, in the temperature range in which we are presently operating, $\langle\sigma \mathrm{v}\rangle$ varies roughly as $\mathrm{T}_{\text {lon }}^{6}$. Inaccuracy in the measurement of $T_{\text {ion }}$ thus (at present) makes the density determination only an order-of-magnitude estimation. It is, however, a very useful cross check to compare with more accurate density estimates from spectroscopy.
Time-resolved measurements of neutron production can provide inportant information on the characteristics of the implosion. For example, the timing of the peak of neutron production with respect to the rise of the laser can give an estimate of the implosion velocity. In Table I, we shlow measured laser rise-neutron delays as a function of plastic thickness for targets like that illustrated in Fig. 2. Total energy on target was about $5.5 \mathrm{~kJ}$. These correspond to implosion velocities between $8 \times 10^{6}$ and $3 \times 10^{7} \mathrm{~cm} / \mathrm{s}$. The width of the time-resolved neutron signal can also be used to estimate the ion temperature of the compressed core.

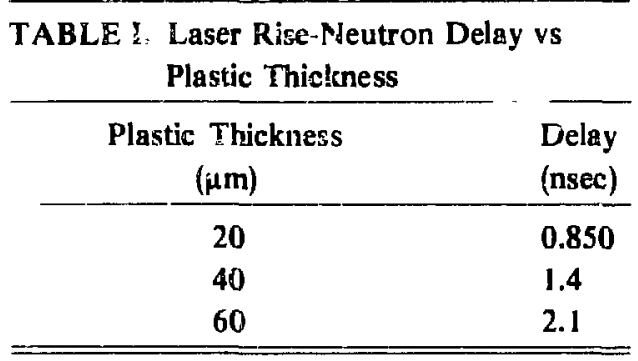

\section{MODELING OF LASER-DRIVEN IMPLOSIONS}

Numerical hydrody ramic codes are the primary methods used in analyzing these implosions. It is important to use these codes to simulate experimental diagnostic output. A strong interaction between theory and experiment can thus be established, which is of benefit to both.

Most implosion simulation work has been done with the Lagrangian hydrodynamic code LASNEX. ${ }^{6}$ NonLTE (local-thermodynamic-equilibrium) ionization dunamics and radiation transport are handled in real time (that is, in line with hydrodynamic variations) with an average ion model. Detailed radiation behavior, such as transport of an individual line profile, is handled in a postprocessing mode. In other words, we assurne that the detailed radiation behavior does not affect the overall dynamics of the problem. The output of temperature and density data is then used to calculate particular radiation information.

When we attempt very detailed comparisons between theory and experimer,t for laser plasmas, the question of non-LTE behavior arises. Because $\mathrm{x}$-ray diagnostics are a 
primary source of information on implosion dynamics, it: is important to carefully model these radiation signatures.

The following simple considerations indicate the importance of non-LTE analysis in predicting radiation signatures. For a level to be in collision-dominated (CD) equilibrium with higher levels, we ignore pressure ionization effects and use the following criterion taken from Griem :'

$N_{e}>7 \times 10^{18} \frac{Z^{6}}{(n)^{22}}\left(\frac{k T_{e}}{Z^{2} E_{H}}\right)^{1}{ }^{2} \mathrm{~cm}^{-3}$.

where $N_{e}$ is the electron density $\left(\mathrm{cm}^{-3}, \mathrm{n}\right.$ is the principal quantum number. $E_{H}$ is the ionization energy of hydrogen. and $T_{e}$ is the eiectron remperature. For example, for $\mathrm{n}=2$, and $\mathrm{Z}=18 . \mathrm{N}_{\mathrm{e}}>4 \times 10^{24} \mathrm{~cm}^{-3}$, while for $\mathrm{n}=3$. $N_{e}>1.1 \times 10^{23} \mathrm{~cm}^{-3}$. The state $\mathrm{n}=2$ is not in local thermodynamis equilibrium (LTE) with higher levels in past $\mathrm{CO}_{2}$ laser imr!osion experiments (such as those utilizing targets like that shown in Fig. 1). while $n=3$ is usually close. The helium- and hydrogen-like resonance lines often have a large oftical depth. which brings $n=2$ much closer to LTE than it would otherwise be (through absorption fi:on the ground state). Clearly, to deal with questions such as the detailed transport of optically thick lines (for exampie, Lyman $\alpha$ and $1 s^{2}-1 s 2 p$ in Fig. 3), a detailed non-LTE radıation model must be utilized.

In order to calculate the radiation from these laserdriven implosions, an extensive collisional-radiative model has been constructed." The time- and space varying nature of the emitting region is included. although the final comparison is with a time-integrated spectrum. By calculating a large number of spectral features (line intensities, profiles, etc.), we can obtain a substantial confirmation of plasma conditions. The collisional-radiative model incorporates overall plasma conditions that are predicted by a one-dimensional version of LASNEX. Experimental measurements provide a substantial number of constraints on the choice of input concitions to these calculations. These measurements included ( $a$ ) therm nnuclear yield measurements. (b) ion temperature obtáı ıed from neutron temiporal broadening. (c) implosion times from time-resolved neutron signals.

- In collaboration with the Plasma Radiation Group at the Vaval Research Laboratory. (d) hot-electron temperature measurements, and (e) absorbed energy measurements. The calculational procedure is thus composed of two steps. LASNEX calculation of the overall plasma conditions (where experimental measurements affect the choice of LASNEX iritial conditions) constitutes the first step. In the scoind stêp, detailed radiation characteristics are calsulated with the coilisional-radiative (CR) model (using LASNEX-calculated input conditions). A flow diagram illustrating the calculational procedure is given in Fig. 8 .

An example of the results of such modeling is given in Fig. 9 for a target quite sirnilar to that shown in Fig. 2 that was irradiated with about $4 \mathrm{~kJ}$ on the Helios laser system. The final compressed electron density was about $3.5 \times 10^{23} \mathrm{~cm}^{-3}$. The neutron yield was about $2 \times 10^{8}$.

Detailed comparisons between theorelical modeling and experimental measurements for a variety of diagnostics such as imaging, spectroscopy. and nuclear measurements can provide a comprehensive picture of laser-driven implosions. ${ }^{8}$ In future experiments, it will be vital to continue this pattern of detailed comparison and feedback between theory and experiment.

\section{STABILITY OF LASER-DRIVEN IMPLOSIONS}

One of the most crucial issues for the ulrimate success of ICF is the stability and uniformity of the imploding pusher shell. The uniformity could be disrupted by classical hydrodynamic instabilities (such as RayleighTaylor) that occur at the interface between different density materials. Disruption of the symmetry could also be caused by other effects such as nonuniformities in the ablation drive.

Several preliminary experirnents have been performed to deyelop diagnostics of shell stability. The experiments can be divided into two general categories: acceleration and deceleration phase experiments that study break up and instability early in the implosion and near stagnation. respectively.

\section{Acceleration Phese Stability}

The acceleration phase work lias been done with cylindrical-shell targets that have been imploded with relatively uniform eight beam $\mathrm{CO}_{2}$ laser illumination. Typically, about $5 \mathrm{~kJ}$ was incident on taiget. Cylindricai 


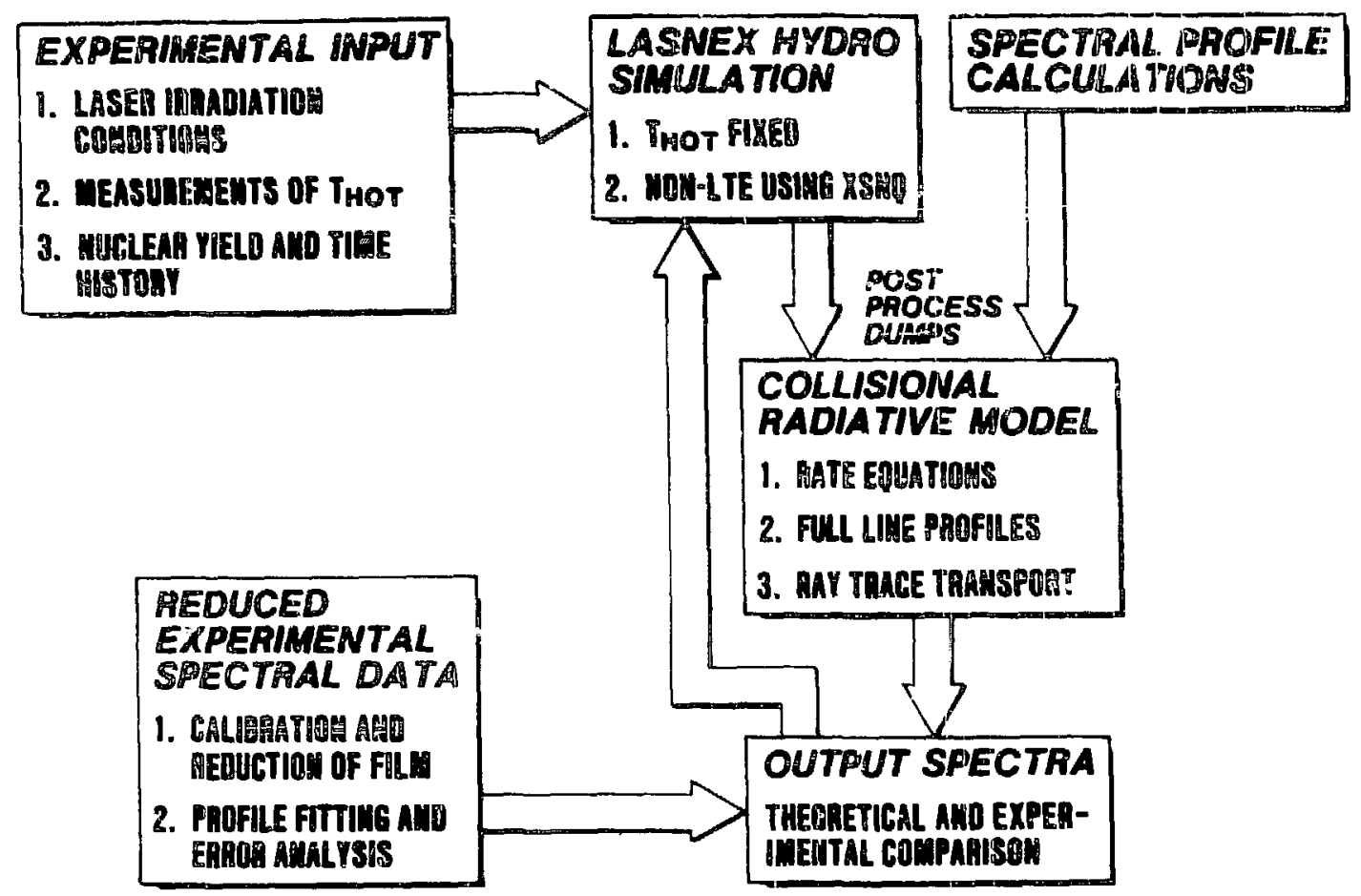

Fig 8. Flow diagram of the calculational procedure used in simulating $x$-ray signatures of laser imflosions.

configurations offer many advantages in stability experiments. The open geometry facilitates diagnosis while testing the: eftects of implosion convergence. The cylindrical geometry is also convenient for comparison with some types of theoretical modeling.

In the present work, the targets were cylindrical shells of copper and aluminum with and without plastic coating. The basic carget configuration is illustrated in Fig. 10 . The targets were about $200 \mu \mathrm{m}$ in diameter with walls about $2 \mu \mathrm{m}$ thick. The uncoated shells behaved much like explosive pushers, whereas the plastic-coated targets behaved similarly to the quasi ablative targets used in the implosion experiments described earlier. ${ }^{2}$ The eight beams were independently targeted (and defocused) around the cylindrical surface of the targets.

In addition to the simple shills, some of the targets had initially imposed perturbations, as shown in Fig. 11 , which were arranged to maintain a constant wall thickness. The period of the perturbations was about $25 \mu \mathrm{m}$ with an amplitude of $0.5 \mu \mathrm{m}$. The axial perturbations (Fig. 11a) are compatible with the cylindrical symmetry in LASNEX, which permitted the results from LASNIEX calculations to be used for some preliminary scoping of the types of grow'th expected.
The implosions were diagnosed primarily with $x$ imaging of self-emission. Several pinhole cameras . arranged to provide imaging along, ind perpendicula the cylindrical axis. In Fig. 12, we show a compar between the implosions of coated and uncoated $c$ : drical shells (viewing along the cylindrical axis). $m$ from images that were filtered for ibout $1.5-\mathrm{keV}$ ra tion. The comparison here is very similar to the explc versus semiablative studies done in the high-density sc of spherical implosion experiments. Also shown in 12 is an image taken with a view perpendicular to axis, which confirms that it is possible to obtai relatively uniform implosion all alon; the cylindrical (that is, the views in Fig. 12a and 12b are not end effe

We now turn to the targets that contained prearrar perturbations. Targets that contained perturbations : always cnated with plastic so that perturbed ve: unperturbed comparisons are only nade for the st ablative case. In Fig. 13, we show a cornparison of 6 on pinhole camera views for unperturbed and pertur cases. In all cases where perturbations were present. compressed core is significantly larger (weaker $c_{1}$ pression) and is surrounded by a halo of radia material that, in many cases, contains a periodicity. 


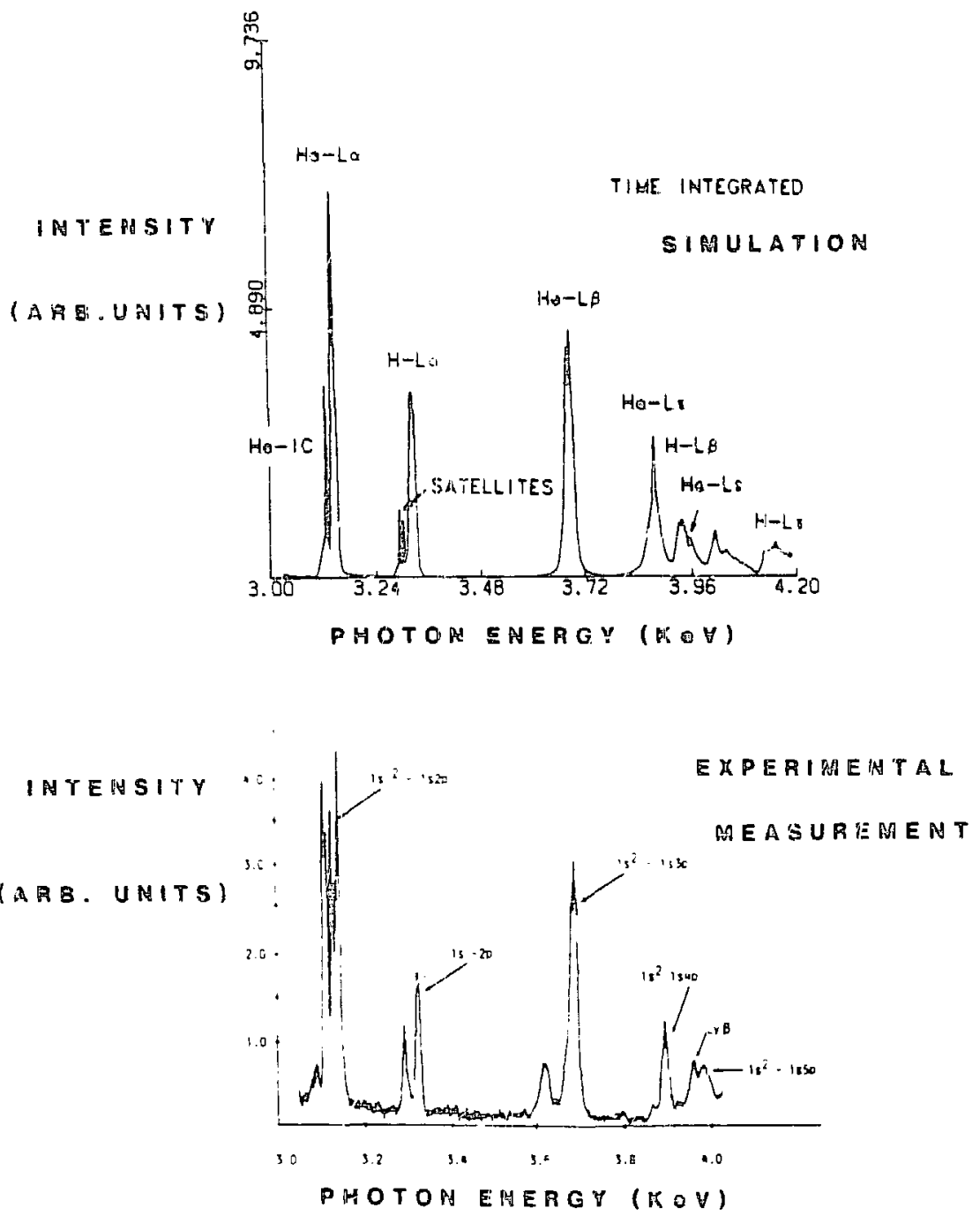

Fig. 9. Comparison tetween theory and expermental of time-integrated ray specium. 


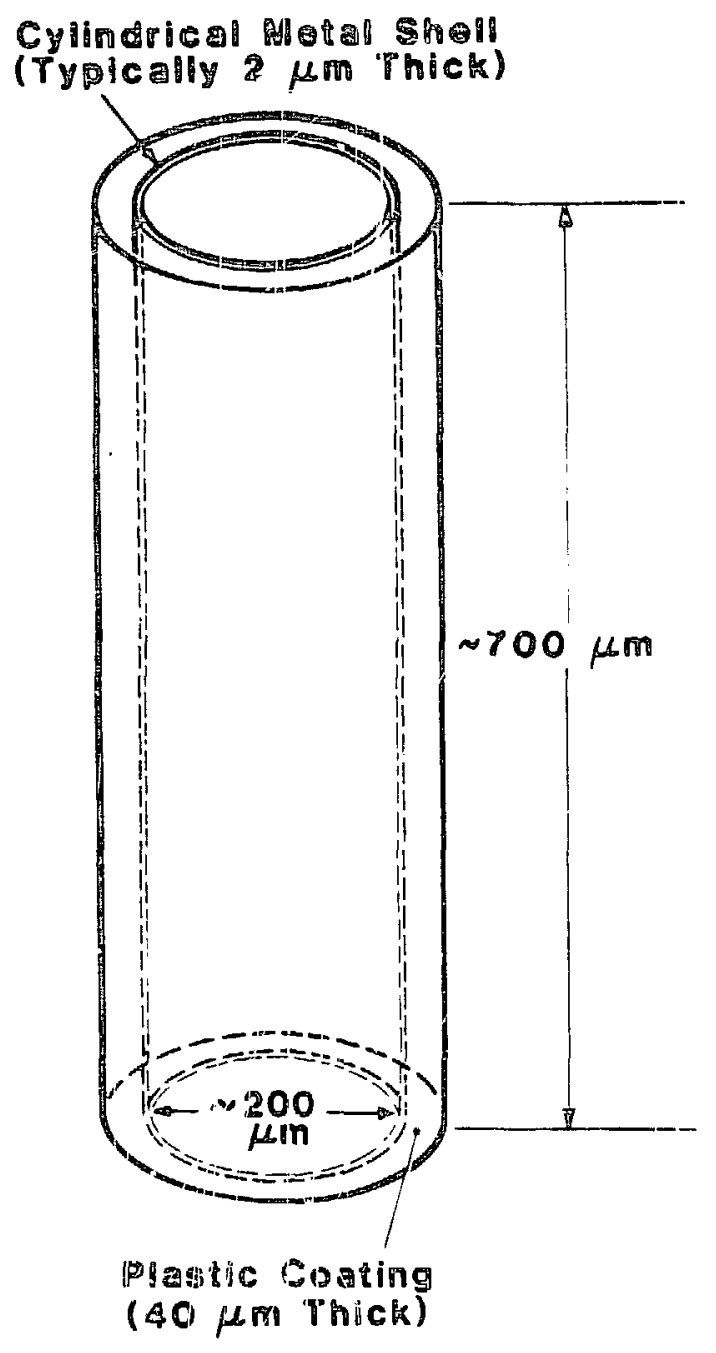

Fig. 10. Basic arget configuration used in cyhndricol stability experiments.

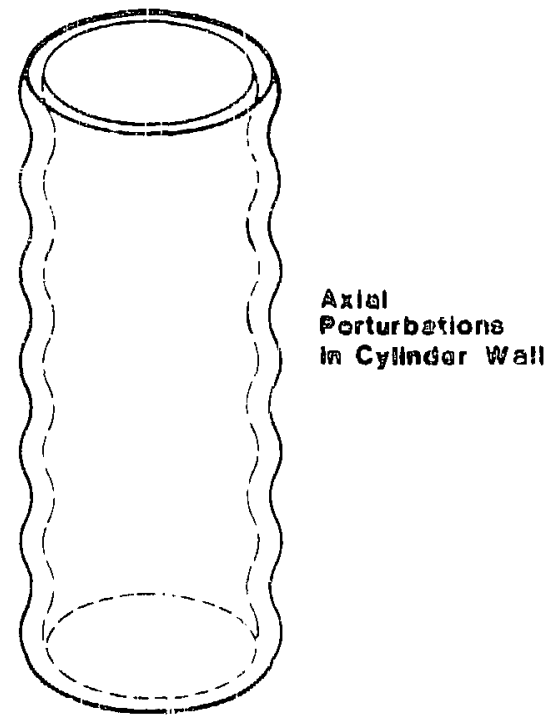

Fig. il. Prturbauons imposed on the cylindrical ta:izets.

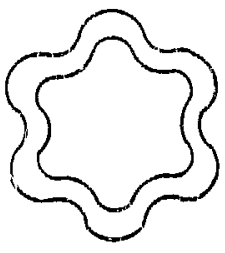

As iwathal Porturbotions In Cyltnder Wall

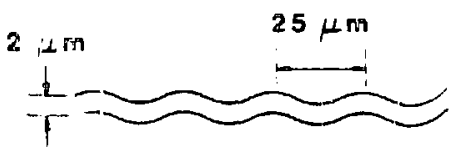

Structure of Perturbetione (Af aintains Conetant

Thickness) 


\section{X-RAY MAGE OF ABLATIVE YS EXPLOSUVE}

IMPLOSIOA OF CYLIRDRACAL SHELLS

Thin Shell

(Explosive)

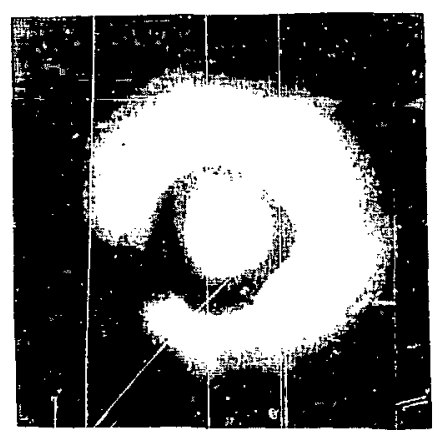

View Along Axis

(a)

\section{Plastic Coated Shell \\ (Ablatly}

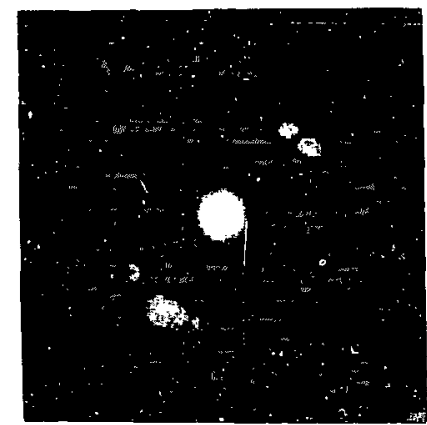

View Along Axis

(b)

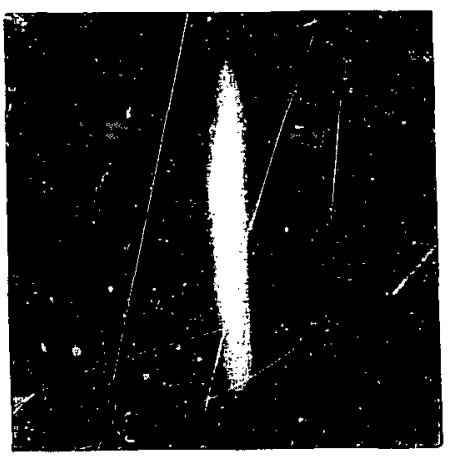

View Perpendicular to Axis

(c)

Fig. 12. X ray imaes of the implosion of coated and uncoated cylindrical shells. 


\section{X-RAY IHAGES OF ABLATIVE CYLINDRICAL 1 A月PLOSIONS MITH AND YYTHOUT BMPOSED PERTUIRRATIONS}

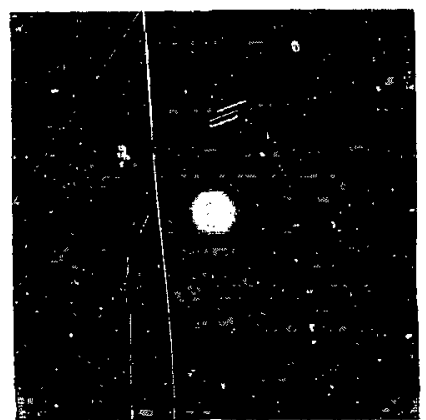

Without Perturbations

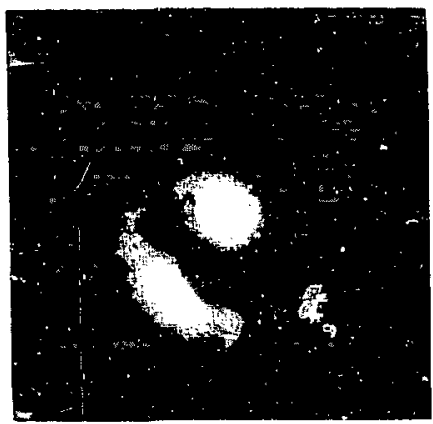

With Perturbations

Fig. 13. X-ray images of the implosion of coated and uncoated cylindrical shells.

print in Fig. 13 does not adequately show this periodicity. The nature of this structure is being analyzed further.

The basic conclusions from these preliminary experiments are as follows:

- Strong, uniform cylindrical implosions can be produced. The $\mathrm{CO}_{2}$ laser is actually an advantage here, giving added symmetrization.

- Evidence of shell breakup was observed in the case of initially perturbed cylindrical targets.

- Cylindrical configuration experiments offer a number of advantages in stability/shell breakup experiments.

\section{Comprassion Phase Stability}

The compression phase stability studies were performed on targets that are quite similar to that shown in Fig. 2.

This series of experiments was designed to be a first test of diagnostic techniques appropriate for studying the breakup of imploding shells. Figure 14 shows the basic target configuration used in these tests. Glass shells (atout $350 \mu \mathrm{m}$ in diameter) were filled with about $12 \mathrm{~atm}$ of DT. In some of the targets, the DT was seeded with about 0.1-0.2 atm of argon. Some of the shells were then

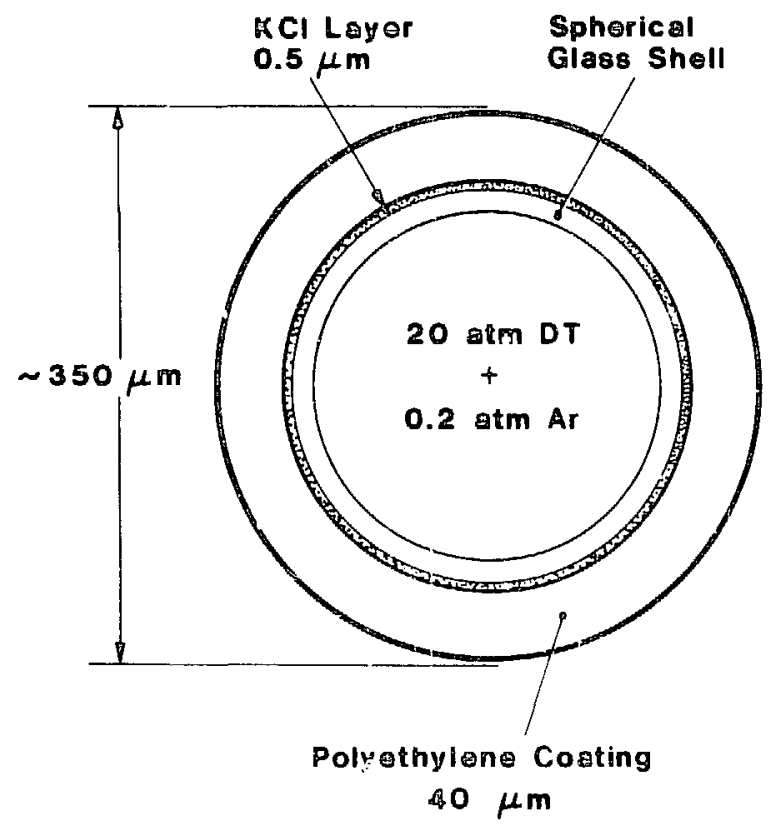

Fig. 14. Target configuration used in spherical shell break up experiments. 
coated with about $05 \mu \mathrm{m}$ of potassium chloride. Other targets contained no potassium chloride coating but were fabricated from glass shells with a high content of calcium and potassium. Finally, the targets were coated with about $40 \mu \mathrm{m}$ of plastic, which made them similar to the targets used in the high-density series described earlier.

When suck targets are laser imploded. there are typically large temperature gradients in the compressed cores. These gradients can be utilized in the diagnosis of shell stability.

Spectroscopic observation of potassium and chlorine radiation served as the signature of shell breakup. These species could only radiate if they penetrated into the central high-temperature regions of the compressed core. If the potassium chloride remains in the outer portions of the shell. previous experimental measurements and theoretical calculations show that it would be at a temperature of about $250 \mathrm{eV}$. This is too low a temperature for efficient excitation of hydrogen-or helium-like potassium and chlorine lines. The argon lines are used to accurately characterize the compressed core conditions. With careful spectral analysis. this information will allow a determination of the amount of shell material mixed into the core.

The targets were chosen to be slightly larger than those in the high-density series. and this produced a weaker compression. Because in this series we were primarily interested in development of the measurement technique, we wanted a larger compressed core that could be more easily diagnosed (for example, easier x-ray imaging).

The eight beams of the laser were focused $300 \mu \mathrm{m}$ hevond center. giving a $T_{\text {hor }}$ (determined from $x$ rays) of ahout $100 \mathrm{keV}$. In Fig. 15. we show a typical spectrum obtained in these experiments. The argon lines show an implusion of modest density $\left(\mathrm{n}_{\mathrm{e}} \sim 2-3 \times 10^{23} \mathrm{~cm}^{-3}\right)$ witt a temperature of about $600 \mathrm{eV}$. This temperature would be more than adequate to strongly excite chlorine lines. The most striking feature of the spectrum in Fig. 15 is. however, the strong chlorine and potassium absorption features. These features indicate that most of the poiassium chloride has remained in the cold outer regions of the sheil: in other words, there is little evidence of shell breakup. The spectra remained very similar to that in Fig. 15 throughout this experiment. In Table II. we sum marize the parameter variation present in this series of experiments. Over the range of parameters in Table II. no evidence of breakup was observed.

Some evidence of breakup was observed in the follow. ing case:

- The target had no potassium chloride coating but had a high concattration of potassium in the glass shell itself.

- There was significant laser beam imbalance.

In Fig. 16 we show an example of this case. and a definite potassium emission feature is noted. There is also some evidence of weak absorption features to the left of the potassium line. Potassium would thus have been seen in both absorption and emission, which indicates partial shell breakup. This is the type of information that we hope to quantify in future experiments.

The initial aspect ratso (pusher thickness/diameter) in these experiments was relatively low $(\sim 4)$. In future experiments we should strive to increase this. This may require replacing the plastic layer with a thin metal laver (to increase the aspect ratio). This, in turn, may favor the use of backlighting to diagnose the compressed core.

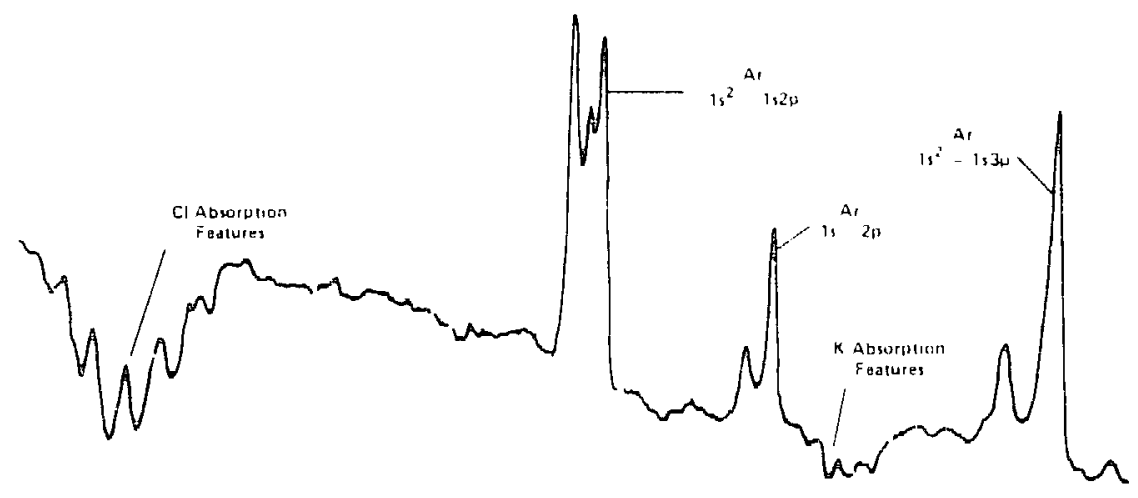

Fig. 15. Typical x ray spectrum ohtaned from the implosion of a target similar to that in Fig. it. 


\section{TABLE II}

\section{EXPERIMENTAL PARAMETERS}

\author{
Inner (glass) shell $0.8 \mu \mathrm{m}<\mathrm{t}<1.8 \mu \mathrm{m}$ \\ wall (t) \\ No. laser beams (b) $6<b<8$ \\ DT gas fill pressure $8<P<15$ \\ $P(a t m)$
}

The Use of Backlighting and Absorption Spectroscopy

In the diagnosis of very dense implosions, the temperatures may sometimes be inadequate for self. emission to be a useful diagnostic. In such cases it may be possible to use an external source of radiation (such as another laser-irradiated target) to backlight the imploding target). In a more sophisticated sense. it may also be possible to use an external source of radiation to perform absorption spectroscopy and obtain more detailed information than could be obtained with a simple shadowgraph. This possibility is in fact already demonstrated by the spectrum shown in Fig. 15.

The absorption spectra represent "self-backlighting," with radiation from the core passing out through the cold absorbing layers. The information is, however, the same as would be obtained if the probing radiation were from a completely external source. As an example of the type of information that can be obtained from spectra such as that in Fig. 15. we consider a simple analysis to obtain the average $\mathrm{pR}$ of the potassium chloride layer.

For one of the absorption feat ses (which corresponds to a particular ionization state) we can write:

$i \mathrm{~K}_{r} \mathrm{~d} v=\frac{\pi \mathrm{e}^{2}}{\mathrm{mc}} \mathrm{Nf}$.

$$
\begin{aligned}
& \text { where } \\
& \begin{aligned}
K & =\text { absorption coefficient } \\
V & =\text { number of ions in the gruund state, and } \\
\mathrm{f} & =\text { average oscillator strength for the transitions in- } \\
& \text { volved. }
\end{aligned}
\end{aligned}
$$

We note that

$I=I_{0} e^{-K R}$.

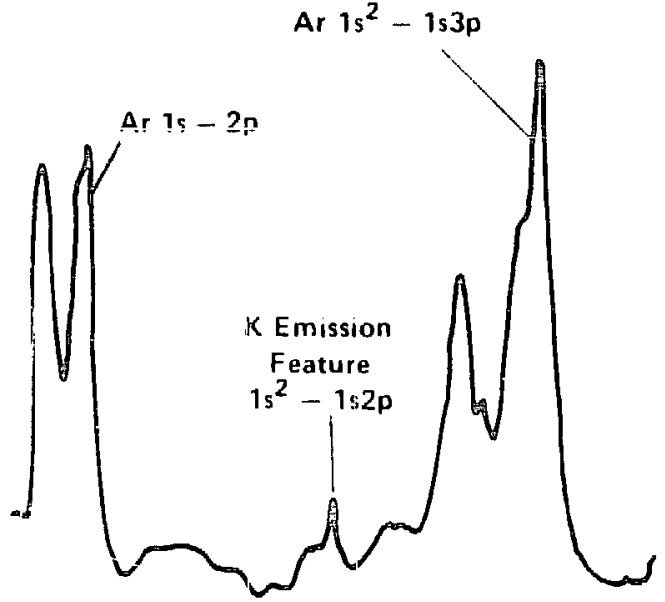

Fig. 16. $\mathrm{X}$-ray spectrum showing, potassium emission feature.

where $\mathbf{R}$ is the path length through the absorbing medium.

Combining Eqs. (3) and (4), we can write approximately

$\left[\ln \left[\frac{I_{0}(v)}{I(v)}\right] d \nu \cong \frac{\pi e^{2}}{m c} f[\rho R]\right.$.

Using Eq. (5) for the spectrum in Fig. 15, we obtain an average value of $\rho R$ of about $10^{-4} \mathrm{~g} / \mathrm{cm}^{2}$. This is consistent with values predicted by hydro simulation.

In the near future, absorption and backlighting techniques should provide a wealth of implosion diagnostic information.

\section{SUMMARY}

Techniques have been developed for measuring many characteristics of the very dense plasmas produced in laser-driven implosions. Measurements have been $\mathrm{com}$. pared with theoretical modeling to provide an accurate picture of these implosions.

Methods are currently under development for addressing the crucial question of implosion stability. one issue that is central to all of inertial confinement fusion. Freliminary tests of some aspects of these ideas have been successful. 
In the future the density of these implosions will increase and aiterations and extensions of the diagnostic methods discussed here will be required. One of these extensions will involve the use of an auxiliary laser plasma x-ray souree to backlight and probe the compressed plasma.

\section{REFERENCES}

1. B. Yaakobi. [1. Steel. E. Thorsos, A. Hauer. and B. Perry, Phis. Rev. Lett. 39. 1526 (1978).

2. A. Hauer, K. Mitchell. D. Van Hulsteyn. T. Tan, F. Linnebur. M. Mueller. P. Kepple. and H. Griem, Fhy's. Rev. Lett. 45. $1495(1980)$.

3. A. Hauer, in Spectral Line Profiles (de Gruyter. Berlin. 1481).

4. P. C. Kepple and K. G. Whitney. U. S. Naval Research Laboratory Report No. 4565 (unpublished) performed under contract to Los A.lamos National Laboratory.
5. M. M. Mueller, Bull. Am. Phys. Soc. 24, 1053 (1979). and Los Alamos Scientific Laboratory document LAUR-78-2698 (1978).

6. G. Zimmerman and W. Kruer. Comments Plasma Phy's. Controlled Fusion 2, 85 (1975).

W. A. Lokke and W. H. Grasberger, Lawrence Livermore National Laboratory report UCRL 52276 (unpublished).

7. H. R. Griem. Plasma Spectroscopy (MeGraw-Hill. New York. 1964) (available from University Microfilms!

8. A. Hauer. K. Mitchell. D. Van Hulsteyn, and E. S. Linnebur, "Comprehensive Analysis of High Density $\mathrm{CO}_{2}$ Laser Driven Implosions Experiments." Los Alamos National Laboratory memorandum P-4-4/29/80/-189. 1980: A. Hauer. K. Whitney. P. Kepple. and J. Davis. Phis. Rer. A 28, 963 (1983). 


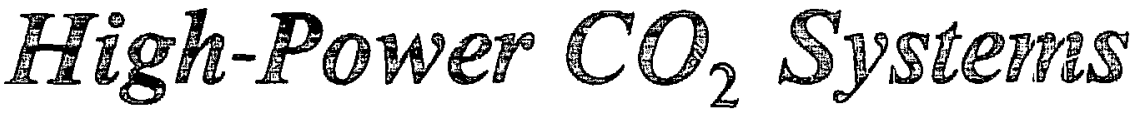

\author{
by C. R. Mansfield and W. H. Reichelt
}

he concept of using short-pulse, high-energy lasers to drive fusion reactions dates back to the early 1960's, but fusion experiments became possible only after the development of large, powerful glass and gas laser systems proved feasible in the late 1960's and early 1970's. A generic laser system (Fig. 1) for fusion experiments consists of five basic blocks: (1) a shortpulse generator with amplifiers in which the short pulse of $0.5-10 \mathrm{~ns}$ and appropriate spectral characteristics is created: (2) the main power amplifier, which amplifies the pulse energy to design points during its transit; (3) the target chamber, which provides the necessary experimental vacuum environment where the optical energy is brought to focus on the target: (4) the optical train: and (5) the control systems that connect the short-pulse generator, power amplifier, pulsed power, target systems, and the optical train into an integrated unit.
The main thrust of fusion laser development at Los Alamos National Laboratory has, until recently, been directed toward $\mathrm{CO}_{2}$ laser systerns. Carbon dioxide lasers are usually operated at a wavelength of about $10.6 \mu \mathrm{m}$, although many other lasing wavelengths are possible. The earliest work at Los Alamos concentrated on the production of $1-n s$ pulses and the amplification of those pulses. The largest uncertainties in the early program centered around the design of large, energetic amplifiers. Two general types of amplifiers arose out of the early work. The difference in the type depends on the means of development of a stable electrical discharge. The first type of amplifier operates with a self-sustained electric discharge. The second type is one in which the electrical discharge is maintained by means of an external source of electrons (electron gun). In the second type, the highenergy electrons injected into the gas produce a stable

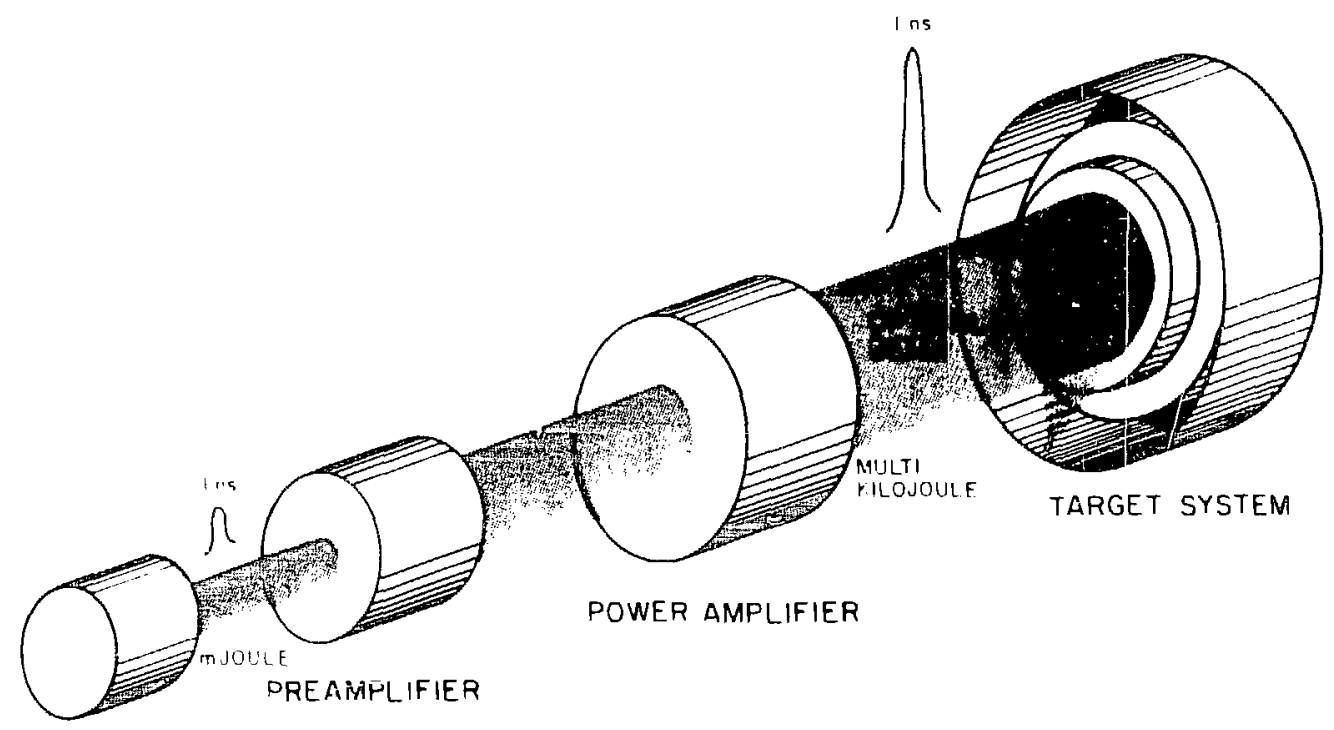

PULSE GENERATOR

Fig. I. The major components of a high-energy laser system. The fifth major component the control system, is not shown. 
low-impedance region of ionization into which energy can be deposited from a second electrical energy source.

The electron-beam (e-beam)-controlled discharge iaser, a technological breakthrough, was invented independently at Los Alamos and at the AVCO Everett Research Laboratory. It was scalable to large apertures and was the most efficient system known. We recognized from experiments and analyses that to scale to large amplifiers, the e-beam-controlled devices were required: hence, our development effort was directed primarily toward electron-beam-sustained devices. Since the selfsustained discharge lasers were efficient and relatively adsy to build and maintain. commercial applications soon became apparent and such lasers quickly became comnercially available. The need for amplifiers in the Anares class of energy outfut $(10-20 \mathrm{~kJ})$ has not found -ummercial application. so this type of laser continues to te specially built for a particular use.

The hardware development effort was augmented by in intensive analytical effort that led to a general under itanding of the physical processes occurring in $\mathrm{CO}_{2}$ laser lischarges. This analytical effort has carried on through he years and has provided the foundation for each iuccessive laser systen designed and built at Los Alanos. The 15-: Los Alamos effort has recently cul ninated in the completion of the Antares laser system. urrently the world's largest operational laser system.
The elements of the electron-beam-sustained amplifier are shown in Fig. 2. Electrons are generated in the gun section either by thermionic emission from hot filaments or by field emission from refractory metal blades. Electrons are accelerated by high electric potentials (200-500 $\mathrm{kV}$ ) and pass through vacuum-tight foil windows into the gas lasing volume; the primary electrons create secondary electrons in the $\mathrm{CO}_{2}$ gas mixture. When the high electric field from the main power source is applied to the preionized gas region, the secondary electrons are acceleraied and undergo inelastic collisions with the gas molecules. Through these collisions the gas molecules are raised to excited vibrational states (Fig. 3). As the pulse passes through the discharge region, it stimulates the emission of photons from the excited molecule. The molecular transition energy is transferred to the pulse, amplifying its energy. The amplification of the discharge medium is characterized by the quantity $\mathrm{e}^{\mathrm{gL}}$, where $\mathrm{g}$ is the net gain per unit length and $L$ represents the length of the gain region. In general, gL values are about $6-8$ in typical laser amplifiers.

The imfortance of the preionization of the laser gas is emphasized by the fact that the electric field strength in the discharge region required to create good secondary electron excitation results in voltage differences of about $500 \mathrm{kV}$ in regions of reasonable size. Without the preionization of the gas. catastrophic high-voltage arcs would form.

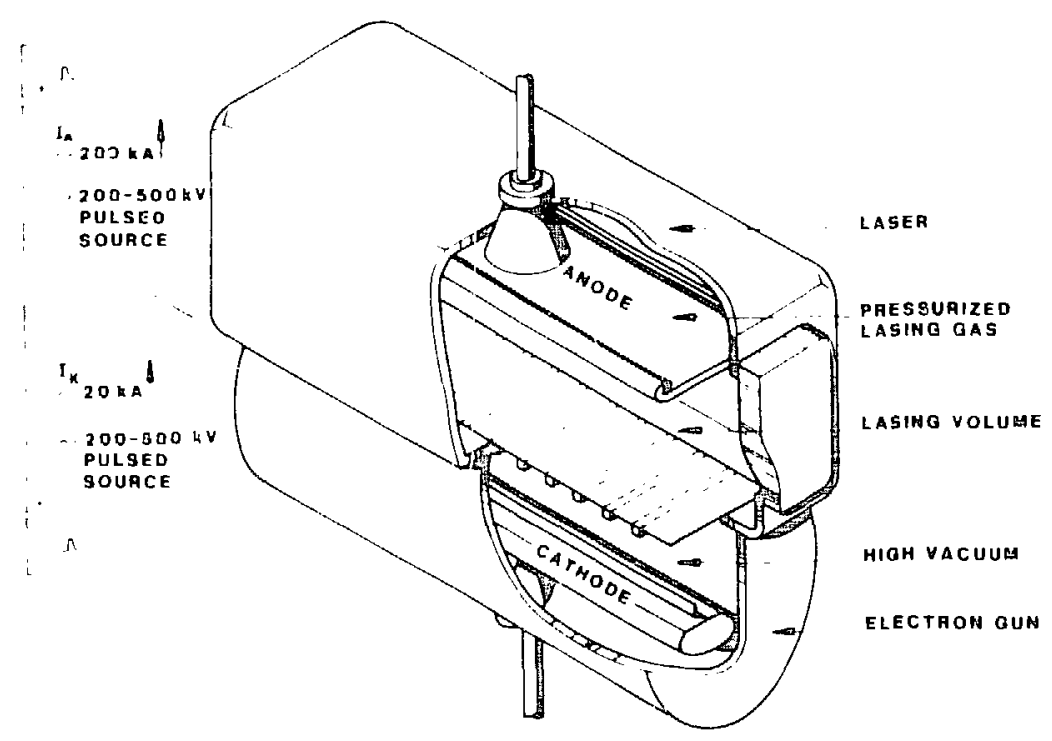

Fig. 2. Elements of an electron beam sustained gas discharge laser. The beam of electrons from the electron gun ionizes the laser gas. Fnergy can then be uniformly deposited in the gas by the main gas discharge. 


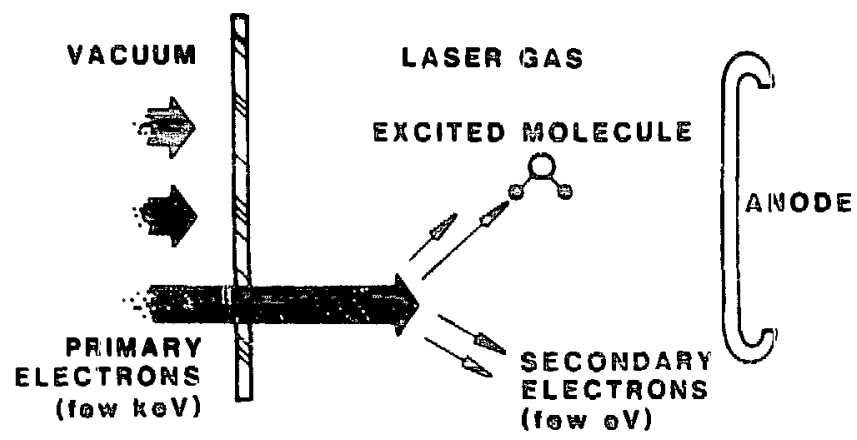

(a) ELECTRICAL EXCITATION

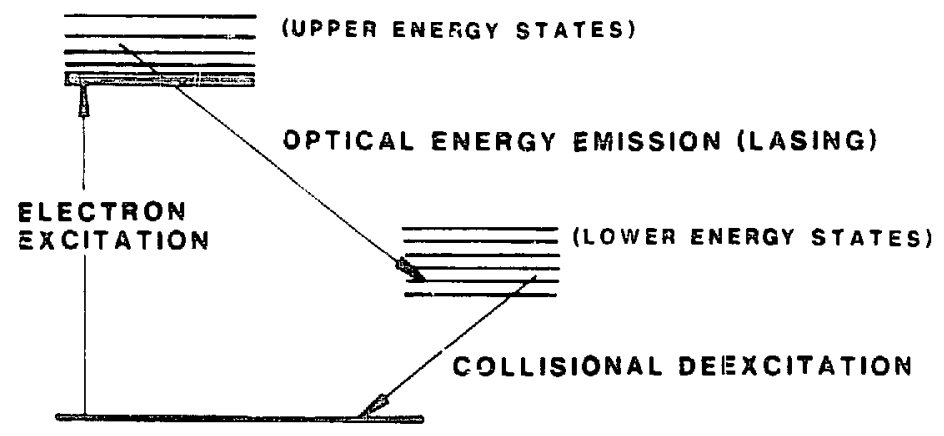

(b) LASING

Fig. 3. Dynamics of the laser process: (a) deposition of electrical energy in the laser gas by secondary electron collisions with gas moleicules; (b) extraction of the optical energy from the excited gas molelcules.

\section{ANTARES AMPLIFIER DESIGN}

The design of the Aitares power amplifiers is the result of evolution through the earlier designs and completed systems. The first system fabricated was similar to the one shown in Figs. 2 and 4a.

In this configuration, one electron gun served one amplifier The final single-power amplifier had an optical aperture of $25 \mathrm{~cm}$. In the interest of design economy and to reduce the number of pulsed-power systems required to drive the electron guns, the next generation system used one gun to serve two amplifiers as shown in Fig. 4b. The Gemini (2-beam) and Helios ( 8 -beam) laser systems successfully used this design of dual optical beam modules. The optical aperture of these systems was scaled up to $35-\mathrm{cm}$ diameter. Figure $4 \mathrm{c}$ indicates a pate $:$ ted but not fabricated design in which one gun served four amplifiers. The ultimate logical extension of thought then led us to use one gun to serve an annular laser ampinier as shown in Fig. 4d. This indeed represents schematically the
Antares power amplifier configuration; it is a 12 -sector annula- design with a $46-\mathrm{cm}$ optical aperture. The evolution of the design of power amplifiers at AVCO Everett Research Laboratory followed a similar path and resulted in the design of an annular system.

The original design goal of the Antares laser was to reach scientifi: breakeven with laser fusion. To this end Antares was designed as a six-amplifier system with an output of $100 \mathrm{~kJ}$ in a 1 -ns pulse. By 1980, sufficient questions had arisen from the experience with Helios and the advancement of computer models to give doubt as to whether this goal could be reached. These questions centered on the efficiency of the coupling of the $10-\mu \mathrm{m}$ laser light with the target. As a result the goal of Antares was changed tu one of evaluation of $\mathrm{CO}_{2}$ as a laser fusion driver by extrapolation of the Helios data to higher energy. With the new goal in mind, the Antares system was redesigned as a two-amplifier system that would be capable of delivering $40 \mathrm{~kJ}$ to a target in a $\mathrm{I}-\mathrm{ns}$ pulse. 


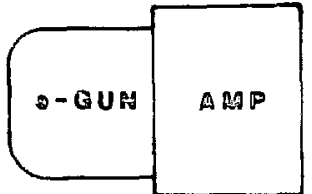

(a) SINGLE SYSTEA

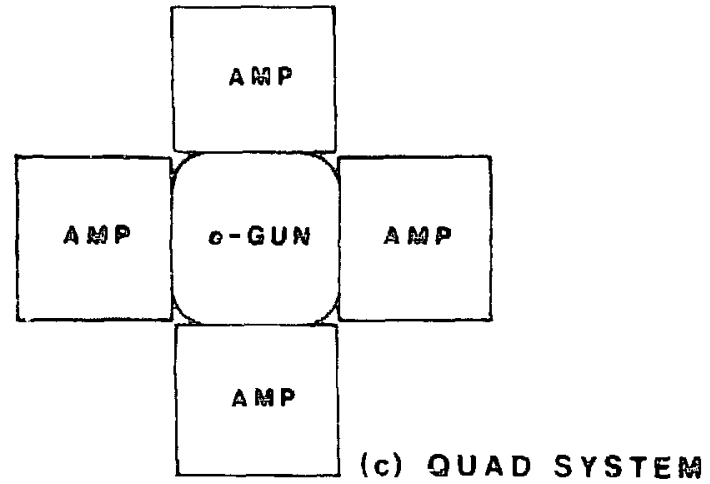

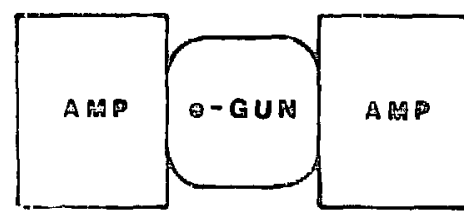

(b) DLAL SYSTEA (HELIOS)

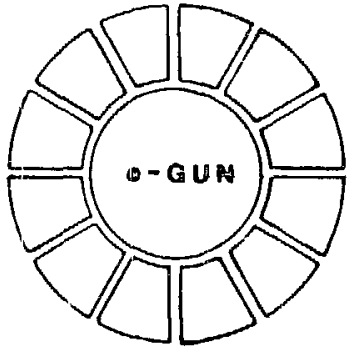

(d) 12 BEAA (ANTARES)

Fig. 4. Evolution of the design of high-energy gas discharge lasers. The sharing of one electron gun by seteral dis charge regions creates a considerable savings in complexity and cost.

The analytical efforts resulted in computer modeling codes that were refined as the early hardware systems came on line and as data from experimental operations became available. These refined codes provided the basis for the engineering design of the Antares laser system. These codes enabled us to predict the engineering parameters for any system corfigurations and included (1) selection of $\mathrm{CO}_{2}$ gas mixtures: (2) specification of discharge sizes, pressures. gas voltages, and currents: and (3) prediction of electric and magnetic effects or the discharge and gun current trajectories.

As shown in Fig. 4d. the general design of the Antares power amplifiers consisted of 12 discharge regions spaced in an annulus around a central electron gun. This design was further refined to have four discharge regions spaced uniformly along the length of the amplifier. The final result was a system with 48 discharge regions of 33 . $\mathrm{cm}$ thickness, a $35.7 \mathrm{~cm}$ trapezoidal half-width, and a 75 $\mathrm{cm}$ length. This annular design represented a compac packaging concept and was a radical departure from the configurations of previous laser systems.

Before the Antares design. the lasing mixes consisted of $\mathrm{CO}_{2}, \mathrm{~N}_{2}$, and $\mathrm{He}$. One reason helium was used was that it permits the desired electron energies to occur at lower electric fields in the discharge, thereby reducing the chance of high-voltage potential arcing. In a system as large as Antares. helium recovery is botk; necessary and expensive. Calculations indicated that higher energy could be stored in He-free mixtures, which would result in higher energy extraction. The use of He-free gas mixtures would result in a more efficient laser amplifier. However. high-pressure. He-free electric discharges required higher voltages $(\sim 500 \mathrm{kV})$ than had been used previously. It was r.ot known if high-voltage cables and terminations were practical under these operaing conditions. Substantial magnetic effects were predicted on the discharge uniformity. Parasitic oscillations both linear and circumferential in the high-gain amplifiers were of major concern and not amenable to sufficiently reliable calculations.

\section{SYSTEM PROTOTYPES}

The technical uncertainties prompted the constiuction of a prototype amplifier that could reasonably duplicate power amplifie: sizes and problems. The prototype design was full scale in diameter. The gain region was longer $(150 \mathrm{~cm}$ vs $75 \mathrm{~cm})$ than the iength of each of the four gain 
subsections in the amyitiier design. The longer gain length provided a rigid test of magnetic field effects in that they were more severe than those in the actual amplifier designs. Only 3 of the 12 prototype discharge channels were electron-beam-sustained discharge channels. In the other nine channels, the discharge was simulated by the use of conductors.

The prototype amplifier answered many of the uncertainties in the Antares design:

- A new design of an efficient electron gun was demonstrated. This electron gun incorporated a selfbiasing grid for current regulation.

- High-voltage. Helium-free operation proved to be successful.

- A prototype induct?nce-capacitance energy storage network worked well.

- Cables and cable terminations designed for 500-k. V operation performed as expected.

- Satisfactory gain distribution across the discharge as well as gain shaping in the individual channels was demonstrated.

- Parasitic oscillation thresholds were demonstrated to be manageable.

The prototype program demonstraced that adequate performance could be expected from the Antares power amplifiers. The major physics and engineering uncertainties in the design were rescived and the program could proceed on schedule.

There were other prototype programs in progress at the same timc as the power amplifier prototype. These prototypes included beam diagnostics and beam alignment. The beam alignment prototype was a major undertaking in itself. This prototype consisted of a layout of a single sector of the optical train incidting computer control of all of the necessary elements. The later prototyping proceeded in parallel with the fabrication of the power amplifier. Careful planning of the experimental programs was required to bring these later prototyping efforts to the hardware stage in time to fit into the construction schedule.

In the prototype program, we developed antireflecting surfaces, which were optically black at $\lambda=10 \mu \mathrm{m}$, that proved effective in parasitic suppression. These were based on LiF, an excellent absorber of $\mathrm{i} 0$ - $\mu \mathrm{m}$ radiation. Antireflecting surfaces were made by LiF plasma spraying or by the application of LiF-loaded paint. The prototype itself yielded data that wore used to further refine the computer codes necessary to improve the Laboratory's modeling capability.

\section{FINAL ANTARES CONFIGURATION}

The present Antares laser system (Figs. 5 and 6) is combined result of earlier experience with the th previous laser systems and the extensive prototype $t$ ing.

In the Antares system, the front end must delive 100-J, I-ns pulse, to each of the main power amplif with the proper combination of energy, $\mathrm{CO}_{2}$ line cont contrast ratio, and pulse shape. The front end is oscillator-preamplifier chain as indicated in Fig. 1. 7 short-pulse generators (Fig. 7) are currently in plac. the Antares system: one from the Gemini laser syst which represents reliable, "old" te "hnology, and a sec that is just becoming operatiorial a. 'd represents state the-art development. The new shor $($-pulse genera:or permit long-pulse (to 10-ns) shaping currenty require inertial confirement fusion experiments. This oscill: syster: is shown in Fig. 8. It consists of three indepe ent. single-line $\mathrm{cw}$ oscillators that are grating tunec operate on a specific wavelength. The amplitude pulse length of each independent oscillator output $p$ can be selected. The independent pulses are combi and amplified in the first preamplifier stage. An elec optic switch (a Pockel cell) is used to switch out the f combined pulse length. The switch provides the propriate energy contrast ratio.

The pulse is divided into two by a bearn splitter. beam for each main power amplifier, before the $f$ stages of preamplification. The final pieamplifier stag called the driver amplifier. This amplifier (Fig. 9 patterned after the Helios amplifiers (Fig. 4b) with exception that the driver amplifier electron guns are $g$ controlled devices. The driver amplifier electron $g$ produce two beams of $250-\mathrm{kV}$ electrons with a cun density of $50 \mathrm{~mA} / \mathrm{cm}^{2}$. The amplifiers operate with a mix of $\mathrm{N}_{2}: \mathrm{CO}_{2}$ at a pressure of 1200 torr. The po source for the discharge is a Marx generator. wh develops a voltage pulse with a peak voltage of 300 The optical train in the driver amplifiers is configured three-pass, on-aris, Cassegranian system. The $1-J$ in to the driver amplifiers is amplified to $100 \mathrm{~J}$ and sen the power amplifiers as an annular beam with an or diameter of $15 \mathrm{~cm}$ and an inner diameter of $9 \mathrm{~cm}$.

The power amplifiers must accept the 100-J out from the driver amplifier and raise the pulse energy to $15-$ to $20-\mathrm{kJ}$ design level at its exit. The final des configuration is shown in Fir. 5. 


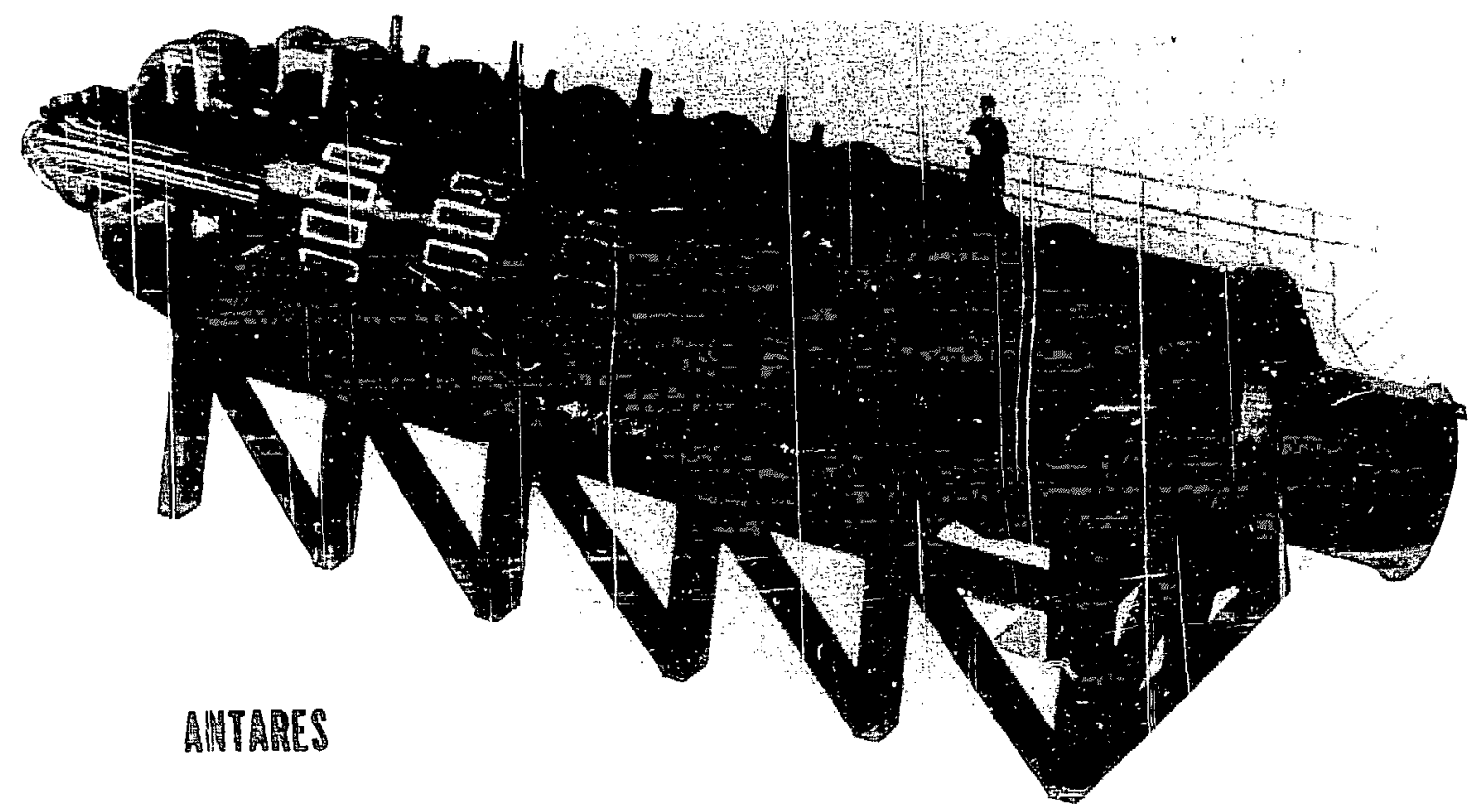

\section{POWER AMPLIFIER}

The general configuration of the power amplifiers is of 12 active sectors in an annular array around a central electron gun. The dimensions of the amplifier are in turn controlled by the necessity of keeping the laser energy fluences below the damage threshold for optical materials. In the case of mirrors, absorbed energy from the beam can cause surface melting if the incident energy flux is too high. The high electric fields associated with the short. powerful ligint pulses cause dielectric breakdown in transmissive elements when the light flux is above damage threshold. The best transmitter of $10-\mu \mathrm{m}$ radiation is $\mathrm{NaCl}$. The only windows in the system are needed to frorm the boundary between the target vacuum and ambient air and between the target vacuum and the lasing gas. The state of the art in fabrication of $\mathrm{NaCl}$ along with the damage threshold and the required output in turn determine. respestively, the total output area, the size of the individual sectors, and ultimately, the number of sectors. For polycrystalline $\mathrm{NaCl}$, an 18 -in. diameter is the state of the art. Allowing for optical mounting, $1000 \mathrm{~cm}^{2}$ is available in each sector ther availabie with 12 sectors for beam passage. A reasonable average energy flux is $1.7 \mathrm{~J} / \mathrm{cm}^{2}$. Each of the 12 sectors can deliver $1700 \mathrm{~J}$ or a total of $20.4 \mathrm{~kJ}$ for a power amplifier. The copper-surfaced mirrors are designed for fluxes of 4 $\mathrm{J} / \mathrm{cm}^{2}$

\section{POWER AMPLIFIER ELECTRICAL PARAMETERS}

The size of the optical windows determines the size of the individual discharge regions. To keep within those limits and provide a reasonable discharge region from the standpoint of the electrical requirements, the discharge regions were designed with a nearly trapezoidal cross section as is shown in Fig. 10. From the data obtained during work with the prototype amplifier and the results of the computer modeling, the laser mix was cnosen as $4: 1 \mathrm{~N}_{2}: \mathrm{CO}_{2}$. At a pressure of 1800 torr and with four gain regions of $75-\mathrm{cm}$ length, iasing conditions with sufficient energy storage is reached with a specific electric field of $10 \mathrm{~V} / \mathrm{cm}$-torr and a current density of $7 \mathrm{~A} / \mathrm{cm}^{2}$. These requirements in turn demand that the anode voltages be nearly $550 \mathrm{kV}$ and the discharge impedance be around 2.5 ohms. 


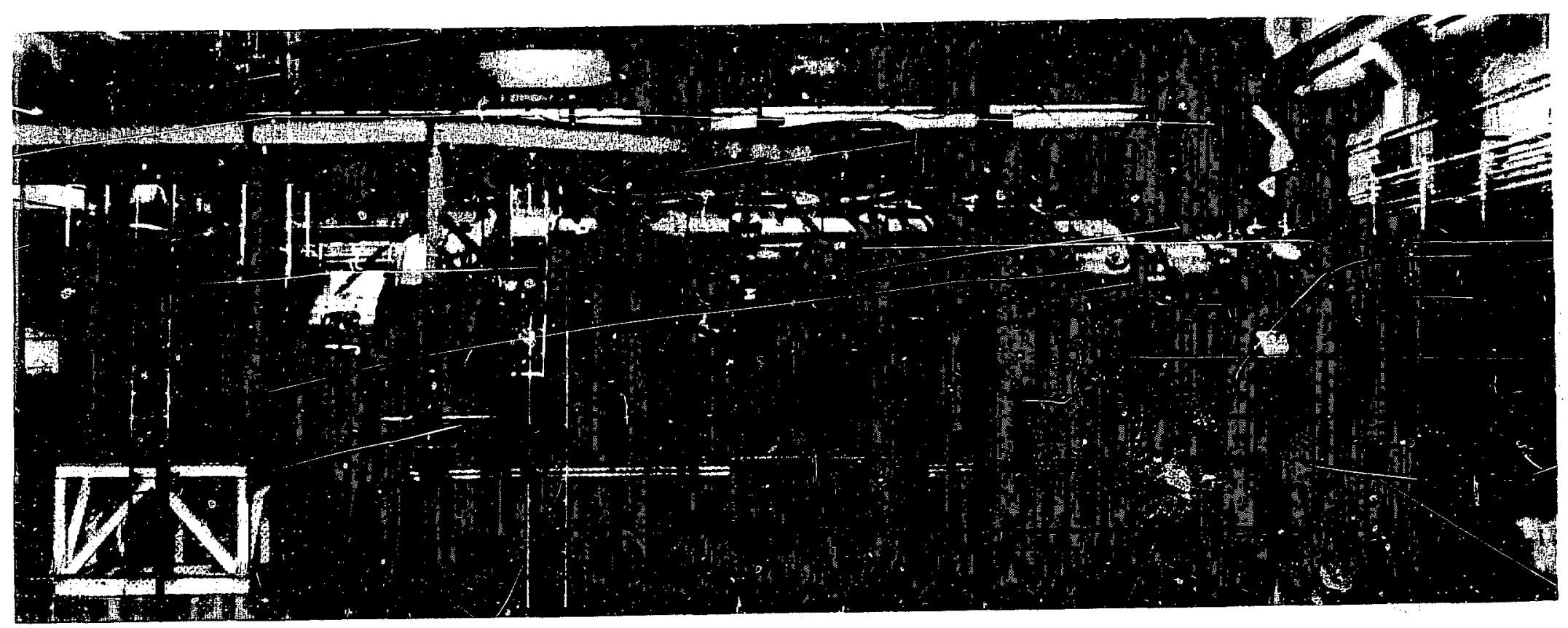

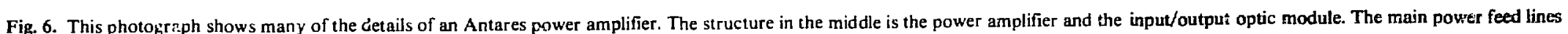
can be seen to enter the power amplifier in fous regions. The electron gun Marx, the structure on the left, is the power supply for the electron gun. 


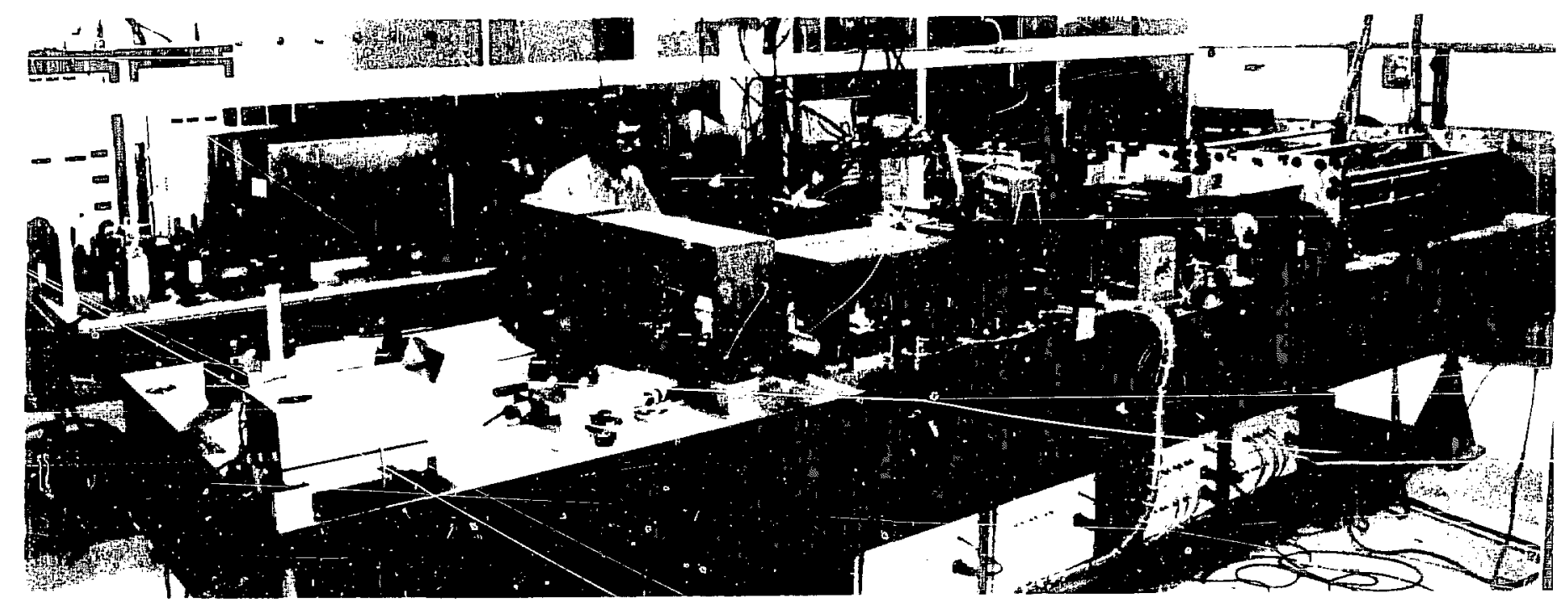

Fig. 7. The multiline oscillator (foreground) and the Gemini oscillator (background) are located in the oscillator room of the Antares front end 


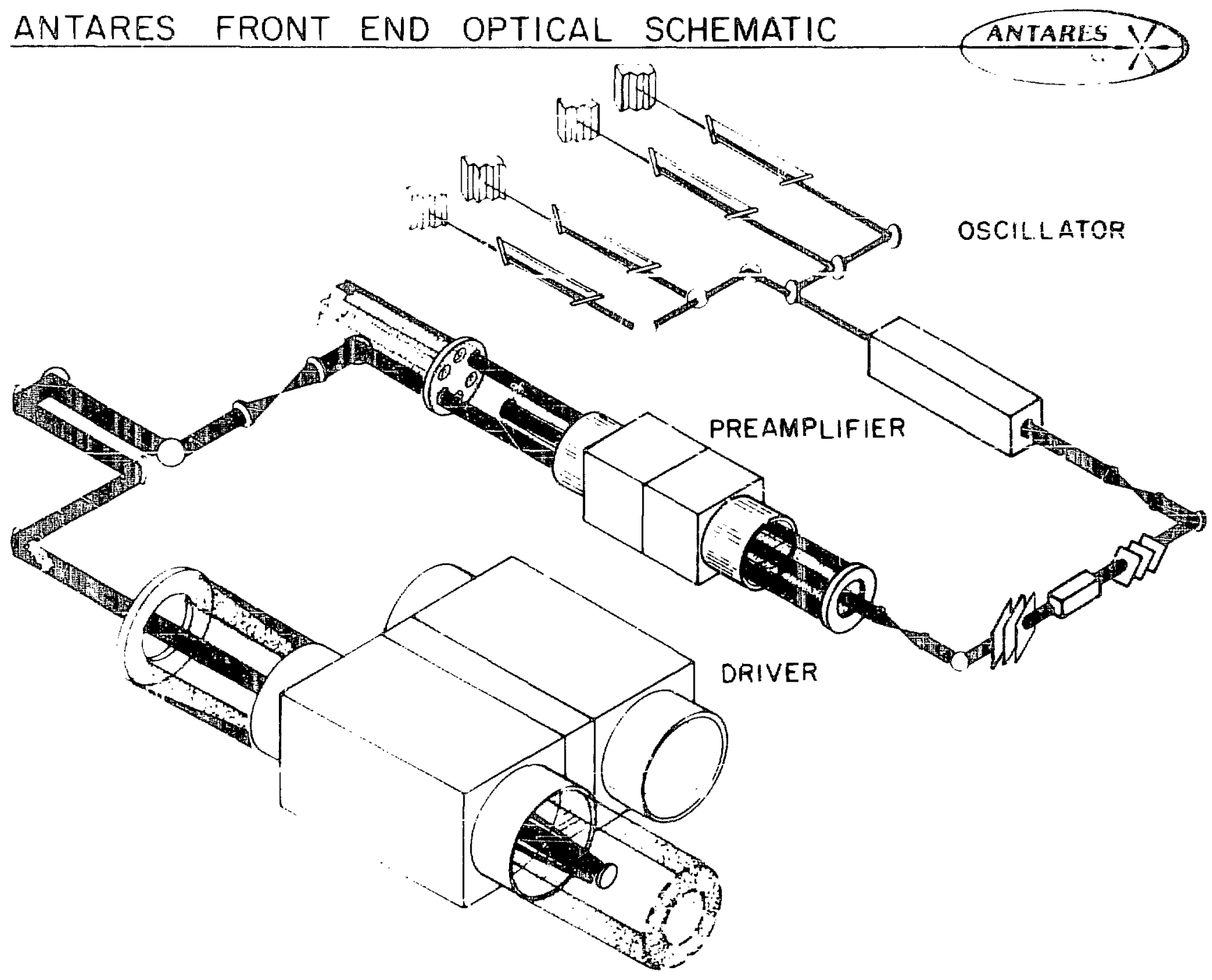

Fig. 8. Optical schematic of the Antares f: ont end. The pulses that are amplified by the power amplifiers are formed in the front end. A short pulse is generated in the oscillator and then amplified to be a 100-J. 1-ns pulse after two stages of amplification. 

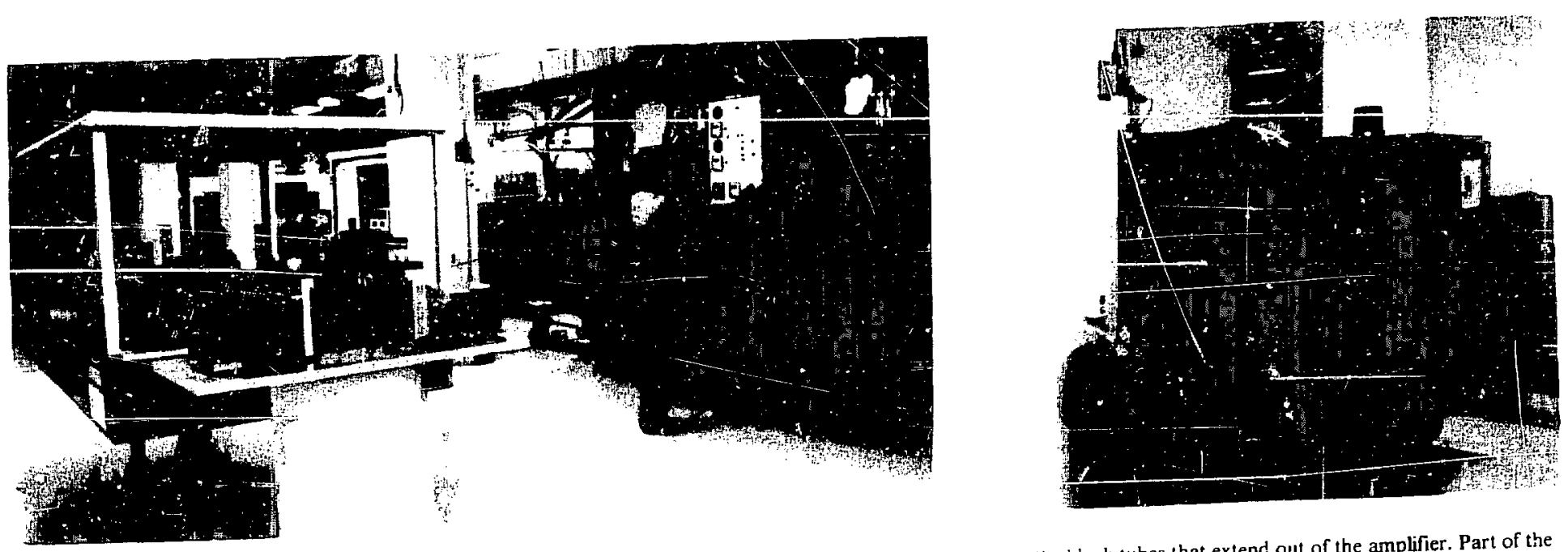

r.- o Tho drivar amrilfier is located in the amplifier room beam forming optics are located on the table at the left. 


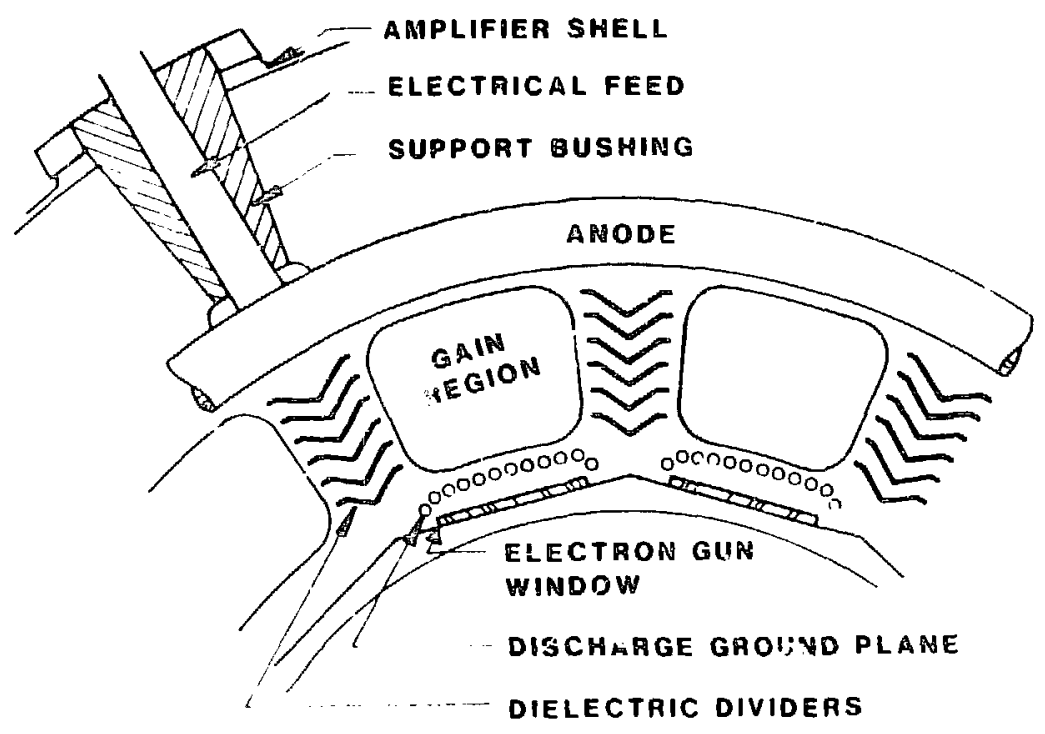

Fig. 10. Cross section of a portion of the gain region in the Antares power amplifier. Twelve independent gain regions are electrically and optically separated by dielectric (nonconductive) divilers. Each group of 12 repions is electrically driven from one anode. Six electrical feed lines supply each anods.

The time constant of the relaxation of the upper $\mathrm{CO}_{2}$ laser level of $4.5 \mu \mathrm{s}$ makes short pump times more efficient. A iaser pumping time to peak gain of $3 \mu$ s was selected as a compromise between desirable high pumping efficiency (short pulse), low current density (long pulse), and large circuit inductance (long pulse). Lower current densities produce lower magnetic fields and, thereby, less undesirable electron beam deflection. Large circuit inductances aie easier and less costly to achieve. The characteristics of the Marx generators, which power the gas discharges and the electron gun, are shown in Table 1 . The design gain of he laser is $2.7 \mathrm{~m}^{-1}$; hence, the amplifiers were designed to achieve a single-pass gain of 8.0. The physical size of the Antares power amplifiers and their relationship in the laser hall can be secn ir. Fig. 11.

\section{POWER AMPLIFIER OPTICAL SYSTEM}

The optical layout of the Antares system is shown in Fig. 12. This figure gives the relationship between the various segments of the optical system. The power amplifier optical system is designed as a two-pass, offaxis Cassegranian system. A single sector of the power amplifier optical train is shown in Fig. 13. The input 100-J, annular beam is subdivided into 12 segments by a machined polygonal copper mirror. The input segments

\begin{tabular}{|c|c|c|c|c|c|c|}
\hline & $V_{0}(k V)$ & $V_{L}(k V)$ & $\mathbf{I}(\mathrm{kA})$ & $Q(C)$ & $\mathrm{L}(\mu \mathrm{H})$ & $\mathrm{C}(\mu \mathrm{F})$ \\
\hline Power Arnplifier & $1000 / 12000$ & $450 / 550$ & $150 / 200$ & $\sim 1.0$ & $\sim 2.4$ & 0.420 \\
\hline Electron Gun Marx & $525 / 600$ & $475 / 550$ & $20 / 25$ & $\sim 0.1$ & $\sim 3.5$ & 0.375 \\
\hline \multicolumn{2}{|c|}{$\begin{aligned}{ }^{a} V_{0} & =\text { Open circuit voltage; } \\
V_{L} & =\text { Peak load voltage; } \\
& =\text { Peak load current }\end{aligned}$} & \multicolumn{5}{|c|}{$\begin{array}{l}Q=\text { Charge transfer } \\
L=\text { Marx inductance } \\
\text { C = Marx capacitance. }\end{array}$} \\
\hline
\end{tabular}




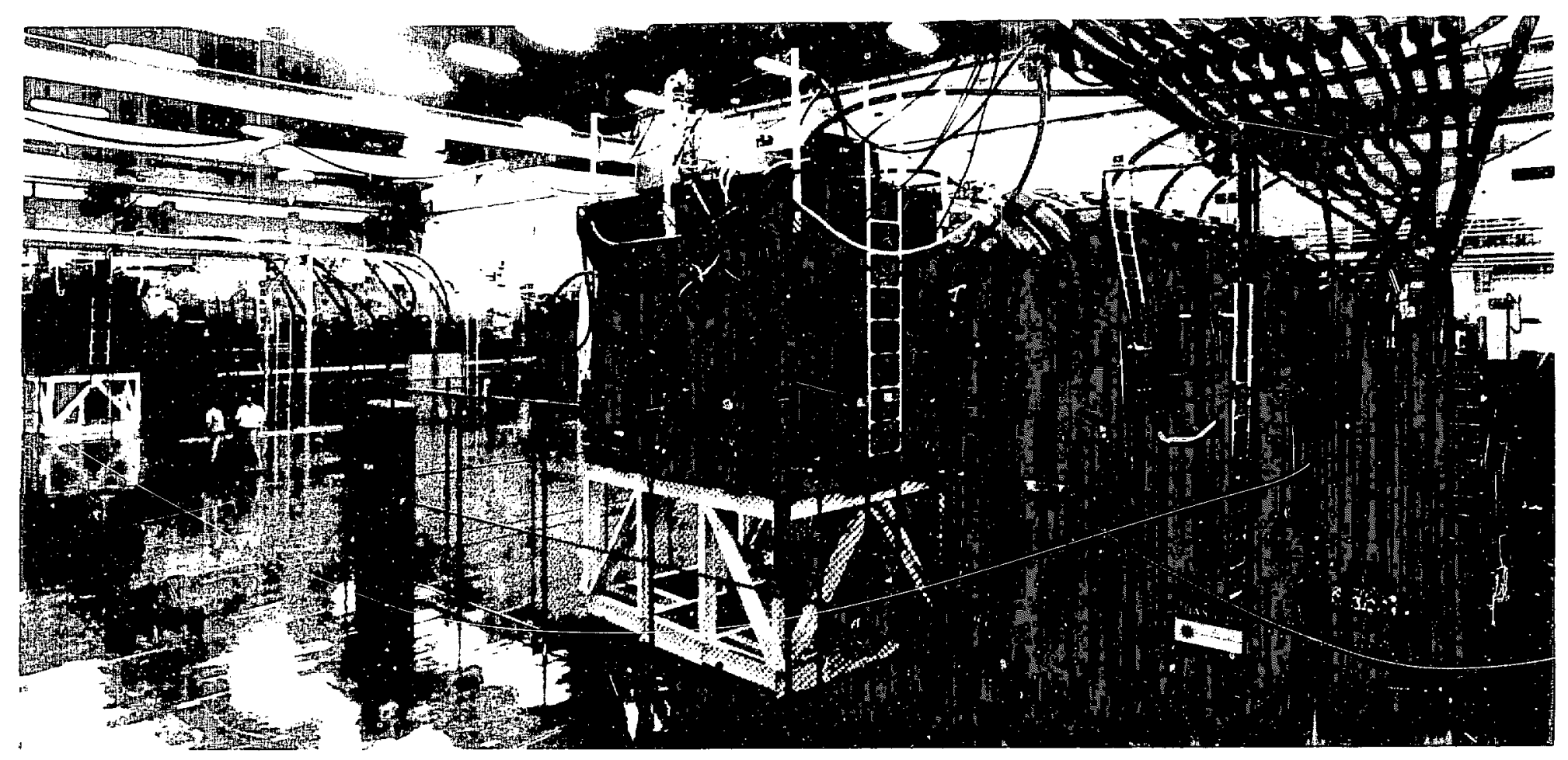

Fig. 11. The Antares Laser Hall. The room containing the two power amplifiers is nearly th size of a football field. 


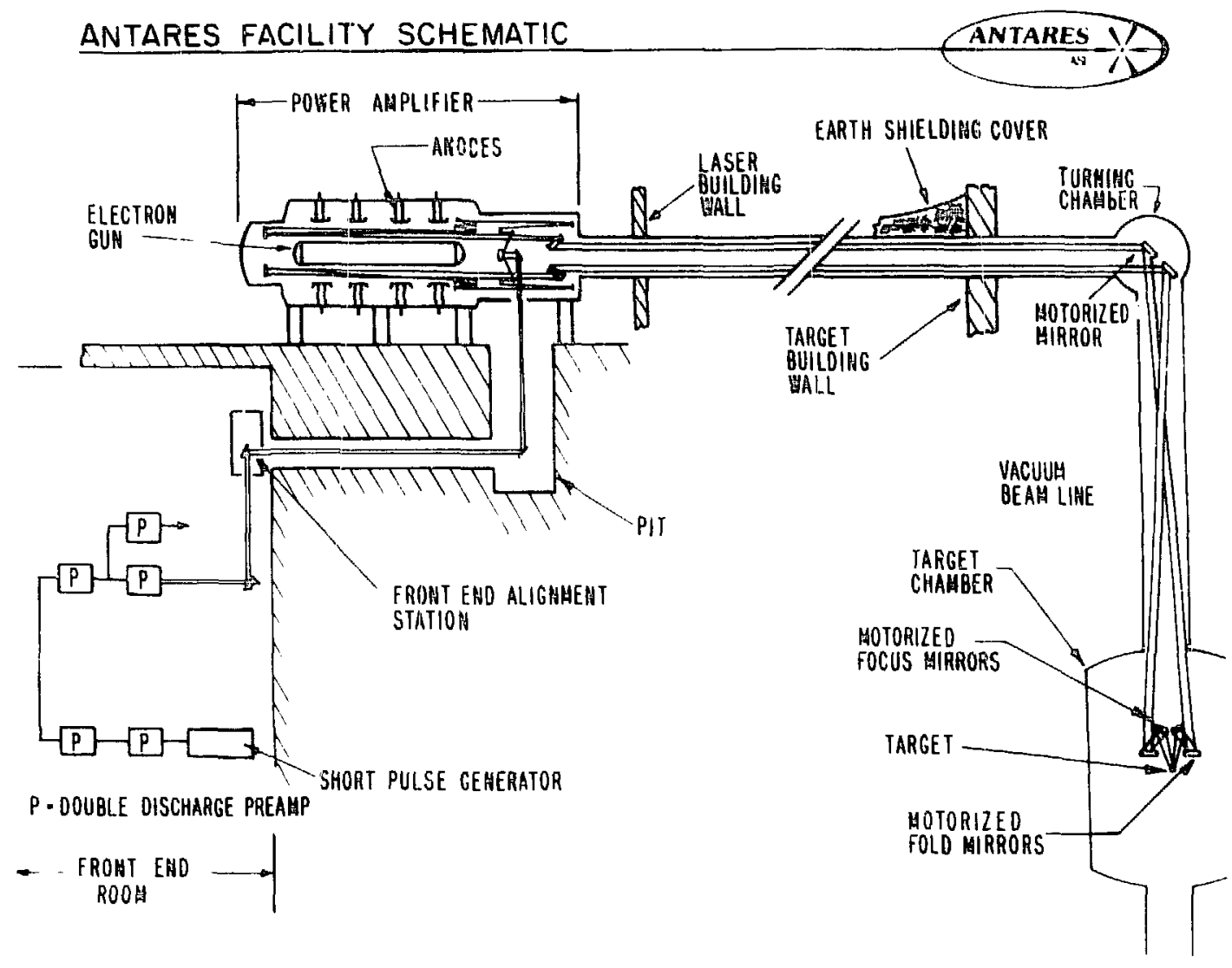

Fig. 12. The Antares optical system is laid out in four major segments. These segments are the front end, the front end alignment station. the power amplifier. and the target system.

have an area of about $10 \mathrm{~cm}^{2}$. Each of the 12 sector beams is brought to a focus in a spatial filter fabricated from LiF. The plasma generated at the focus lasts for several microseconds and serves as an optical shutter to prevent the return of back-running beams to the front end where with further amplification they could do major damage. The pulse is then introduced into the power amplifier as a divergent beam. After its first pass through the four gain regions, it is reflected and collimated by a concave mirror for a second pass through the discharge region. The 5-J input energy insures that as the pulse makes its second pass through the discharge, virtually all of the available energy stored in the amplifiers is extracted. As the 12 beams exit the power amplifier, they are compressed into an annulus of $1-\mathrm{m}^{2}$ area and an outer diameter of nearly $1.5 \mathrm{~m}$.

\section{PARASITIC OSCILLATION SUPPRESSION}

In a high-gain cavity, as exists in the Antares systen the suppression of parasitic oscillations is of critici importance. Nonreflecting, optically black surfaces ( $c$ $10 \mu \mathrm{m})$ are re(guired. During the early stages of testing, a of the exposed surfaces within $5 \mathrm{~cm}$ of the beams wer coated with a paint loaded with LiF. Even though thi paint had a very low reflectivity, oscillations were er countered when the amplifier was pumped at a single pass gain greater than about 2.7. A great number $c$ possible parasitic modes were identified, some of whic were characteristic to the Antares mechanical desigr One of these was a ring laser mode in which a ray fror the amplifier collimating mirror could strike an off-axi surface after passing through the four gain iegion: 


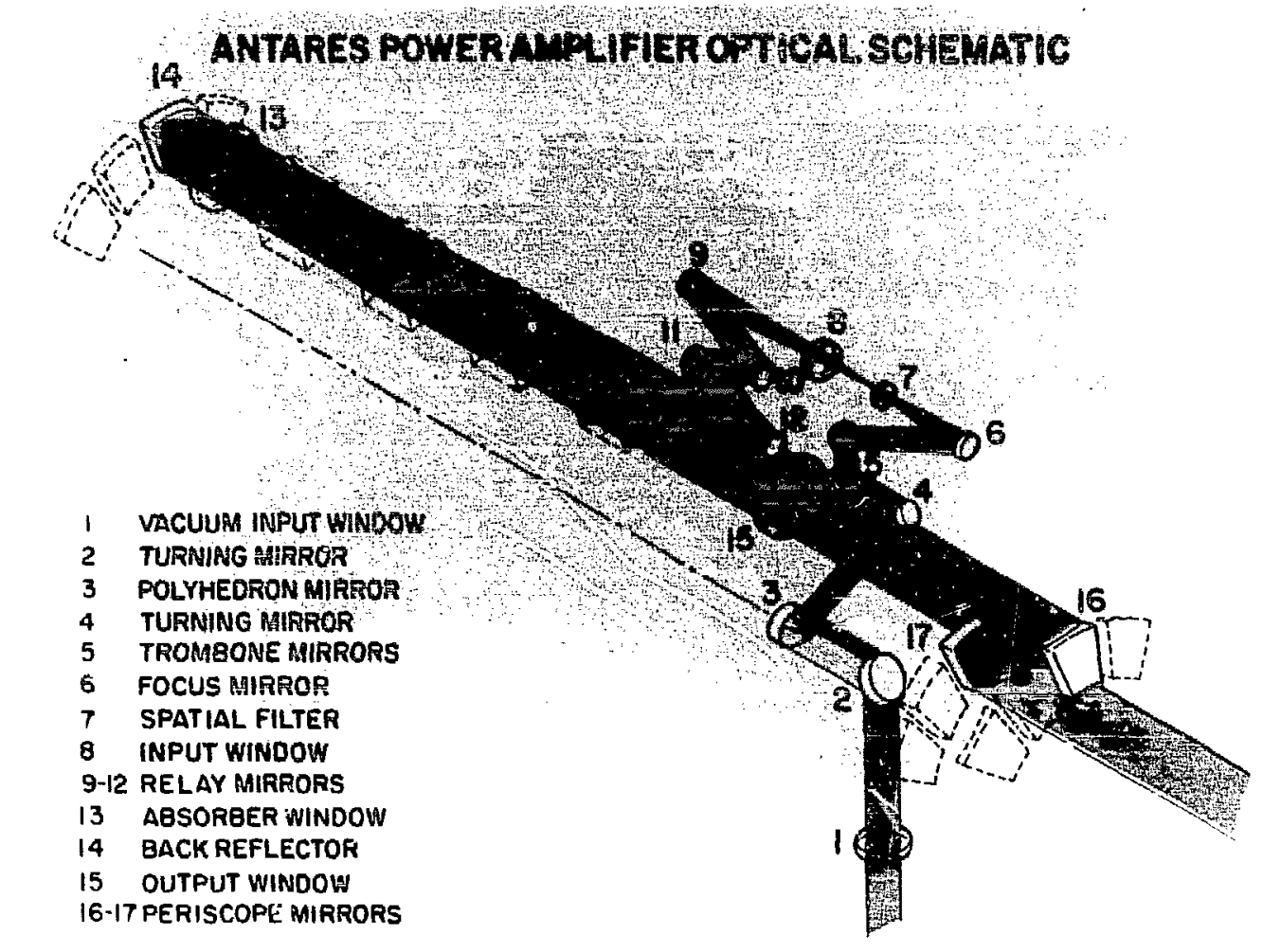

Fig. 13. One sector of the power amplifier optical train. The cross-sectional area of the optical beam increases in proportion to the energy so that the energy density remains below the damage threshold for optical materials.

undergo a Fresnel reflection of the output window, and return to the collinating mirror through the four gain regions. It. was necessary to design a set of baffles coated with plasma-sprayed $\mathrm{LiF}$. After installation of these baffles. the stability threshold of the entire system rose to a net gain of 6.0 . The addition of saturable absorber to raise the stability limit to a net gain of 8.0 provides a very small loss in net amplifier output. As a result of the use of plasma sprayed $\mathrm{LiF}$ baffles, the Antares laser is one of the. if not the most, stable high-power lasers ever built.

\section{TARGET SYSTEM}

The target system contains both the target vacuum sysiem and the target optical system.

\section{Target Vacuum System}

The beams from both of the power amplifiers travel through a vacuum from the output of the power amplifier to the target. The system (Fig. 14) is made of stainless steel, enclosing a volume of about $1000 \mathrm{~m}^{3}$, which can be maintained at a pressure of $2 \times 10^{-5}$ torr. The pumping time to reach this pressure, about $8 \mathrm{~h}$, is limited by the possibility of damaging target diagnostic equipment. The power amplifiers are connected to the target chamber by $1.5-\mathrm{m}$-diam beam tubes. The target chamber is an $8-\mathrm{m}$ diam cylinder closed on both ends with spherical caps to produce a 7 -m-long volume. The vacuum is maintained with cryogenic pumps. Two pumps are mounted on the target chamber and two pumps are mounted on each of the beam tubes. Targets are inserted into the target 


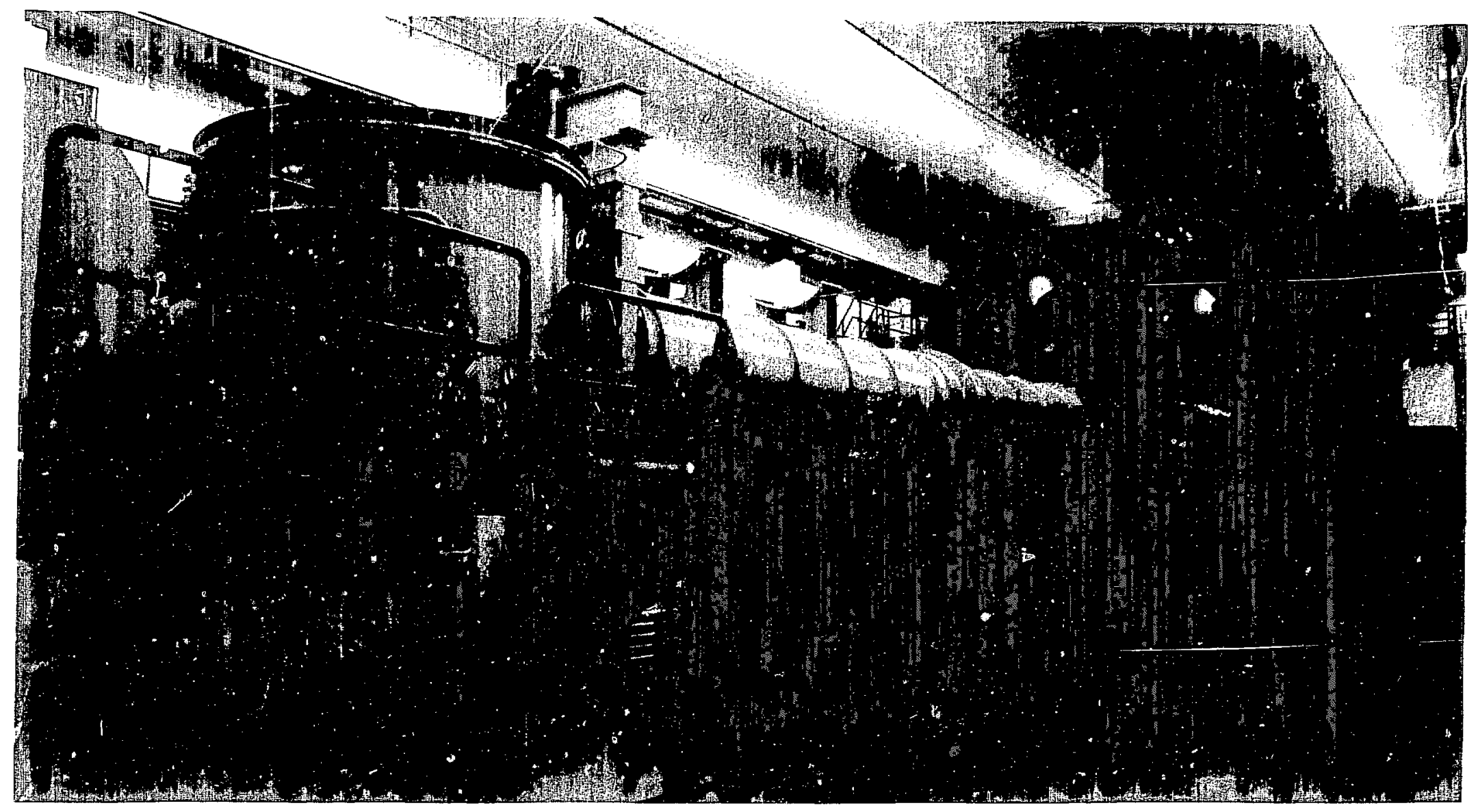

Fig. 14. The target vacuum system includes the beam tubes and the target chamber. Only one of the beam tubes leading from the power amplifiers is visible in this photograph. The target vacuum system encloses a volume of about $1000 \mathrm{~m}^{3}$ 
chamber through an air lock and transported on an inclined track to their central position. Final positioning of a target is performed by the use of two iarge reference iclescopes with a resolution of $10 \mu \mathrm{m}$.

\section{Target Optical System}

The 12 bearns are compressed into an annulus with a $1.5 \mathrm{~m}$ major diameter as they enter the target vacuum system. The individual beams are set to be slightly convergent as they leave the power amplifier. The beams would be brought to a focus at a point about $800 \mathrm{ft}$ from the power amplifier if there were no further focusing. At the turn chambers, the beams are grouped in to three clusters of four beams by directing them toward the fold mirrors in the target chamber. The positioning of the fold mirrors in the target chamber can be seen in Fig. 15. The three sets of fold mirrors direct the beams outward toward the 24 focusing mirrors. The focusing mirrors are diamond-machined, off-axis parabolas that operate at an $f$-number of 6.3 . The net result is that a target is illuminated on six sides by six beam clusters of four beams each.

A pointing accuracy of $25 \mu \mathrm{m}$ is requirer! at the target position for each of the 24 beams. At the request of an experimenter, the beams may be independently directed to any puint within a $1-\mathrm{cm}$ volume. The focal spot size is diffraction limited by the polyhedron mirror to $200 \mu \mathrm{m}$.

\section{ALIGNMENT SYSTEM}

Seismic and thermally induced movement generated by the impulse loading in the gas required an alignment system to bring the 24 beams to the proper pointing and centering on the optical beam paths. The optical alignment systern was chosen after experimental consideration of several approaches. The present "see-through" system was fully prototyped with actual hardware and computer controls before the decision was made to implement it. The alignment system is designed to automaticallv align if 8 optical elements.

Alignment of the optical system is carried out in two stages. First, the system is aligned with yisible light. Second. the fold and focus mirrors are adjusted with a cw $\mathrm{CO}_{2}$ laser

The visible alignment system relies on a video tracking system to identify light sources. The error signal from the tracker that characterizes the difference beiween the measured and the expected positions of the light sources is fed into a computer-controlled feedback loop that generates the signals to control the stepper motors on the appropriate mirrors. A typical feedback loop is shown in Fig. 16. The light sources are fiber-optic elements, which in some cases are imbedded in mirrors and in other cases are placed into position by movable arms. Secondary alignment is achieved by projecting a $\mathrm{CO}_{2}$ laser beam througn ine entire optical train and sensing its position at the target. Tnis correction eliminates dispersion errors between visible and $\mathrm{CO}_{2}$ wavelengths that arise chiefly from the wedged exit salt windows. The system has demonstrated alignment accuracies at the target of about $40 \mu \mathrm{m}$.

\section{BEAM DIAGNOSTICS}

The large apertures and high energy densities of the optical pulse required that new approaches be taken in diagnosing the quality of the Antares optical beams. New calorimetric techniques were developed to meet these needs. The energy transmitted at several points in the system is measured to provide an indication of the operation of the system on each shot. The following data are measured on each shot:

o total energy exiting the front end in each beam line.

o total energy entering the input salt window of the power amplifier,

- total energy entering each sector of the power amplifier,

- total energy encering the target system on each beam line, and

0 wave shape of the total pulse entering the target system from each beam line.

Since the measurements of amplifier energy must be made on a noninterference basis, a means had to be devised to sample a fraction of the beam energy. The :neasurement of the output of the power amplifiers presented a particular problem in that the space required to sample all of the sectors would have been prohibitive. To overcome this difficulty, the decision was made to sample the integrated output of the entire amplifier. This was accomplished by designing the output salt windows with a wedge angle between the surfaces and arranging the positions of the windows so that the second Fresnel reflection from each window produced a beam that was directed toward the center of the beam tube. The configuration : shown in Fig. 17. By proper choice of the wedge angle of the windows, the 12 sector beams were 


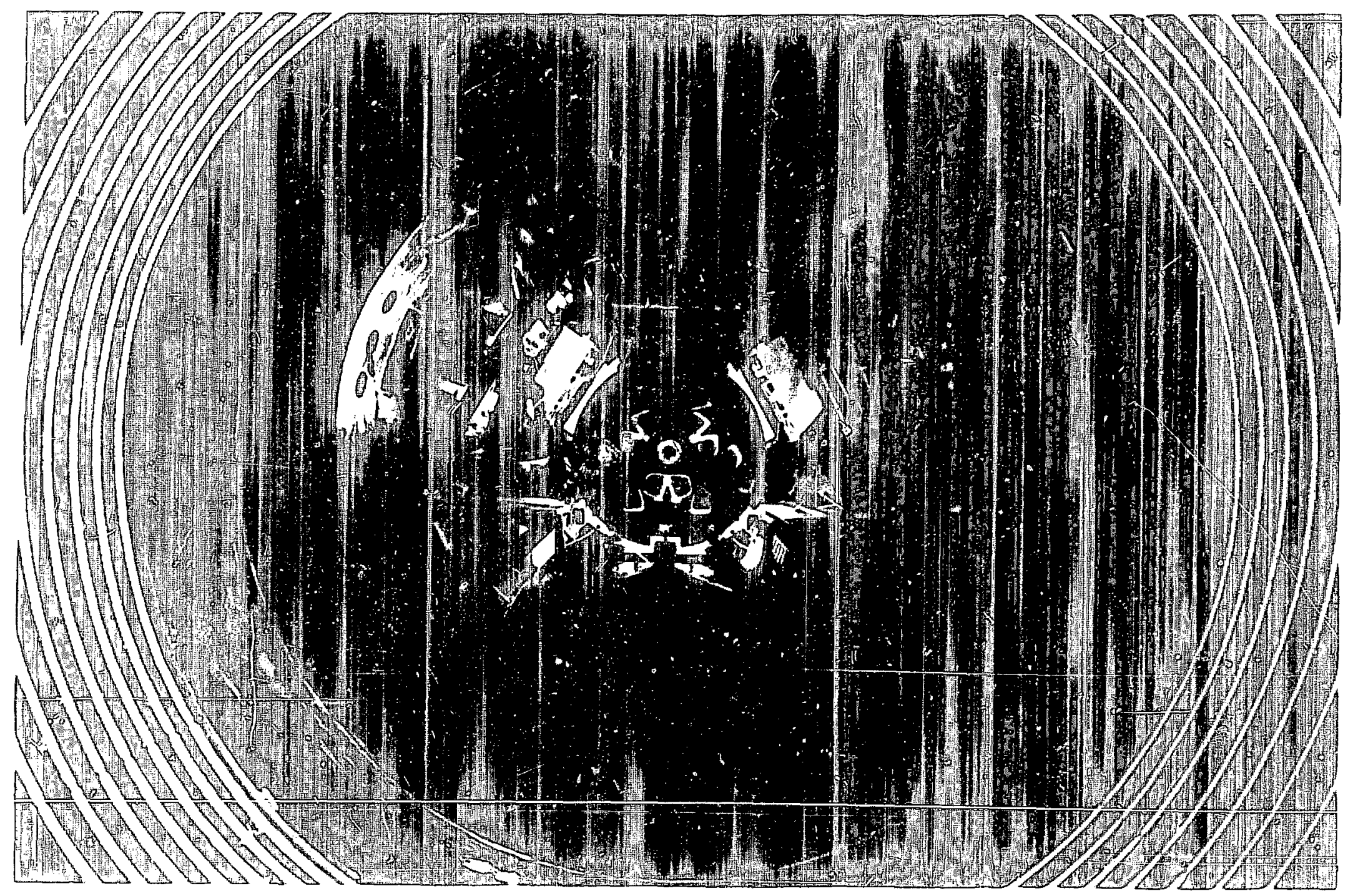

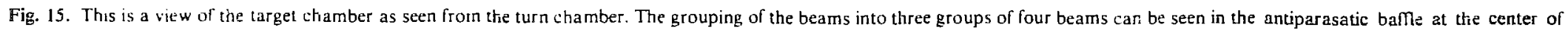
the photograph. The targets are mounted within the structure a: the center of the scene. 


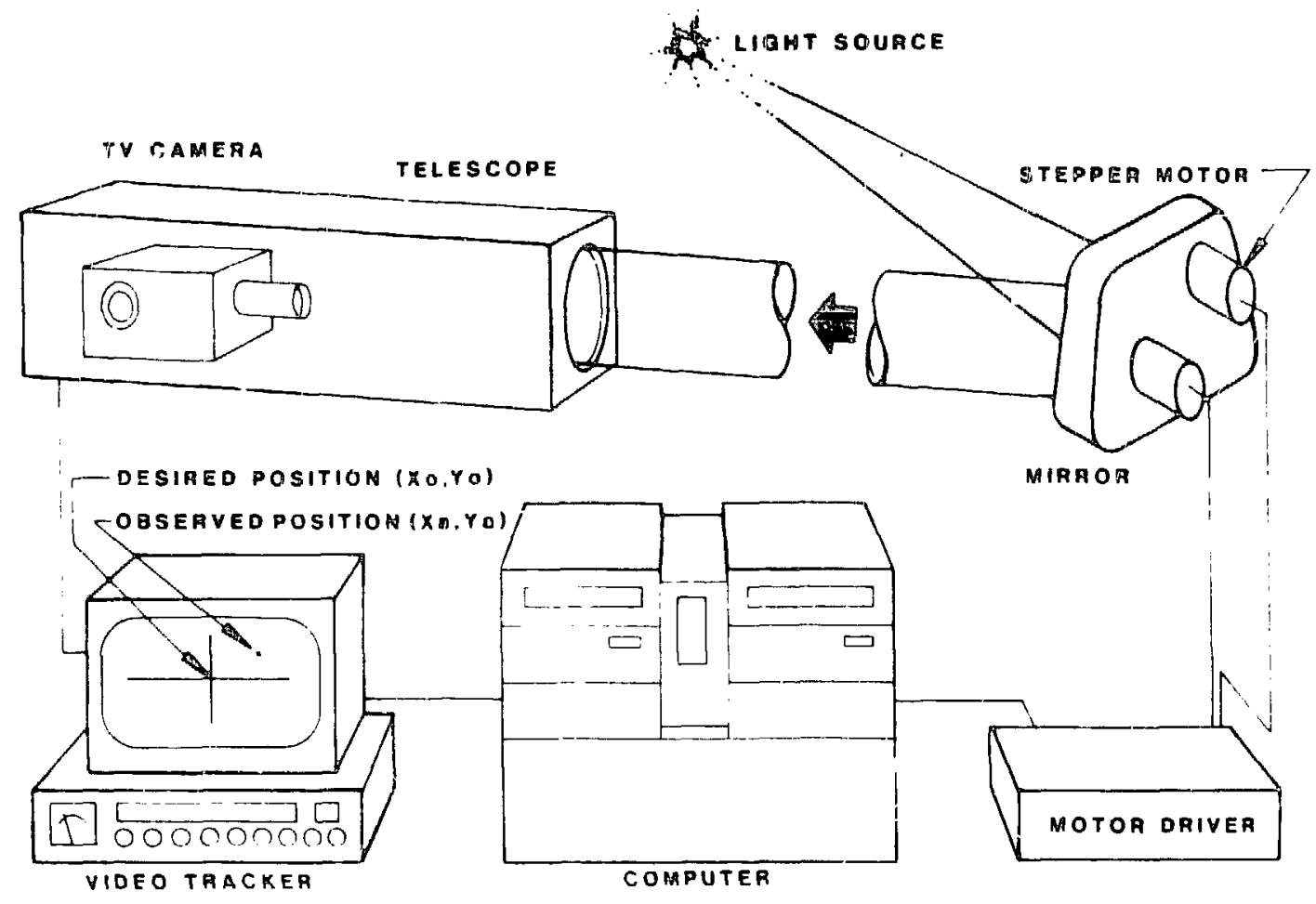

Fig. 16. Alignment of the optical train is carried out in several stages by a computer controlled fee." Jack loop. Visible light sources at entical locations are centered in the field of view of a TV camera by feeding the alignment error to a computer that in iurn dnves the stepper motors on a mirror in the correct direction to minimize the alignment error.

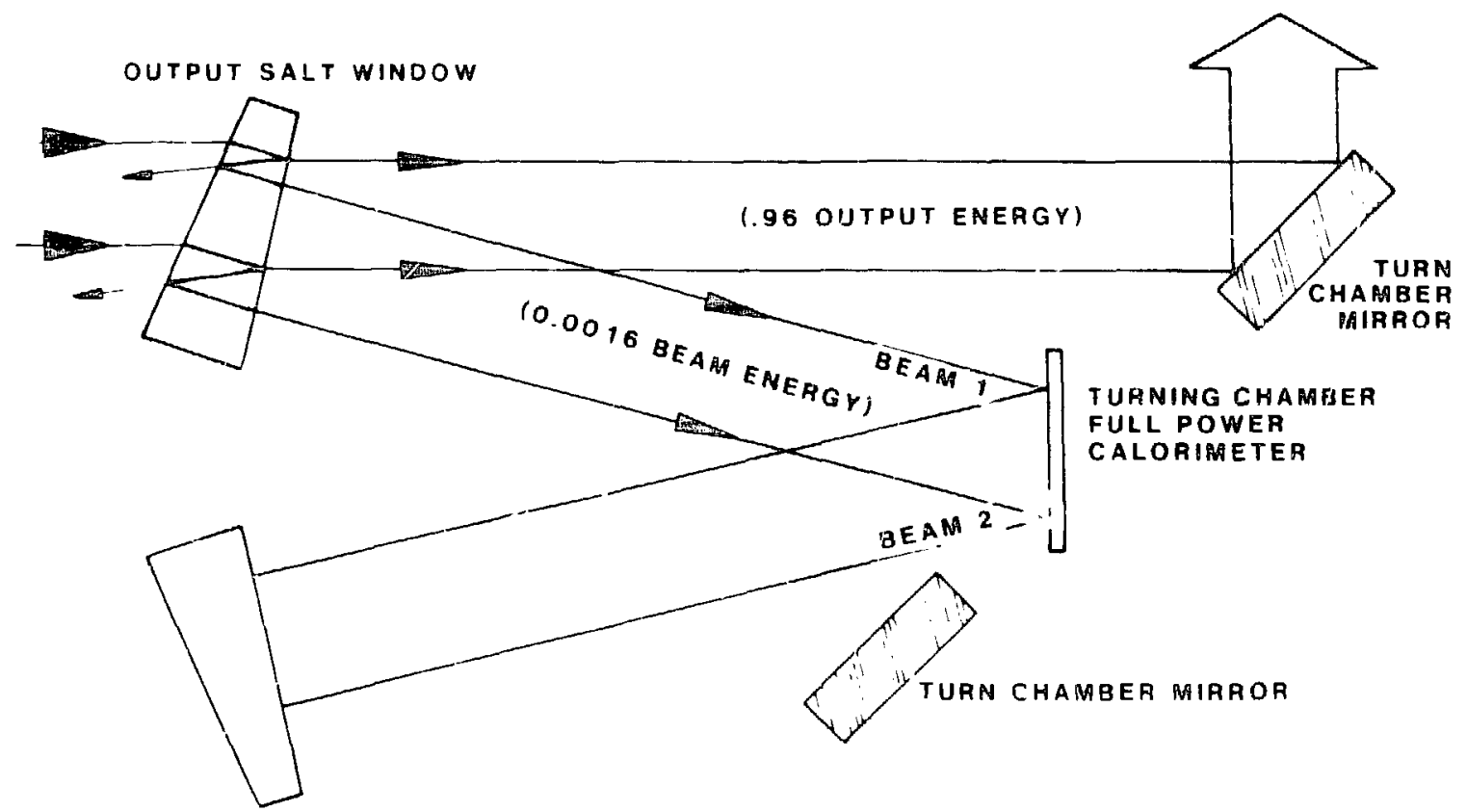

Fig. 17. The full output energy of the Antares power amplifiers is measured by sampling the Fresnel losses that occur at the output salt windows. About $4 \%$ of the incident light is reflected at each window surface. The output salt windows are fabricared and mounted so that the deubiy reflected beams from all 12 sectors overlap on a alorımeter located at the tum chamber. 
made to intersect at a point near the turn chambers. Since only about $4 \%$ of the incident beam energy is reflected at each surface, the energy in each sector beam was attenuated by a factor of about 0.0016 when it arrived at the turn chamber. This attenuation kept the total energy (12 sectors) arriving at each calorimeter below the damage threshold of the material used for the calorimeter.

A new type of calorimeter was developed to measure the wide aperture beams found in Antares. These calorimeters use a Kapton/copper laminate as the absorber material. Kapton is an excellent absorber at 10.6 $\mu \mathrm{m}$ and has a relatively high laser damage threshold. The iemperature risr of the copper backing is directiy related to the energy incident on the calorimeter to the degree that the calorimeters may be treated as a tertiary standard. Calorimeters of this design were inserted into each sector of the alignment system to characterize the output of the alignment system and to calibrate the operating calorimeters located at the turn chambers.

\section{SYSTEM CONTROL AND DIAGNOSTICS}

During the initial design of Antares, it was recognized that manual operation of the system would be extremely difficult because of the size and complexity of the system. We considered the experience of the preceding laser systems in the control requirements for Antares. The decision was made to tie all of the functions-charge and fire, alignment. Liagnostics, and so forth-into a common integrated contrni network. Because of the complexity of the system, the secision was also reached to have no hard-wired logic within the system but to base all of the system operation on software. The control system is based on a network (Fig. 18) of distributed computers. The in.egrated control computer, a PDP $i: / 60$, controls the timing and firing of $t^{\prime}$. system. Data archival is carried out by a PDF 11/70. Operation of each of the riain pulsed-power systems and of the optical alignment and beam diagnostics are carried ou i by three PDP $11 / 60$ minicompr:ters. The minicomputers download software and operating parameters to LSI-lI microcomputers located throughout the system. Communication between the LSI- 11 microcr:mputers is handled by the minicomputers.

The dat 3 are digitized and transmitted over fiber-optic cables throughout the computer network (a total of 40 $\mathrm{km}$ nf (ibers). ill operation data are archived and are on screens and available for future use. There are no traditional control panels. The interface between the oper- ator and the machine is established by means of touchsensitive display screens. The displays are created and can be changed through software programming.

\section{SYSTEM STATUS}

All of the subsystems in Antares were essentially complete in October 1983. At that time energy had been delivered from both alıplifiers into the target vacuum system at the $15 \mathrm{iJ}$-level. The quality of the beams produced by the powei amplifiers can be judged by tho burn pattern (Fig. 19) that was obtained at the turn chamber of beamline-2. When diffraction at the edges of the beams is taken into account, it can be seen that the output energy density is very uniform. The spatial coherence of the output ca: be judged by the Fresnel bright spot. occurring in the shadow of the mirror that injects the beant into the power amplifier (the notch in the outer edge of each individual burn pattern). By the end of December 1983, targets had been shot with all 24 beams. The present record energy delivered to target is $27.5 \mathrm{~kJ}$ in a 1 -ns pulse.

In the 12 months following the commencement of the target-shooting program, the operation of the Antares system has continued to improve. The system passed through a period of 6-8 months that can be classed as adolescence, during which problcms with the system were found and corrected and the confidence in the system was gained. The Antares laser has now become an adult. operational system. The preseni systern capabilitues are broad. The overall range of the capabilities of the system include the following:

- Delivery of $30-35 \mathrm{~kJ}$ to target in a I-ns pulse

- Variable pulse length from 1-10 ns

- Independent alignment of the individual beams to 24 positions within a $1-\mathrm{cm}^{3}$ volume

- Alignment precision to $40 \mu \mathrm{m}$

- Variable delay of pulse arrival at target $\pm 1.5 \mathrm{~ns}$ within one power amplifier \pm 100 ns between power amplifiers

The times to align the system are dependent on the alignment precision required by the experimenter. If the required tolerarice is greater than $200 \mu \mathrm{rn}$, then the system has been shot at 25 -minute intervals. When the best possible alignment has been required, then two 24 . beam shots and/or three 12-beam shots have been re corded in a single day. The key machine parameters are listed in Table 2, where a comparison is made between the design parameters and the achieved operating results. 


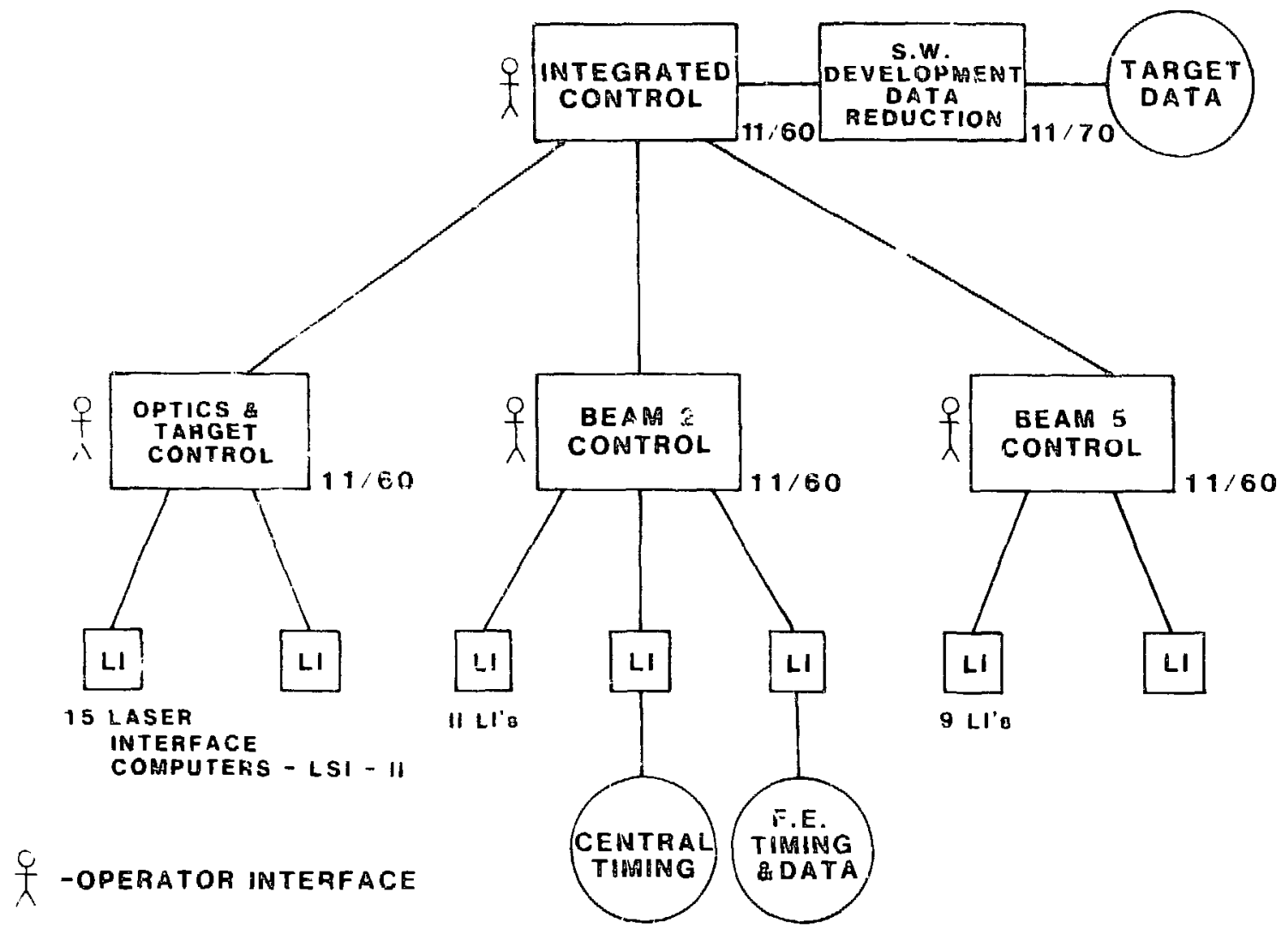

Fig. 18. In the Antares Integrated Control Network, over 40 computers are linked together to control the entire laser system. 


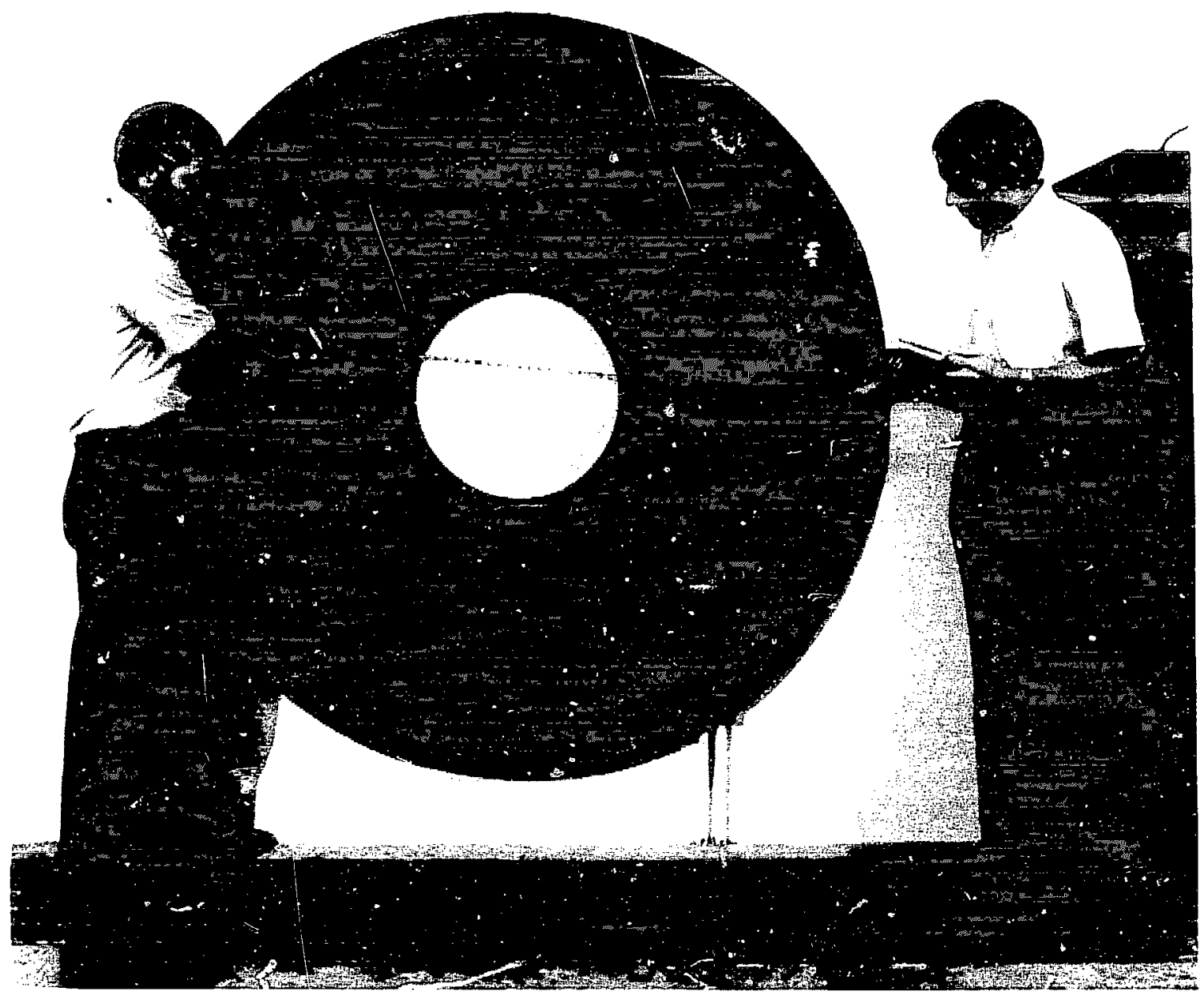

Fig. 19. The laser output from one power amplifier was recorded by burning this pattern into a sheet of photographic paper. The tota energy required to produce this burn pattern was about $8 \mathrm{~kJ}$ and was delivered in a single $1-n s$ pulse. The uniformity of the laser output can be judged from the uniformity of the burn pattern. 


\begin{tabular}{|c|c|c|}
\hline Parameter & Design & Operational \\
\hline e-Gun voltage & $525 \mathrm{kV}$ & $510 \mathrm{kV}$ \\
\hline e-Gun current & $25 \mathrm{kA}$ & $20 \mathrm{kA}$ \\
\hline e-Gun current density & $50 \mathrm{~min} / \mathrm{cm}^{2}$ & $40 \mathrm{~mA} / \mathrm{cm}^{2}$ \\
\hline Anode voltage & $550 \mathrm{kV}$ & $475 \mathrm{kV}$ \\
\hline Anode current & $220 \mathrm{kA}$ & $190 \mathrm{kA}$ \\
\hline Discharge current density & $7.0 \mathrm{~A} / \mathrm{cm}^{2}$ & $6.0 \mathrm{~A} / \mathrm{cm}^{2}$ \\
\hline Average electric field & $18.0 \mathrm{kV} / \mathrm{cm}$ & $15.5 \mathrm{kV} / \mathrm{cm}$ \\
\hline Pressure & 1800 torr & i 70 el torr \\
\hline$E / p$ & $10.0 \mathrm{~V} / \mathrm{cm} \cdot$ torr & $9.1 \mathrm{~V} / \mathrm{cm}$.torr \\
\hline Average gain length & 8 & 7.5 \\
\hline Average output fluence & $1.7 \mathrm{~J} / \mathrm{cm}^{2}$ & $1.3 \mathrm{~J} / \mathrm{cm}^{2}$ \\
\hline Energy & $20 \mathrm{~kJ}$ & $15 \mathrm{~kJ}$ \\
\hline
\end{tabular}

A vigorous target shooting prograrn is nc., under way. A fueled target such as the one shown in Fig. 20 has been shot in order to demonstrate the production of neutrons and to verify the operation of the various target diagnostic systems. Figure 21 is an open-silutter photograph of a shot where about $20 \mathrm{~kJ}$ of energy was directed onto a target.

During the testing of the $A_{2}$ ntares subsystems and their integration, most design goals have been attained. Not enough experience has been obtained to verify advertised or mean times between failures of the equipment. Some redesign was necessary, partictila ly to attain the voltage hold-off of the eiectron gun and to eliminate gain-fimiting parasitic oscillations in the power amplifier. Based on limited gain and energy measurements in the driver and power amplifiers, we now bclieve that Antares will attain its final goal. delivery of 35 - to 40 -TW pulses to inertial rusion targets. 


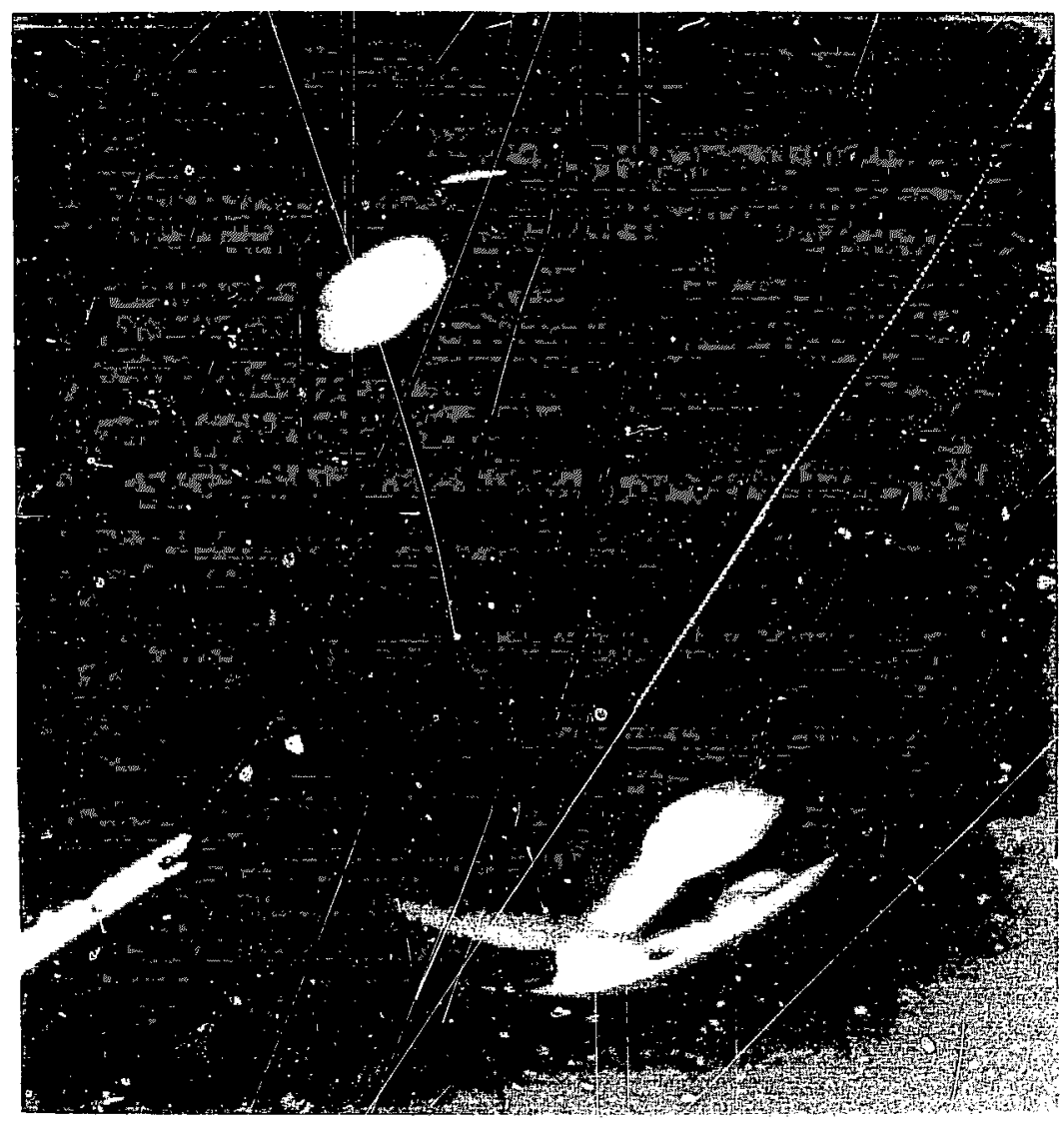

Fig. 20. A $200-\mu \mathrm{m}$-diam glass microballoon like the ones that would be filled with a mixture of deuteriuma and tritium for an energy extraction experiment. This particular microballoon (unfilled) was shot with only one beamlet and only about $1 \mathrm{~J}$ from the front end to demonstrate the size of a beamlet focused on the target. The hole drilled hy that shot can be seen on the right side of the microballoon. 


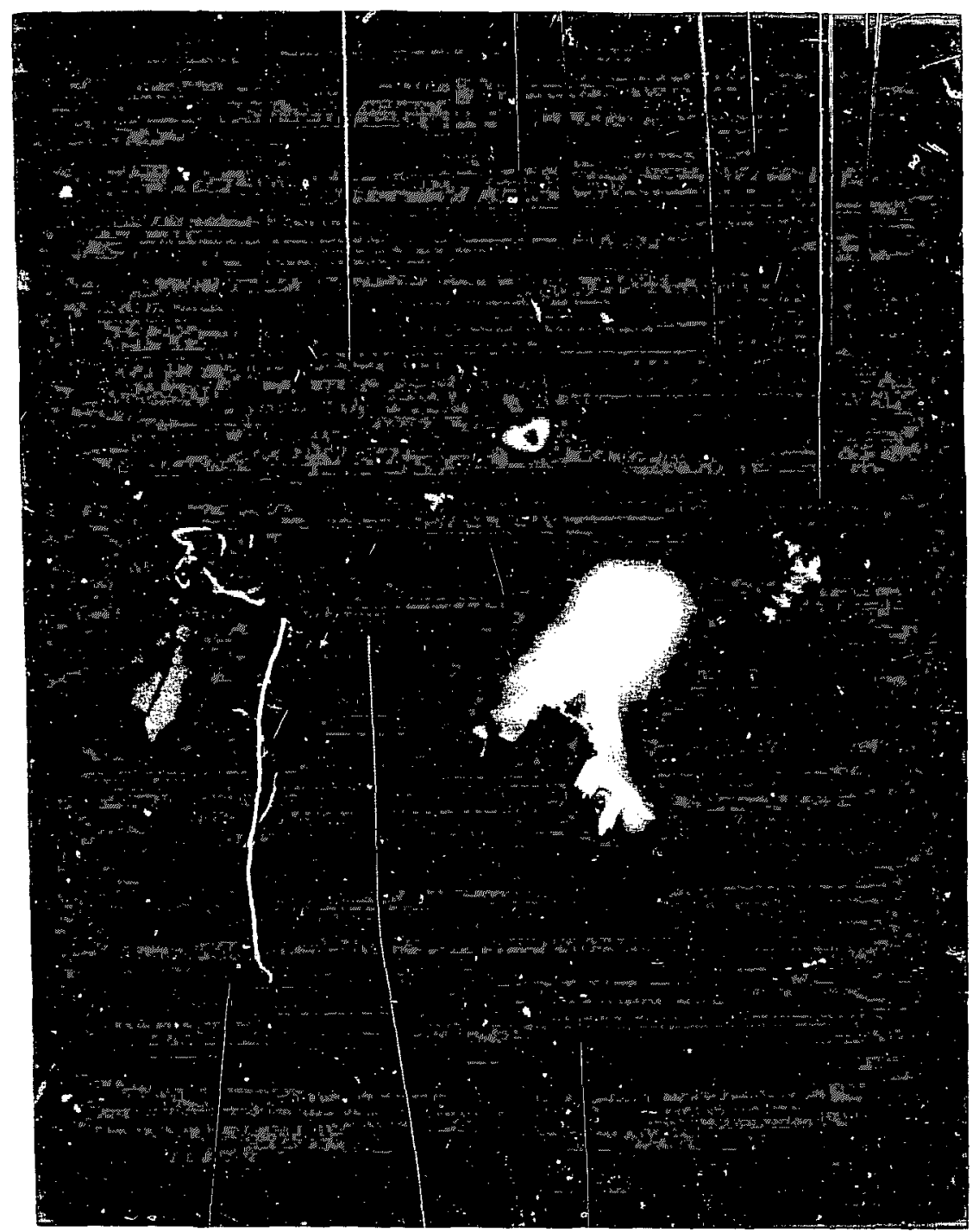

Fig. 21. Flash of light from a target shot with about $20 \mathrm{~kJ}$ of energy from Antares. The target insertion mechanism (on the lef:) , cnvered with a plasma sheath. The plasma sheath results from the interaction of the plasma and light froms ihe target with the surface of the target insertion mechanism. The object at the upper right is an $x$-ray pinhole camcra. The circular pattern on the lower right is the specular reflection off of the tool marks on the mounting plate located below the target. 


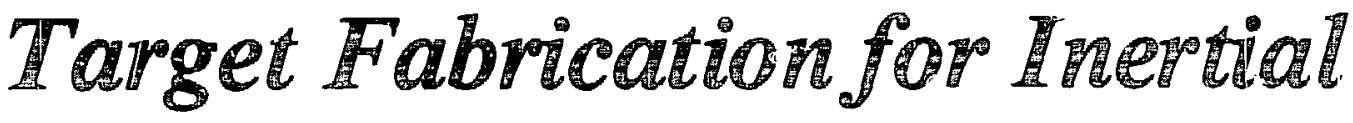 Confinement
}

\author{
by Richaidd Mah, David V. Duchane, and \\ Ainslie T. Young
}

\section{INTRODUCTION}

F usion targets, where: for the laser program or the particle beam jogram. are not difficult to picture iccause they are simply a series of concentric spherical shells. Yet realization of this simple design poses extraordinary difficuities. The targets are small, less than a millimeter in diameter overall. Each shell must possess specific properties ranging from strength at low densities to high atomic number and density for improved compression. The tolerances are exacting - 10 times more so than those common in the aerospace industry. No wonder we sometimes feel that the target designers are asking us to fabricate the impossible from some imaginary material! Every effort must be expended to meet the designers' demands, however, for their theories and computer codes can be verified only by experiments with real targets.

We have had considerable success in target fabrication, developing new technologies, new materials, and new tools. Wt will outline some of these developments and then briefly indicate the value of our efforts to other areas of technology.

\section{FUSION TARGETS}

Figure 1 shows the components of a typical fusion target. An overall view of much of our programmatic research and development can be gained by considering the fabrication of each component, beginning with the innermost.

\section{Tamper}

The sphericai she!l known as the tamper transmits implosive force to the deuterium-tritium fuel, theret,y compressing it to the density at which fusion occ. s. Lik all the other shells, the tamper must be extremel spherical, uniform in thickness, and smooth. (Thes requirements follow from the goals of symmetric im plosion and clean buris.) Impermeability to the gaseou fuel, strength, and resistance to embrittlement by the fue are primary considerations in selecting a tamper materia Commonly used materials include glass and metals rang ing from beryllium to gold. We focus here on meta tampers since glass tampers are available simply b selection from commercially available hollow glas spheres.

We fabricate metal tampers by electroforming. whic involves electrochemical deposition, electroplating, o autocatalysis of the desired metal on a sacrificic spherical substrate. Electroplating, which is applicable $t$ conducting substrates of metal or metallized glass, is well-developed technique made unusual in this applic tion by the exacting tolerances on thickness uniformit by the requirements on both physical and mechanic properties, and by the necessarily small size of th sutstrates. Figure 2 shows an apparatus we have d veloped for electroplating spherical substrates small than $590 \mu \mathrm{m}$ in diameter. Constant, random motion c the spneres between two cathodes assures that all sul faces are uniformly plated.

Conducting substrates larger than $500 \mu \mathrm{m}$ in diamete are electroplated in a track-plating apparatus. Unifor plating is achieved in this apparatus by the rando I olling of the spherical substrates, each within a restricte area, over the surface of a flat cathode (the track). A advantage of track plating is that the plated material mechanically worked during plating (by contact of th spheres with the track) and thus has superior mechanic strength and fine grain structure. 


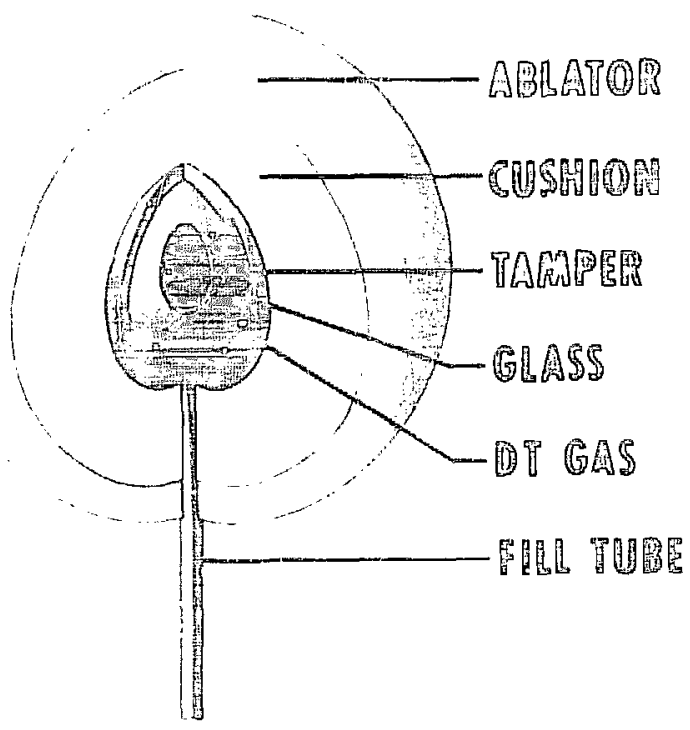

Fig. 1. Schematc of a typical fusion target. Each component plays a specific role in conversion of energy from the driver to th implosive energy necessary for fusion of the fuel.

Tampers can be deposited on nunconducting suhstrates by rutocatalysis. or "electroless" plating. In this process the substrate catalyzes reduction of the metal irnm a metastable solution. Although finding a substrate matcrial with appropriate catalytic action is difficult. the process nroduces tampers of very uniform thickness and with very smonth surfaces (Fig. 3). Electroless plating of substrates smalle: than $500 \mu \mathrm{m}$ in diameter is performed in an apparatus in which the spheres are kept in constant. random motion in a reaction chamber by alternating the tou direction of the metastable solution. ..?

Some recent target designs call for tampers of metals. such is beryllium? and aluminum, that cannot be deposited from aqueous solution. We are, therefore, in. vestyating electrodeposition from organic solvents and possibly molien salts. The major obstacle in this research is the hazardous nature of such deposition media. in the meinume, wher piscesses called physical vapor depo. wion (PVD) and chemical vapor deposition (CVD) are ured wo provide tampers from a number of materials not currentl stanah!e by electrochemical processes.

The term PVD includes elestron-beam evaporation. putermy and ion plating. Tu achieve uniform depoitmon on spherical targets with these line-of-sight processes, we have desagned double-axis rotators that irien: all areas of the sphere equally (on the average) toward the vap or source. ${ }^{3}$ We are also investigating the possibility of le vitating smalier targets during coating by use of a vibrating "bouncer pan" ur with a molecular beam (Fig. 4, ${ }^{4}$ Nevertheluss, the PVD process is harnpered by its low deposition rate $(<0.001 \mathrm{in.} / \mathrm{n})$ and the limitation of being able to process only 1.12 targets at a time.

In the CVD process, a gaseous compound of the rnaterial for the target ( $\mathrm{WF}_{6}$, for example) undergoes chemical reduction or thermal decomposition on the surface of the target substrate. The desired metals are depesited on small targets in a bed of substrates fluidized by the reactive gas. This produces a nuidized bed, a churning mass of particles that is formed by directing a flow of gas upward through a volume of particles of appropriate size and density. The continuous, random motion of the fluidized substrates ensures that all surfaces are exposed (on average) to the same temperature and gas flux, and very uniform coatings are deposited even on substrates with complex geometry. In addition, the collisions among the substrates provide a peening action that helps to break up growth patterns in the deposit that would normally produce large, columnar grains. As a result, the coating usually consists of fine. equiaxed grains and is thus strong ant smooth. ${ }^{5}$ 


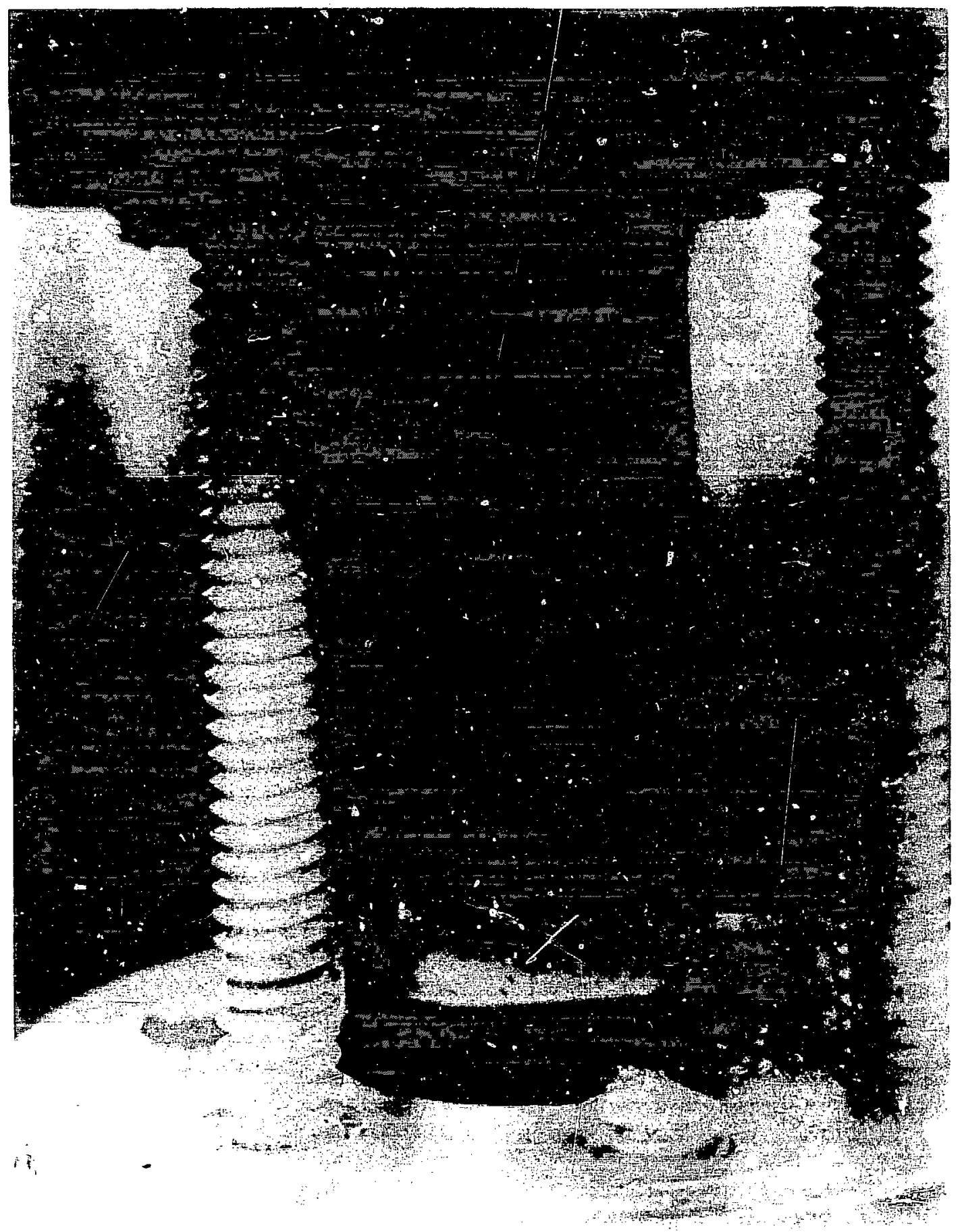

Fig. 2 An apparatus for electroplating small (less than $~ 500 \mu \mathrm{m}$ in diameter) conducting spheres. The spheres are forced against one and then the other of two cathodes of fine-mesh screen by alternating the flow direction of the electroplating solution. The constant, random mction of the spheres assures uniform deposition of the plated material. The large polyethylene balls prevent agglomeration of the small spheres. 


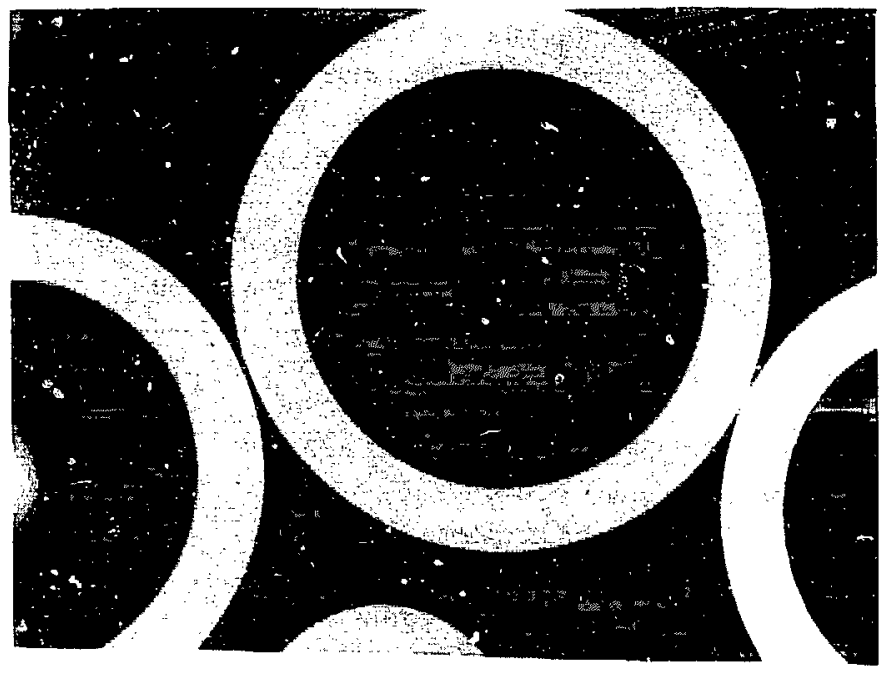

Photomicrographs of 14 - $\mu$ m-thick nickel tampers ed by autocatalysis (electroless plating) on a al glass substrate. The intact sphere (a) and the od sphere (b) show, respectively, the great smoothad the thickness uniformity that can be obtained is electrochemical process.

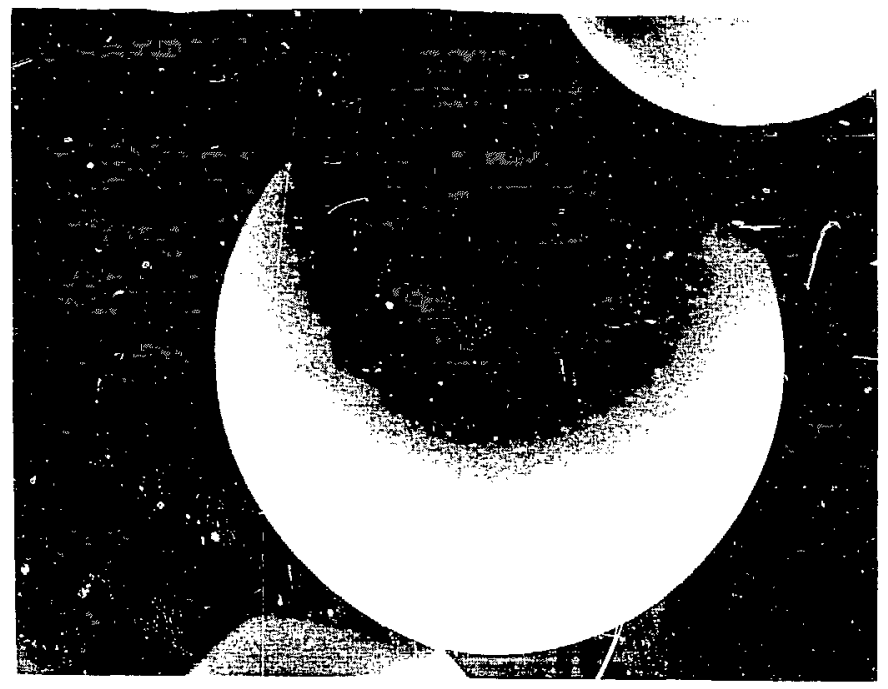

Fig. 4. Magnifying video display for monitoring physical vapor deposition on spherical substrates levitated by flow of gas llirough an arrey of fine capillary lubes. Shown on the stieen iaiong with its shadow) is a levitated hollow glass sphere. which is $300 \mu \mathrm{m}$ in diameter.

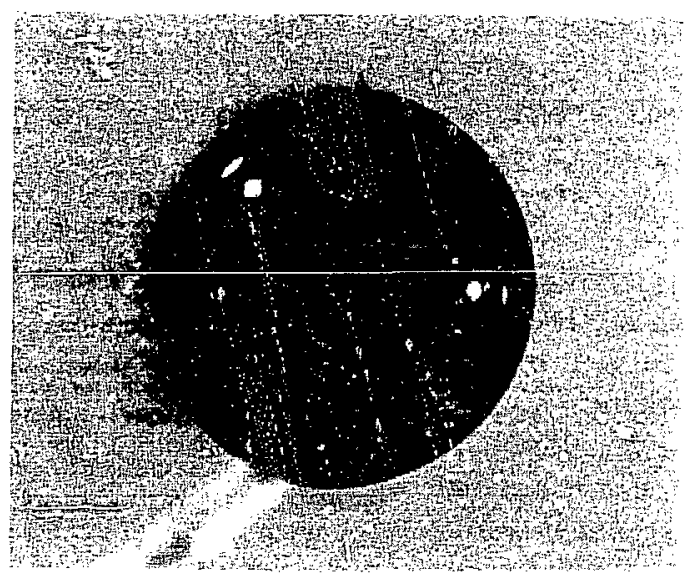




\section{Cushion}

The cushion provides a stand-off distance between the tamper and the ablator. This cushion, ideally a "structural yoid," originally consisted of full-density low-Z polymeric materials, but more recently has been made of rigid plastic foam. When the cushion layer consisted of a full-density polymer, two techniques were used. The first technique involved micro- and ultraprecision machining to produce hemishells that were later assembled around the tampers. This technique will be described in more detail in connection with fabrication of ablators. The second technique (described here) involved two methods for direct deposition of a polymer from the gas phase: the low-pressure plasma (LPP) process and the vapor-phase pyrolysis (VPP) process.

In the LPP process, a radiofrequency voltage between two electrodes produces a low-pressure plasma of the monomeric material, which then polymerizes on the tamper surface. Small tampers (less than $500 \mu \mathrm{m}$ in diameter) are levitated above vibrating shaped electrodes during the coating process. Pulsing in the monomeric vapor and maintainance of the electrode temperature below $\sim 10^{\circ} \mathrm{C}$ forms very smooth layers with thicknesses between 1 and $\sim 60 \mu \mathrm{m}$ from such monomers as $\mathrm{p}$ xylylene and cycloacetetraene (Fig. 5). ${ }^{6}$

In the VPP process, p-xylylene is pyrolytically degraded to the reactive diradical species, which then polymerizes over the surface of the object to be coated. We have adapted this well-known process to deposit uniformly thick. smooth layers on stalk-mounted or levitated tampers. The uniformity in thickness of a 600 $\mu \mathrm{m}$-thick cushion layer prepared by the VPP process varies by less than $3 \%$, and its smoothness is marred only by a few micrometer-sized "bumps." Greater surface smoothness of thinner layers can easily be obtained by increasing the turbulence of the reactive gas. We have, for example, prepared defect-free coatings up to $70 \mu \mathrm{m}$ thick with surface irregularities of less than $0.1 \mu \mathrm{m}$ from peak to valley. ${ }^{6}$

Both the sarface smoothness and thickness uniformity of these depccited cushion layers can be greatly enhanced by lappinis techniques originaily developed for metals and since refined for polymers. These techniques will be discussed below in connection with the fabrication of ablators.

More recently, better hydrodynamic performance has been achieved by using plastic foam. These must have very low density ( $0.05 \mathrm{~g} / \mathrm{cm}^{3}$ or less) and small cell size (less than $5.0 \mu \mathrm{m}$ in diameter). They must be fres of impurities and amenable to fabrication to precise tolerances. Such foams were unknown before their development at Los Alamos.

We make foams of this type by cooling a homogeneous polymer solution in such a manner as to induce an inverse phase separation (that is, of solvent from solution) at the temperature at which the polymer and solvent become immiscible (the spinodal point). The polymer forms a foam matrix, and the solvent forms discrete, uniform droplets in this matrix. Removal of the solvent droplets from the matrix generally results in a porous, open-cell plastic foam in which the voids are replicas of the solvent droplets. The foam is rigid when made with polymers that are inherently inflexible. The density of the foam is determined by the concentration of the polymer solution.

We have prepared small-cell, low-density, rigid foams from such polymers as polystyrene, poly(4-methyl-1penetene), tradenamed TPX, and substituted celluloses and starches. Hemishells of these foams can be made by inducing the inverse phase separation in an appropriate mold. In some cases, micromachining can be used to improve the dimensional properties of the foam material. Foams made from TPX have a very uniform structure. but the low compression strength of such foams (about $10 \mathrm{lb} /$ in. $^{2}$ ) makes them difficult to machine to exact tolerances by normal techniques. To eiiminate this difficulty, we have developed a polymer-solvent system in which the solvent droplets are solid at room temperature. The solid solvent reinforces the delicate cell walls and thus protects them from damage during machining. The solvent is then extracted with alcohol.?

\section{Ablator}

The energy of the radiation from the driver is deposited in the shell known as the ablator (see Fig. 1). The absorbed energy causes material to be ejected outward from the ablator, producing a "rocket" effect that transfers inward momentum to the tamper, which results in compression of the fuel. Ablators are fatricated from various materials primarily by machining.

The small sizes and close ciimensional tolerances typical of fusion targets dictate that ablators be machined with ultraprecision tools. With such tools and singlecrystal diamond tool bits we can. for example, produce surfaces with a peak-to-valley roughriess of $0.05 \mu \mathrm{m}$.

An important aspect of precision lurning is the constancy of rotational speed and accurate rutational moion 


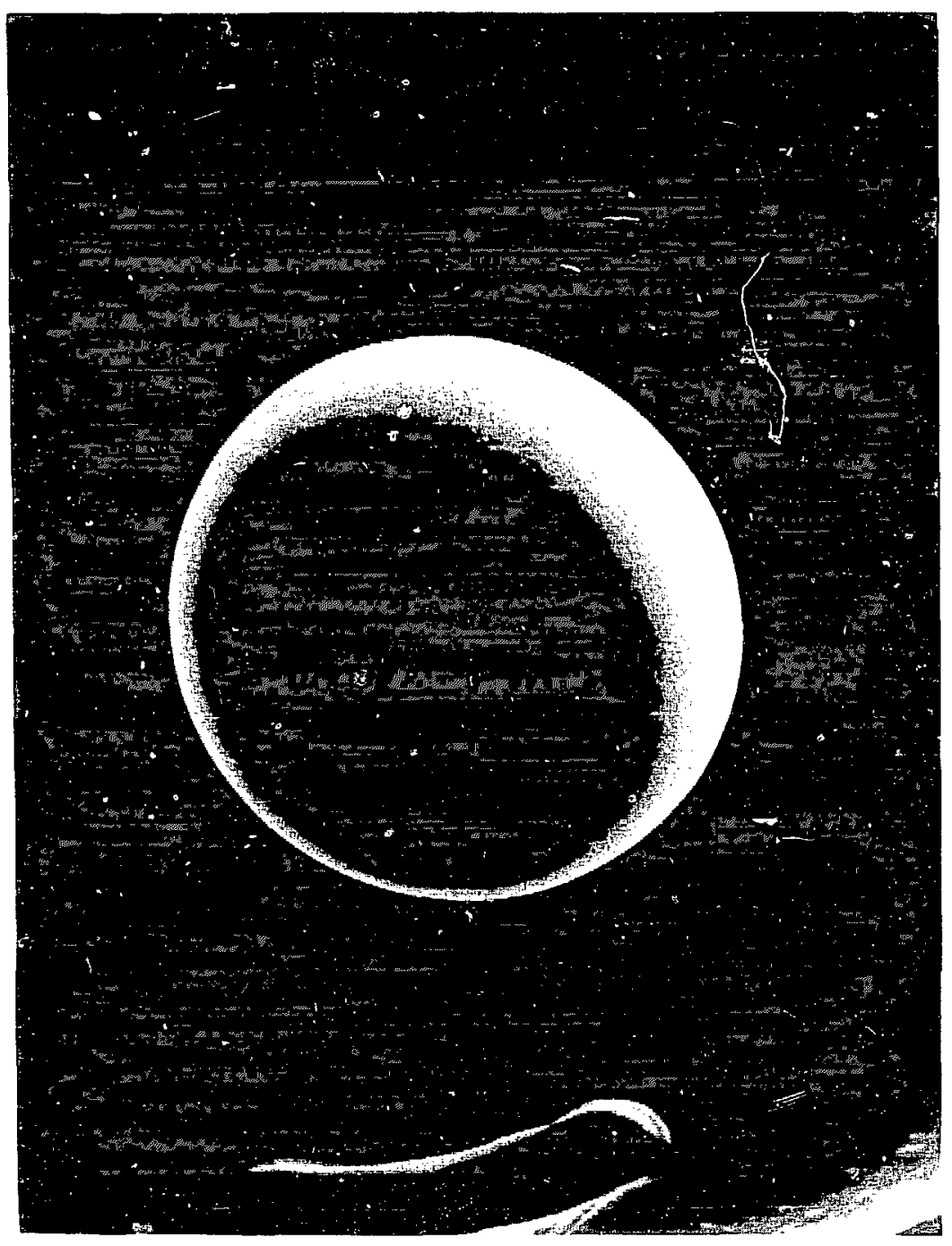

Fig. 5. Scanning electron micrograph of a 300- $\mu \mathrm{m}$-diam hollow glass sphere laser fusion target coated by the low pressire plasma process with a 5 - $\mu \mathrm{m}$.thick layer of parylene. Note the surface smooihness of the coating.

provided by air-bearing spindles. W: have available two small lathes with air-bearing snindles and vibrationisolating magnetically coupled drive motors. We also have a larger ultraprecision lathe (Fig. 6i, built to Lus Alamus specifications by Pneumo Precision, inc., that combines the leatures of air bearing spindles and slideways with those of computer control. This lathe is used for metal optics and other high-precision fabrication at Los Alamos. To increase our machining capability we are now preparing specifications for another lathe with a programmable rotary axis and the same accuracy as that of the P'neumo lathe.
Although the surface finishes produced by ultraprecision turning are excellent, they can be further improved (except on many face-centered-cubic materials) by lapping, or polishing with fine abrasive particles. By using submicrometer-sized grit and air balancing to check the progress of the lapping, we can obtain surfaces that are smoothed to about $0.003 \mu \mathrm{m}$ peak to valley. I hese are the smoothest surfaces attainable with a mechanical technique. Shapes other than spheres or hemispheres can be lapped with special equipment. ${ }^{8}$ 


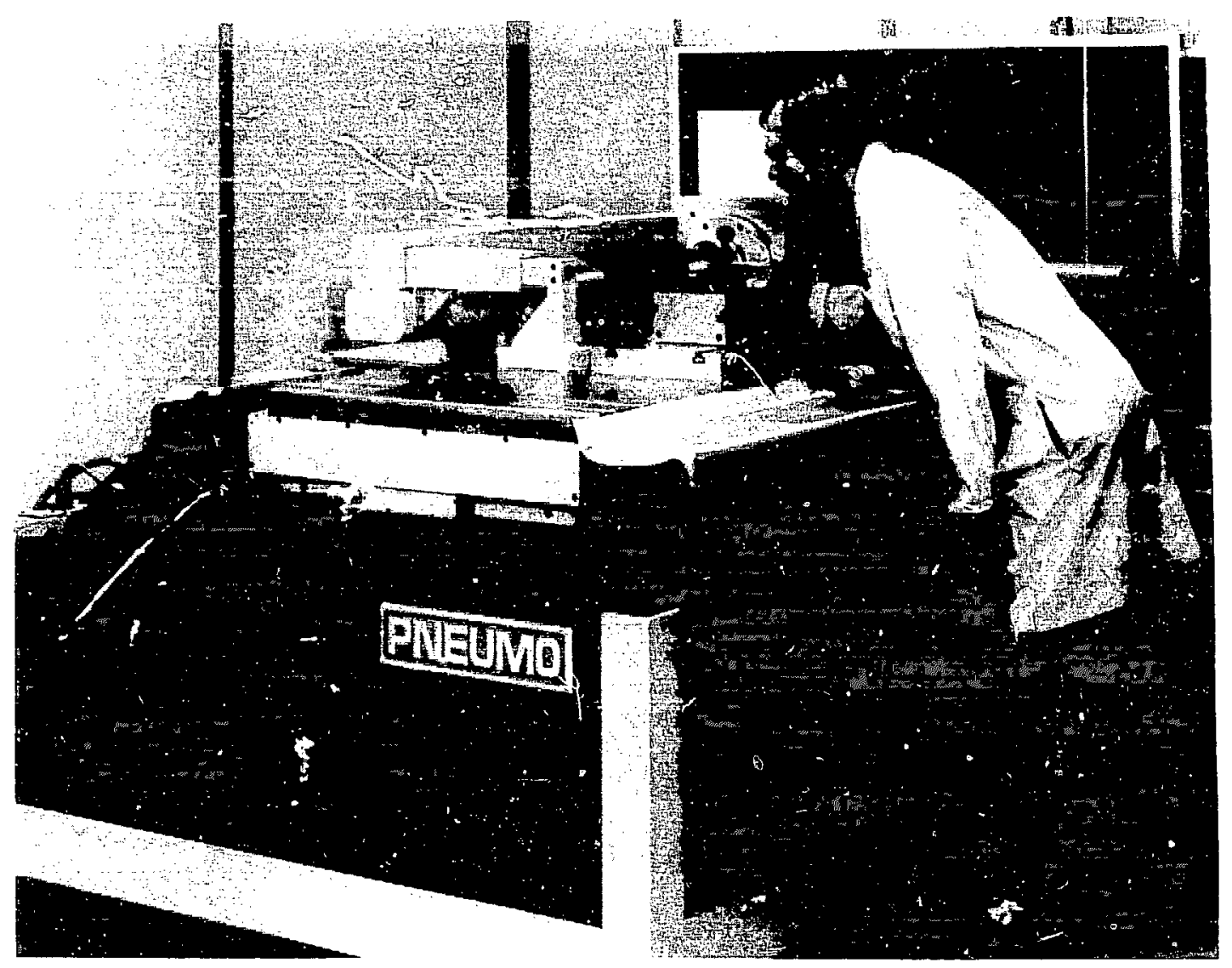

Fig. 6. Uluraprecision lathe built to Los Alamos specifications by Pneumo Precision, Inc. The position feedback systeln of this machine which is based on laser interferometry, has a resolution of $0.025 \mathrm{pm}$. Its low-îriction air-hearing slideways allow $p$ recise contouring, and any surface of revolution can be generated by sing' programming. The slideways are mounted on a mascive granite block. which in tum is supported on three air-bag vibration isolators. With this lathe we use single-crystal diamond tool bits for machining most plastics and some nonferrous metals. We use cubic boron nitride and conventional carbide tool bits for machining materials (such as beryllium) that are incompatible with diamond tool bits.

\section{OTHER A.PPLICATIONS}

It is not surprising that our efforts on the multifaceted task of target fabrication have led to materials and technologies of interest and benefit to other research and development programs. We mention briefly a few of these other applications to indicate the variety of problems in which materials science plays a fundamental role.

- The polymeric foams developed for the cushion layer may benefit biomedical research. Porosity is considered essential in synthetic veins and arteries to provide anchoring points for biological species that inhibit the natural tendency of the body to build up a thick layer of scar tissue around foreign objects. The synthetic vascular prostheses currently in use, primarily woven Dacron and Teflon structures, have pores that vary widely in size. In contrast. the cells of the foam produced by the inverse phase separation process are remarkably uniform and can be made in the range of $25 \mu \mathrm{m}$ in diameter, the sizc believed to be most desirable for biological uses. ${ }^{9}$

- A process called chemical infusion, which was developed for smoothing polymerir surfaces, may also benefit biomedical research. Experiments on laboratory animals have indicated that silicone rubber veins infused by this process with stearate ions may be rejected less often than are untreated irtificial veins. ${ }^{10}$

- Miniature coaxial cables fabricated in support of inertial confinement fusion experiments may simplify surgical removal of brain tumors. High-frequency electrical signals transmitted through such a 
cable, which is less than $0.1 \mathrm{~mm}$ in diameter, may enable the destruction of cancerous cells without damage to surrounding tissue. ${ }^{11.12}$

- Laser welding, the method used to braze fill tubes onto tampers, may greatly simplify fabrication of radioactive components. Components of plutonium, for example, must be welded in a glove bnx, and with conventionas techniques this condition necessitates contamination of expensive welding equipment. In contrast, a laser welding beam could be directed onto the component through special glove-box windows and could even be transported throughout a facility along optical fibers.

- Tuols like those developed for uniformly costing target components by physical vapor deposition are now routinely used for coating rare-earth radiochemical tracers on experimen:al nuclear weapon components.

- High-strength single-crystal whiskers, which were fabricated and studied for possible use as support structures in laser fusion targets, are now the key component in the Los Alamos structural ceramics program. These whiskers of silicon carbide or silicon nitride have tensile strengths approaching 4000000 psi. Composites of such whiskers and ceramic materials have very high tensile strengths and high resistance to fracture.

Clearly, target fabrication and the associated research and development encompass many diverse endeavors. Formerly, these were pursued at several scattered Laboritory sitcs. But since the fall of 198; most of our activities have been gathered in a single, specially designed building, the Target Fabrication Facility. This lacility includes clean rooms for target assembly, a laboratory in which the targets are filled with the deuterium-tritium fuel, a machine shop, and laboratories for electrcplating and physical and chemical vapor deposition, for developing and producing polymer foams. for laser welding and solid-state bonding, and for characterizing the various rarget components. Beyond the obvious advantages of space and equipment, the facility allows grtater ease of communication among those involved in materials science, fatrication techriology, and materials characterization.

\section{REFERENCES}

1. A. Mayer and W. Doty, "Advances in Plating Discrete Hollow Glass iMicroshells," Technica!
Digest Conference on Inertial Fusion, Optical Soc. of America. San Diego. California, 1980. p. 96.

2. A. Mayer and D. S. Catlett, "Plating Discrete Microparticles for Laser Fusion Targets," Plating and Surface Firishing, March 1978, p. 42-46.

3. G. A. Keeves, "Physical Vapor Deposition onto Small Spheres," Technical Digest Conference on Inertial Fusion, Optical Soc. of America, San Diego. California, 1980, p. 60.

4. S. F. Mayer, "Metallic Coating of Microsptieres," $J$. Vac. Sci. Tech. 18 (3). 1198-1204 (1981).

5. D. W. Carroll and W. J. McCreary, "Fabrication of Thir-Wall, Freestanding Inertial Confinement Fusion Targets by Che.nical Vapor Deposition," $J$. Vac. Scl. Tech. 20 (4), 1087-1090 (1992).

6. R. Liepins, M. Campbell, J. S. Clements, J. Hammond, and R. J. Fries, "Plastic Coating of Microsphere Substrates." J. Vac. Sci Tech. 18 (3). 1218-1226 (1981).

7. A. T. Young, D. K. Moreno, and R. G. Marsters. "Preparation of Multishell ICF Target Plastic Foam Cushion Materials by Thermally Induced Phase Inversion Process." J. Vac. Sci. Tech. 20 (4). 1094- 1097 (1982).

8. R. L. Rhorer. "Update on Precision Machining at Los Alamos," Proc. Soc. Photo-Optical Instzum. Eng., San Diegn. California. Vol. 433. 1983, p. $107-111$

9. J. C. Stanley. W. E. Burkel, S. M. Lindenauer, R. H. Bartlett. and J. G. Turcor=, Eds., Biolugic and Synthetic i'ascular Prostheses (Grune and Stratton. New York, 1982).

10. D. V. Duchane, "Polymeric MandrelsSupersmooth Surface by an Infusion Process." $J$. vac. Sci. Tech. 18 (3) -1183-1186(198!).

11. W. L. Bongianni. "Fabrication and Performarce of Strip-Centered Microminiature Coaxial Cable," IEEE Proceedings, Vol. 22 (Sepiamber 1984).

12. M. Kachmer. Fd. "Brain Tumors Succumb to New Microwaye Probes." Microwave and RF. November 198.3, p. $30-46$. 


\title{
Heavy-Ion Fusion
}

\author{
by Roger $O$. Bangerter
}

I $t$ is widely beileved that many proposed fusion devices would produce energy if they could be made large enough. Stars and thermonuclear weapons are two demonstrated examples ti:at support this belief. In pas iuular, because thermonuclear explosives are inertially confined. the scientific feasibility of inertial confinement fusion (ICF) has already been te ronstrated at large scale. Sufficiently large magnetic fusic.. Jevices will almost cer:ainly also produce net energy.

Large devices, however, might not make good environmental. engineering, or economic sense. Thus, for commercial appications of fusion, the important question is not whether fusion can produce energy - but rather,

San a fusion energy systom be built that makes good environmental, engineering, and economic sense?

Fusion scientists have always implicitly recognized that this yuestion 13 the important one, but in recent years the question has been stated explicitly with increasing trequency and urgen.y.

For example. Lawrence Lidsky of MIT has been extremely critical of some proposed fusion devices because, in his opinion, they do not make good engineering sense. His article, ${ }^{1}$ widely quoted in the media, has further focused attention on the importance of engineering and economics in fusion energy production.

\section{ION BEAMS}

Interest is increasing in what we call heavy-ion fusion because it is an approach that appears to offer significant advantages for a real fusion power-a plant that would make good erivironmental, engineering, and economic sense.

The idea of igniting inertial-fusion targets with intense ion beams is not new. Researchers realized in about 1975 that more than 50 yaars of engineering development had culminated in long-lived, efficient accelerators that could reliably produce ion pulses at high repetition rates (1 heriz and higher). Furthermore, the beams from these machines are now focused over large distances anto small targets. An example of such a machine is the LAMPF accelerator at Los Alamos (Fig. 1). Experimants now reenforce the theory that interaction between the ion beam and the target is devoid of many of the problems (such as iow absorption and hot clectrons) that have plagued laser fusion.

From an engineering standpoint, such accelerators appeared suitable to drive an inertial fusion power plant. Accelerators were not considered seriously earlier be cause ali existing accelerators were low-power devices by inertial fusion standards. However, by 1976 many re searchers concluded that the power problem was not fundarnental. ${ }^{2}$ In fact, it appeared that several types of acceltrators, including radio-frequency linear ac. celerators (rf linacs.. $5 \%$ i: inrotrons, induction linacs. and pulsed-power dicder might provide adequate power (greater tha: $)^{14}$ in short bursts of about 10

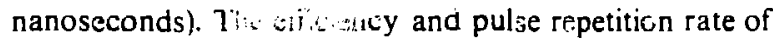
synchrotrons are 10u'cumpared with the linac systems. Therefore, research on synchrotrons for ICF has nearly stopped, but research programs are active in the other three technologies in the United Sitates and abroad.

Although pulsed-power diodes are included among the accelerators that might provide adequate power, we must recognize that diodes lack the demcistrated lifetime and pulse repetition rates of if linacs and induction linacs. There are other important distinctions between ion diodes and the other accelerators. Diodes are limited in voltage. At present, ion diodes operate at less than 10 megavolts (MV). The highest voltages contemplated are several times that. If we assume $10 \mathrm{MV}$ is typical, we must accelerate 10 megamperes (MA) of eur.ent to achieve the required $10^{14}$ watts. By contrast, both if linacs and induction linacs are multistage devices. There is no difficulty. in principle, in accelerating ions to arbitráriiy high ene gies. There are, however, two practical difficulties: : ost ard icn range. Cost is discussed later; ion range, which is discussed now, is an important considera lion shat bears directly on the type of accelerator that can be used for inertial fusion. 


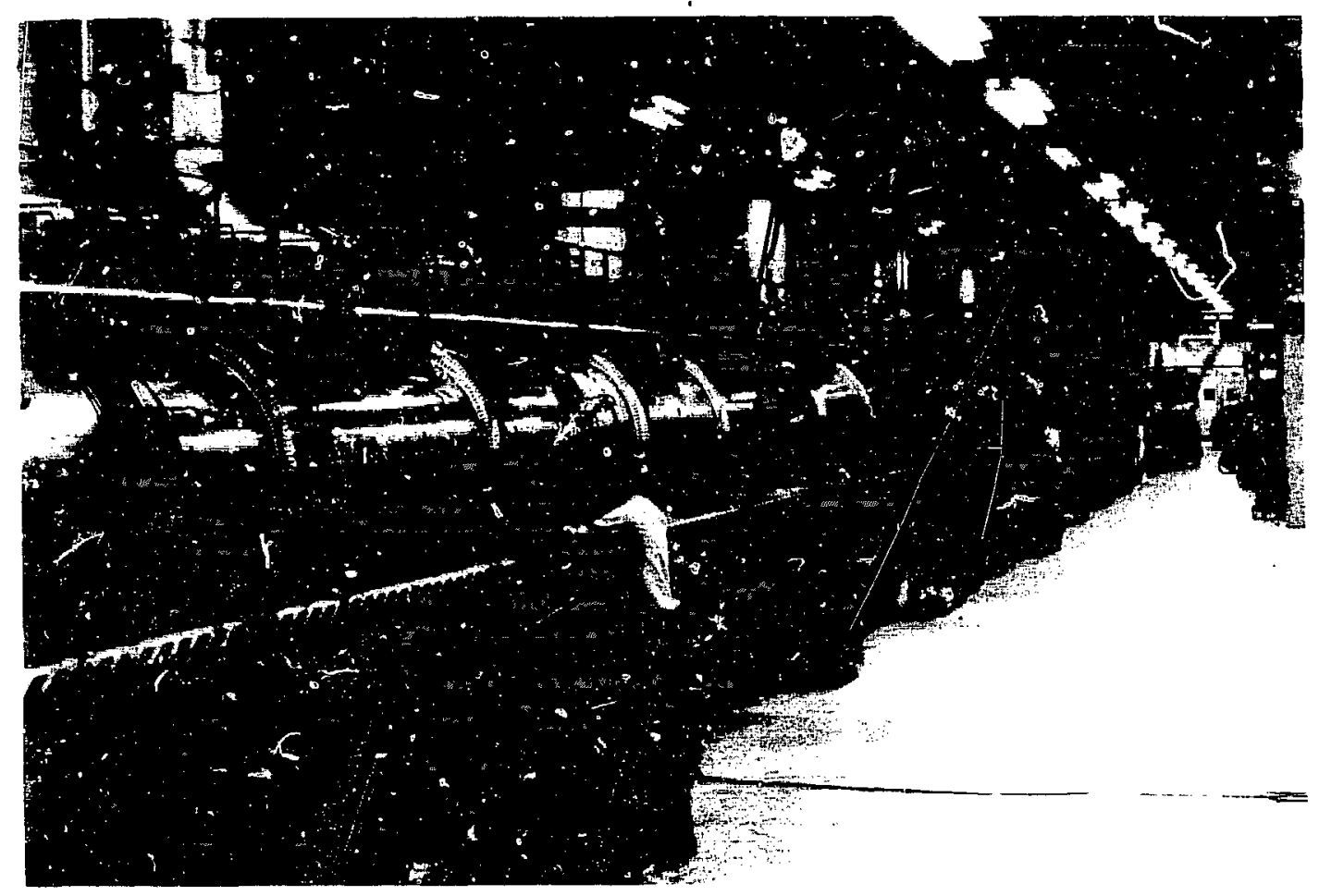

Fig. 1. LAMPF, the Clinton P. Anderson Meson Physics Fncility at Los Alamos, a one-half-mile-long linear accelerator that produces a beam of protons with a maximum energy of 300 -million electron voits and an average beam current of I milliampere.

\section{Range}

To provide adequate pressure to implode a target. specific energy deposition in the outer layers of the target must exceed about 20 megajoules per gram ( $\mathrm{MJ} / \mathrm{g})$. For a spherical target, the specific energy ceposition is given by $E / 4 \pi r^{2}$, where $E$ is beam energy (in $M J$ ), $r$ is the target radius (i $r_{2} \mathrm{~cm}$ ), and $R$ is the ion range (in $\mathrm{g} / \mathrm{cm}^{2}$ ). For typical values of $E$ (several $M J$ ) and $r$ (several $\mathrm{mm}$ ), $R$ cannot exceed several times $0.1 \mathrm{~g} / \mathrm{cm}^{2}$ and still satisfy the $20 \mathrm{MJ} / \mathrm{g}$ criterion. In fact, ion ranges less than $0.1 \mathrm{~g} / \mathrm{cm}^{2}$ are preferable as such ranges increase the specific energy deposition for a given target radius. High specific energy is expected to improve target performance.

Sun range as a function of ion kinetic ent -gy is shown for several different elements in Fig. 2. If we take 0.1 $\mathrm{g}^{\prime} \mathrm{cmi}^{2}$ as the desirable ion range, protons or other light ions are acceptable : vith kinetic energies in electror, volts (eV) up to atout 0.01 gigaelect on volts $(\mathrm{GeV})$; however, ieacy ions. such as lead ions, allov kinetic energits up to about $10 \mathrm{GeV}$. Diodes, with $10 \mathrm{MV}$ that can accelerate protons to kinetic energies of $0.01 \mathrm{GeV}$, are thus suitable

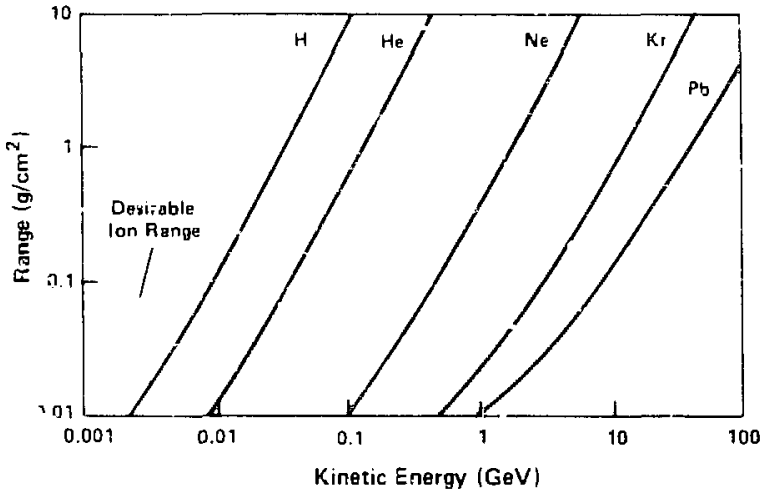

Fig. 2 Ion range as a function of kinetic energy for a variety of ions. Heavy tons allow the use of multistage accelerators with high energy beams but, at the sume time, keep the range of the ions in the desired region, at or below $0.1 \mathrm{~g} / \mathrm{cm}^{2}$. 
for light ions; multistage accelerators, for heavy ions. (In common usage "light-ion fusion" is nearly synonymous with "ion-diode fusion," and "heavy-ion fusiọn" is nearly synonymous with "multistage-acce'erator fusion.") At 10 $\mathrm{GeV}$ the current required to achieve $10^{14}$ watts of singly charged heavy ions is only $10 \mathrm{kA}$ rather than the $10 \mathrm{MA}$ required for diodes. This difference of three orders of magnitude in current requirement is one of the distinctions between light-ion fusion and heavy-ion fusion.

Perhaps a more fundamental difficulty with diode devices is that all of the beam energy is delivered at a single accolerating gap. This results in highly stressed conponents, and it is not surprising that these diodes have not demonstrated adequate lifetime or pulse repetition rates. Some suggested diode-fusion devices employ multiple accelerators to obtain the required beam power. This relieves, out does not eliminate, the problem of highly stressed components. In the remainder of this discussion, we consider oniy multistage accelerators for which, fortunately, $10 \mathrm{GeV}$ is a reasonable $:$ itage.

There is also a major difference in the requirements for the two types of multistage accelerators; with if linacs, storage rings (sometimes called accumulators) are necessary to achieve the required $10 \mathrm{kA}$, whereas induction linacs should not require storage rings. In fact, the design current for the advanced test accelerator (ATA) electron induction linac now coming or-line at the Lawrence Livermore National Laboratory is $10 \mathrm{kA}$. and about $7.5 \mathrm{kA}$ has already been achieved.
The U.S. program focuses on induction accelerator research, whereas the Europeans and Japanese are studying $f$ linacs for heavy-ion fusion. Although much of what we discuss here applies to both types $r$ nuitistage accelerators, we will emphasize the induction linac, which, in its simplest form, can be thought of as a series of transformers with the beam as the secondary. The current to the primaries is provided by capacitors and switening devices (Fig. 3). Each transformer is energized only while the beam passes through it.

We now return to the question of cost.

\section{CONSIDERATIONS FOR POWER PRODUCTICN}

To understand why accelerators are attractive for fusion, it is necessary to examine the requirements that must be satisfied by any commercial power plant. We consider three general issues relevant to nearly all power plant technologies: environmental acceptability, total plant capacity (usually roughly proportional to plant cost C), and the cost of electricity $r_{e}$.

Although ail methods of energy production have some environmental impact, fusion should be acceptable Fusion plants can, in principle, be several orders of magnitude better than fission ieactors in limiting undesirable production of long-lived radioactive isotopes. '

As mentioned er.:lier, the size of a iusion device is important. Power companies prefer plants of small cost

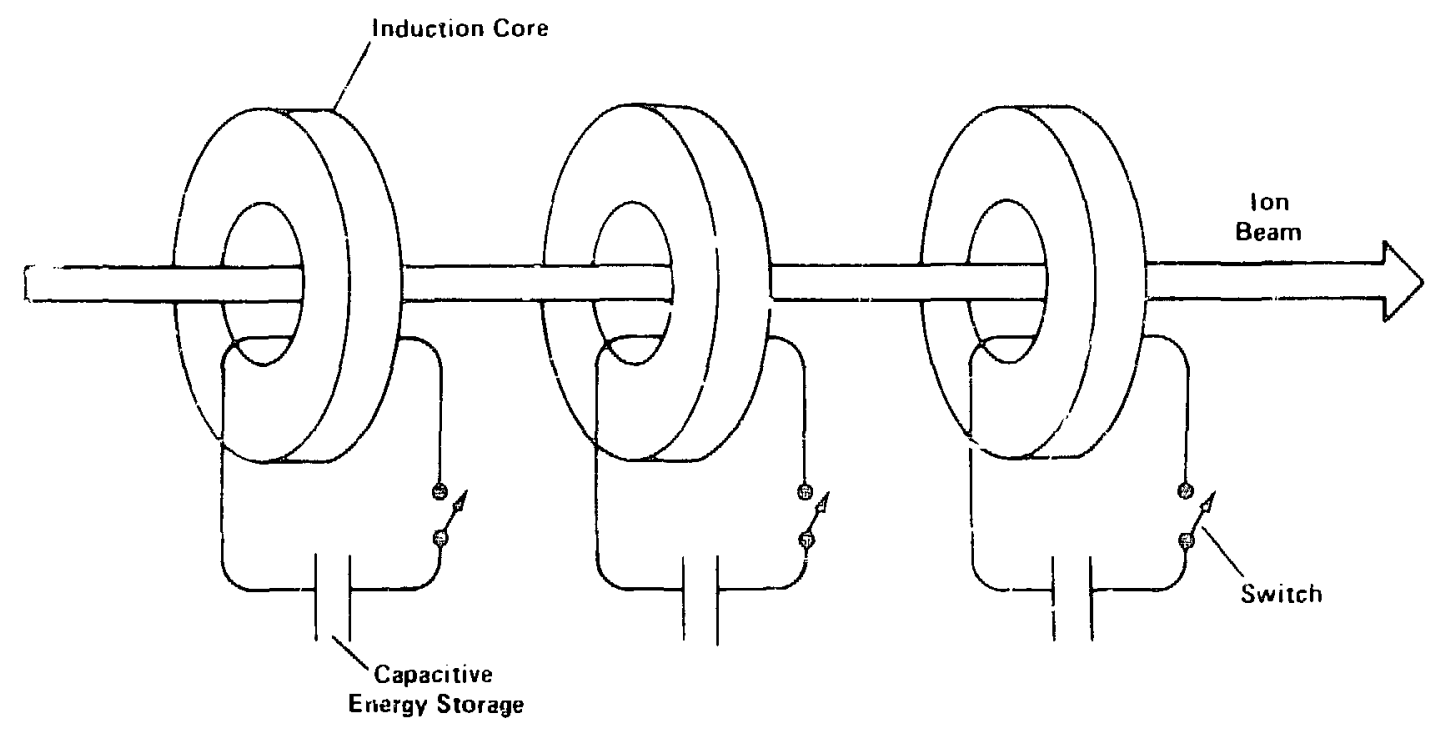

Fig. 3. The induction linac as a series of transformers with the beam acting as the secondary. 
and capacity. There are several ieasons for this preference. Large power plants, costing perhaps several billion dollars, can severely strain the capital assets of even large utility industries. Moreover, large plants, which offer little flexibility in terms of siting and market size, are slow to construct (a major problem for the nuclear industry) and produce large perturbations on the power grid when they go off-line. Perhaps more importantly, high cost can be a severe impediment to the introduction of any new energy technology.

The third concideration, cost of electricity, can be, illustrated conveniently with total plant cost in twodimensional space. as shown in Fig. 4. Consider a reference point located at $C$ equal tn $2.5 \times 10^{9}$ dollars and $r_{e}$ equal to 50 mills per kilowatt hour. If either is plant or electricity can be made more economically, it is beneficial: therefore, the areas labeled "bettcr" and "worse" are clearly better or worse, respectively, than the reference point. The situation is not as clear in the areas labeled with question marks. For exainple, a utility might choose to incur higher plant capital costs to reduce the price of electricity.

We now examine the features of a power plant that would enable us to move in the "better" direction, and one ICF feature is particularly attractive. The driver and reaction chamber are relauvely independent components. and the fusion energy release occurs in a volume of space smallir than the volume of a pea. The energy yield of each target can be varied by large factors; the plant capacity can be varied by even larger factors by adjusting both the yield and the pulse repetition rate. Thus. in principle, there is much flexibility in the design of ICF piants. and smail plants are possible. For this feature of ICF to to usetul. tin dryer and, eactor chamber must be ine:pensive $x_{1}$, at the cost of electricity can be low.

The size and cost of a reaction chamber are related directy to target yield so that smaller yield means smaller

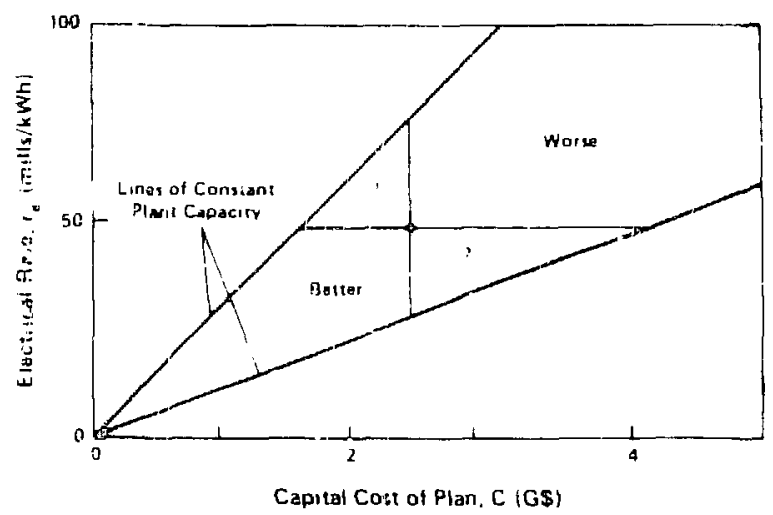

cost. Also, both target yield and driver cosı decrease with drive. energy. Thus it should be economically advantageous to reduce driver energy and, at the same time. increase the driver repetition rate to obtain the desired thermonuclear power output. There are, however. limits to this strategy. One is the maximum repetition rate at which the driver can operate. Another irsolves the product of driver efficiency $\eta$, target energy gain $G$, and the efficiency $\epsilon$ of the reactor and turbines. A simpufied power flow diagram of an ICF power plant is shown with these parameters in Fig. 5. Clearly $\eta \mathrm{G} \epsilon$ must exceed unity to produce any net power. Because $\epsilon$ is typically about one-third. $\eta G$ is required to be greater than three.

To have enough net power to be economical, it is commonly assumed that $\eta G$ must exceed about 10 . In reality, the lower limit for $\eta \mathrm{G}$ depends on the cost of drivers, reaction chambers, turbines, generators. and so forth. The simple criterion of $\eta \mathrm{G} \leq 10$ is adequate here.

Target gain calculated as a function of driver energy ${ }^{3}$ is shown in Fig. 6. These curves are based on single-shell targets, and the range shown is appropriate for both heavy-ion accelerators and short-wavelength lasers. More complex multiple-shell targets give somewhat higher calculated gain, but the physics uncertainties are larger and fabrication is apt to be more difficult. Figure 6 shows that attempts to lower driver cost by decreasing driver energy also decreases target gain $G$; the requirement for ${ } G$ can then oe met only if the drive: has high efficiency $\eta$.

Quantitatively, we expect that $\eta$ should be about 0.25 for heavy-ion accelerators. We could then satisfy our $\eta \mathrm{G}$ criterion with target gains as low as 40 . By contrast. a $\mathrm{KrF}$ laser is unlikely to have an $\eta$ that exceeds 0.05 ." which would require a target gain in excess of 200 . Using the top curve in that figure, we see that a gain of $40 \mathrm{can}$ be achieved with an input energy of $2 \mathrm{MJ}$ so that the yield would be about $80 \mathrm{MJ}$. On the other hand. to achieve a

\footnotetext{
- Since this document was prepared. Los Alamos advances in KrF technology indicate that efificiencies of over $10 \%$ are possible.
}

Fig. 4. Cost consideratıons for $\stackrel{m}{-n}$ electric gererating plant. Because the cost of electricity is approximatel; proportional to electnc output divided by capital cosh sloping lines (two of whuch are shown) correspond to constant electric output. The point represenis a typical fusion power plant design. Heavy-ion fusion is attractive because it has many attributes that might allow power planis to operate in the region labeled "bet:er." 


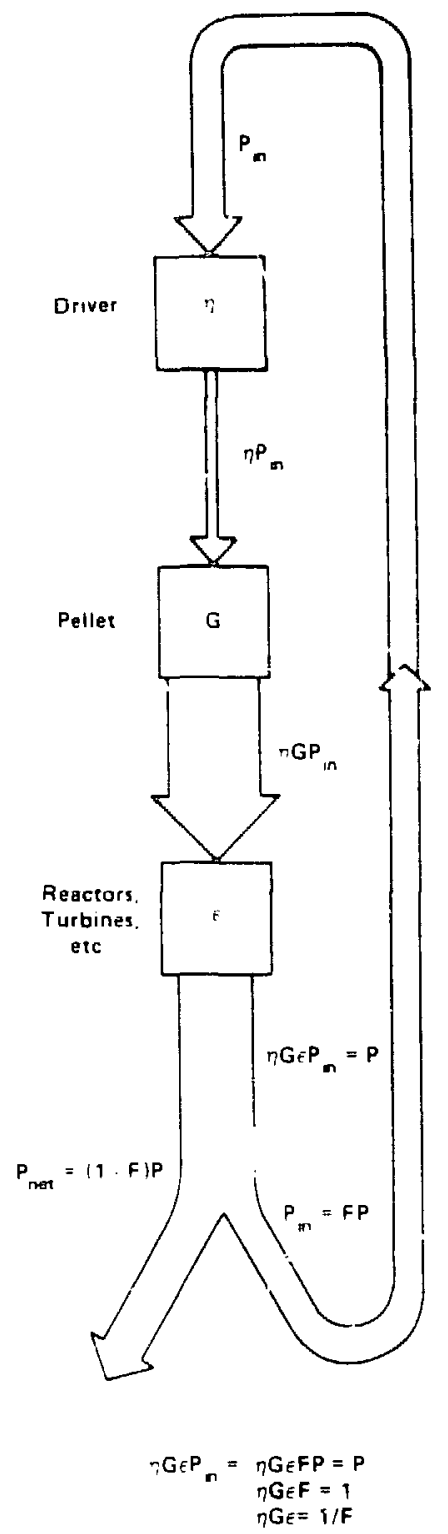

Fig. 5. A simplificu power flow diagram for an ICF plant. Of the priwer $P$ coming from the iurbines. a fraction $F$ is recirculated $\left(P_{(n)}\right)$, and a fraction ( $\mid-F)$ becomes the useful power generated by the plant $\left(P_{\text {ner }}\right)$. The oquations show the relationship between $F$ and the product of dnver efficiency $\eta$. target energy gал $G$. and the efficiency $\epsilon$ of the reactor and turbines.

gain of 200 requires more than $20 \mathrm{MJ}$ of input energy, and the correspondin!; yield would be greater than 4000 MJ. Thus, the size and cost of a reaction chamber is expected to depend strongly on driver efficiency. Because $\mathrm{KrF}$ lasers are one of the most attractive laser candidates

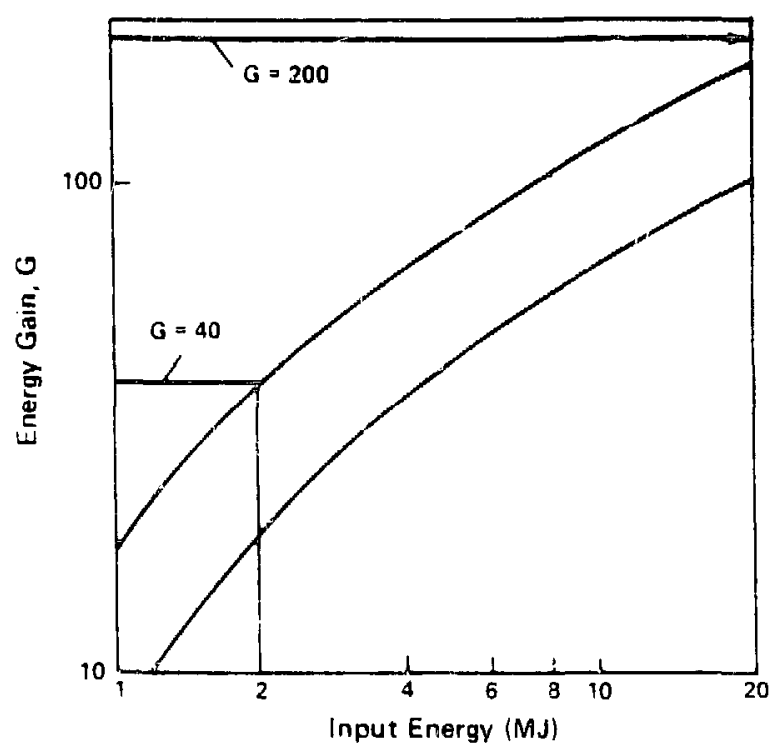

Fig. 6. Calculated target gan as a function of drivie energy for sungle shell targets. The range shown is aporopriate for both hesvy-ior accelerators and short-wavelength lasers. However, the high efficienc) of the heavy-ion accelerators should allow these devices to operate a a gain of around 40 rather than at the gain of 200 expectec for lasers thereby lowering the size and cost of the reaction chamber.

for ICF. it is evident that high efficiency is a very important advantage of accelerators over lasers. Design ing single-shell targets with gains higher than those showr in Fig. 6 might be possible. Indeed, some current esti mates of target gain exceed those shown: however, ever the gains illustrated in the figure have not been demon strated. Accelerator fusion has a larger safety factor thar does laser fusion. based on these considerations.

\section{Driver Requirenzents}

Other driver features that enable one to move in thi "better" direction are reliability and durability. Lov reliability increases the cost of electricity because $i$ reduces the output of a given capital investment. Goor durability is important because high repair and replace ment costs increase the cost of electricity. The drive features necessary for economically explr,ting thi favorable flexibility of ICF are

1. low cost.

2. high repetition rate.

3. high efficiency.

4. reliability, and

5. durability. 
These five features lead us to the consideration of accelerators as inertial fusion urivers. Features 2 through 3 have either been demonstrated at existing accelerators or appear to be reascrable extrapolations of existing technology.

The cost of accelerators, the first item on the list, has ben, and remains, an issue. It might be possible to build a 3-MJ accelerater for about five hundred million dollars. Although this compares favorably with proposed laser system , pursuing cost reductions is still very important

There are additional requirements that must be satisfied. Some of these, such as favorable implosion dynamics, are nearly driver-independent and are discussed elsewhere in this review. However, three ilriverdependent requirements are discussed here:

1. high peak power,

2. an ability to focus the beam, and

3. acceptable physics for the beam-target inter action.

Peak beam power greater than $10^{14}$ watts is required, as noted earlier, and this has not been demonstrated at existing accelerators. The second requirement, the ability to focus the beam, is related to high power. Target gain decreases with increasing focal-spot radius-at least for focal radii greater than 1 or $2 \mathrm{~mm}$. Adequate focusing has been demonstrated at existing accelerators but never at the power levels required for ICF. These two requirements are discussed in the next section.

The interaction 'jetween beam and target must behave properly, in that the beam nust be absorbed but must not penetrate too deeply into the target. Moreover, the beam must not produce secondary particles or photons that preheat the fuel and prevent efficient compression. The beam-target interaction has been a criucal issue for laser fusion, and some laser-light wavelengths are almost certainly unacceptable for ICF applications. Discussion of the interaction physics follows the next section.

\section{HIGH POWER AND BEAM FOCUSING}

High power and beam focusing are related because, although sinple ions can be aimed with very high precision, the mutual interaction of a large number of charged particles (space-charge forces, for example) complicates matters. This mutual interaction is also a problem for acceleration. Luring acceleration the beam must be held together both transve; sely and lorigitudinally by applied focusing fields. Transvers? focusing is usually provided by electrostatic or magnetic lenses. Long- itudinal focusing is provided by time-varying accelerating iields. If beam power or current is increased, the repalsive space-charge forces of the beam ultimately overpow's the applied focusing fields. In some cases the interaction of the beam with the accelerator structure can produce instabilities that limit the beam power to values far lower than the limit set by the lens strength.

\section{Phase-Space IDensity}

Liouvile's theorem also sets important limits on power and focusing. As it is usually applied to accelerators. this theorem states that the six-dimensional phase space density of ions at the end of the accelerator cannot exceed the phase-space ciensity at the ion source. (Beam-cooling mechanisms that violate this condition do not seem applicable to heavy-ion fusion.)

The target radius limits the two transwerse spatial dimensions of the focused bear. For a given energy, the peak power requirement limits the lungitudinal extent of the beam. Because of effects such as third-order aberrations, $v$ it can be realized with focusing lenses puts an upper limit on the angular divergence of the beam approaching the target Because angular divergence is roughly proportional to transverse beam momentum. this translates to corresponding upper limits on transverse beam momentun. "Chromatic" aberrations of the lens sysiem (the fact that ions of different momenta have different focal lengths) piace an upper limit on the spread of longitudinal momenta in the beam. Thus. the target plus the final lens system place an upper limut on the sixdimensional phase-space volume of the beam emerging from the accelerator. This correspunds to a lover lumit on the phase-space densiry of the beam because the number of tons is fixed by target considerations. Fortunately, the phast-space density available from practical ion sources is orders of magnitude larger than the lower limit so that the Liouvilie constraint can, in principle, be satisfied. However, in all real accelerators there is some growth in occupied phase volume.

For miany applications it is useful to consider sixdimensional phase space to be a product of three two dimensional phase spaces. The volume or area of each subspace is referred to as emittance: the growth of cccupied phase volume. as emittance growth.

One important question for heavy-ion fusion is whether the phase volume constraint can stili be satisfied in the presence of realistic emittance growth. There are several potential sources of emittance growth. For if 
linacs, a large number of turns are used in the storage rings to obtain the required current amplificacion. In this process of multiturn injection, some unoccupied phase space is unavoidats, mixed with occupied phase space resulting in substantial emittance growth-probably a factor of 2 or more in each transverse dimension. This source of emittance growth does not exist for induction linacs.

Other potertial sources of emittance growth in both if and induction linacs include non.inear space-charee forces, imperfections in the accelerator, and instabilities that involve interactions between the beam and the acceleraing structure or interactions between the beam and any residual g,as in the reaction chamber from the previous explosion. Many o ${ }^{*}$ these effects have been stuc anaiycicatly and numerically with encouraging resuits, but perhaps the most encouraging comes from a series of experiments in progress at Lawrence Berkele: Laboratory. In these experiments it has been possible to transport a highly space-charge-dominated beam through a transport channel containing more than eighty elecrrostatic lenses wichout observable emittance growth.

\section{Experimental Results}

As all real beams have nonzero emittance. the particles in a beam will, in general. not move exactly along the axis of a transport channel. If a single ion is injected off-axis (in position or angle), it oscillates about the axis with a frequency (or wavelength) determined by its charge, mass, and momentum and by the strength of the lenses (restoring force). We call such oscillations betatron oscillations. If a large number of parcicles are injected into a channel, their mutual repulsion works against the restoring force of the lenses. and the wavelength and amplitude of the betatron oscillations increase relative to the singleparticle values.

It is convenient to compare the relative importance of the lens-focusing forces and the space-charge forces by comparing the parameters that appear in the equation for the betatron ireciuency $\omega$ :

$\omega^{2}=\omega_{0}^{2}-1 / 2 \omega_{1}$

Here $\omega_{0}$ is the betatron frequercy in the atsence of space charge, and $\omega_{b p}$ is the so-called beam plasma frequency given by

$\omega_{b p}=\sqrt{ } 4 \pi n q^{2} / m$. where $q$ and $m$ are, respectively, the ion charge and $m$. and $x$ is the ion density of the beam. As ion density space charge increases, the plasma frequency increas reducing the betatron frequency. It would not be surpl ing if beam instabilities occur when $\omega_{b p}$ becomes co parable to $\omega_{0}$. Indeed, analytic theories, based on ide ized particle distribution functions, show that this is case.

Another way of characterizing the strength of focus is in terms of "phase advance." Most commonly, lenses in an accelerator are quadrupole. These len have the property of focusing in one transverse direct and defocusing in the orthogonal transverse direstion. alternating the orientation of the lenses so that in e: plane every other lens is focusing or defocusing. a called alternating gradient channel is obtained. It is v known in optics that when a focusing lens is preceded followed by a defocusing lens of equal strength the effect is focusing. Thus an alternating gradient chan fucuses in both transverse dimensions. Each pair quadrupole lenses (including the contiguous lens spaci is referred to as a cell. The change in phase of betatron osrillations as the beam passes through one : is called the phase advance per cell $r$, which is denotec in the absence of space charge.

In general, small values for $\sigma / \sigma_{0}$ are desirable beca they correspond to high current. Unfortunately, anal! theory predicts the onset of unstable regions as this $r \varepsilon$ is lowered.

We now compare the results of analytic theory $u$ the experiments performed at the Lawrence Berke Laboratory. Figure 7 is a plot of $\omega_{b p}{ }^{2}$ as a function $\sigma / \sigma_{0}$ for various values of $\sigma_{0}$. The parameter $\omega_{b p}{ }^{2}$ is $u$ : because, for a fixed ion charge, mass, and velocity, $t$ quantity is proportional to the ion density $n$ and thu: proportional to the current density of the beam. Note $t$ $\sigma / \sigma_{0}$ decreases from one to zero, that is, the ph: advance per cell changes from the case of no spi charge $\left(\sigma=\sigma_{0}\right.$ ) toward the case of higher beam curre (lower $\sigma / \sigma_{0}$ ratios). (The experiments were perform with cesium ions at about $160 \mathrm{keV}$. The cell length $v$ $30.48 \mathrm{~cm}$ so that $\sigma_{0}=90^{\circ}$ corresponds to $\omega_{0}^{2} \approx 3 \times 1$ $/ \mathrm{sec}^{2}$.)

In Fig. 7 the regions of if : bility predicted by analy theory are the dotted parts ut the curves. Experimen results are the rectangular areas, with stable regic shown in black and unstable regions shown in whi Remarkably, the beams are more stable than predict allow:ng lower values of $\sigma / \sigma_{0}$ than we thought possil 


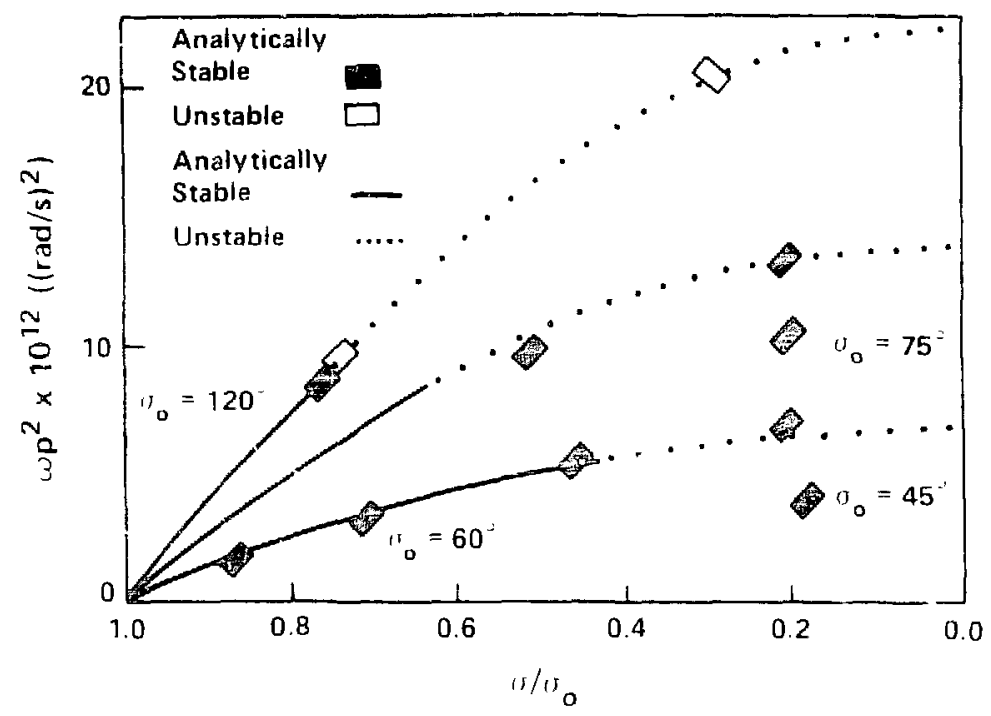

Fig. 7. Beam stability. When we compare the results of analyuc theory (curves) and the Lawrence Berkeley Laboratory experiments (boxes). we see that the beams are more itable than predicted (note the expermentally stable regions on the two lower curves). The parameter $\omega_{b}$ is the plasma frequenty, and $\omega_{b p}{ }^{2}$ is proportional to the beam current density. The parameter $o$ measures the change !n phase advance per cell of the betatron ascillations; $\sigma_{o}$ is the phase advance in the absence of any space charge ifigure adapted from Ref. 4).

several years ago. Fortunately, the experiments are consistent with receni numerical simulations that use nore realiste dist.ibutior. functions than does the analytic theory.

The new results are encouraging because they imply that for a given emittance. the beam current can be increased by a factor of a few over the value assumed severa! years agc. In particular. for a given total beam energy. higher current allows a reduction in the volume of magnetic material required for the induction cores. thereby lowering the cost. Alternatively, the new results allow a decrease in phase-space volume for a given amount of magnevi: material.

Even with these new. higher limits on transportable power, other techniques must be developed to ensure the generation of high-quality, well-focused heams carrying the $10^{i 4}$ watts required for $\mathrm{ICF}$. One of these te inniques uses miltiple beams; a second. "pressure windows."

\section{Multiple Beams}

For the required $10^{14}$ watts. it seems besi. at present. to accelerate each beam in its nwn focusing tiens system but to use common induction cores. Thus each core shown in Fig. 2 would have multiple beams going through it. The experiments at Lawrence Berkeley were performed with a single beam. Moreover, there was no attempt at longitudinal focusing or acceleration. Los Alamos and Law. rence Berkeley are now constructing an experiment, designated the multiple-beam experiment. to test these features. About three years will be required to complete the experiment.

\section{Pressure Windows}

Calculations show that space-charge forces are not strong enough to prevent beam focusing; however, instabilities may occur when the beam propagates through residual material in the reaction chamber. As the beam passes through the gas. a plasmia is produced that might generate some of the well-kn * beam-plasma instabilities. Most conceptual studies of heavy-ion-iusion reactors avoid this issuc by designing reactors with low enough residual pressure (less than $10^{-3}$ to $10^{-4}$ torr) that insufficient plasma is formed to generate instahilities. 9.6 This conservative approach may not be necessary. It has been suggested that "pressure windows" may exist where 
focusing is possible in the presence of gas.' For example. between about $10^{-3}$ and $10^{-1}$ torr, we expect the so-called two-stream instability to be a serious problem. However. between about $10^{-1}$ and 1 torr, the two-stream instability should be collisionally suppressed, and propagation may be possible. Between about 1 and 10 torr, enough plasma is produced to charge neutralize the beam. In this case, the magnetic fields of the beam may cause it to self-pinch into a stable "pencil beam" that can be propagated to the target. Pressures above 10 torr are inadmissible because of multiple scattering.

In summary, present results on both high power and beam focusing (that is, on the transport of ions to the talge! are rrouraging. A multiple-beam experiment is being built to test and refine these results. But what happens once the beam reaches the target?

\section{BEAM-TARGET INTERACTIONS}

The stopping of charged particles in matter has been studied for about 70 years. The simplest the rries that just calculate the energy transferred by bin.ry Coulomb collisions from the beam ions to the electrons in the stopping medium explain the principal feaiures of the beam-target interaction. Although agreement between theory and experiment is excellent at low intensities, it has not yet been possible to test the theories with fusionintensity beams. However, as binary Coulomb collisions must occur, even with intense beams, the range of ions in a fusion-interisitz bearn is unlikely to be substantially larger than calcusated. Thus the range of ions is not a major issue in the assessment of heacy-ion fusion. although more accurate range information will ultimately be required to optimize specific target designs. A more urgent issue is whether the beam-target interaction will generate particles or photons that excessively preheat the target fuel.

Typical high-gain targets with deuterium-tritium fuel can tolerate about $10^{5} \mathrm{~J} / \mathrm{g}$ of preheat energy in the fuel. For fusion, a target requires about $10^{9}$ joules of beam cnergy per gram of fuel. The mass of the fuel is usually 10 per cent of the total mass of the target. Thus, if the preheat is deposited uniformly throughout the target, preheat energy less than about 0.1 per cent of the total beam energy is acceptable. In the following discussion, we pessinistically assume that all energy deposition in the fuel occurs early enough to contribute to preheat.

Secondary particles or photons capable of penetrating deeper than the ion beam might be produced through the electromagnetic interaction as "knock-on" electrons anc ions, electrons from plasma instabilities, bremsstrahlung and $x$ rays. Likewise, the nuclear interaction migh generate such products as charged nuclear debris gamma rays, and neutrons. We will examine thes possibilities separately.

\section{Knock-On Electrons and lons}

These are secondary charged particles generated it elastic collisions between ions in the beam and electron or nuclei in the target. We can calculate the maximun kinetic energy that an electron could get from an ion in head-on, nonrelativistic collision. Then, using standar range-energy tables, we can show that the maximun electron range is always less than the range of the ion tha produced it. Thus knock-on electrons cannot generat preheat.

What about ions? When we examine the effect of th field of an ion in the beam a id compare the transfer $c$ kinetic energy from such an ion to either e!ectrons or ion in the plasma, we find that, because of the difference $i$ mass, the energy loss to ions is about $10^{-4}$ times lowe than it is for electrons. Thus, nearly all the beam energy transferred to electrons, but, as we saw above, their rang is too short to cause a preheat problem. On the othe hand, although knock-on ions will penetrate further. th maximum energy that they could get from the beam is a order of magnitude lower than 0.1 per cent of bear energy deemed acceptable for preheat. Thus, knock-o ions should not cause a problem.

\section{Electrons from Plasma Instabilities}

Some work has been done in this area but more needed. Generally, calculations show that if instabilitic do exist, they do not accelerate many electrons $t$ velocities substantially greater than the beam velocit: The electron velocity must exceed several times the bear velocity before the electron range exceeds the ion-bear range. Therefore, few electrons from plasma instabilitic could penetrate in to the fuel.

\section{Bremsstrahlung}

This radiation would be generated primarily fror knock-on electrons. The fraztion of electron energ 
radiated as bremsstrahlung depends on the energy of the clectrons and the atomic number of the target material. For typical electron energies and target materials, the bremsstrahlung is smaller than 0.1 per cent of the electron energy. Thus. bremsstrahlung is not a problem.

\section{Rays}

When the incident ion excites bound electrons. $x$ rays can be produced. By considering the $x$-ray absorption upacities of iypical materials in a target and making the outiageously pessimistic assumption that all the beam energy is converted into $x$ rays, we can calculate an upper limit on the energy absorbed per unit mass. For a typical target. the beam deposits about $10^{7} \mathrm{~J} / \mathrm{cm}^{2}$. Because thermal $x$ rays are copiously produced at energies of 1 $\mathrm{keV}$ or less and are usually accounted for in the target design. only $x$ rays in the 10 - to $100 \cdot \mathrm{keV}$ range need be considered for preheat. At these energies, the absorption opacity of the fuel is about $0.01 \mathrm{~cm}^{2} \mathrm{~g}$ so that only $10^{5}$ $\mathrm{J} g$ would be absorhed in the fuel if all the beam energy went into such $x$ ays. Therefore this source of preheat is unimportant.

\section{Charged Nuclear Debris}

Cross sections for nuclear reactions are never much larger than simple geometrical cross sections, which give mean free paths of 10 to $100 \mathrm{~g} \mathrm{~cm}^{2}$. Because the ranges of typical ions are $0.01100 .1 \mathrm{~g} \mathrm{~cm}^{2}-\mathrm{a}$ factor of 100 lower. only 1 per cent of the particles is involved in nuclear reactions that might generate charged nuclear debris. Detailed calculatuns show that preheat from this source is acceptable.

\section{Gamma Rays}

As in the case of energetic $x$ rays. the absorption opacity is so small for gamma rays (about $0.1 \mathrm{~cm}^{2}, \mathrm{~g}$ at a typical energy of $(\mathrm{MeV})$ that fuel preheat would not be a problem even if a!l the energy of the interacting nuciei were converted to gainma rays.

\section{Neutrons}

The mean free path of neutrons in deuterum-tritium fuel is severai grams per square centimeter. which cor- responds to ari effective absorption opacity of several tenths. Thus a calculation identical to that for gamma rays again gives acceptable preheat.

The tentative conclusion is that the interactions are compatible with the requirement: of ICF. ${ }^{8}$ Nevertheless. it seems prudent to test this conclusion before building a full-scale fusion accelerator.

Los Alamos and Lawrence Berkeley Laboratory plan to build a test accelerator capable of providing a definitive test of the beam-target interaction. ${ }^{4.10}$ This test accelerator and its associated experimental program are designated the high-temperature experiment. The completion of the accelerator is currently planned for 1989 or 1990.

The high-temperature experiment will not test just the beam-target interaction. It wiil also provide a facility for studying beam focusing. The high-temperature experi. ment, which will develop and test accelerator technology at a level beyond the multiple-beam experiment. will still not be large enough to produce significant fusion energy. It will provide engineering and cost daia that are ssential to a sound evaluation of the promise of heavy-ion fusion.

Present theory and experiments indicate that heavy-ion accelerators are well suited to inertial fusion power production. Although lasers and light-ion diodes can provide essential physics information in the near future. it is the author's opinion that heary-ion accelerators offer the best prospects for commercial energy production.

The multiple-heam and high-temperature experiments will go a long way toward determining if this opinion is correct.

\section{REFERENCES}

1. Lawrence M. Lidsky. "The Trouble with Fusion." Technology Revien' 86. 32-44 (October 1983).

2. “ERDA Summer Study of Heavy Ions for Inertial Fusion." R. O. Bangerter. W. B. Herrmannsfeldt. D. L. Judd. and L. Smith. Eds.. Lawrence Berk=loy Laboratory report LBL 5543 (December 1976).

3. R. O. Bangerter. J. W. K. Mark. and A. R. Thiessen. "Heary Ion Inertial Fusion: Initial Survey of Target Gain Versus Ion-Beam Parameters." Physics Le'ters $88 \mathrm{~A}, 225(1982)$.

4. W. Chupp. A. Faltens. E. C Hartwig. D. Keefe. C. H. Kim. C. Pike. S. S. Rosenblum, M. Tiefenback. 
D. Varecek, and A. I. Warwick, "A High Current Heavy Ion Beam Transport Experiment at LBL," Lawrence Berikeley Laboratory report LBL. 17256 (January : 984 ).

5. "HIBALL-A Conceptual Heavy lon Beam Driven Fusion Reactor Study." Vols. 1 and 2. University of Wisconsin report UWFDM-450: Kernforschungszentrum Karlsruhe report KfK-3202 (December 1981).

6. "Heavy Ion Fusion Reactor. HIBLIC I," Nagoya University, Nagoya 464. Japan, Institute of Plasma Physics report IPPJ-663 (January 1984).

-. C. L. Clson. "Final Transport in Gas and Plasma." in Proceedings of the Heavy Ion Fusion Workshop. Lawrence Berkeley Laboratory report LBL-10301:
Stunford Linear Accelerator Center report SLAC. PUB-2575 (September 1980), p. 403.

8. R. O. Bangerter. "Ion Beam Interactions with ICF Targets." in Laser Interaction and Relaced Plasma Phenumeno. Vol. VT. H. Hora and G. H. Miley, Eds. (Plenum Publishing Corporation, New York. 1984) pp. 1013-1027.

9. R. O. Bangerter, "Accelerator Inertial Fusion-A National Plan for the Development of Heavy-Ion Accelerators for Fusion Power," Los Alamos $\mathrm{Na}$ tional Laboratory document LA-UR-81-3730. Le. cember 1981.

10. R. O. Bangerter. "Heavy-Ion Fusion Accelerator Research Program Plan for FY84-FY89." Los Ala. mos National Laboratory document LA. L'R-83-1717. May 1983. 
ABSTRACTS OF ICF SUPPORTED

RESEARCFi PUBLICATIONS SINCE 1982

FXPERIMENTAL PLASMA PHYSICS AND ATOMIC PHYSICS

THEORETICAL PLASIA PHYSICS

SPACEPHYSICS

DRIVER TECHNOLOGY AND FACILITIES

MATERIALS TECHNOIOGY

ICF SYSTEM STLDIFS

PATENTS 
REPRINTS IN

EXPERIMENTAL

PLASMA PHYSICS

AND

ATOMIC PHYSICS

SINCE

1982 


\section{Abstract Subminted for the \\ 1985 IEE⿷ Intermational Conference on Plesma Sciตnco}

PLASHA ARHATURE haILGUN STUDIES" Los alamos, MA 87545
J.V. Parker, H.A. Parsons, C.E. Cueasnga, H.E. Fo Los Alago Matlonal Laboratory

Pittoburgh Hilton

June 3.5. 1985

It has deen nearly a docade ince the firot plesed argacure ralligun experiments at AHU. Desplto oxtonsl re Jevelopgent work ourlng the intervanelng yoars the plasma ardature ratlgur mas not demongtrated the nigh roloclty performanie whloh was gonercily anticlpated. In race, on $1 ;$; mandful of experlments have oxceeded the $5.9 \mathrm{~km} / \mathrm{g}$ reloctty achloved at aNU and tne A!gnest peported velocily 13 only $\| \mathrm{km} / \mathrm{s}$.

Jurbing the past year a detalleo invogtigation of Disal arazicure rallgun porformance has been carrind ou: as pare of the HYvaX program at Los Alamos. The priactpal resuliss of enis invesetgation are:

do!s:lan of macerial from the walls by the flasma armature is the post laportant ldatbation on rallgun performance.

2 Aolation sets an upper liati to the velocity whlet. can je acniayed for a given rall geometry and operating current. independent of projectile wass. glven oy

$$
v_{\max }=L^{\prime} l i z a v_{a}
$$

where is the ar = voltage an a. a proportionaliy constant relating are input power to mass ablation rate.

1. The surren: dig:-toution 17 the plagma argat ure varles rapljty wi:7 tate and posicton 30 ina: quas:-sta:l = Dodei, of the plasma arqature have linitec ut:itty.

- yea- Ene waxidum velocley the are zurront alstrtbution san become very complez. exnlolting offects suen as: separation from the project.le, dultipis at -rent peaks, and rapld deceloration.

The resul: sumpartized above are suppor:ed oy a :arze Dody or experimental daca taken on the HYVAX-1 a:-Bगन. HYYAX-: is a single stage, 11 an. dismeter pore Jevize witn an active :engtn of 2.75 eetars. The pewer supaly sogprlags three lindependent capacltor bank modules wnten can to sequenced to provlde a contrjlled current wavefors. Typlcal operating condi-

$\therefore$ s7s are; projectlle mass of 1.0 to 1.5 grams.

- jection veloctiy of 800 to $1900 \mathrm{~m} / \mathrm{s}$, poax eurrent of jo to $300 \mathrm{kA}$, and ina : veloclty of 2 to $4 \mathrm{~km} / \mathrm{s}$.

olagnosics include the uscal yolcage, current and sagretle prode measuroments. Significant foprovements in our understandihg of are behavior have resulted ron ualna magnetlo probes which senge the rill cur-

ront. Hecoralng the integrated output of thege prodes Erovides measure of the current floung in the ralls 1.: 6 to 9 positions along the barrel, thus provlding :oth temporal and spatial inforration about the plasas i.urrent alstribution.

Ongolng experlwents are address!ng the Bftect of re neteriats on bolation phenowena w:th the boal of igroved $h$ lgh veloc lity performance.

work perrorded under the auspless or the i.j. Des:. of Energy. subjext cotogury eno number Electrumagne: 'L Launchers 22 .

$\square$ Proler orsil session

$\square$ Preter bosier secsion

Do preference

Submitled by

\begin{tabular}{l} 
Jerald V. Parker \\
Los Alamos Nationa L Laboratory \\
\hline Group F-7, AS-E525 \\
Los Alamos, New Hexico B7545 \\
505) 667-3119
\end{tabular}

Iaportant

The centuretse Rreots all on opouceo of allect

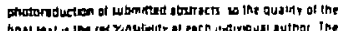

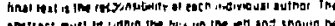

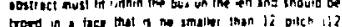

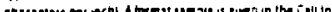

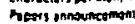




\title{
Evidence for Collisional Damping in High-Energy Raman-Scatterint
} Experiments at $0.26 \mu \mathrm{m}$

\author{
R. E. Turner. Kent Estabrook, R. L. Kauffman. D. R. Bach, (') R. P. Drake, D. W. Phillion. \\ B. F Lasinski. E. M. Campbell, W. L. Kruer, and E. A. Williams \\ Lawrence Litermore Valional haborator!', Universing of Calsformia. Livermare. Californu 04550 \\ (Received 28 Ausust !984)
}

\begin{abstract}
Experiments using i $j \mathrm{~kJ}$ of $0.26-\mu \mathrm{m}$ wavelength light to irradiate thin "burn through" larzets show less stimulated Raman scattering (SRS) than similar experiments at $0.53 \mu \mathrm{m}$. The SRS fiom high- $Z$ (Au) targets is $]$ orders of magnitude less than SRS from low- $Z$ (CH) targets irradiateu $:$ similar intensities. These resulis are the first direct observation of collisional damping of the Raman instabilisy in high- 2 argets us rredicted by theory and simulations.
\end{abstract}

PaCS n,mbers $5250 \mathrm{jm}$, i2 25 Rv, $5235 \mathrm{Pv}, 5240 \mathrm{Db}$

Raman scattering ${ }^{1-5}$ (SRS) in laser-produced plasmas can generate high-energy electrons, which can degrade laser-fusion targel gain. In nearly all previous laser-plasma experıments, SRS has been limited by steep density gradients, which restrict the phase. mdiching regions to relatively small length, However. the reactor-size targets envisioned for future laserfusiun experıments will produce much longer densitygradient lengiths. Here we report the otservation of an important diternative limiting mechanism, electron-ton collisions, which car inhibit SRS.

In these experiments. uv laser lir : I irradiaies thin films causing them to expand rapidly. By the peak of the 1-nsec laser pulse, the electron density is near resonance $\left(n_{c}, 4\right)$ for the Raman ${ }^{j}$ and $2 \omega_{p e}{ }^{b}$ instabilities. Other :nstabilities that can produce hot electrons occur near crillcal densily $i n_{c}=10^{21}[11.06 \mu \mathrm{m}) /\left.\mathrm{A}_{0}\right|^{2} \mathrm{e}^{-}$, $\therefore \mathrm{m}^{\prime \prime}$ and are thus mostly excluded from these observatuons. With plas $(\mathrm{CH})$ largets, the fraction of the lacident enerey $\mathrm{sc}_{\mathrm{a}}$ red as Raman light is $S$ times imaller for irradiations with a laser wavelength $\left(\lambda_{n}\right)$ of $026 \mu \mathrm{m}$ than for $0.53 \mu \mathrm{m}$. With Au targets, the decrease is more than 4 orders of magnitude. We use theory und simulations to show that the suppression of SRS with increasing laser frequency or target $Z$ is due to colisional damping of SRS.

These experments used one $4 \mathrm{rm}$ of the $74-\mathrm{cm}$. aperture vinvelle laser An array of polassium dihy. drogen phosphate crystals quadrupled the frequency of the $105-\mu \mathrm{m}$ laser light to producs : $\mathrm{p}$ to $15 \mathrm{~kJ}$ of light dt $0.26-\mu \mathrm{m}$ wavelength in a near Gaussian pulse of $09-! 2$ isec full width at half maximum . FWHMI) A r ask at the senter of the lens and the chromatic aberration of the : 1 focusing lens caused the uv light to focus in a region frec $[\mathrm{r} \cdot \mathrm{m}$ lesidual $1.05-\mu \mathrm{m}$ and

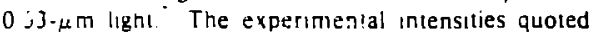
here are the average laser power divided by the 11iumınated drea is determined from $x$-ray micrographs (x-ray cnerg: of $-1-i \leq \mathrm{keV}$ ) We define the beam diameter. $D$. as ine FWHM of the $x$-ray intensity flthough the haser beam is known to be nonuriform." dad qudliturely less unilorm than the beam at 053 $\mu \mathrm{m}$. iocalized regions of higher intensilies should enhance, not reduce, the growth of Raman scattering.

The targets were $6-\mu \mathrm{m}$-thick $\mathrm{CH}$ or $0.4-\mu \mathrm{m}$-thick Au folls, supported on large washers. The thicknesses were chosen, by LASNEX calculations. so that the max. imum eiectron density on-axis would be alightly less than $n_{\mathrm{f}} / 4$ by the peak of the laser pulse. The parabolic density scale lengtios are calculated to be $1500-$ $1000) \lambda_{0}$ in both the axial and radial directions for both $\mathrm{CH}$ and $\mathrm{Au}$. The targets were irradiated at normal incidence, with the focus in front of the target. The focal-spot diameters were 300 to $350 \mu \mathrm{m}$, resulting in average target-plane intensities of $(1-2) \times 10^{15} \mathrm{~W} / \mathrm{cm}^{2}$

A streak camera and optical system, nounted orthogonal to the laser beam. provided time-resolved one-dimensional spatial images along the direction of the laser axis. ${ }^{10}$ This instrument looked at the $313 \cdot \mathrm{nm}$ bremsstrahiung emission from both the front and the back expanding plasmas. When the plasma is optically thin (lou- $Z$ green exper nts). this emission is proportional to the line-averaged product of the densily squared and the square root of the temperatire. The simulation line integrais are in good agreement. A ume-resolves opucal spectrometer measured the Raman lighi scattered at $135^{\circ}$ to the jaser beam. Calibral. ed pho'odiodes, filtered to look only at SRS from below $\pi_{\mathrm{e}} / 4\left(0.35 \mu \mathrm{m}<\lambda_{1}<0.5 \mu \mathrm{m}\right)$. measu:ed the absolute level of Raman-scattered ligh: at various an. gles (between $105^{\circ}$ and $169^{\circ}$ from the lajer) Spec tre scopy verified that all Raman light emissions iters within this range of wavelengths.

The results are summarized in Fig. I The error bars enclose ail the data obtanned during the experiments. We estimate the absolute accuracy to be within a factor of 2; the principal source of error arises from the finite number $(10)$ of photodiodes and the result. ing uncertainly in the true angular distribition of the scattered lighil. The data analysis is identical to that discussed by Drake et al. ${ }^{11}$ Most of the SRS energy was within $45^{\circ}$ of direct backscatter, althouph no measurements were made back !hrough the $f 4$ lens

Figure $l$ also shows the data $f i c m$ previous $0 \leq j . \mu \mathrm{m}$ 


\title{
Meastrement and Analysis of Near-Classical Thermal Transport in One-Micton Laser-Irradiated Spherical Plasmas
}

\author{
A. Hauer, W. C. Mead, and O. Willi \\ Los Alamos Nasional Laboratory, Los Alamos. Vew Mexico 87545 \\ and \\ J. D. Kilkenny, D. K. Bradley, and S. D. Tabatabaeı \\ Imperial College, London, Unted Kingdom \\ and \\ C. Hooker \\ Rutherford Appleton Laboratory. Chulon. Didcot. United Kingdom \\ (Received io Augusi 1984 )
}

\begin{abstract}
Solid spherical layered targets have been unifortrily illuminated at irradiances of $10^{14} 10$ $10^{15} \mathrm{~W} / \mathrm{cm}^{2}$ Extensive diagnostics including tume-resolved $x-r a y$ emission and optical prob ing were used to determine the plasma ablation rate and the plasma blowoff conditions Comparisons with hydrodvnamic simulations show that the thermal conducion is well characterized by a nux limit $f_{e}=0,08 \pm 0.02$. with a steep temperature gradient The steepness of the heat front was confirmed with tume-tesolved spectroscopy
\end{abstract}

PACS numbers. 52.25.Fi. 52.50.Jm

Knowledge of energy transport from the region of the laser-light absorption to denser portions of laser-produced plasmas is crucial to an understanding of the laser-induced ablation process. ${ }^{i-3}$ Extensive recent computer simulation work ${ }^{3}$ has led to the expectation that "classical" transport in spherical laser-heated plasmas can generally be approximated by flux-limited diffusion with a flux limiter in the range of $f_{e}=0.06-0.2$. Early work, largely involving flat targ ts, indicated strong to moderate inhibition of the heat flux relative to classical predictions." Some of the more recent work involving spherical targets has indicated transport levels closer to classical, ${ }^{5}$ with evidence of a penetrating foot in one case. ${ }^{6}$ Other work indicates some nux inhibition even in spherical geometry.

In the present experiment we have used a comprehensive set of diagnostics to characterize the plasma conditions and thermal transport in a spherıcally symmetric plasma. We have made timeresolved spectroscopic measurements (using both low- and moderate- $Z$ radiating ions) of the penetration of the heat front in spherical plasmas. In addition, we have used optical probing to measure simultaneously the density profile of, and the mag. netic field in, the underdense plasma. Comparisons with hydrodynamic modeling show that (a) all observables are consistent with a high $\eta_{1}$, limit and (b) the heat front shows ..o observable it.

The targets used in this study consisted of solid glass spheres $1-160 \mu \mathrm{m}$ diameter 1 , containing $S$ and $\mathrm{Ca}$ (among other consttuents), coated with three tayers: (1) $\mathrm{CH}(0.5$ to $2.5 \mu \mathrm{m}$ (hick). (2) $\mathrm{Ai}$ $(0.1 \mu \mathrm{m})$, and (3) $\mathrm{CH}(0.5$ to $2.5 \mu \mathrm{m})$. The solid glass ball prevented implosion of the target (and subsequent inward movement of the critical surface).

The targets were uniformly illuminated with the six-beam, $1.06-\mu \mathrm{m}$, laser facility at the Rutherford Appleton Laboratory. The six beams were focused by $f / 1$ lenses with optical axes along the faces of a cube. Incident irradiance levels were in the range $(1.5-15) \times 10^{14} \mathrm{~W} / \mathrm{cm}^{2}$. The laser pulse had an approximately Gaussian temporal profile with 0.83-ns full width at half maximum (FWHM).

The radial intensity profiles of the beams were measured. Small-scale structure results in about $30 \%$ rms variation in intensity. The overlap of the six beams and beam-to-beam energy variation resulted in about $50 \%$ (peak-lo-valley) large-scale "ariation is incident irradiance across the surface of the sphere.

The primary diagnostic of thermal transport was time-resolved $x$-ray line spectroscopy. $X$-ray line emission was observed first from the thin alum!num layer (sensing temperatures of 200-350 eV) and later from the silicon $(-250-400 \mathrm{eV})$ and cal. cium $(-500-700 \mathrm{eV})$ in the glass ball. ${ }^{10}$

$X$-ray emission from the target was dispersed (with a thallium acid phthalate crystal) onto the sitt of a streak camera covering 17 to $2.4 \mathrm{eV}$ The spectral and temporal resolutions were about 500 $(\mathrm{A} / \mathrm{\Delta A})$ and $50 \mathrm{ps}$. respectively. A seventh laser beam with very short duration $i-100$ ps was used 
From: LASER INTERACTION AND RELATED PLASMA PHENOMENA, VOl. 6 Edited by Heinrich Hora and George H. Miley (Plenum Publishing Corporation, 1984)

\section{SUPRATHERMAL ELECTRON GENERATION TRANSPORT AND OEPOSITION IN}

\section{$\mathrm{CO}_{2}$ LASER IRRADIATED TARGETS}

All lan Hauer, R. Goldman. R. Kristal, M. A. Yates, $M$. Mueller, F. Begay, D. van Hulsteyn, K. Mitcheil. J. Kephart, H. Dona, E. Stover, J. Brackb111, and D. Forslund

Los Alamos National Laboratory University of California Los Alanos, $\mathrm{M}$

\section{ABSTRACT}

In $\mathrm{CO}_{2}$ laser interaction with matter most of the absorbed energy is initially channeled into a hot electron distribution. In many cases, resonance absorption is thought to be the dominant mechanism producing this distribution. Stimulated scattering may also play an important role.

In the coronal region of the laser plasma, hot electrons suffer losses that fall into two basic categories. First, hot electron energy is used in the sheath to accelerate fast ions. In some cases this can be a very efficient process. This is an important interaction, since some ICF concepts use energet ic ions as the drive mechanism. The other coronal loss mechanism is the loss of energy to cold electrons through the orawing of a return current. Some aspects of the absorption and coronal loss processes will be illustrated by experiments on aser irradiated shelis.

Experiments on both axial and lateral energy transport and deposition in spherical targets are described. A variety of diagnostics have been used to measure hot electron transport and deposition including bremstrahlung and inner shell radiation and soft $x-r a y$ temperature measurements. Self-generated electric and magnetic fields play an important role in the transport and deposition of the hot electrons. In some cases distinct patterns of surface deposition consistent with magnetic field configurations have been observed. 


\title{
Determination of laser intensity and hot-electron temperature from fastest lon velocity measurement on laser-produced plasma
}

\author{
T. H. Tan, G. H. McCall, and A. H. Williams \\ Unuersiry of California, Las Alamos Nazonal Laboratory. Las Alamas. New Mexico 8 is45
}

(Received 8 Jure 1982; accepted I August 1983)

\begin{abstract}
Measuremens of the velociny of the fastest ion emitted by a $\mathrm{CO}$-laser-produced plasma were made, and it was found that the relationship between velocity and laser flux was given by $\boldsymbol{V}$. $=5.7 \times 10^{\circ} \omega_{i}^{\prime a}$ for singie-beam illumination. Comparison with computer simulation, ion velocity spectrum, and $x$-ray data indicate that the hot-electron temperature is given by $T_{\text {* }}$ $=7.5 \times 10^{-1} V^{\prime 2}$. Measurements of emission from multuple-beam experiments and from expenments at i.06 $\mu \mathrm{m}$ wavelength sre aiso descrited. Some simple modets are used for discussion and correlation of the data
\end{abstract}

\section{INTRODUCTION}

When a high-intensity laser beam interatts with matter electrons play a primary role in absorpion of hight and in energy transport The hot-cleitron distribution which re sults from the interaction depends on the laser pulse shape. power, focusing conditions, and wavelength, and therefore the measurement of hot-electron temperature provides im. portant information about the interation dynamis or the laser focusing and pulse quality Determination of hot-elec tron temperature by measuring the slope of the high-energ: $x$-ray continuum spectrum: has been i generally accepted technique for low-iaser-intensatv anter-cition. Al high inten suty, however. the presence of substantial background nome and the instrumental limitation in enctgy discrinination ian ufiect the accuracy of actual x-ray eneryy spectrum defer. mination. In addition. the copious emission of fast iuns from the laser-produced plasma con : : stes the currelation of the $\checkmark$-ray spectrum with the actual source hot-electron distribu ton. On the other hand. weause of the collective behaviur il plasma. the motion if the .rot electrons can generate an ex. tremely high ste:trostatic potential which tesults in the emision of uliratist ians " Therefore. We can expect the fast-ion expansion to correjate tlosely with the hot-electron distributiun. Thus, the study of iast ons provides unother means to probe quantmatively the hot electrons generated in the plasmd In this paper. we shall demonsirate expenmen-

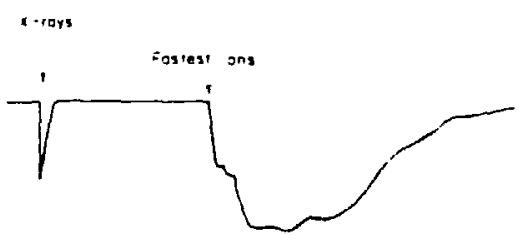

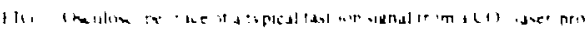

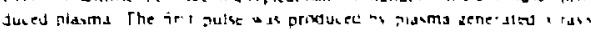

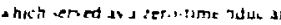

lally that the fastest - inn velncity does, in fact. uniquely correlate with single-beam laser fux on target, and that by an empirical fit and an analytical model. the temperature $i$. that tharat:azes the hot-elettron energy distributson :ant also be inferred Relevant p-ray measurements. Hielength waling, and the effects of multibeam illumination on hot. electron distribution determination have been examined and will be included in the discussion.

\section{FASTEST ION VELOCITY MEASUREMENT AND LASER INTENSITY ON TARGET}

Figure i shows an oscilloscope trace of a is pical fast - 16n "gnal from a $\mathrm{CO}$--laser-produced plasma messured with scintillator-photomulaplier detector ${ }^{*}$ which can detect a ingle son. Many such detectors have been fieided. and their Jistances trom the target range from 15 to $\mathrm{J} m$. The a ming ipeed of the oscilloscope is set between 10 to 100 nsec per division The first pulse is priduced by $y$ rays generzied in the piasma and is useiul as a zero-time fiductal. The fastnsing ton signal is charactenstic of a plasma produced hi a last-nsing laser pulse. and the nse tume cun he ust $d$ as a laser diagnostic If the mann laser pulse is preceded by a low-intensitv precustor, such as from I prepulse or prelase. the ron ugnas nse tume is significantl longer than for the case shoun in Fig. 1. probably hecause of a chang. in plasma wale length. The sharp nse also shows that there is a maxumum un selocity.

Figure a shciws the distributiun of the fastest-ton seloc 11) 1 . 5 a function of 'aser intensity for flat and spherical targets llfuminited by a s ngie beam ar $10,0,4 \mathrm{~m}$ warelengl.̈ wing three laser svistems at the Los Alamos Niatlonal Laboratory SES. a single-beam prototype: Gemini, a iwo-beam prototype, and Helios: Each system uverlapped in in ensity and pulse length with the next-ingher energy sysiem. Energy in target ranged from 0.5 to $1000 \mathrm{j}$. and pulse langrhs , FW H.M ranged from 00 to 1 o nsec. Focal spot diameters ranged from 71 to $130 \mu \mathrm{m}$. The data, shown it. Fig 2, were wont rolled samples where the beam spor dar . which en. circled $30 \%$ of incident iaser energ: was T...... red at the 


\section{Retura-Current Heating and Implosion of Cylladrical $\mathrm{CO}_{2}$-Laser-Driven Targets}

A. Hiner and R. J. Meron

Los Alamos Natlonal Laboratory. Umbersity of Calyomin. Los Alamos, New Menico 87545

(Aecelved 1 Aprll 19s3)

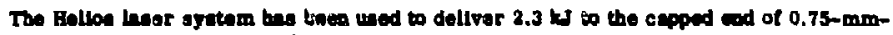

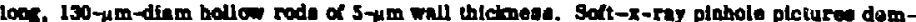

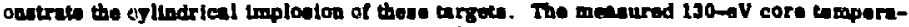

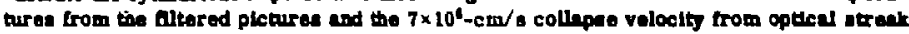

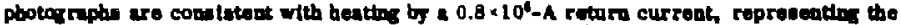
recyalins ef 159 of the hot-electron eminaton.

PACS mumbers: $52.50 .3 \mathrm{~m}, 52.25 . \mathrm{n}, 52.30 .+r, 52.70 .-m$

At intensities which exceed $10^{16} \mathrm{~W} / \mathrm{cm}^{2}$ most of the energy aboorbed trom $\mathrm{CO}_{3}$ lagera goes dlrectly into a relatively amall number of highly energetdc (> $200 \mathrm{keV}$ ) suprathermal electrons.' Tha energy must be traniferred to a much larger number of localized thermal electrons to accompliah laser tusion. Since direct cisasical coupling of the suprathermals to the backrsound plasma has proven inefflcient, we have initlated an effort to use the gelf-generated fields in speclalized targets to improve the energy tranafer. Charge imbalance develops as auprathermals leave a laser spot. The resultant $E$ flelda can draw a return current, $L^{*}$ an appropriate path is provided. Benjamin et al." have demonstrated the existence of these currents, and their ability to heat a target support stalk. This Letter reports the first experimantal resulta from targets dejigned to use the return currents to heat and implode a thin-walled hollow cylinder $\rightarrow$ procuce a micro $Z$ pinch.

A scnematic of the "augmented return current" (ARC) targets used in the experiments is given in Fig. 1. Four tightly foused $\mathrm{CO}_{2}$-1ager benms from the Los Alamos Helios laser gystem im pinge on the capped end of a long hollow, low $-Z$ cylims: $a$, coated with a thun layer of metal (gold or alumirum). The cylinder is mounted at the center of a large high- $Z$ disk. Suprathermals generated at the locus will drift toward the disk in the plasma surrounding the cyilnder. A thermal return current is drawn back along ita walls. Resistivity of the wails results in Joule heating of the thermals. The $B$ field from the resuitant current loop can implode and further heat the cylinder.

Experiments were performed on targets with a variety of diameters and lengths. The smallest cylinders were matched to the minumal laser spot diameter. Their length was set at roughly 5 dianieters to maximize the aspect ratio for cur- rent-related effects, under the constraint of minimum mans- for madmum temperature gain from energy deposition. The minimum wall thickness was set by limits on structural integrity. The metal layer was added to provide 2 brighter signature in $x-r$ ay pinhole pleturea. The eartier experiments' recorded currents from eiectrons escaping to the walls of the target chamber. The ARC targets were designed to augment thls current through the axial allgnment of the cylinder and beams, with a cylinder much thicker than the earlier 10- $\mathrm{mm}$ atalke, and with the addition of the collector disk for poesible suprathermal entrainment. The presence of thermoelectric, $\nabla n \times \nabla T B$ flelds has been recogaized for gome time." The cylindrical return currents axd any pinch effect should serve to enhance these flelds. Suprathermal drift down the cylinder ${ }^{7}$ is consistent with a $B$ fleld, drected as in FIg. 1, and with an outwardly directed $E$ theld for containment of the electron cloud. The axial $E$-fleld component, drawing the return cur rent against resistivity, introduces a "tilt" in the $E \times B$ drift, which should aid capture of the suprathermals by the cylinder wall. Alternative$1 y$, the $B$ field a round the cylinder should help to shield its intertor Irom suprathermal preheat." The return-current effects discusged here will be reduced to the extent that deposited energy is lost to fast-ion blowoff. However, cylindrical geometry may serve to reduce fast-ion losses. as compared to those from foils, since the area

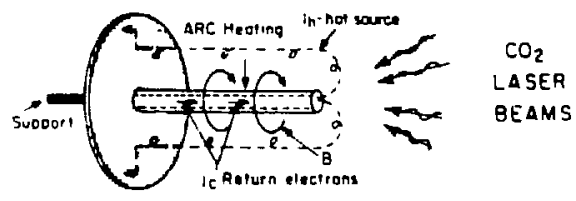

FIC. 1. Sketch of the ARI inget lestgn. 


\title{
Detailed observation and analysis of radiation from bigh-density leser-imploded urgets
}

\author{
Allan Hauer \\ Las dlamos Vational Laboratory, University of California, Las Alames, New Mexico d7sas
}

K. G. Whitney, P. C. Kepple, and J. Davis

U.S. Vaval Resuarth Laborator, Woshungton, D.C. $20 \mathrm{j}^{79}$

IRenved 22 November 1982)

\begin{abstract}
High-aneray $\mathrm{CO}_{r}$ iser radiation lis beers used to implocie ges-filled sphencal tarsets to very high sersitue $\left(10^{23}<n_{1}<10^{24} \mathrm{~cm}^{-1}\right)$ and temperature $(-800 \mathrm{eV})$. The imploded ges was compoead primanly of DT with a small amount of argon sed. The detailed observation of radiation from the ar. gon was a promary diagnostic of the imploded-plasma contitions. An extensive analysis of the emit. ted argon specinum provides a nch source of information on pissma parameters. It most cases one could not csume local-thermosvnsmic-equilibrium conditions. A very detailed collisionalradiative model aas constructed in order to analyze the spectrum. In view of the complexity of the calculations thm had to be periormed and the care needed in data acquisition and procr $\mathrm{ng}$. the
\end{abstract} sgreement between theory and expenment was very good.

\section{INTRODUCTION}

Lascr-dnven implosions have produced high-tem perature compressed plasmas with densities comparable 'o or greater than ordinary solids ta few $\left.\mathrm{g} / \mathrm{cm}^{3}\right)^{1-1^{1}}$ A whole new regime of very high-density, high-temperature plasma conditions has thus been opened for laboratory invesugaunon

The production of very high-density comprissed plasmas is central to the inertial-confinement fusion concep: Analysis of the radiation from theoe dense plasmas is an imporiant source of information on their condirtons. This analysis mas. however, involve relatively complicated modelung techntque In this paper He descnic techniques that have proved useful in the modeling of radiation from compressed plasmas with a density of $\perp$ [ew $\mathrm{gm} / \mathrm{cm}$ " The exfenment anaived here has been repored previuusly:

in $F_{1} g$. We show a ivpical target usad in high compression laser-implosion experiments. The targets are

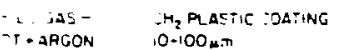

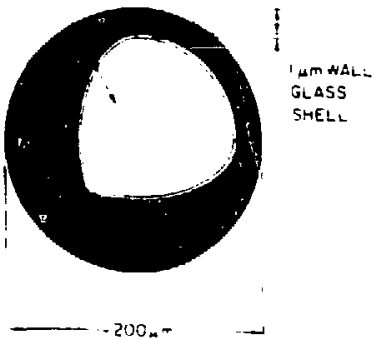

FlG i Target uxed lu produce high-Jensily taver-shven im. ploxions imploded by irradiating them with high-intensity $\mathrm{CO}_{2}$. laser light from the Los Alamos National Laboraton Helios laser facility. Laser energy on target ranged from $3-6 \mathrm{~kJ}$ and the half width of the pulec was $600-800 \mathrm{ps}$

Within the uarge: is a glass shell filled with a variety of gases; DT is used as the fuel for thermonuclear bum. Spectroscopic diagnosis of plasma temperature and dens1. ty is accomplished by seeding the DT fuel with a small amount of higher $Z$ material, such as nern or argon. Analysis of line emission from the higher $Z$ matenal is one of the most direct Jiagnostics of cumpressed densily.

The thick layer of plastic surrounding the gisss shell reduces the preheat of the fuel due to suprathermal $1-100$ keV/ electrons and allows an ablative implosion of the thel! Ir: earlier work of this type ${ }^{4}$ only the thin glass shell was present. Long-men-\{ree-path electrons tended to uniformly heat and thus explode this sthell. In the present work a slower less explosive implosion' has produced considerabiy higher densities of the order of 20 umes the liquid density

in Fig. 2, we show the general features of argon emis. sion from highly compressed plasmas. The top trace was taken with an exploding pusher target irradiated by about $35 . \mathrm{kJ}$ laser energy on target and represented a compressed electron density of about $9 \times 10^{22} \mathrm{~cm}^{-1}$ the muddle trace was ubtaned from a target with a coating of $25-\mu \mathrm{m}$ $\mathrm{CH}_{2}$, and $4.5-\mathrm{kJ}$ laser energy on target). The armpressed electron densily here is about $t \times 10^{24} \mathrm{~cm}^{-1}$ The final trace wis produced by a larget with $50-\mu \mathrm{m}$ $\left.\mathrm{CH}_{2}\right)_{8}$ coatıng and about $5.8 \mathrm{~kJ}$ of laser energy. The compressed electron tensity is about $7 \& 10^{23} \mathrm{~cm}^{-!}$In the experiment represented by the top trace of Fig. 2 the laser heats the glass shell directly; lines due to calcium and polasstum, which are impunties in the glass, are excited. When a laver of about $25 \mu \mathrm{m}$ of plastic is added these ines are suppressed.

The densities in these expenments: were measured pn manly by fitting tume integrated x-ray speciral profiles to theoretical calculations. The theoretisal calculations were made for specific densittes and did not attemipt to take 


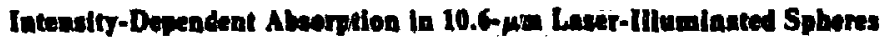

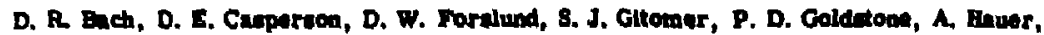

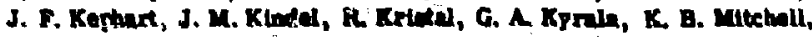
D. B. ven Boleters, and A F. millaris

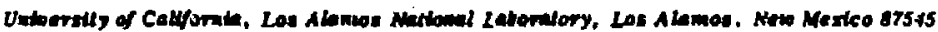

\section{QReoetved 7 raich 106)}

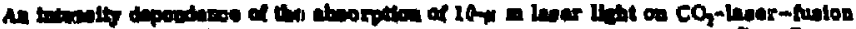

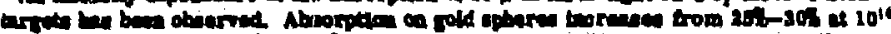

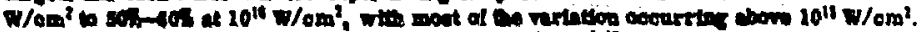

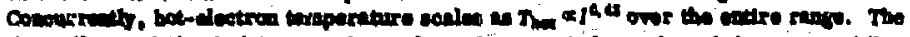

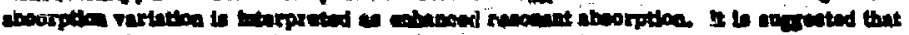

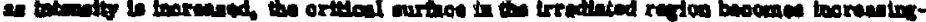

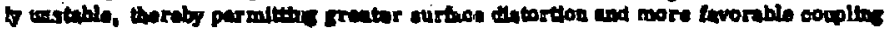
condition bor revones aborpiton.
\end{abstract}

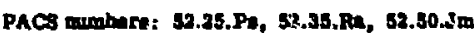

The absorption of laser enercy is in important factor in the overnll performance of a lever-fhsion larget eapoulc. At the 10.8- $\mu \mathrm{m}$ wavelength produced by $\mathrm{CO}_{3}$ inera, spherical-karzet as eorption van previously shown to be in the range $20 \%$ 30 for intenatties $\leq 10^{14} \mathrm{~W} / \mathrm{cm}^{2}$ and belew. 42 Wo report hart the chaervation of an increase in absorption to as bigh as $60 \%$ when intensity is Ineroaced to $10^{16} \mathrm{~W} / \mathrm{cm}^{2}$.

The experiments reported hare were done on the Los Alamos Natlongl Labroratory eight-beam Helios $\mathrm{CO}_{2}$ laner facility, at energiee up to $5 \mathrm{~kJ}$. The targets were gold spheres mounted on slender glase stalks. The dlameters were $0.3,1$, and $a \mathrm{~mm}$, with wall thickness betweer 0.3 and 31 $\mathrm{m}$. Absorption was measured with an array

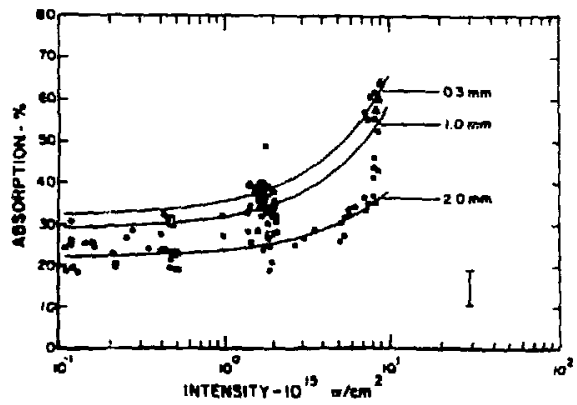

IT. I. Abnosptoe ve theneity for old epheres: trimglee, $0.3 \mathrm{~mm}$ dinm $(9$ ahots); cqunes, $1.0 \mathrm{~mm}$ Ham (92 bhots); dota, $2.0 \mathrm{~mm}$ dien (45 hot). Error

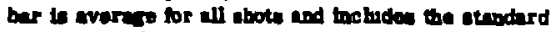
devintion of the variowe enbrimeters. The curves ero levt-Jquares liser ata to the data. of tourten calorimetern" deployed around the target chamber, ench sensitiva to particle nox

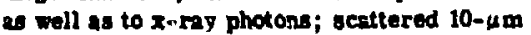
light is apecliteally rojected.

Figure 1 shewe the absorption as a function of indenolty. The latter is maried primarly by delocuping and ocensionally by total-eneres variation. Indtridual beam intencity at beet locus is computed with a $100-\mu \mathrm{m}$ spot diameter containing 75\% of the energy, and a pulse witth of 0.75 as (tull with at hall maximum). For all elght beams, an energy-rreighted average is used to determine laser intensily. Defocused intenalty is cbtained Irom simple geometric considerations with an $f / 2.4$ beam expansion 1 rom the waist.

The data show a very clear doubling in absosption for 0.3- and 1-mm-diam spheres as intensity is increased from $10^{13}$ to $10^{10} \mathrm{~W} / \mathrm{cm}^{2}$. The effect is not as dramatic with the 2-mm spheres, absorption increasing from $25 \%$ to only $-35 \%$ at $10^{16} \mathrm{~W} / \mathrm{cm}^{2}$. The variation with target diameter may be an instrumental effect. Measurements of the ton anguiar distribution on flat targets ghow the presence of an ton plume in the direction of the target normal. In the present experiments the normal is nominally toward the laser focusing mirrors. The lons involved are not collected by the calorimeter system and may represent an increasingly signiflcant amount of undetected absorbed energy as the target radius increases. However, we cannct rule out the possibility that the diameter variation is real, a poist which we will return to later.

Additional supporting evidence for the intensity variation has been obtained from scattered-light measurements under single-beam illumination on 1-mm-diam targets. The $/ / 2.4$ focusing optics 


\title{
Use of Laue-geometry $x$-ray diffraction in the spectrosicopy of laser plasmas
}

\author{
Allan Hauer

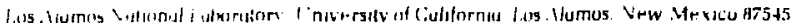

Racisud Mlurch in 14H.1

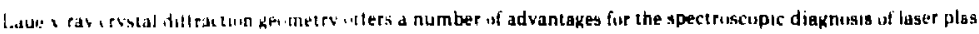

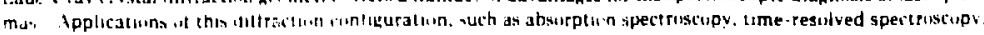

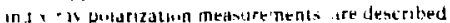

Crystal $x$-rav spectruscopy has become a commun and useful diagnustic of laser-produced plasmas.' Most of this wur! has utilized what is termed Bragg-diffraction geornetiy. in which the $x$-ray-diffacting planes are urproximately parallel to the entrance surface. Laue ceometry utulizes diffraction irrm planes that are approximately perpendicular to the entrance surface.The most important aspect of thas geometry is that it produces quasi-focusing, as shown in Fig. lla). All wavelengths from a point surrce are diffracted to lie (approximately) along the same line perpendicular to the axis that passes through the source point and is parailel to the diffracting planes.

The focusing is not completely stigmatic for either pertect or mosaic crystals. As is shown in Fig. $1($ b) the uidth of the diffracted bean is soverned by the thick. ness of the crystal." The width of the diffracted beams, unc' thus the approximate focusing resiolution, is

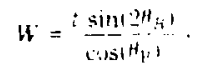

where $t$ is the crustal $:$ thickntss and $H_{H}$ is the Brags angle Given his limblation on the spatial resolution. the yuast-focusmn characteristic is still quite useful in spectrograph desigri Kinematic diffraction theory thow: that the maximum diffracted intensity in the Laue vermetry incurs when the orysial's thickness is I $u_{1 .,}$ where $u_{1}$ is ine linear ahsorption cuelficlent. A rrugh expressun tor the resolutan could thus be writ ten as

$$
H=\frac{\sin 12 H_{i}}{\mu_{11} \cos H_{H}} .
$$

A high energy spectrograph utilizing this geometry has already been uperated. ${ }^{.}$in this paper several uther Ideas are described for applications of Laue geometrs diffractiun to laser plasma spectroscipy.

Une application of laue diffraction is in atsorption -pectruscupy. The dense cumpressed one of laser. impluded piasmas mat sometimes he $t(w)$ coul to pro. dusce usable self-emissinn te.e. for dag tusis of com pre-sed densify. (lne methud of didgmusng such

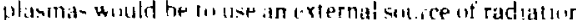

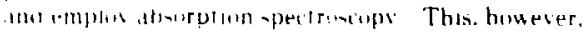

puses some problems for instrumentation. One wants to probe a region of small spatial extent. Crystal dif. fraction. however, requires an angular spread (given by Bragg's lawl in order to produce dispersion. In Fig. 2. we show two Laue diffraction configurations that are applicalis to absorption spectroscopy. If the source is small, the configuration of Fig. 2(a) is appropriate. In Fig. 2(a) diffraction from the second crystal is used to protect the film from being blackened by direct radiatiun from the main target. 'This configuration requires the rather precise alignment that is characteristic of double-crystal spectroscopy.2 A amall source may be more appropritute for strong high-energy continuum generation, since high laser flux will lead to higher plasma temperatures. If a more distributed source of $x$ rays is employed, the configuration in Fig. $2(b)$ is apprupriate. The quasi-focusing geometry will efficiently shield against barkground radiation (sucin as hard $\mathrm{x}$ rays generated by the primary target itself).

We consider a typical example of the application of the configuration in Fig. 2(b). For a moderately dense compressed core. probing with radiation in the region if $10 \mathrm{keV}$ might be appropriate. Consider a band from 10 tu $12 \mathrm{keV}$. For a Ge crystal $(220$ planes and $2 d=40$ A), the Brage angle would vary from 18 to $15 \mathrm{deg}$. In practice, the probe source cannot be located closer than

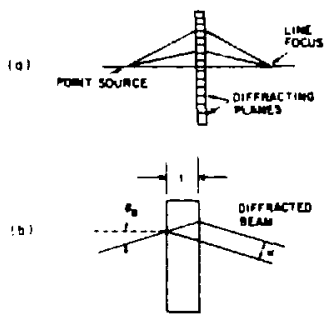

Fig 1. la Quasi incusing produced by Laue-geimetry dif tritern; iut if the plane of this tigure the heam is diverging The fine ixus is nurmal tu the plane ot the firure it, Illus. I ratun wh the ellect of cristal thukness in tocusing.

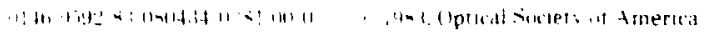




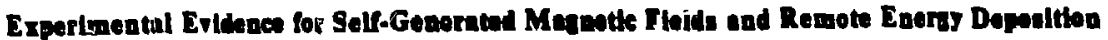 In Laser-Imadlated Taryots
}

M. A. Yates, D. B. ma Hulgteyn, H. Rutkowski, G. Kyrala, and J. U. Brachill Las Alamos Nattonal Laboratory, Los Alamos. New Mexico 87545

(Recetved 1 July 1982)

\begin{abstract}
In a serlas of expertments uatrg nwo-, four-, and olght-beam $14,6-4$ m-leser trradlation of a varioty of target geometries, a significant amount of eners we found to be depoulted in regtons cemote from the focal spots. The deponttion patteran can be predicted with a self-generated ruguetic field model.

PACS aumbers: $52.50 . \mathrm{Jm}$
\end{abstract}

Lateral trangport of energy away from laser focal spots can play an important role in redistributing energy deposition in laser-fusion targets. Work has been reported investigating the qualitative ${ }^{4}$ and quantitative ${ }^{2}$ mature of this transport. Recently, uaing a plasma simulation, Forslund and Brackblu' ${ }^{3}$ have Identified convective transport of electrons in self -generated magnetic tields as an important mechanism for surface transport in laser-irradiated toils. In one simulation with a laser intensity of $5 \times 10^{1 \mathrm{~J}} \mathrm{~W} / \mathrm{cm}^{2}$ in a 60- $\mu \mathrm{m}$ spot and a hot-electron temperature of 20 keV, peak fields of the order of 1 MG were calculated. The calculation has not been performed at higher intensities comparable to those used in experiment $\left(-10^{10} \mathrm{~W} / \mathrm{cm}^{2}\right)$ because the code does not handle the relativistic effects of the high-energy electrons generated at these intensities. In general, the ratio of electron to magnetic field pressure is of order 1 is a magnetized sheath whose thickness is large compared with the electron gyroradius. This Letter presents experimental evidence for the nonunitormity cf energy deposition predicted by the magnetic field model in a variety of target geometries progressing from nat, to cylinders to spherfa.

From the simulations, a simple qualitative moddel has been developed. Briefly, the model describes lateral energy transport by electrons in magnetic fields generated at the periphery of the laser spot by lateral temperature gradients in the corona. These gradients are maintained by electrons confined and drifting in the magnetic field, resulting in the convective transport of energy from the beam spot to the edge of the magnetized regi y. The interaction of the magnetic field and electrons produces a ther mal magnetic wave' which propagates across the surface until disrupted by fringing fields at the sarget edge or by destructive interference with the wave propagating from an adjacent beam.

1702
A result of this description is that the higherenergy electrons which transport energy away from the laser spot are magnetically insulated from the target surface. However, where thermal magnetic waves from adjacent beams interfere, as depicted in Fig. I(a), there is a magnetic field null. At that point the electrons deposit their energy into the target. We hnve performed an experiment with two beams on a tlat target which dramatically shows this effect, as seen in Fig. 1(b). In addition, we have taken data in more complex geomelries which also show patterns of deposition consistent with transpori tominated by self-generated tields.

All experiments were performed at the Hellos laser facility" of the Los Alamos National Laboratory. This is a carbon-dioxide system with a

(a)

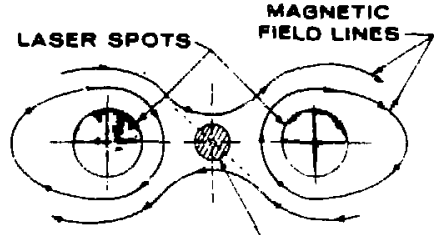

(b)

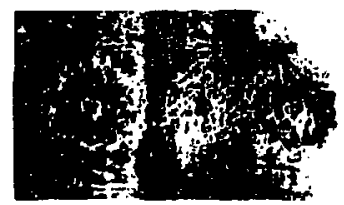

FIG. 1. Energy deposition region between two laser beams as (a) predicted by slmulations using convective electron transport in self-generated magnetic fields and (b) recorded experimenmlly with an $x$-ray pinhole camera from two beams spaced $1 \mathrm{~mm}$ apart on a flat gold target. 


\section{Acceleration of multh-epecles lons in $\mathrm{CO}_{2}$ laser-produced plasmas: Exporiments and theory \\ Fred Begry and Devid W. Forslund

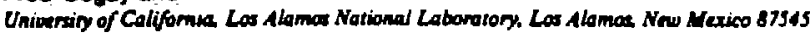

(Received 27 April 1981; sccepted 10 May 1982)

Experimental and theoretical results on the properties of $\mathrm{CO}_{2}$ laser-induced carbun and polyethylene $\left(\mathrm{CH}_{2}\right)$ plasmas at laser intensities of $-10^{15} \mathrm{~W} / \mathrm{cm}^{2}$ are presented. The Thomson parabola technique is used to measure the ion velocity distribution in the underdense expanding plasms which is collisionless and isothe:mal. A model which treats the problem of the collisionless expension of an isothermai c:ectrostatic multi-cold-ion qussineutral plasma will be used to interpret the experimental results. Experiments and theory show that the effect of hydrogen in the $\mathrm{CH}_{2}$ target induces a cutof in the carbon ion velocity distribution. Theory suggests that acceleration field attenuation effects modify the behavior of these plasmas. Data suggest that the space-time evolution of the ion velocity distribution is completed before an ionization-recombination equilibrium is reached. Experimental results from the underdense region are used to estimate plasma parameters near the critical surface, which show that the presence of hydrogen in the target apparently greatly reduces the thermal temperature near the critical surface, probably due to enhanced lateral energy transport.

\section{INTROLUCTION}

In laser-matter unteraction experiments, a basic understanding of the dynamics of the expanding plasma in the lowdensity coronal region is essential to describe the effects of interaction processes, such as laser absorption and scattering, on the implosion efficiency. The inertial confinement process must proceed with high implosion efficiency to achieve successful thermonuclear fission yields. The diversion of absorbed laser energy from the implosion process to the production of energetic ions and electrons and other losses must be minimized.

Much attention has been devoted to the question of ex panding collisionless ton flows in laser-induced plasmas. : In these studies the properties of single-ion flows ware investigated. The expansion into vacuum of a collistonless plasma with two ton species was first treated by Gurevich et al."

In this paper we propose a model which treas the ex. pansion into a vacuum of a collisionless electrostatic plasma with an arbutrary number of ion species. If the intual condituons are properly chosen. the density and velocity flow solu tons in our model are in agreement with the density and velocity flow solutions reported in Ref. 3. The model will be tested with experimental data from laser-induced carbon and polyeth ylene plasmas. In particular, we wish to examine the effect of hydrogen impurities on carbon plasmas. We have fourid that this work is the first to investigate both $\mathrm{ex}$ perimentally and theoretically the :ffect of $h$;drogen impur ities on carbon plasmas.

Results from many investigations ${ }^{4}$ on typical density and temperature profiles of the laser ablatinn process are summarized in Fig. 1 , where four regions have been defined as a function of different states of master.

(1) The undisturbed solid (I) busunded by the shock front (s).

(2) the shock compressed region (II) bounded by the ablarive surface (a),

(3) the overdense heat conduction zone (III) bounded by the critical density layer (c) and

(4) the underdense expanding plasma (IV)

This paper will primarily report on the experimental and theoretical results in region IV, where the underfense expanding plasma is collision-less and isothermal. We attempt to determine the electron temperature in the low-den. sity corona, how that varies with target material, and what are the implications for energy transport.

Many $\mathrm{CO}_{2}$ laser-matter experiments ${ }^{3}$ have been performed at $L x s$ Alamos to investigate the effect of target impurities and surface contamination on the behavior of laserindueed plasmas.

Experimentally, we observe that the pure cirron plas$\mathrm{ma}$ expands isothermally, and that the $\mathrm{C}^{+b}$ ions do acquire asymptotic kinetic energies of approximately $9 \mathrm{MeV}$. The $\mathrm{C}^{+\infty}$ and $\mathrm{C}^{+0}$ ions appear to self-consistently attenuate the

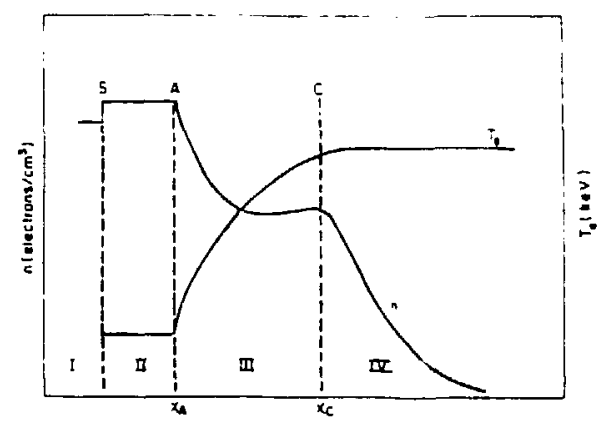

FIG I Typses density-temperature protila versus disunce of the isser ablation process

1075 Pmys. Flude 2519). Septumber $1982 \quad 00319971,82.091675 .1150190 \quad 51982$ Amencan Ingtitute of Phyedes 1675 
Reprinted from APPLIED OPTICS, Vol. 21, page 1886, June 1, 1982

Copyrithe 1982 by the Optical Society of America and reprinted by peimistion of the copyright owner.

\section{Surface plaama abeorption in an Integrating spetsere at high oplical flux lovels}

\section{Richard Kristal}

University of Californis, Los Alamos National Laborato7y, P.O. Box 1663, Los Alamos, New Mexico 87545

Received 2 March 1982.

$0003-6935 / 82 / 111885-03301.00 / 0$

1982 Opticel Jociety of America.

Integrating spheres have been used in the part to make $4 \pi$ scattered light messurements on laser irradiated taryets 1,2 From these measurements absorption of laser light by targets has been inferred. In injestigating the extension of this technique to higher-energy laser fusion measurements, a strong nonlinearity was observed in the sphere response. It takes the form of a saturation of the energy output signal as the input energy is increased. The problem was traced to optical breakdown of the walls of the sphere with significant absorption occurring in the plasma generated on the walls.

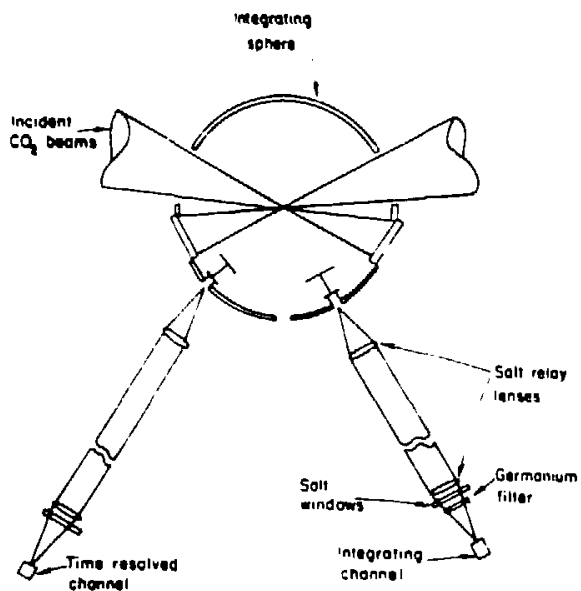

Fig. 1. Integrating sphere experimental configuration
A diagram of the experimental sotup is shown in Fig. 1 . The sphere in $30-\mathrm{cm}$ diem with a diffuse gold-costed interior surface. Two laser beams entes the sphere through $f / 2.4$ entrance holes. The laser source wes the Los Alamos Gemini two-beam $\mathrm{CO}_{2}$ laser facility with a capability of up to $\sim 500$

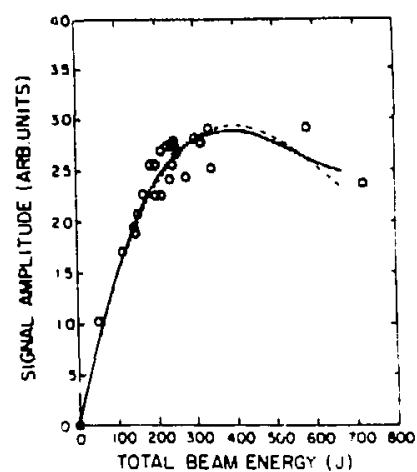

Fig. 2. Energy monitor (integrating channel) response vs lotal beam energy. Solid curve is least-squares third-degree polynomial fit. Dashed curve so computed from time-resolved data normalized to the polynomial fit at $\mathbf{3 0 0} \mathrm{J}$

J/team in a $1-n$ ecc pulse at $\lambda=10.6 \mu \mathrm{m}$. Two detectors were used on the sphere, one integrating and one time-resolved. Both were pyroelectric but of different material and design. The detectors were coupled by salt optics to baffled access holes in the sphere. The spbere and optics were mounted inside the evacuated target chamber $\left(10^{-6}-10^{-5}\right.$ Torr $)$ in which experiments were performed; the optical fetectors were mounted outside the vacuum cinamber.

To use the sphere for target reflectivity measurements it is calibrated in situ by firing the beams directly into the empty sphere. The beams then impinge on solid copper diffuser plates. In this mode and in ideal conditions the signal of 
PHYSICS OF ELECTRONIC AND ATOMIC TOLLISIONS

o Vartb-Hollanu Pubuahung Cumpeny 198:

SURVEY JF ATOMIE PHYSICE ISSLES IH EXPERIMENTAL

IMERTIAL GONF IMENEHT FUSION RESEARC."*

Alian tauer

Iniversity of idifornid

-2511 amos 4at iand idoordtory

E. J. T. Burns

iand a Vational Laboratory

A:omic or icesses impact many gf the interartions important to

inertial confinement fusion. For example. ionization of bound

lectrons represent zne of ine primary stopping mecnanisms for

zotn ions and a iectrons. Atomic processes are 1150 mportant in

predicting the senavior of the very dense plasmas which are ceneral

$: 0$ the success of inertial fusion. Atomic spectroscopy is an

important diaqnostic of ICF plasinas.

INTRODUCTIJH

inertial confinement fusion (ICF) requires the production of high remperature,

gign Jensity slasma. - aser ariven implosions have, for example, been th le to

orgauce plasma densities of the same magnitude as ordinary solids (a few

g/cmi with temperatures of the order of a kilovolt. Atomic processes play

in impor:ant role $n$ the jeneration of such plasma conditions. In dodition,

tiomic spes:roscody nas become in important Jiaynostic of iCF glasma conditions.

itame jrocesses fra giso very imoortant in the onvsics of Iff drivers. Jesign

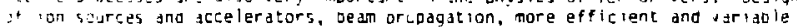

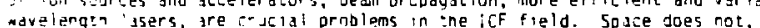

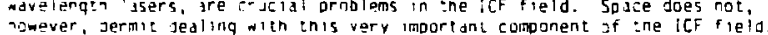

in $=1 \mathrm{~g}$. $i$, we 11 ustrate the generd nature of the intarjations involved in antic and iser driven compression.

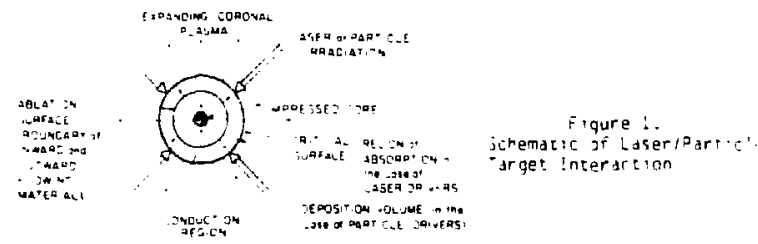

in :he zase of taser heatina, anergy is absorbed at an electron jensity rear the aritical value or the laser involved ! $100^{0} \mathrm{om}^{3}$ for $\mathrm{CO}_{2}, 1021$

$\mathrm{cm}^{-3}$ va:alass? tiomic processes an be aulte important in the ionization und recambination dynamics in the sbsorotion region. 
REPRINTS IN

THEORETICAL PLASMA PHYSICS SINCE

1982 


\title{
Two-Dimensional Simulations of Single-Frequensy and Beat-Wave Laser-Plasma Heating
}

\author{
D. W. Forslund and J. M. Kindel \\ Los Alamos Nalional Laboralory. Los Alamos, New Mexico 87545
}

and

W. B. Mori, C. Joshi, and J. M. Dawson

Universiry of Californa, Los Angeles. Callfornia 90024

(Received 9 Oclober 1984)

\begin{abstract}
Finite-beam, two-dimersiona! particle simulations of single- and double-frequency laser-plasma heatung are presented. In the single-frequency case, Raman backscatter and side scatter initially heat the plasma. Even in the absence of strong forward Rartan scattering, strong subsequent electron heaung is observed. When two collinear laser beams with $\mathbf{J} w \mathbf{-} \omega$, are used, a coherent plasma wave heats the electrons to many megaelectronvolts. In the later case, ion dynamies eventually disrupts the heating process.
\end{abstract}

PACS numbers: $52.65+2,52.25 \sim$ b. 52.50 .Jm

Recently there has been a great deal of interest in underderise plasma heating by intense laser beams. In order to understand the various processes occurring in such a plasma, we have carried out two-dimensional (2D) mobile ion particle simulations using finite laser beams incident onto a large-scale highly underdense plasma. Previous ID simulations have indicated that Raman backscattering (RBS) and forward scattering (RFS) can play an important role in heating the electrons to hizh energies in such plasmas. An important question is whether the heating process is altered by 2D effects. Some of the 2D phenomena are Raman side scattering (RSS), seif-generated magnetic fields, Weibel instabilities, whole beam self-focusing, and filamentation. In this Letter we show that these com. peting effects do indeed occur. Furthermore, at very high laser intensities electrons can be strongly ineated even in the absence of coherent forward Raman scattering.

In order to understand the energies of electrons produced in wave-particle interactions. $2 D$ simulations of beat heating of the plasma by two collinear lasers with frequency difference equal to the plasm 3 frequency have been carried out. In this case a very coherent, large-amplitude plasma wave is set up at wave number $k_{8}-k_{0}-k_{1}$. The time to saturation and the saturation amplitude of the plasma wave electric field is in reasonable agreement with Rosenbluth and Liu's theory. This coherent plasma wave heats the electrons to many megaelectronvolts and produces maximum electron energies predicted by single-particle theory. ${ }^{3}$

The simulations were carried out using the particle code wave on a Cartesian grid in the $x-y$ plane. The plasma is $60 \mathrm{c} / \omega_{0}$ long in both $x$ and $y$. The laser beam has a $\cos ^{2} y$ transverse profile with zero amplitude at $10 \mathrm{c} / \omega$, and $50 \mathrm{c} / \omega_{\text {, }}$ in $y$. Other parameters are $T_{1} / T_{e}=1, \quad \mathrm{~m} / \mathrm{me}=1836$, and $\left(2 \mathrm{kT} / \mathrm{mc}^{2}\right)^{1 / 2}=0.1$. Simulations were carried out with different laser and plasma parameters to cover the parameter regime of interest in both laser fusion and the plasma beat-wave eccelerator. ${ }^{3}$ As representative of these simulations, we discuss two particular cases in thi- paper.

First, a single-frequency laser beam with an $\mathrm{rms}$ intensity $v_{0} / c=0.8$ and a rise time $r$ of $800 / \omega$, is injected from the left-hand boundary in the $x$ direction into a plasma with $\omega_{0} / \omega_{p}=5$. Here $V_{0} / c-e E_{z} / m c \omega_{0}$ is the quiver velocity in the laser field $E_{z}$ and $\omega_{0} / \omega_{p}$ is the ratio of the laser frequency to the plasma frequency. Raman backscatter/stimulated Compton scatter (SCS) is first to appear with a broad frequency spectrum peaked at frequency $\omega_{0}-\omega_{0}$ at time $300 / \omega_{p}$. The RBS/SCS plasma wave with $v_{v} / v_{\text {th }}-1.5$ does trap and hegt a few electrons to a maximum energy of $-100 \mathrm{keV}$. An interesting feature revealed by the $2 \mathrm{D}$ simulations. as shown in the contour plot of Fig. I (a) even al this early stage, is the generation of a magnetic fieid in such an underdense plasma. At this early time, 300 $x \omega_{p}^{-1}$. the magnetic field is typically $\omega_{s} / \omega_{p}-0.1$ ( $-200 \mathrm{kG}$ for $\mathrm{CO}_{2}$ parameters) and has a paitern characteristic of hot-electron filamentation due to the Weibel instability, as shown in the slice plot of Fig. 1(b). On the otber hand, later in time when direct laser heating dominates, the magnetic field can reach $\omega_{c} / \omega^{2},-0.6$ and $i t$ is indicative of a current pattern produced by a single beam of energetic electrons moving down the axis and a relatively colder return current of electrons on the outside of the directed electron beam.

The most striking difference between the ID and 2D simulations, however, is the occurrence of RSS in the latter. RBS evolves very quickly into RSS as the plasma begins to heat up and damping for the shortwavelength plasmons increases. Initially, RSS accurs in the backward hemisphere over a broad range of an. gles but at laier times occurs predominantly in the forward hemisphere. In Fig. I(c) the contour plot of the 


\title{
LA-UR $84-1357$
}

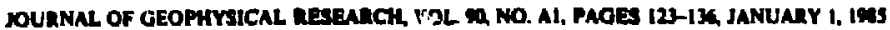

\section{Plasma Heating at Collisionless Shocks Due to the Kinetic Cross-Field Streaming Instability}

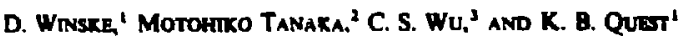

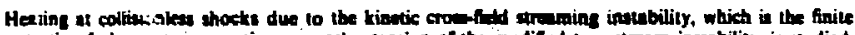

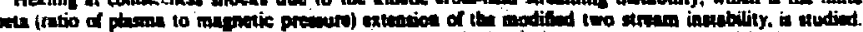

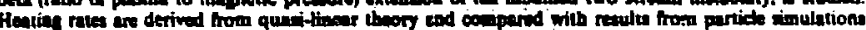

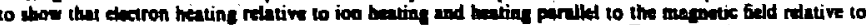

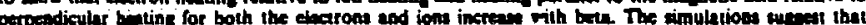

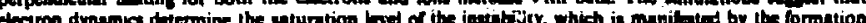

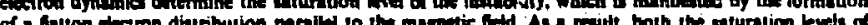

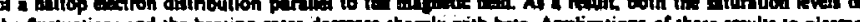

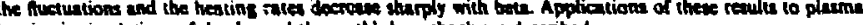
heatine in simulations of teocks and the arnths bow shock are detenbed.
\end{abstract}

\section{INTRODUCTON}

It has been well established for many years that the heating of plasma at collisionless shocks tesults from the interaction with waves generated by mictoinstabilities, through many experimental investigations both in the laboratory and in space of the heating of the plasma and associated wave spectra and theoretical studies of the insubitities and their consequences. Summaries of this work over the past two decades can be found in the reviews by Bistamp [1973], Boyd [1977], David. son and Kral! [1977], Greenstadt and Fredericks [1979], and the collection of invited talks at the 1984 Chnpanan Confer ince on Collisionless Shocks.

The types of waves and the characteristic of the heating which result depend on the sources of the free energy that drive the instabilities, which in turn depend on the nature of the shock. For example, al quasi-perpendicular supercritical shocks the principal dissipation mechanism is the reflection and subsequent thermalization of some of the incident ions. Such reflected ions lead to the observed foot cr pedestal structure in the magnetic field bimodal icn distributions strong ion heating, and long-distance scale over which stoh heating occurs [Montgomery et al.. 1970; Greenstadt et al. 1980]. The reflection process has been clearly observed recently at the bow shock [Paschmann et al., 1982: Sckopke et al., 1983] and in simulations [Leroy et al. 1981, 1982; Forslund et al. 1984]. electron heating for these shocks is usually less intense and occurs over a much shorter distance, typically the length of the magnetic transition starting at the front edge of the foot [Bame et al., 1979]. At quasi-parallel shocks. however, the presence of large-amplitude waves extending over a long cistance abscures the shoct transition and the nature of the heating process [Greenstade et al. 1977]

For quasi-perpendicular shocks when ion reflection is not dominant, the pnncipal source of the instabilities which heat the elections and ions is the cross-field current [e.g. Biskamp. 1973: Lemons and Gary. 1978: $W_{u}$. 1982]. This cross-field current implies a relative electron-ion drift, which gives rise to yanous instabilities. While in some of the laboratory experi-

\footnotetext{
'Los Alamos Nationd Laboratory. New Mexico.

'Institute for Fusion Studics. Hiroshima Untversity, Japan.

Institute for Physieal Science ard Technology. University of Maryland. Colkpe Park.

Copynght 1985 by the American Geophysucal Union.

Paper - imber $\$ A 8112$

$0148-0227 / 85 / 004 A-8112505.00$
}

ments clear idertification of the particular instabilities whych provide the jissipation ean be mude [e-g., Boyd, 1977; Fahr. bach et al. 1981], this has not been true of spacectaft obeerva. tions of collisionless shocks. Such shock encounters usually exhibil a very broad spectrum of electrostatic and electromagnetic noise [c.g Rodriguez and Gurmet, 1975; Gurnutt et al. 1979: Greenstadt et al. 1980]. The identificatien of the individual wave mudes is difteult, hampered by the fact that only Doppler shifted frequencies are mistared and the wave vectors are not resolved. The heiting at such shocks in spave is primarily in the ions [Thomsen et al. this inue], while for shocks in the laboratory the principal heatin often oceurs in the electrons [e.g. Boyd. 1977], although strong ion heating is sometimes obaerved [eg. Danidson and Freddberg, 1976].

The principal instabilities which are thought to occur at shocks are the ion acoustic and "lower hybrid" modes. The ion acoustic instability is a short wavelength (epproximate electron Debye length) high frequency (epproximate ir plasma frequency) electrostatic mode which has been studicd extensively (see reviews by Biskang [1973], Galeev [1976], Bovd [1977]. and Papadopoulas [1977]). It gives rise to a larse resistivity and heats the electron in the bulk of the distribution and the ions in the tail of the distribution, as has been verified by computer simuiation [Dum et al., 1974]. Evidence for the existenx of ion acoustic modes at shocks derives from the obscrved electrostatiz noise and the fact that the width of many skacks is consistent with the threshold condition for the ion acsustic instability [Morse and Greenstade, 1976: Russel et al.. 1982]. The threshold condition has also been used 10 distinguish between resistive and diffusive laminar shocks [.Hellon und Greenstadt, 1984]. The principal dificulty with the ton acoustic instability is that this threshold value. which must be exceeded in order to excite the instability. Is rather large $t_{d} c_{p}>T_{p} T_{r}$, where $c_{d}$ is the relative electron drift speed. $v_{e}$ is the electron thermal speed, and $T_{e}\left(T_{i}\right)$ is the electron (ion) temperature).

In many laboratory experiments as well as ustually at the bow shock. conditions for the excitation of the ion acoustic instability are not satisfied. In such cases the princtpal heating and resistivity is thought to come from the lower hybrid modes. The term lower hybrid is used heie to deseribe various related instabilities: the modified two-stram instability [MCBride et al.. 1972], which is an electrostatic fluidlike mode. the kinetic cross-field streaming instability [Wu er al. 1983]. which is its extension to finite beta (ratio oi plasma to magnetic pressurel and the lower hybrid drift instability [Krall 


\title{
Measurement and Analysis of Near-Classical Thermal Transport in One-Micron Laser-Irradiated Spherical Plasmas
}

\author{
A. Hauer. W. C. Mead. and O. Willi \\ Los Alames National Laboratory, Los Alamos. New Mexico 87545 \\ and \\ J. D. Kilkenny, D. K. Bradley, and 5. D. Tabatabaej \\ Imperial Collere. London. Unied Kingdom \\ and \\ C Hooker \\ Rutherford Appleton Laboralon: Chilton, Didcot. Uniled Kingdom \\ iReceived 10 Autusi 195d)
}

\begin{abstract}
Solid spherical layered targets have been uniformly illuminated at irradiances of $10^{\text {.4 }} 10$ $10^{15} \mathrm{~W} / \mathrm{cm}^{2}$. Extensive diagnostics including time-resolved $x+r a y$ emission and optical prob. ing were used to determine the plasma ablation rate and the plasma blowoff conditions Comparisons with hydrodynamic simulations show that the thermal conduction is well characterized by a nux limit $f_{e}-0.08 \pm 0.02$. with a steep iemperature gradient The $516,$. nass of the heat front was confirmed with time-resolved spectroscopy.
\end{abstract}

PACS numbers. $52.25 . F_{1}, 52.50 \mathrm{Jm}$

Knowledge of energy transport from the region of the laser-light absorption to denset portions of laser-produced plasmas is crucial to an understanding of the laser-irduced ablation process. ${ }^{1-3}$ Exten. sive racent computer simulation work? has led to the expectation that "classical" transport in spherical laser-heated plasmas cen generally be approximated by nux-iimited diffusion with a flux limiter in the range of $f_{e}=0.06-0.2$. Early work, largely involving flat targets. indicaled strong to moderate inhibition of the heat flux relative to classical predictions." Some of the more recent work involving spherical targets has indicated transport levels closer to classical. ${ }^{5}$ with evidence of a penetrating foot in one case." Other work indicates some nux inhibjtion even in spinerical geomeiry.

In the present experiment we have used a comprehensive set of diagnostics to characterize the plasma conditions and thermal transport in a spherically symmetric plasma. We have made timeresolved spectroscopic measurements (using both low- and moderate- $Z$ radiatir.g ions) of the penetration of the heat frons in spherical plasmas. In addition, we have used optical probing to measure simultaneously the density profile of, and the magretic field in, the underdense plasma. Comparisons with hydrodynamic modeling show that (a) all observables are consistent with a high nux limit and (b) the heat front shows no observable foot.

The targets ysed in this study consisted of solid glass spheres $(-160 \mu \mathrm{m}$ diameler), containing $\mathrm{Si}$ and $\mathrm{Ca}$ (among other constituents), coated with three layers: (1) $\mathrm{CH}(0.5$ to $2.5 \mu \mathrm{m}$ thick). (2) Al $(0.1 \mu \mathrm{m})$, and $(3) \mathrm{CH}(0.5$ to $2.5 \mu \mathrm{m})$. The solid glass ball prevented implosion of the target land subsequent inward movement of the critical sur. face).

The targets were uniformly tlluminated with the six-beam, : :06- $\mu \mathrm{m}$, laser facility at the Rutherford Appleton Laboratory. The six beams were focused by $f / l$ lenses with optical axes along the faces of a cube. Incident ir radiance levels were in the range $(1.5-15) \times 10^{14} \mathrm{~W} / \mathrm{cm}^{2}$. The laser pulse had an approximately Gaussian cemporal profile with 0.83-ns full widih at half maximum (FWHM)

The radial intensity proñles of the beams were measured. Small-scale structure results in about $30 \%$ rms variation in intensity. The overlap of the six beams and beam-10-beam energy vriation resulted in about 50\% (peak-to-valley) large-scale variation in incident irradiance across the surface of the sphere."

The primary diagnostic of thermal transport was time-resolved $x$-ray line spectroscopy. $\not X$-ray line emission was observed first from the thin alums. num layer (sensing temperatures of $200-350 \mathrm{eV}$ ) and later from the silicon $(-250-400 \mathrm{eV})$ and calcium $(-500-700 \mathrm{eV})$ in the glass ball. ${ }^{10}$

$X$-ray emission from the target was dispersed (with a thallium acid phihaiate crystal) onto the slit of a streak camera covering 1.7 10 $2.4 \mathrm{eV}$. The spectral and temporal resolutions were about 500 $(\lambda / \Delta A)$ and 50 ps, respectively. A seventh laser beam with very short dutation $(-100 \Gamma . .15$ used 


\title{
Nonlinear evefutlon of the lower-hybrld dritt Inotability
}

\author{
J. U. Brackbill, D. W. Forslund, K. D. Guest, and D. Winskg

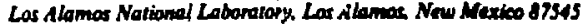

\section{(Received 30 Murch 1984; wocepted 25 June 1954)}

The resules of simulacions of the lower-hybrid drift instability in a neutral sheet configuration are described. The siznulations use an implicit formulation to ralax the uaual time step limilations and thus extend preitious explicit calctiations to weaker gridients, larger mus ntion, and long rimes compared with the linear growth time. The numrerical resulu give the scaling of the aturation: level, heating rale, resistivity, and cross-field diffusion and a demonstration by comparison with - fluid electron model that dissipation in the lower-hybrid drift initability is caused by eisetron kinetic effects.

\section{INTRODUCTION}

The lower-hybrid drift instability remains one of the most widely studied microinstabilities, with many applications to both laboratory and space plasmas. Laboratory devices in which the instability has been predicted by theory to occus, inferied to be present from experimental data or setually observed, include: theta pinches, ${ }^{1-5}$ field-reversed configurations. ${ }^{6-9}$ reversed field $z$ pinches, 10,11 tormac, ${ }^{12}$ undem mirrors, ${ }^{13}$ and bumpy tori. ${ }^{14}$ Regions in space where the instability is thought to exist include both the ionosphere ${ }^{15-18}$ and the magnetosphere lbow shock. ${ }^{19}$ magnetopause. ${ }^{20}$ and magnetotail ${ }^{21,22}$. While all of thesc applications have received some attention, the two configurations most commonly studied in recent years with regard to the instubil. ity involve a neutral sheet geometry: field-reversed configuraticns (FRC's, i.e., reversed field theta pinches) and the Earth's magrietotail. In the first case the lower-hybrid drit instability is thought to be responsible tor the snomalous particle loss across the separatrix, ${ }^{6,7}$ which eventually leads to the destruction of the configuration, and the anomalous flux loss in the region of the field null." The lower-hybrid drift instability has also been invoked as the cause of the enhanced resistivity needed for reconnection in the magnetotail, ${ }^{21.22}$ although the mechanism by which the instability penetrates to the high-beta region at the field null is still unresolved.

The lower-hybrid drift instability is a likely source of turbulence in many of these configurntions beceuse it is rather easy to excits. It is driven by density and temperature gradients (which can be very weak), relatively insensitive to the temperature ratio, and completely suppressed only at very high $(-1)$ beta. ${ }^{23}$ Generally, most of the theoretical work has involved local, linear analysis, including various effects (finite beta, ${ }^{23-27}$ shear, ${ }^{11.24}$ curvature, ${ }^{20.30}$ etc.) as needed for specific applications. Nonlocal analyses have also been carried out, but because of the complerity of the calcu. lations, over much more limited parameter ranges." These calculations have enhanced the credibility of local theory as well as addressed important issues, such as the penetration of the linear eigenmodes to the field null in the reversed field geometry, which is beyond the scope of local theory. Nonlinear theory has also been actively pursued. ${ }^{32-37}$ Efforts have been primarily aimed at investigating the saturation mecha nism(s) for the instability (as will be reviewed later). Besides its intrinsic interest, the saturation level of the fluctuations is neeted when evaluating appropriate quasilinear expressions for the resistivity and heating rates. 3.30

Numerical simulation has also played an important role in verifying linear theory, eliciting saturation mechenisms. and in emphasizing the nonlinear consequences (heating, resistivity, diffusion) of the instability. A number of such calculations have been done in two dimensions ${ }^{40-62}$ or with paeudo-two-dimensional teshriques, ${ }^{3}$ both in simple sheati geometries and in reversed feld configurstions, the, The lat. ter calculations are especially interesting because a characteristically different mode develops at the field aull. presumably as nonlocal, nonlinear consequence of the lower-hybrid drift instability excited outside of this region. Generally, however, the simulations have been limited by the size of present day computers to examining either the evolution of the instubility only through the linear growth and saturation stages or to the use of somewhat artificial parameters (e-g., mass ratios $m_{i} / m_{q} \sim 100$ instead of 1836) to compress time and distance scales. The present study is motivated in part by the development of an implicit particle code venus. ${ }^{45}$ The implicit formulation relaxes the wellknown restrietions on time step and cell size, sllowing the extension of previous calculations to either larger mass ra. tios, weaker gradients, and larger system sizes (allowing more modes to interact) or to long times compared with the linear growth time. The long time behaving is especially important because the instability is usually observed in this state where its sios, nonlinear evolution caures diffusion and heating in laboratory experiments that are srucial when considering the scaling to reactor size devices. Similarly, in space plasmas an understanding of the steady-state microscopic diffusion and heating precesses that occur at the bow shock and magnetopause is needed to determine the overall energy balance in the magnetosphere.

The major portion of this paper is divided into three parts, early tirss, saturation, and late time, corresponding to the aatural phases of evoiution of the instability. In Sec. II (early time) the geometry is descrized, a brief summary of the simulation method is given, and properties of the linear medes obtained from simulation are compared with inear theory. The saturation phase is described in Sec. III. Various saturation mechanisins are reviewed and results of the simuLation are used to show that the scaling of the simulation agrees with several of the theories. The saturation meshanism is further clarified by studying the mode struciure in 


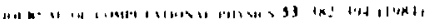

Solution of Elliptic. Fquations Using Fast Pansson Solvers

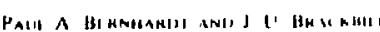

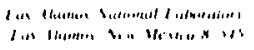

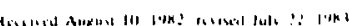

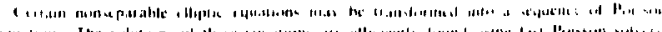

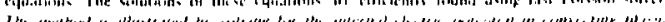

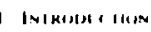

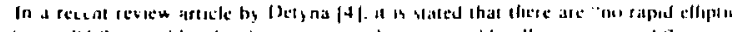

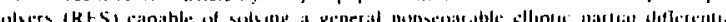

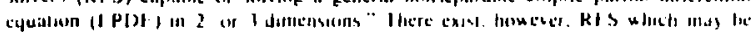

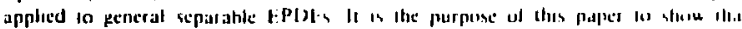

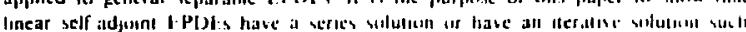

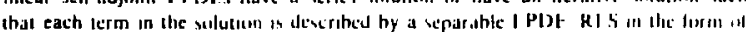

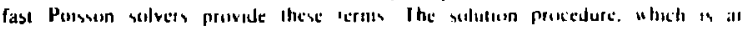

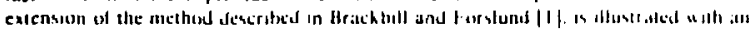

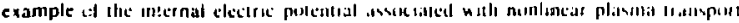

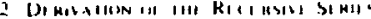

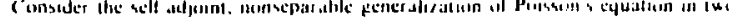
dinenstum

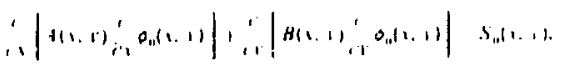

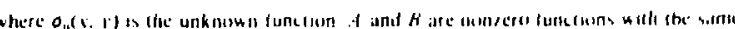

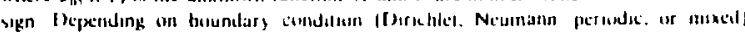

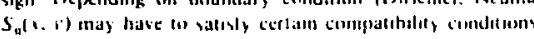

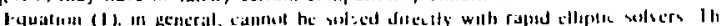
alle

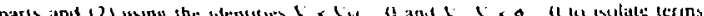

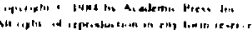

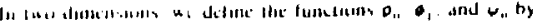

$$
\begin{aligned}
& \text { I. . 1... }
\end{aligned}
$$

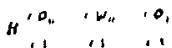

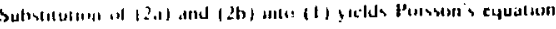

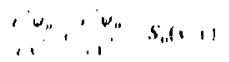

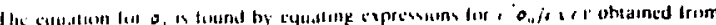
(2a) , and $12 \mathrm{~b}$.

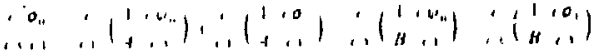

I yuattion (A) in tewtiltion as

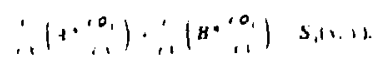

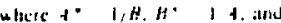

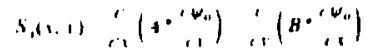

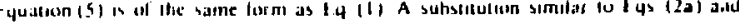
$(2 \mathrm{~b})$ ic

$$
\begin{aligned}
& 4.0,+4,+0 \\
& 0^{10,}, 4,10
\end{aligned}
$$

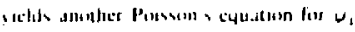

$$
\therefore v_{1}, w_{1} \quad s, \cdots, 11 .
$$

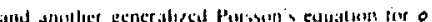

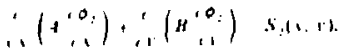




\title{
Ultrahigh gradient particle acceleration by intense laser-driven plasma density waves
}

\author{
C. Joshi", W. B. Mori", T. Katsouleas", J. M. Dawson", \\ J. M. Kindel' \& D. W. Forslund ${ }^{+}$ \\ - Univenity of Califomia La Angeles, Calitomin 90024 , USA \\ + Los Alumos National Laboratory. Ios Aames, New Mexico 87545, USA
}

Space-charge waves driven by resanantly beating iwo laser beams in a high-densiny plasma can praduce ultrahigh electric fields that propagate with velaciries close to $\mathrm{c}$. By phase-locking particles in such a wave, particles may be accelerated to very high energies within a very short distance.

DURING the past four decades, we have witnessed an increase of six orders of magnitude in the output energy of high-energy accelerators, while the cost per MeV has been reduced by a factor of 16 per decade. But can this progress continue? Current accelerators, such as the Stanford linac, have accelerating fields of $200 \mathrm{keV} \mathrm{cm}^{-1}$. However, for particle energies beyond $10 \mathrm{TeV}$, one had to invent schemes that can produce fields or at leasit $10 \mathrm{MeV} \mathrm{cm}^{-1}$. In any particle accelerator scheme, the basic recuirement for obtaining particles with ultrahigh energies is an Intense longitudinal electric ficid that interacts with panicles for a long time. Since highly relativistic particles move nearly at the speed of light $c$, the energy gained by the particles, $\int E \cdot d$, is maximum if the field is made to propagata with the paricles. Extremely !arge electric fields propagating with phase velocities close to $c$ can be produced by space charge waves in a plasma (ionized gas). The maximum electric field that can be produced by such a wave is approximately $\sqrt{n_{e}} V_{c m}{ }^{-1}$, where $n_{\mathrm{e}}$ is the plasma electron density per $\mathrm{cm}^{3}$. Thus for plasma densities in the range $10^{16}-10^{20}$ electrons $\mathrm{cm}^{-3}$, the longitudinal electric ijelds $E_{\mathrm{L}}$, can be as large as $10^{4}-10^{10} \mathrm{~V} \mathrm{~cm}{ }^{-1}$. We now show that such high-gradient, high-phase velocity plasma density waves can be driven by intense laser beams. If paricles could be phase-locked in such waves, this scheme has the potential for accelerating particles to ultrahigh energies in very 5. . on distances.

Theory

If an intense laser beam is propagated in a plasma, then in cenain conditions, the transverse electric field of the laser (which may reach values of $10^{9}-10^{10} \mathrm{~V} \mathrm{~cm}^{-1}$ ) can be very effectively transformed into a longitudinal electric field of a plasma density wave. In the laset accelerator scheme known as the 'Plasma bea wave accelerator proposed by Tajima and Dawson '.7. such a plasma wave can be driven by beating two colinear laser beams, with frequencies and wavenumbers $\left(\omega_{0}, k_{0}\right)$ and $\left(\omega_{1}, k_{1}\right)$, in a plasma resonantly, such that the frequency and the waven umber of the plasma wave are

$$
\begin{aligned}
\omega_{a p w} & =\omega_{0}-\omega_{1} \\
k_{\text {opw }} & =k_{0}-k_{i}
\end{aligned}
$$

To achieve the ultrahigh accelerating gradients, high-density plasmas $\left(10^{16}<n_{0}<10^{26} \mathrm{~cm}^{-3}\right)$ must be used. Also, becassse the laser of frequency $\omega_{0}$ cannot penetrate a plasma whose density exceeds the critical density $n_{e}$ corresponding to the plasm frequency $\omega_{p}=\omega_{0}$, plasmas with densities less than the quarter critical density must be used. Physically, the plasma wave consists of ragions of space charge, which propagate with a phase velocity $v_{p h}$ that is equal to the group velocity of the beat wave $v_{2}=c\left(l-\omega_{0}^{2} / \omega_{0}^{2}\right)^{(1 / 2)}$. These arise because the spatial intensity gradient of the beat eave envelope, which is in the direction of propagation, exerts a periodic force (ponderomotive force) on the piasma at wavenumber $k_{\text {non }}$. The plasma wave is thus an electrostatic wave with $\mathbf{E}_{\text {epe }}$ parallel to kpo.

When two parallel propagating laser beams beat in \& plasma resorantly, the plasma density fluctuations grow rapidly. Using fluid equations it can be shown that for $v_{0} / c \propto 1$, the plasma wave electric field, which is proportional to the perusbed density, initially grows in time with a growsh rate

$$
\delta=\left(\frac{1}{4} \frac{v_{0}(0)}{c} \frac{v_{0}(1)}{c} \omega_{p}\right) s^{-1}
$$

where $\left[u_{0}(0,1)\right] / c=e E_{10,1} / m w_{i g}, c$ is the normalized oscilla sory velocity of an electron in the laser field. Wavebreaking is appronched when the perturbed density becomes as large as the initial density; however, to reach this limit the plasma wave must remgin in phase with the beat wave. As the plasma wave grows, the relativistic effect on the frequency mismatch becomes important ${ }^{2}$ and the plasma wave saturates at a lower amplitude given by

$$
E=\frac{e E_{\mathrm{cpw}}}{m \omega_{\mathrm{ep} w} c}=\left(\frac{16}{3} \frac{v_{0}(0)}{c} \frac{v_{0}(1)}{c}\right)^{1 / 3}
$$

Once the saturation amplitude is reached, the plasma wave amplitude actually begins to decrease, if this process alone acts to sustain the plasma wave ${ }^{3}$.

As the plasma wave is an electrostatic wave, we can use Poisson's equation, $E_{\mathrm{ep*}}=4 \pi e n_{\mathrm{e}} / k_{\mathrm{epw}}$ to estimate the maxinium electric field that we might expect if we assume that the pernurbed electron density is equal to the initial density (the so-called cold
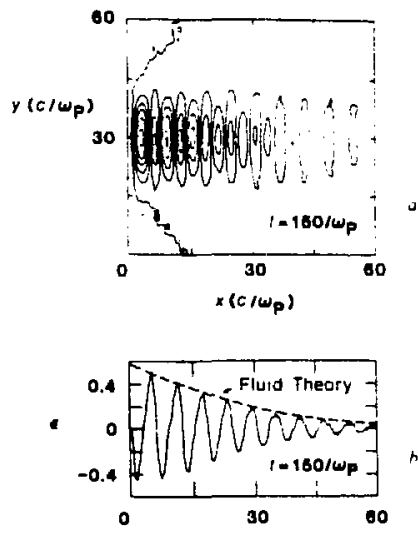

Fle I Potential contours of the space-sharge density wave driven by the two laser beams at lime $150 / \omega_{0}$ (a) section inrough the by the rwo laser bea cenire of the longiudinal electric field of the plasma wave (b). The dotted line in $b$ shows a comparison of the rate of plasma wave build-up as predicted by thi suid theory with that gbtuined in these two-dimensi:ilal simulations. 


\title{
Laser-Induced profile modificatlon: Efiect of electron response
}

\author{
C. H. Altich, R. D. Jonea, and K. Lee

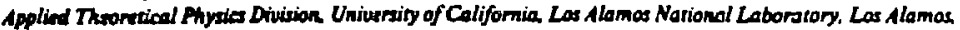 \\ vew Marico 87545
}

(Received 18 November [981; eccepted 8 Mareh 1984)

Püysical processes which occur at the critical surfuce such ss resonance absorption and scattering of the incia ant inser light are very sensitive to the exact form of the laser induced densily profile. The presence of "overdense bumps" in density probles hus been seen experimentally and calcuiated theoretically uing a timple isothermal model. These stuctures are important, in that they could, in an experimenul situation, redect a simiticant amount of light before it reaches the eriticul surfece and considerably afeet the physics occuring within a laser pellet target. A genetalization of a previous calculation by using an atbitrary equation of state (density response of the slectrons to the electrostatic potential $\phi)$ of the form $\rho=\rho(\phi)$ is obtained. In particular, two models for the electronic reaponse (one including the effects of the presence of trapped electrons within the overdense shock structures) calculating the resulting effect on the produced density profiles are investigated. Qualitatively, it is found that the presence of overdense structures and their one-dimensional stability within an underdense plesma is unaffected by the more general electronic response. Conditions for the existence of these structures are discussed and a detailed comparison with full particle in cell (PIC) code simulasions is made, emphasizing the trapped electron model where excellent agriement is obtained.

\section{INTARSOUCTION}

The absurption and cattering of her light at the criti. c) surface o: inser-driven fusion targets is an extremely im. portuat factor in the evencual success of laser fusion. These procesus are very dependent on the exact form of the induced density profiles. Other processes such as energy trans. pors into the plasme, harmonic generation,' megnetically induced surface transport, etc., depend sensitively on the amount of profile steepening at the critical surface. The possible presence of locally overdense structures in the under. dense shelf could dristically afioct the amount of energy arriving at the critical surfece and thus coupled to the plasm. Density modification bes been obverved experimentally, ${ }^{3 \rightarrow}$ and there have been numerous simulations and calculations of it. ${ }^{-17}$ The fact that the laver treepens the density proflle was firn poinied out by Frazlund et al.' The strueture of the profle bas been calculated by Lee et al. ' in the limit of zero aborption and by Stellin werf et a!." "when light is absorted at the critical surfece by the plame. Virmont er al. ${ }^{14}$ and Mulser et al." considered the effects of spherical geometry. Mulser ef al. ${ }^{16}$ ernmined the cave of supersonic to supernonic tranvitiens, but Powers et al. ${ }^{19}$ demonstrated thint these transitions were unstable. The only stable traneitions were subcorie to mpersonic transitions considered earlier. Max and McKee ${ }^{13}$ noted that the laver premure or the preasure from the heated electrons could drive a abock wave into the target.

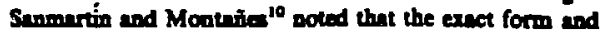
strength of the thock were very dependent on the time dependence of the heatios. Mayer of al. ${ }^{12}$ and Max et al. ${ }^{12} \mathrm{con}$ sidered the effect of phenounenolopically altered heat tow on the dentity proale. And recendly, Jones ef al. "and Forslund

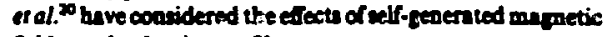
tields on the dentity proille.

- We wish to present in this paper a simple analytic model for the formation of density profiles induced by an incident laver beam and to investigate the effect of the electron re. sponse on the profile steepening and the existence of "overdense burops" or compression-rarefaction shocks. In an earlier paper" we discussed three possible types of density structures for a leser normally incident on an expanding slab of plasme. These structures are pictured diagramatically in Fig. 1. Here the laser is incident from the right with the plasma expanding from the left. The first structure (a) consists of a rarefuction wave connecting the upstream density to a plateau-like area ending in a rarefaction shock at the critical surface. The second structure (b) is a combination of - rarefaction shock at the critical surface and a compression abock downstream. This combination we have denoted as a compression-rarefaction shock. The last structure (c) if basically a piston where all the particles are turned around by the impinging laer beam reflecting them back upstream. This
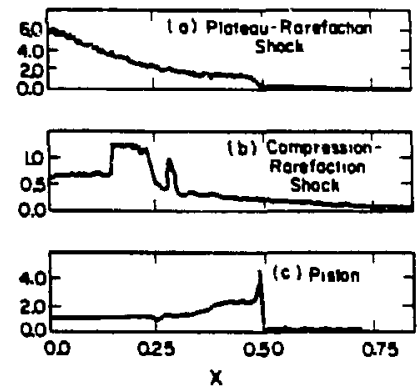

FIO. 1. Three possible density profiles (a) refefaction plateau. (b) compresion-rarefection ahock and ic| piston. 


\title{
Collisionless Dissipation in Quasj-Perpendicular Shocks

\author{
D. W. Forsiund, K. B. Quest, J. U. Brackbill, and K. lee
}

Los Alaunos National Lathurarun

\begin{abstract}
Microscopic dissspation processes in quasi.perpendicular shock are tudied by two-dimensiont olasma simulations in which electrons and ions are trewted as particles moving in self-consustent plasma simulations in which electrons and ions ore treuted is paricles mosing in self-consistent

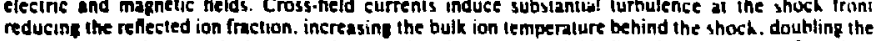

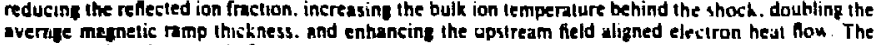
short scale length magnetic fuctuations observed in the bisw shosk ure probabl) associaled wilh this
\end{abstract} turbulence.

\section{INTRODUCTION}

Understanding of the dissipallon of energy in collisionless magnetuc shocks has been sought for many years. Salellite objervations of the earth's bow shock illuminale the strus ture of collisionless shocks including ion reflection 1 Mont gomen et al. 1970: Paschimann ei al. 1982). magnetic field overshool (Heppner el al.. 196\%; Livesey el al., 1982]. thickness of the transition layer (Rissell and Greenstadt 1979). electron and ion heating rates [Bume el al.. 1979]. and high-frequency fields [Greensindt el ol. 1980: Rodrigue: and Gurnelt. 1975. 1976) in the shock layer itself. Compared with these observations. one-dimensional particle simulations [Bistamp and Welter. 1979: Aluer et al. 1971] typically overestimate the number of reflected ions because cross. feld resistivily is absent. One-dimensional hybrid simulations [Lerov ef at . 1981. 1982] that treat the resistive heating phenomenolegically describe the thickness of the foot region and the overshoot region. However. the mechanism for electron heating is unknown and the proper ramp thickness is not obtained in such mnelels. Here. some preliminary results are presented from it $J$-dimensional plasma simulations of quasi-perpendicular shocks that resoive the ion and electron time and space seales. The new results not only reproduce or extend most of the one-dimensional simulation results repored eartier. bu also allow one to identify imporant new electron and ion dissipation processes in the earth's bow shock and to observe upstream field aligned electron heat flow.

\section{Simulation Results}

The bow shock is modeled in two dimensions by a newl developed implicit particle-in-cell simulation technique [Brackbill and Forstund. 1982]. Very high frequency. wo. oscillations are eliminated without removing electron inertia and kinetic effects. In the simulation code VENUS [Bruckbill and Forslund, 1982]. two spatial coordinates and three velocities are treated for each species in the self-consistent. three-component electric and magnetic fields. Numerical dissipation in the code is amaller than the dissipation in the shock due to self-consistent collisionless collective effects.

A magnetic piston is used to drive the shock. The piston. a angential discontinuity which pushes the plasma towards

This paper is not subject to U.S. copyrighe. Published in 1984 by the Ameriean Geophysical Union.

Paper number $3 A 1910$ the left is menerated at the right houndary by is ranoterve electric field $E$, (Figure 1). B! analugy with the earth" dayside magnetopause. the pision is a magnelis harrict through which the magnetic field intensity increases and the number density draps to zero. In the frame mosing with the piston. a super Alfuenic pla'sma hits the barrier. ldunchıng compressional wave, which propagate upsiream and steepen lo form a shock. The angle beiw een upstream $B$ and i in $-k^{-}$ and allous for some moits of the electrons along the tield lines into and out of the shock.

The simulation parameters chosen are for $a, \beta=$ ? plasma with $\beta_{1}=2$ or 0.2 and an Alfven mach number of the shach of 4.5. The units of length in our figures are upatream , w (typically of order $100 \mathrm{~km}$ ). velocits is shoun in units of the upstream Alfvén speed, and time is given in term, of the inverse ion gyrofrequencs, $n_{1}^{-1}$. For economs. We halc chosen $m_{1} / m_{i}=100$ and $\omega_{t+r} / \Omega=20$.

The direction of the ambient field relative to the simulatlion plane can strongly affect the iypes of instabilitie sullow ed in the code. This can be used to advantage in studing shash, For example. in a perpendicular shock propuguung in the direction through an ambient magnetic field in the , diric: tion. the diamagnelic currents are in the : direction and thu uut of the plane of simulation. Thus cruss tiels surren instabilities are not allowed. but pitch angle scaltierine invis. biities are. However, by rotating the amhient field out ot the plane, the cuitents move into the plane allow if crown-ficld instabilities 10 occur. We make use of thin ditferense th illustrate the transition from essentialls zero trometield resistivity to the finite level self-conmistently produced b! cross-field current instabilities.

In Figure I we shou phase space ploth wht $B$ in the simulation $(x-y)$ plane $\left(B_{0}=0\right)$ and $T_{r}: T_{t}=110$. In this plane cross-field curent dnven instabilities cannot he exciled. sn we anticipate a quasi-laminar shuck structure wh substantial ion reflection. The three compunents of telocit! of wn. and electrons are plotted versus the courdinitts a at $! l t=\$$. 5 to show the ion reflection and heating of 1 (In) and electrons behind the shock.

At the time chosen the shock pusiliun is approvmstely $r=12 . / \omega_{p}$ and is separated from the pislon hy a dialance of 6-7.'luy,. As will be seen. the separillion distance corresponds to sevaral ramp thicknesses. sol the processevoperal. ing within the raluplmay the examined separately frum those near the piston. Additionally. The shuck separation iram the left wall is large enough so that buundars efficto mat he neglected. 


\title{
Collisionless Dissipation in Quasi-Perpendicular Shocks
}

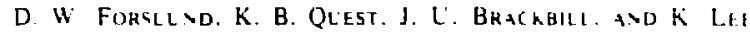

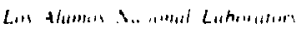

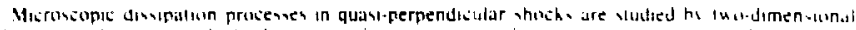

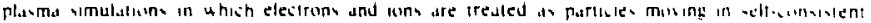

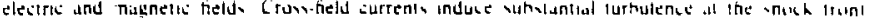

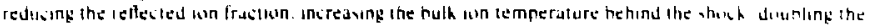

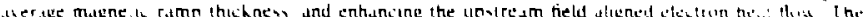

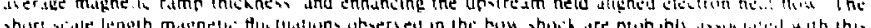
iurtulense
\end{abstract}

\section{ISTRODLTIIS}

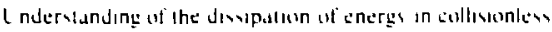
magnews shows has heen sought for mans bists. Saltilit:

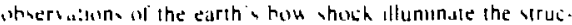

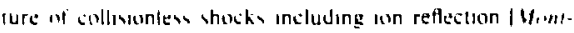

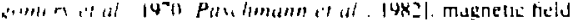

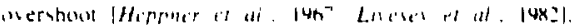

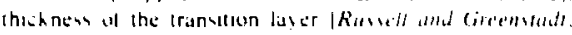

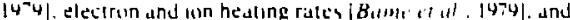

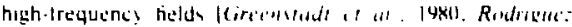

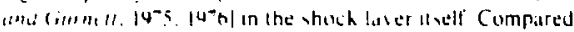
with these athersations, une-Jimenumal particle simulat

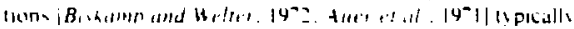
werestimate the number af reflected linn, hecaluse srom

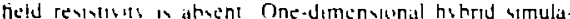

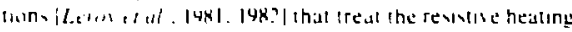

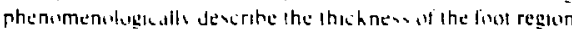
and the olerahoul regen Howeret, the mestiansem for

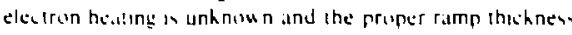
1. nol whianed in alict mudel, Here. vime rieliminars

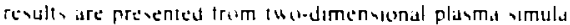

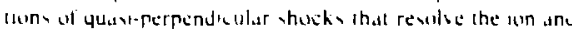
stestron tume and apate heidev I he new resulds now and reproduce or asend most of the one-dimenvonal vimulaturn

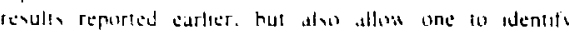

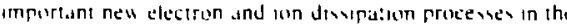
eath h hou shock and to wharse unatream riedd aluened ctele: ron hest Hus

\section{Sutt t+11\%RLSt t Ts}

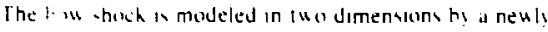
dectoped implist partisle-in-cell amulatisn technicue

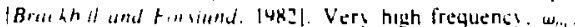

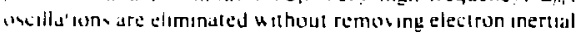
and kinetic etfecls. In the imulation code VEVlS |Brath hill whd forshond. I982l. Ihe vididal colordinates and three velucilse are treated for each species in the self-consmisent. three-component electric and masnells seld Numerical Jismpation in the code in imuller than the dimapation in the

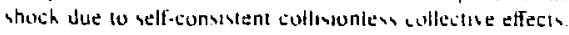

A magnetic piston in used lo drive the hoch The plston. i langential disconisnuty which pushes the plasma cousarda

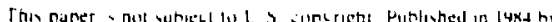
the american Geophrsisul L nom

Puner number $1+1410$

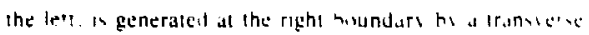

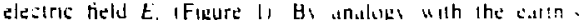
dasvide magnetopause. the pulon on metonctic halfict

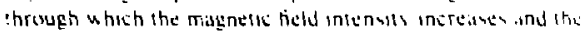
number denvil, Jrops lo kero. In the trume mosing with the puton, a super Alfien/s plusma hul the harrer. launchin! comriresional Hates which propatale upstream and vetepen

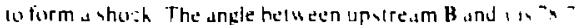
and allou for an: " motion ol the electron along the held lines inlo and out of the shoch.

The imulation parameters shoven are for a $b=2$ mla.m, with $b .=$ ? 1,2 and an Allien mach number ol the vasd of 45 The unue of length in our figureh are upiresm.

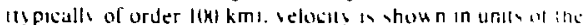
upitream Alfion ipeed. and tame is gixen in terme of the

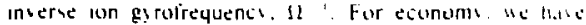
shosen $m, m,=i$ knd $w ., !=21$

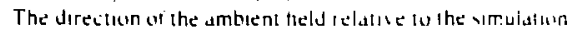

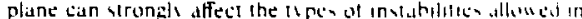

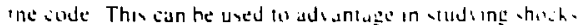
For example in a perpendicular shoth prepolisateng in the ,

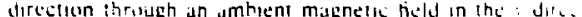

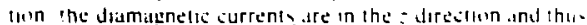

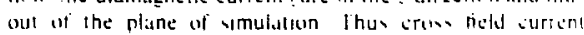

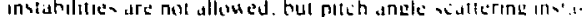

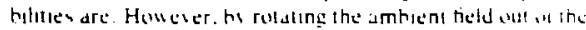
plane. the eurrent more into the plane allowe ine cromeneld

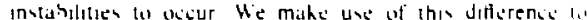

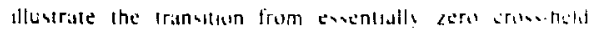

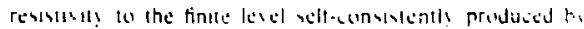
croms-ficid current invidilitis

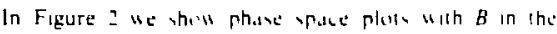

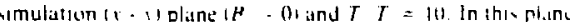

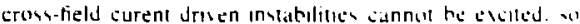
se anlicipatio a quats-laminar shoch vructure with vibutan.

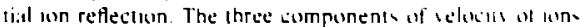

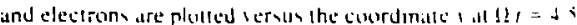

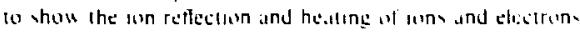
hehind the shock.

Al the time churen the shach pustiun is apprusmallst

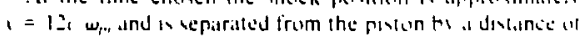

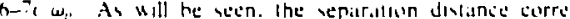
spond to seatral ramp thickneses, whe prosenen uperat ing withon the ramp mals he eximoned veparatels from thase

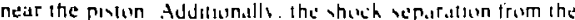
left wall in large enough ab that houndare effers mall he neglecied 


\author{
Magnetic Surface Wayes in Plasmas \\ Foger D. Jonas \\ Applied Theovetical Phye "an Dhision, Los Alamos Natlonal Laboratory, Untiersily of Califomia, \\ Los Alamos. Now Maxico 87545 \\ Reoetrid 16 Jume 1983)
}

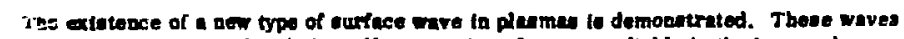

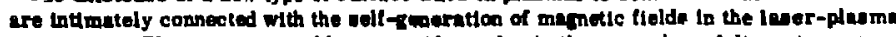
internetion. The wares reamble wichulde modes in that a vumber of dincrete modecen aint. The modea are locellied to within a colliolonlens akls depth of the aurface and.

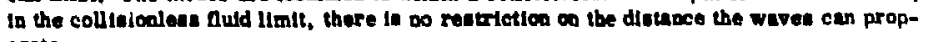
exate.

PACS numbers: $52.35 . \mathrm{Hr}, 52.35 .8 f, 52.40 .5 \mathrm{~d}, \mathbf{5 2 . 4 0 . \mathrm { Kh }}$

Spontaneously generated magnetic flelds in inser-produced planmas have been observed for mang years. ${ }^{1-2}$ These observation, along with their obvious impact on the inertial-confinement luaion program, have been the motivation for the many papers that have appeared on the cubject in the iant decedo."- Transport of energy along aurfaces," anomalouely last plasma blowoff," and ingulation of the lager-heated electron from the trrget interior". (known in the laser fuaion community as (lux limitation) have ell been attributed to propert'es of self-generated magnetic fields. All these phenomenn require sharp discontinuities in plasma properties (e.g., density, temperature, and atomic charge) for their existence. Therefore, the understanding of the normal surface modes in a plasma is crucial to the understanding of these phenomena. In this Letter, I demongtrate the existence of an entirely new set of plasma aurface modes. It will be shown that (1) the self-generated magistic field plays an essential role in the propagation of these waves; (2) a number of discrete modes exist, an in a waveguide; (3) in the collialonless fluid limit, there is no restriction on the ditance the waves can propagate; (4) the waves are localIzed around the surface on acale lengths of the order of a colligionless skin depth; and (5) the phase and group velocities are very dependent on the density and temperature proflles at the surface. While this work has been motivated by programmatic aspecta of the inertial-confinement fusion program, it is felt that the results are quite general and applicable to any plasma that contains sharp density and/or temperature gradlents.

We choose a density profile similar to the one lllustrated in Fig. 1(a). In reglons $A$ and $C$ we require the density gradient scale lengths to be large compared with the scale length of the density jump in region $B$. We will permit the den- aity to vary in the $x$ direction only. The fons are asaumed to be cold and tixed. Quasi charge neutrality is also assumed. Collisiong have been neglected. The temparature prolile is permitted to be arbitrary and no heat flux is permitted. We choove the magnetic field to lie in the $2 \mathrm{di}-$ rection and to vary only as a function of $x, y$, and time. We will look for wavea locallzed in $x$ around region $B$ and propagating in the $y$ direction. The equation for the electron hydrodymam 1ce are

$$
\begin{aligned}
& \nabla \cdot \pi \bar{v}=0, \\
& \frac{\partial}{\partial t}(\vec{\psi}-\bar{\Omega})-\nabla \times \vec{v} \times(\vec{\psi}-\vec{\Omega})=-\nabla \times \frac{1}{m n} \nabla p,
\end{aligned}
$$
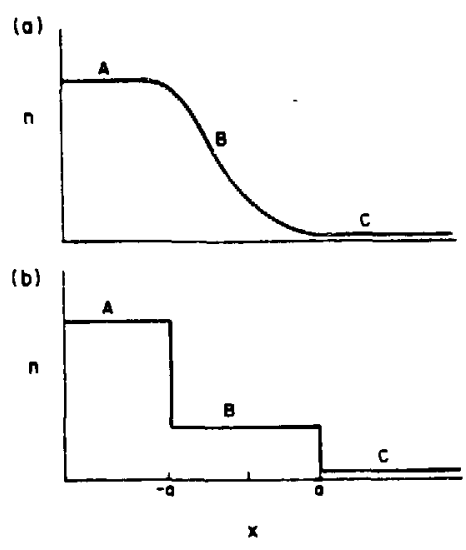

FIG. 1. (a) Density proflie. The surface, region $B$, eepnrates region $A$ from region $C$. (b) Density profile for analytic solution. In region $B$ the probile is nearly nat. 


\section{Implicit Mument PIC-Hybrid Simulation of Coilisional Plasmas*}

\section{RODNey J. Mason}

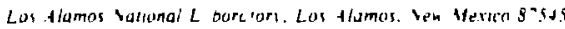

Recerved June 15. 1982, resised January 4. 19\%?

I ielf cunsistent seneme was develuped to model electron transport in evoling plasmas of arhitrary classical collisiunahity The electrons and inns are treated as either muluple Eulerian nutus or collisional particles in cell Parucle suprahermal electrons scater nif ions, and Jrag akainst fuld background thermal elecurons The background elecirons undergo ion inictusn thermal coupling. and bremsitrahlung The con,ponents accelerale in eleciric hields obianed

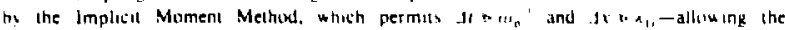
treatment of prohiems $10^{\circ}-10^{\prime}$ tumes mure complex than those accessible with obter explich methods. The flutd description for tint hackpround olasms components permus the mitdeing if transport in tistems spanning more than a $10^{\circ}$ fold change in densits. and eniomnasing soniguous collisional and enllisiontess regions Results are preseried from anolleatlen on the scheme to the mudeling of $\mathrm{CO}$. laser Renerated suprathermal electron ird ipurt in expanding thin foris. and in mult ioul target configuratiuns

\section{NTROOLCTION}

it laser wavelengths exceeding $0.5 \mu \mathrm{m}$ a significant fraction of th: hight abiurthet hy laser fusion targets is deposited in syprathermal electrons. These are marginails collisional and distribute the absorbed energy throughout the target. As thes mose. thes set up self consistent electric fields which dras retury currents in the background. generally strongly collisional. thermal electrons. These fields also set the tons into motion, changing the target geometry. in which the suprathermals must iransport. A detailed model of this complex coupled phenomenologs is required if we arc to develop the intution and understanding needed to engineer the use of suprathermals in the design of targets for high compression Here. our efTerts tou ard the development of such a model were confines to a one dimensional ireatment. However. our choice of approach has been gutued bi the ease with which 11 might be generalized to higher dimensions.

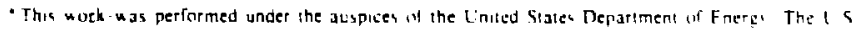

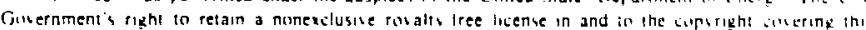
naper. in governmental purposes. is acknum ladged

002199918353.00

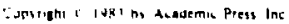

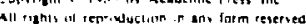




\section{Space-time evolution of the beam-plasma instability}

Michael E. Jones, Don S. Lemons, and Michael A. Mostrom

Aduanced Concepts and Plasma Applications Group.

La. Samas National Labonaton. Las Alamas New Merico 87545

(Received 12 April 1983; accepted 6 July 1983)

Partic!e-in-cell simulations of the benm-plasma instability confirm that the behavior of the interaction can be described as a wave packet that continually grows in both space and time. $A$ consequence is that the energy deposition length of the instability becomes sherier in time, offering increased potential for this interaction to be used as an inertial fusion driver.

The beam-plasma, or two-stream instability, is one of the oldest and most familiar microinstabilities in plasma physics. Recently, there has been renewed interest' in this instability in connection with an inertial fusion driver. In the Anomalous Intense Driver (AID) concept ${ }^{2}$ intense relativistic electron beams, produced by pulsed power machines. would heat a $10^{17}$ to $10^{20} \mathrm{~cm}^{-3}$ density plasma, surrounding an inertial fusion pellet, to multikilovolt electron temperatures. Efficient transfer of energy from the beam to the plasma would be accomplished via the beam-plasma instability. Subsequently, the hot, high-density plasma would provide efficient coupling of the energy to the pellet. By not impinging the beams directly on the pellet, one would avoid the problenes of fuel preheating while retaining the advantage of high energy in relativistic electron beams.

Crucial to the success of this concept is the ability of the beam-plasma instability to deposit the beam energy in a sufficiently short distance. Shor energy deposition distances provide the high-energy densities needed for inertial fusion. The energy deposition length in turn depends on whether the beam-plasma instability is convective/time-independent spatial growth) or absolute (growing temporally at fixed positionl.

Careful and detailed experiments on the beam-plasma instability have shown that a time-independent spatial growth of the instability is obtained. ${ }^{t-6}$ For this reason the inste jility is generally believed to be convective. Criteria for when an instability is convective nr absolute has been given by Briggs.' However, he noted that for the simple case of the cold-fluid one-dimensional, electrostatic dispersion relation for the beam-plasma system, his "pinch-point" analysis fails to give a definitive answer about the nature of $t$. instability.

The dispersion relation for this case is well known:

$$
1-\omega_{p}^{*} / \omega^{2}-\omega_{b}^{2} /\left(\omega-\left.k v_{b}\right|^{2}=0\right. \text {, }
$$

where $\omega$ is the Laplace transform (in time, $t$ ) variable, $k$ is the Fourier transform (in space, $z$ ) variable, $v_{b}$ is the beam veloc tty. and $\omega_{p}$ is the electron plasma frequency. To generalize this dispersion relation to relativistic beams. one simply replaces the square of the beam-plasma frequency $\omega_{b}^{2}$ by $\omega_{b}^{2} / \gamma^{\prime}$, where $\gamma$ is the Loreniz factor of the heam. Such dispersion relations are obtained by taking the Laplace-Founer transforms of a set of equations describing the system. They are often analyzed by assuming $k$ to be real and known and solving for complez $\omega$. The resulting solutions describe the temporal growth of spatially periodic disturbances. Conversely, one may solve for real $\omega$ and complex $k$ to obtain spatally growing solutions. However, the actual response of the system must be obtained by inverting the Laplace-Fourier transforms, taking into accourt the sources of the disturbances. The inverse transforms for the system with the dispersion relation given by Eq. (1) for weak beams can be approximated using the saddle point method., $A$ delta function source at $t=0$ and $z=0$ is assumed, thus obtaining the Green's function response of the system. The result is

$$
E \sim\left\{\begin{array}{l}
\exp \left\{i \omega_{\rho} r \mid \exp \left[(3 v 3 / 4)\left(\omega_{b} z / v_{b}\right)^{2 / 3}\left(\omega_{\rho} \tau\right)^{1 / 3}\right],\right. \\
v_{b} t>z>0, \\
0, z<0, \varepsilon>v_{b} t .
\end{array}\right.
$$

where $E$ is the electris field and $\rightleftharpoons=f-z / v_{0}$

The respoisse given in Eq. (2) can be called "absolute" since $E$ grows in time fo: a fixed position 2 . However, for fixed $t$ there is also growth in space, reminiscent of a convective instability. In fact, Eq. (2) describes a wave packet that moves in space and grows as it moves. It is easy to show that the peak of the wave packet moves at a velocity $2 / 3 v_{b}$, which is the group velocity of the most unstable wave obtained from the usual dispersion relation analysis for real $k$ and complex $\omega$. Furthermore, in a reference frame moving with this velocity, the electric field grows at a maximum growth rate obtained from the dispersion relation analysis.

Although the wave-packet analysis is for an extremely simple model of the beam-plasma system. inclusion of other physical effects, such as finite plasma and beam temperature, does not alter qualitatively the wave-packet behavior. ${ }^{10}$ To further investigate this behavior and its relevance to iner tial fusion drivers, several particle-in-cell simulations have been performed. Most previous simulations of the beamplasma instability have used periodic boundory conditions. This forces $k$ to be real and the linear growth rate for this case is observed. However, the space-time behavior described by Eq. (2) cannot be observed unless aperiodic boundaries are used, as is the case for the simulations presented here. Figure 1 illustrates the growing wave-packet behavior observed in one of the simulations. The computer code used here is CCUBE, a 2!-dimensional, relativistic, electromag. netic PIC code." An annular relativistic $(\gamma=2)$ electron beam with inner radius $r,=10 \mathrm{c} / \omega_{p}$ and outer radius $r_{0}=20 \mathrm{c} / \omega_{p}$ is injected into a plasma-filled conducting cylinder with a radius ${ }_{c}=30 \mathrm{c} / \omega_{p}$ and length $L=100 \mathrm{c} / \omega_{p}$, The beam density $n_{b}$ is $1 \%$ of the plasma density $n_{p}$. There is also an axial magnetic field imposed that gives an electron cyclotron frequency $\omega_{1}=01 \omega_{p}$. The cell size is $0.5 \mathrm{c} / \omega_{p}$ on each side and about $10^{\circ}$ particles are used. The lime step is $0.2 \omega_{z}$ '. The graphs in Fig. 1 show the axial electnc field located at the radial center of the beam as a function of axial 


\title{
Retura-Current Heating and Implosion of Cylindrical $\mathrm{CO}_{2}$-Laser-Driven Targets
}

\author{
A. Hauer and R. J. Mason \\ Los Alamos National Leboralory. Lniversity of Califomia. Los Alamos, New Mextco 875t5 \\ (Recelyed 1 Aprll 1983)
}

The Helios laser system has been used to deliver $2.3 \mathrm{~kJ}$ to the capped end of $0.75-\mathrm{mm}$ long, $130-\mu \mathrm{m}$-diam hollow rods of $5-\mu \mathrm{m}$ wall thicknese. Soft-x-ray plnbole pictures demonstrate the cyllndrical Implosion of thene targets. The moasured 130-eV core temperatures from the Nitered plctures and the $7 \times 10^{6}-\mathrm{cm} / \mathrm{s}$ collape volocity from optlcal gireak pbotcgraphs are consistent with beation by a $0.8 \times 10^{4}-\mathrm{A}$ return curreat, representing the recycling of $15 \%$ of the bot-electron emisiton.

PACS numivers: 52.50.Jm, 52.25. F1, 52.30.+ r, 52.70.-m

At intensities which exceed $10^{10} \mathrm{~W} / \mathrm{cm}^{2}$ most of the energy absorbed from $\mathrm{CO}_{2}$ lagers goes dIrectly into a relatively small number of highly energetlc ( $>200 \mathrm{keV}$ ) suprathermal electrons. ${ }^{1}$ This energy must be tranglerred to a mucin larger number of localized thermal electrons to ac compllsh lager fusion. Since direct classical coupling of the suprathermals to the background plasma has proven inelficient, we have initiated an elfort to use the solf-generated flelds in specialized targets to improve the energy transfer. Charge imbalance develops as guprathermals leave a lager spot. The resultant $E$ flelds can draw a return current, If an appropriate path is provided. Benjamin et al. ${ }^{2}$ haye demonstrated the existence of these currents, and their ability to heat a target support stalk. This Letter re ports the first experimental results from targets designed to use the return currents to heat and implode a thin-walled hollow cylinaer $\rightarrow$ to produce a micro $Z$ ptich.

A schematic of the "augmented return current" (ARC) targets used in the experiments is given in Fig. 1. Four tightly focused $\mathrm{CO}_{2}$-laser beams from the Los Alar: a Hellos laser system im pinge on the capped and a a long hollow, low $-z$ cylinder, coated with a thin layer of metal (gold or alumimum). The cylinder is mounted at the center of a large high- $Z$ disk. Suprathermals generated at the focus will drift toward the disk in the plasma surrounding the cyiinder. A thermal return current is drawn back along its walls. Resistivity of the walls resuits in Joule heating of the thermals. The $B$ field from the resultant current loop can implode and further heat the cylinder.

Experiments were performed on targets with a variety of diameters and lengths. The smallest cylinderg were matched to the minimal laser spot diameter. Their length was get at roughly 5 diameters to maximize the aspect ratio for cur- rent-related effects, undr $r$ the constraint of minimum mass- or muximum temperature gan trom energy deposition. The minimum wall thickness was set by limits on structural integrity. The metal layer was added to provide a brighter signature in $x$-ray pinhole pictures. The earlier experiments ${ }^{2}$ recorded currents from electrons escaping to the walls of the target chamber. The ARC targets were designed to augment thls current through the axial alignment of the cylinder and beams, with a cylinder much thicker than the earlier $10-\mu \mathrm{m}$ stalks, and with the addition of the collector disk for possible suprathermal entrainment. The presence of thermoelectric, $\nabla n \times \nabla T B$ fields has been recognized for some time. ${ }^{-\rightarrow}$ The cylindrical return currents and amy pinch effect should serve to enhance thege fields. Suprathermal drift down the cylinder is consistent with a $B$ tield, directed es in Fig. 1, and with an outwardly tiarected $E$ fleld for contalnment of the electron cloud. The axial $E$-tleld component, drawing the return current againgt resistivity, introduces a "tilt" in the $E \times B$ drift, which should aid capture of the suprathermals by the cylinder wall. Alternative $l y$, the $B$ field around the cylinder should help to shieid its interior from suprathermal preheat." The return-current effects discussed here will be reduced to the extent that deposited energy is lost to fast-ion blowoll. However, cylindrical geometry may serve to reduce fast-ion losses, as compared to those from foils, since the area

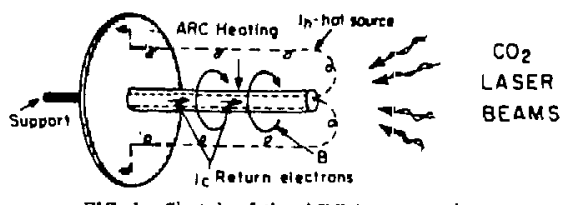
FIG. 1. Sketch of the ARC tark:" . "ikn 


\title{
Eigenfunction analysis of the beam-plasma instablity with finite radlal dimensions
}

\author{
Michael E. Jones
}

Applied Theorerical Physies Division. Las Alamos National Labonatory. Los Alanios. New Mexico 87545

(Received 12 October 1982; accepted 16 March 1983)

A linear eigenfunction analysis of the beam-plasma instability for an annular beam interacting with a plasma filling the space between the conductors of a coaxial transmission line is presented. The beam and plasma electrons are modeled by relativistic cold fluids and the dispersion relation is three-dimensional and fully electromagnetic. Particular attention is given to the radial eigenfunction structure and extensive numerical examples are presented. The behavior of the dispersion relation with various parameters is interpreted in terms of the infinite homogeneous theory. Simple analytical approximations for the dispersion relation for annular beams are given. Also, particle-in-cell simulation results are compared with the predictions of the linear analysis

\section{INTAGOUCTION}

It has been proposed that intense annular relativistic electron beams be used to efficiently heat plasmas in the density range of $10^{17}-10^{20} \mathrm{~cm}^{-3}$. The resulting hot annular plasma has a variety of applications including utility as an inertial confinement fusion driver.' Recent experiments designed to test the feasibility of heating annular regions of dense plasma hive used beams with a radius of $1 \mathrm{~cm}$ and a thickness of $0.2-0.3 \mathrm{~mm}$ at an energy of $3 \mathrm{MV}$ (Ref. 2). For these very thin beams it might be expected that the usual assumptions of an infintte homogeneous beam-plasma system are inapplicable. The purpose of the present work is to analyze the effect of finite radial dimensions on the linear stability of this beam-plasma system.

This work represents a get:eralization of previous anal. yses with finite radial dimensiuns to the case of an annular relativistic beam interacting with a plasma which fills the annular region between the conductors of a coaxial transmisston line as illustrated in Fig. 1. The dispersion retation preented here is three-dimensional, fully electromagneuc, and is obtained by assuming a relativistic cold-fluid approximation. Although the emphasis is on annular beams, the dispersion relation for solid beams is contained as a subset of the more general analysis. Extensive numerical results are presented. Particular emphasis is given to the relationship of the dispersion relation for an annular beam to that for the infinite homogereous sycrem.

The tream-plasma instability is one of the most thoroughly studied microinstabilities in plasma physics. For a summary of the analyses of nonrelativistic beams, including the effects of finite radial dimensions, the reader is referred to the review article by Briggs.' A review of the work with finite radial dimensions for relativistic beams and electromagnetic waves is given by Aronov, Bogdankevich, and Rukhadze." Perhaps the carliest analysis of a finite relattvistic beam was by Frieman et al. ${ }^{5}$ who studied the case of a solid beam in an infinite plasma. Recent refinements have included the effects of finite external magnetic fields and beam iemperature.

The radial eigenfuncuons are examined in detail in the present analysis. It is found that the unstable waves are evarescent outside the beam annulus. This behavior results in an effective quantization of the wave vector perpendicular to the beam direction, $k_{1}$. The quantization is determined by the beam thickness. The stable waves are found to be radially oscillatory everywhere and possess an effeciive $k_{1}$ quantization deiermined by the dimensions of the conductars. Annular beams are found to possess two unstable surface waves. These waves always have lower growth rates than the buik modes. One of these surfaces modes is found to be analogous to the $k_{1}=0$ wave of the infinite homogeneous theory.

To a good approximation it is found that the unstable waves for thin annular beams correspond to the waves in the infinite homoge:szous theory with $k_{1}$ replaced by $k_{t}=\mid k$, $+(\mid) \pi / \tau$, where $\tau$ is the beam thickness and $k$, is an integer. and as mentioned earlier, there is a mode for $m_{1}=0$. The growth rate of the most unstable mode is given ty Eq. $(14)$.

Finally, the usefulness of the eigenfunction analysis is tested via particle-in-cell simulati:: The results indicate that while the eigenfunctions probably do not describe the entire response of the system, many of the features of the present analysis are observed in the sinulations.

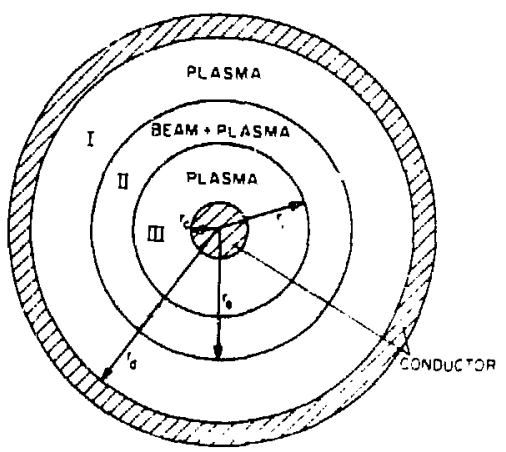

FIG 1 Most general gecmetrical cenfiguration considered in the anais sts 


\section{Shear-driven Inatabllitles of annular relativistlc electron beams in vacuum \\ Micheel A. Mostrom and Michael E. Jones \\ Intense Panicle Beam Theory Group. Los Alamos National Laboratory. Los dlamisi New Mfexico 8754}

(Received 2 February 1982; accepted 7 February 1983)

The study of instabilities driven by azimuthal shear (diocotron) and axial shear is extended to annular relativistic beams. The analysis is done nonrelativistically in the beam fra! : $:$ 'there the instability is assumed to be electrostatic. and the dispersien relation is then transformed back to the laboratory frame where magnetic perturbations are non-negigible. This requires keepirg finite axial wavenumber $k_{z}$ in the analysis. The axiai and azimuthal shears are related through the self-consistent equilibrium, assuming emission fron an equipotential cathode. Asial shear destabilize the diocotron modes at higher azimuthal wavenumbers $l$. It alsn prodl: :es a set of modes including $l=1$ modes, thet are unstable as the result of wave-particle intera:-ions. The annular configuration introduces an important second pole in the differential equaticn.

\section{INTRODUCTION}

The diocotron and $v_{z}$-shear-driven instabilities in nonrelativistic annular electron beams are generally considered to be well understood, but there are some important omis. sions. Levy's work on the diocotron instability ineluded significant cylindrical effects' but ignored the radial shear in the axial velocity $v_{z}$ and considered only the case $k=0$ li.e. no $z$ vanation) which correspond to the maximum growth rate. Rome and Bnggs included $k$, shear with all $k$ i with finite $b_{z}$ shear, the growth rate is not maxinized at $k=0$ ) but considered only a cylindrical solid beam which eliminates the diocotron instability. ${ }^{2}$ Antonsen and $\mathrm{Ott}$ investigated simultaneously the diocotron and $u_{z}$-shear instabilities for al $k$ but limited themselves to planar slab beams. ${ }^{j}$ In an earlier paper Jones and Mostrom studied the diocotron insiability for all $k$ in annular beams but treated $u_{z}$ shear only to the extent of obtaining conditions for its neglect. ${ }^{4}$ The first step in the present paper is to generalize the nonrelativistic theory by including both the diocotron and $u_{z}$-shear instablities for all $k$ in annular beams.

The main application of these theories has been microwave generation in crossed feld magnetrons. In recent areas of experimental and theoretical interest. intense annular relativisuc electron beams are employed. These include free eleciron lasers." multigapped accelerators." and the anomalous intense driver fusion concept.' Because the nonlinear state of the diocotron and $v_{z}$-shear instabilities can disrupt the beam by causing filamentation, ${ }^{8,0}$ it is important to understand these instabilities in the relativistic regime including cylindrical effects.

There have been several recent attempts at such a relativistic cylindrical theory, but again all suffer from various degrees of omission of important effects. Kapetanakos. Hammer, Striffer, and Davidson found an $\omega=0$ spatial growth in an annular beam. "But. they incorrectly assimed that the instability is electrostatic in the lab frame and also ignored $r$ : shear. Uhm and Siambis" and also Chen and Palmadesso" " investigated the diocot ron instability in annular beams but limited themselves to $k=0$ in the lab frame and ignored $t$; shear Both $t^{\prime}$; shear and relativisitc effects shiff the maximum growth rate away from $k=0$. In the previously mentioned paper, Jones and Mostrom oblained the diocotron dispersion relation for all $k$ in the lab frame by a transformation from the beam frame, but found that neglert of $u_{z}$ shear limited their results to small azimuthal mode number / (Ref. 4). In none of the above relativistic theories was the $v_{2}$-shear-driven instability studied which can destabilize the $I=1$ mode. In the present analysis we generalize the previous relativistic cylindrical analysis of Jones and Mostrom by including $v_{2}$-shear effects. It can also be thought of as a relativistic cylindrical generalization of the nonrelativistic planar finite $U_{z}$-shear analysis by Antonsen and Ott.

The dispersion relation analytically obtained here is studied numerically and compared with previous analysis where appropriate. For the diocotron instability, the primary effect of finite $v_{x}$ shear on the growth rate is to maintain and enhance the instability for much larger $/$ than otherwise would be possible. This is accomplished through frequency shifts of the component waves in the rosonant wave-wave interaction. For the $v_{z}$-shear instability, an annular besm introduces into the differential equation for the perturbed electrostatic potential an important second pole that is not present for a constant density cylindrical solid ${ }^{2}$ or planar slab $^{3}$ beam. The present analysis treats this pole to only second order but serves to identify this problem and ascertain its imporance. For large $l$, this second pole becomes importan: even for the diocotron instability.

\section{EQUILIBAIUM KODEL}

The electron beam is assumed to be initially in an azimuthally symmetric equilibrium inside a conducting dnft tube of radius $d$ as illustrated in Fig. 1. A large externally applied axial magnetic field is required for equilibrium. The azimuthal equilibrium velocity $v_{\theta 0}|r|$ of the elecirons is an $E \times M$ rotational drift induced by the applied magnetic field $B_{z}$ and the radial electric self-field $E_{*}$ ( $\left.r\right)$ of the unneutralized beam. Space charge effects radially shear the electron axial velocity $i_{s 0}(r)$. The beam frame reierred to throughout this paper is defined such that $k_{r o}=0$ at $r=a$, the inner beam radius. Using the radial force balance equation, conservation

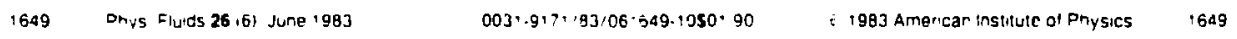




\title{
Field saturation for arbltrary temperature in resonance absorption
}

\author{
8. Bezzerides and S. J. Gitorrer

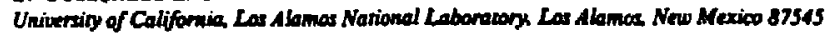

(Received 1 April 1982; accepted 28 December 1982)

A nonlinear thesry of a driven, inhomogeneous, one-dimensional plasma of arbitrary temperature is presented. The model is solvert numerically and analytically and detailed expressions are given for the enturated electric feld excited at critical density. The results of the calculation are valid for arbitrary tempenture and connect in a continuous manner the limits of cold wavebreiking and linear convective saturation. Comparison of the results of the calculation with. partisle simulations is made and good agreement is cblained.

\section{INTRODUSTION}

Since the clessic work of Ginzberg, 'the study of resonance absorption in a warm pixsma has advanced by the use of particle sisnulation techniques. ${ }^{2-4}$ Whereas the Ginzberg work achieves a saturated electric field level solely through the convective stabilizntion of the field due to finite buckground temperature the simulations show the importance of hot-zlectron production for stabilization at sufficiently high values of the external field. Thus for strong driving, one may anticipate the diminished importance of the background temperature for establishing the final electric field level. Wavebreaking in cold-piasma models ${ }^{3}$ has pruvided useful scaling laws when the background temperature can be completely -ignored. Although the precise connection between wavebreaking in a fluid model and hot-electron production is not fully understood, it is argued that the onset of wavebreaking in the fiuid theory signals the creation of hot electrons. The time of onset of wnvebreaking is then used to estimate the saturated field level of the excited electric field.

To assess the relative importance of background temperature upon the level of the saturated electric field in resonance absorption, Kruer combined results from the cold wavebreaking model ${ }^{3}$ and a nomozeneous undriven waterbag model. This calculation provided a prediction of the effect of plasma temperature on wavebreaking amplitudes. However, the results are essentially limited to relatively low temperatures, since in some sense the approach used relies on a perturbation in temperature about the cold-plasma case. One may also question the consistency of Kruer's calculation; however. the predictions are in reasonable agreement with the results of particle simulations at least for relatively low temperatures.

In this paper we present a consistent thuid model of a driven, inhomogeneous, warm plasma. We show how one may obiain the saturated value of the electric field excited at critical density for arbitrary temperature. Of course, in such a model, the effect of hot-electron production is not included. Nevertheless, here we find, through comparison with particle simulations, that accurate saturated field levels are obtained from numerical solution of the fluid model and by an approximate analytic theory. In Sec. II, we outline the model, providing an approximate solution in Sec. III, and we present tite numerical results and comperisons in Sec. IV, ending with some concluding comments.

\section{LAOAANG!AN FLUID MODEL}

Consider a one-dimensional plasma of mobile electrons and fixed ions. The equations of contunuity and momentum along with Poisson's equation provide a complete fluid description, given the pressure $P$.

$$
\begin{aligned}
& \frac{\partial n}{\partial t}+\frac{\partial}{\partial x}(n v)=0, \\
& \frac{\partial v}{\partial t}+v \frac{\partial v}{\partial x}=-\frac{e}{m_{e}}\left(E+E_{0}\right)-\frac{1}{m_{e} n} \frac{\partial P}{\partial x}, \\
& \frac{\partial E}{\partial x}=4 \pi\left[n_{0}(x)-n(x, t)\right] .
\end{aligned}
$$

where $n$ and $u$ are the fluid density and velocity, $e$ and $m_{r}$ are the electron charge and mass, $E$ and $E_{0}$ are the self-consistent and driving electric fields, respectively, and $n_{0}$ is the fixed ion density. If the gradient of the heat flux is assumed to be zero, then it is a simple matter to truncate the fiuid equations to obtain the pressure in terms of the density. Such an assumption ignores the presence of hot-electron production. a condition consistent with the early stage of time evolution of the plasma fiuid prior to the inset of wavebreaking. We find 3.4

$$
P / n^{3}=\text { const. }
$$

For this one-dimensional set of equations it is convenient to introduce the Lagrangian transformation $x=x_{0}+\delta\left(x_{0}, \tau\right)$ and $t=\tau$, where $\delta\left(x_{0}, \tau\right)=\int \overline{0}\left(x_{0}, \tau^{\prime}\right) d \tau^{\prime}$. with $\dot{u}\left(x_{0}, \tau\right)=v(x, t)$. Using the above transformation we obtain the following equation of motion for $\delta$ :

$$
\begin{aligned}
\frac{\partial^{2} \delta}{\partial \tau^{2}} & +v \frac{\partial \delta}{\partial \tau}+\int_{x_{w}}^{x_{n}+\delta} \omega_{p}^{2}\left(x_{0}^{\prime}\right) d x_{0}^{\prime} \\
& =-\frac{e E_{D}}{m_{e}} \sin \omega \tau-\frac{1}{m_{e} n_{0}} \frac{\partial \tilde{P}}{\partial x_{0}} .
\end{aligned}
$$

where $v$ includes dissipation due to linearized Landau damping and a spatially dependent ad hoc damping introduced to avoid boundary anomalies in the numerical solution. The third term in Eq. (2) accounts for the plasma-restoring force for the general ion density profile; however, in what foilows 


\title{
Magnetohydrodynamics in Laser Fusior: Fluid Modeling of Energy Transport in Laser Fargets
}

\author{
J. U. BRACKBILL AND S. R. GOLDM.AN \\ Los Alamos Nalional Laborators
}

\begin{abstract}
Flud models for two-dimensional. electron energy transpon are examuned it is shown that enhanced lateral transport due to parucle dnifs in self-generated magneuc jelds occurs when cerrain transpont terms are flux-limited by artuficiatly enhancing collisions. With flux limiting. fluid models reproduce the essential le atures of numerical simulations of transport in $\mathrm{CO}_{2}$ laser target experiments
\end{abstract}

\section{Introduction}

New results (cf. [1] [3]) suggest that magnetic fields play a central role in energy transport in laser target experiments. In recent numerical simulations of collisionless plasmas (cf. [1]) using the VENUS code (cf. [4]) and laser experi ments at 10.6- $\mathrm{mm}$ wavelength ( $\mathrm{cf}$. [3]), it is observed that lateral energy transport is convective rather than diffusive. The convection is associated with the formation of a magnetized sheath on the target surface.

Although magnelic fields have been observed before icf. [5], [6]) and modeled theoretically (cf. [7], [8], [10]). their role in electron energy transport was though to be a passive one. Simply by their presence at the periphery of a laser spot. they were believed to block electron energy transport across the target surface cf. [9]:.

The new transport model is different from the old in two respects. First. a thermal magnetic wave icf. [10]) rapidly propagates the magnetic field away from the source across the target surface. Thus, the magnetic fieid covers the target surface instead of being localized to the periphery of the laser spot as previously believed. Second, the hot electrons generated by the absorption of laser energy drift in the magnetic field and convect energy away from the source. As a result, hot electrons uniformly fill a magnetized sheath which covers a large area of the target surface. These electrons form a reservoir of energy which is eventually transformed to ion motion without ever penetrating to the interior of the target.

On physical grounds the new model is plausibie, and there is much exferimental evidence to support it (cf. [3]). Several features of the simulation results

Communications on Pure and Apphed Mathematics. Vol XXXY1. +15-4 ih +19k

Not subject to copyright in the United Stales.

Published hy John Wiley \& Sons. Ine 


\section{Magnetle-Fleld-Induced Surface Transport oil Laser-Irradlated Folls}

D. W. Foralund and J. U. Brackbll

Los Aímos National Labonatory, Los Alomos. New Mexico 87545

(Recetved 2 February 1982)

Eleotrons healed by chaorption of laser eaergy are ahown to generate totense magnetic flelds which rapidiy sprend trom the edge of the laser apot along the target surtace. The telds convectively transport hot elc rous and confine a major traction of the deposited laser erersy in the coroan. Eventually, tists energy is loat to tass-ton blowoff or daposIted at lorge distanoes from the spot. This model qualitatively explains inany experimental obeervatione of thermal-tranport tahlbliton and fat-lod la,s.

PACS numbere: $52.25, F 1,52.50, \mathrm{Jm}$

The generation of masnetic fields when intense laser light is absorbed in a plagma is well known. It has been studied because its inhlbition of electron transport would explain the inferred flux limIts needed to model energy transport in laser-produced plasmas. ${ }^{2}$ Here we report new results from fully self-consicient calculations ir colllsionless plasmas." These show large solf-generated magnetic flelds in the corona which convectively transport $30 \%$ or mare of the absarbed enercy laterally and colllmate the remainder behind the laser spot. 'The results appear to qualttatively explain for the first time from IIrst principles a wide variety of experimental data on fastion loss and lateral trensport at various laser wavelengths.

Coronal magnetic fleld generation by collistonless processes is modeled in the reiatively simple geometry shown In Fig. 1. We conotder a plasma foll $500 \mu \mathrm{m}$ wide in $y$ in a $600-\mu \mathrm{m}-w$ ide oychem with a sharp initial density gradtent in $x$. The deposition by a 10.6- $\mu \mathrm{m}$ lneer at an average absorbed intensity of $5 \times 10^{13} \mathrm{~W} / \mathrm{cm}^{2}$ in a $60-\mu \mathrm{m}$ spot is modeled by accelerating some electrons from 2.5 to about $20 \mathrm{keV}$ down the density gradient in a

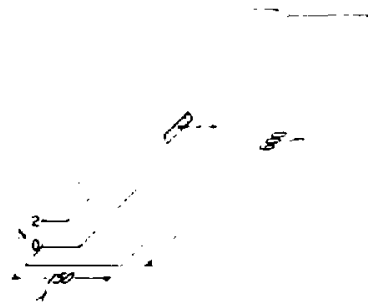

FIG. 1. Diagram showing geometry of madel where the ahwied box is the lagar deposition reglot. The systom is untform in $z$ and the maximum density is twice oritical denatty for $10.6-\mu \mathrm{m} 14 \mathrm{ght}$ $20^{\circ}$ half-angle cone $\mathbf{w}$ ith maximum heating at the center. At the boundary behind the high-density material, heat is absorbed by a cool electron thermal bain. The ion to electron mass ratio is 1836. Thls system is numerically simulated $w$ ith a two-dimensionel, implicit electromagnetic simulation code, VENUS, which solves Maxwell's equatiuns and Newton's low 6 self-consigtently for particle electrons and lons. The behavior with $[(d)-(f)]$ and without $[(a)-(c)]$ self-genernted magnetic tletds is shown in Fig. 2.

Witbout magnetic fields, the electrons accelerated outward return from the aheath back towards the loll. In the $y-v$, phase space of Fig. 2(a), the
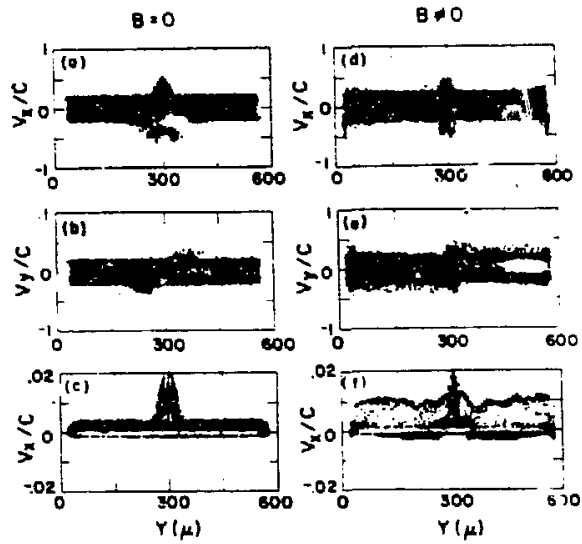

FIG. 2. Reaults of sirnulatlons with a $60-\mu \mathrm{m}$ gpot on a $500-\mu \mathrm{m}$-wide foll at 12 psec. (a)-(c) correspond to $B=0$ and (d) $-(D$ correspond to $B F$. (a) and (d) are electron phsae apree $v$, ve y for all $x$, (b) and (b) are elsctron phase opace $v$, vo $y$ for all $x$, and (c) and (n) are lon phase space $r$, vs $y$ for all $x$. Only electrons with $|v|>0.17 e$ and lone $w i t h|v|>0.00125 e$ are plotted. 


\title{
An Implicit Method for Electromagnetic Plasma Simulation in Two Dimensions*
}

\author{
J. Li BrackBill and D. W. Forslund
}

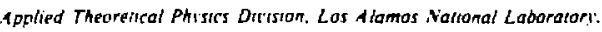

Los Alamos. Ven Mexico 875ts

Received November 13, 1981

\begin{abstract}
A new method for modeling tou-frecuency plasma phenomena is presented The method uses an implicit formulation of the Vlasov-Maxwell equations to relax restrictions on the ime step and mesh spacing so that larger values which correspond to the frequencies and wateiengths of interest tan be used. As a result. the range of iength and time scales accessible 19. plasma simulation is inereased by orders of magnitude. The algorithm. as embodied in a new code VENUS for electromagnetic plasmas in two dimensions. is described. its stabiluts and accuracy analvzed through linear and nontinear analy5is. and its properties, including stepression of the finite grid instabtiti. Illustrated through its application to the Hetbel instabiht!
\end{abstract}

\section{INTRODLCTION}

To extend the reach of nonrelativistic electromagnetuc plasma simulation in two dimensions to longer physical length and time scales. an implicit method has been develeped. The implicit method alters the way the coupled field and particle equations are advanced in time and eliminates many of the constraints on the time and space steps imposed by stability conditions.

As is well known, explicit formulations of the Vlasov-Maxwell equations are stable only for values of the tilne step $J t$ and mesh interval $J x$ that resolve all time and space scales |1,2]. In electromagnetic plasma simulation. for example, one is required to use time steps which resolve light waves and space steps which resolve the Debye length. even when neither radiation nor charge separation effects are important. In many cases, the time and length intervals interest are very large compared with the values of $\mathcal{J} x$ and $J t$ that satisfy the stability conditions. and then many time and space steps are required to integrate over them. This prevents the application of explicit plasma simulation $m$ sthods to many problems |3].

-This work was performed under the United States Deparment of Energy. The U.S. Government: right to tetain a nonexclusive royalty-free license in and to the copyright covering this paper. for governmental pupposes, is acknowledged 


\title{
Plasma Mechanism for Ultravioiet Harmoulc Radiation Dae 10 Intense $\mathrm{CO}_{2}$ Light
}

B. Bezzerides, R D. Jones, and D. W. Forslund

Lnitersity oj Califomla, Los Alamos National Laboraton, Los Alamos. Neu Mexica $875+5$

\author{
(Reootved 18 Jun 1981 )
}

\begin{abstract}
A theoreticel explantion is preseatud of some obeervatione from recent CO,-laser experiments by btroduolos a novel meobeniem for harmonlo light emlasion. The theoretlcal model provides new Insight lato the propertles of larco-emplitude waves in the extremely inhomogeneous ebutroament oausad by atrob proale modification of the critIcel-density plasma.

PACS numbars: 52.25.P., 52.35.Ht, 52.35.Mw, 52.70.K工
\end{abstract}

Harmonic gentration in laser-1rradiated plasmas las been the subject of a number $\alpha$ expertmental ${ }^{1.2}$ and theoretical papers.' These atudies have been conitined primarlly to second-harmonic (SH) tmission. The analytic efforts to understand SH have relled on perturbation theory based on the assumption of a weakly nonlinear response by the plasma in which the source current for the SH is due to beats between the first-harmonic lielo. Higher harmonics up to the eleventh harmonic of $\mathrm{CO}_{2}$-laser light have been reported by Burnett etal. The relative efficiency a the emitted lines in this work was a decreasing function of harmonic number, again a result corresponding to a weakly nonlinear plasma response.

A recent paper reported the observation of $\mathrm{CO}_{2}$ harmonic light as high as the 29th harmonic; and more recently, as high as the 46 th harmonlc." The unique feature of these data is the constant relative efficiency of the lines. It is obvlous that to understand these $d: t a$ one must introduce a new approach whicb goes beyond any analysis based on mode coupling and perturbatior theory. Furthermore, thege data are compelliv; evidence for nonlinearity heretofore unexplored in the study of laser-plasma interactions. The purpose of this Letter is to understand some of the properties of this nonlinearity.

Before $w$ e discuss the nonlinear mechanism for the high-harmonic $\mathrm{CO}_{2}$ emisston, let us consider the absurption of the incidet.t Ught. It is generally accepted that the dominant absorption mechnnism of intense $\mathrm{CO}_{2}$-laser light in laser-fusion applications is resonant absorption." Resonant absorption in a fixed plasma density profile is a linear mechanism whereby incident light tunnels from the electromaginetic turning point and excites plasma density oscillations at the critical density, $n_{c}$, where $\omega=\omega_{p}$, with $\omega$ the incident light frequency and $\omega$, the local plasma frequency. respectively. Throughout the pulse time of the laser the plasma density is certainly not $\mathrm{f}$ ixed and can develop a sharp plasma boundary as a reoult of the very substantial pressure of the intense laser light $\left(>10^{16} \mathrm{~W} / \mathrm{cm}^{2}\right)$. The importance of this oteepened plasina density profile will become apparent in what follows.

To underatand the emitted spectrum we start with the radiation lield,

$$
\overline{\mathrm{B}}(\overline{\boldsymbol{x}}, t)=f\left(\frac{\left[\bar{J}^{\prime}\right]}{\mathrm{c} R}+\frac{[\overline{\mathrm{j}}]}{\mathrm{R}^{\boldsymbol{r}}}\right) \times \hat{R} d t^{\prime},
$$

where the aquare brackets denoke retarded time, the current $\vec{j}=-e n \vec{v}$, with $n$ and $\vec{v}$ the electron thir density and velocity, reapectively, and $\overline{\mathbf{A}}$ $=\overline{\mathbf{x}}-\overline{\mathbf{x}}^{\prime}$. Introducing the Lagrangian variables $\overline{\boldsymbol{x}}^{\prime}$ $=\overline{\boldsymbol{x}}_{0}{ }^{\prime}+\bar{\delta}\left(\hat{x}_{0}{ }^{\prime}, \tau^{\prime}\right), \tau^{\prime}=f^{\prime}$, where $\bar{\delta}=l^{\top} d \tau^{\prime} \vec{v}\left(\bar{x}_{0}, T^{\prime}\right)$ with $n_{\mathrm{a}} d^{3} x^{\prime} \times n_{0} d^{3} x_{0}$, we lind for the far field,

$$
\overrightarrow{\mathrm{B}}(\mathbf{X}, t)=-\frac{e}{c^{2}}\left(\int d^{3} x_{0}{ }^{\prime} n_{0} \frac{\hat{R} \times \partial \overrightarrow{\hat{V}} / \partial T}{R}\left(x_{0}^{\prime}, T\right)\right) \text {, }
$$

where $n_{0}$ is the ion density, assumed $i$ ixed, and now $\overrightarrow{\mathbf{R}}=\overrightarrow{\mathbf{x}}-\bar{x}_{0}$ : W We have negiected relativistic corrections, and used $w \delta / c \ll 1$, which requires $v_{0} / c \ll 1$, as we will see, where $v_{0}=e E_{0} / m w$ with $E_{0}$ the incident field intensity. This form for $\bar{B}$ is useful since it shows that only the fluid acceleration, albeit in Lagranglan coordinates, is needed to calculate $\overrightarrow{\mathrm{B}}$. The justification (or the use of Lagrange-transiormed variable 5 for all time, even though hot electrons are pioduced at high incident intensity, follows from the observation that fluid-element crossing does not occur if the full electron pressure is included in the elec tron Euler equation. Except for the integral over the radiating material Eq. (2) is identical to the single-particle result and leads to the well-known Larmor formula Ior the total power radiated.

The acceleration is given by

$$
\frac{\partial \bar{v}}{\partial t}+\frac{1}{2} \nabla t^{3}=-\frac{e}{m} \bar{E}-\frac{\nabla \cdot \overline{P_{l}}}{m n_{l}},
$$

where we have used the relation $\nabla \times \bar{v}=e \overline{\mathrm{B}} / m c$, and $\bar{P}_{\text {, }}$ is the electron-prissure tensor. We now 


\section{REPRINTS IN SPACE PHYSICS}

SINCE 1982 


\title{
Collective Capture of Released Lithium Ions in t':e Solar Wind
}

\author{
D. WINSKE, ${ }^{1}$ C. S. WU, ${ }^{2}$ Y. Y. LI, ${ }^{2.3}$ AND G. C. ZHOU ${ }^{2.4}$
}

\begin{abstract}
The capture of newly tonized ltthium ions in the solar wind by means of e.- 1, omagnetic instabilites is invesligated through linear anaiysis and computer simuiation. Three ,wlavilites, driven by 1 lithium veloctly ring petpendicular to and drifing along the magnetuc field, are considered. The caplure time of the lithum by the solar wind is toughly 10 linear growit times. regardleas of whether resonant or nonresonant modes dominate intually Possible implications of the resuits for the Active Magnetosphere Parnicle Tracer Explorer (AMPTE) mission are disciussed.
\end{abstract}

\section{Istrodiction}

One of the principal iasks of the Active Magnetospheric Particle Tracer Explorer (AMPTE) mission will be the release of lithium atoms in the solar wind in front of the earth's bow shock and the subsequent monitoring of them as they pass into the magnetosphere. A description of the mission. its overall goals, and the spacecraft instrumentation is given by $\mathrm{Kri}$ migis et al. [1982]. A key issue is where to release the lithium to achieve the most efficient transmission into the mag netosphere. The most promising sirategy is to release the lith ium ilose to the quasiperpendicular portion of the bow shock so that its gyromotion will quickly carry it into the down stream region. as has been shown by following single particle trajectories [Brinca. 1984: Decker et al. 1983, this issue Haerendel and Papamastorakıs. 1983]. Because the solar wind condizions are sometumes highly dyamic and unpredictable. it Is also important to know what happens if the lithium ions do not gyrate rapidly through the shock. either because the bow shock recedes away from the release point or the orientation of the interplanetary magnetic field changes to be more nearly aligned with the solar wind. In this case. motion along the magnetic field is also important. and how rapidly the lithium ions are "captured" by the solar wind, that is, brought up to velocty equal to that of the solar wind in the direction paraltei to the magnetic field, becomes an issue

Here we examine this question, in particular with regard to the role of collective effects in the cafture process. The basic picture we propose is that after ionization the newborn lith ium ions form a ring in velocity space perpendicular to the magneuc field (tn the solar wind frame), while driftung relative to the solar wind along the magnetic field. Such a nng beam velocity distribution is unstable to various electrostatic and electromagnetic instabilities. The situation is sımilar to that discussed by $W_{u}$ and Daudson [1972]. Wu et al. [1973] Hartle and $W_{W}[1973]$. and $W_{u}$ and Harle [1974], who considered the problem of the capture of newly created $\mathrm{He}^{+}$ions of planetary orign. In these artucles the authors hypothesized that the newborn tons can excite a variety of wave modes, and consequently, the instablities and ensuing turbulence can

'Los Alamos National Laboratory. Los Alamos. New Mexico.

"Institute for Physical Science and Technology. Liniversity of Maryland. College Park. MD 20742

Maryland. College Park. MD 20792
3 Permanently at Southwestem Institute of Physics. Leshan. Sichuan, China.

"Permanently at Instutute of Space Physics. Chinese Academy of Sciences, Beijing. China.

Copyngh 1984 by the American Geophysical Union

Paper number $4 \mathrm{AOBO2}$

$0148-0227840041-080250500$ result in the collective eapture of the $\mathrm{He}^{-}$ions. Interest in this problem has been revived by the AMPTE project. The purpose of this brief report is to summanze recent resuits. which demonstrate by means of linear stability analysis and com puter simulation that low-frequency eiectromagnetic instabilities due to the newborn lithium tons may play an important role in the capture process.

While there are many paramete is to be considered. we concentrate here on only one the density of the lithium cloud which is highly uncertain. The density may be comparable to that of the solar wind if iouzation occurs very rapidly, say tonization by the gas-plasma interaction process [. Alfien. 1954], or much smaller than that of the solar wind if pho. toionization by solar uliraviolet radiation prevails. In the analysis we take the lithium density as small but arbitrary and examine the results over a wide range of density ratios $110^{-5}$ to $10^{-1}$. It is conceiveable that even larger lithium concentrations could be possible. but in that case we would expect that large local density variations would develop. inialidating the theory we present.

The plan of the paper is as follows. In section 2 we describe the model in detail and summarize the results of the linear analysis for the three instabilities considered. Results of computer simulations used to investigate the nonlinear behavior of the intabilities are then presented. first for an idealized infinite. homogeneous) situation and then for more realistic cases in which the finite extent of the lithium cloud, its expansion in IIme, and interaction with upstream turbulence are considered Because of the length restrictions of this brief report. we limis the discussion to a summary of the major points; the detarls will be presented later in a full length article. In section 3 we summarize the results and discuss possibie consequences for the AMPTE mission

\section{Restils}

\subsection{Madel}

The distribuin, $n$ of newly lonized particles in the solar wind has been described by $W u$ and Duridson [1972]. If $\mathbf{n}=\mathbf{B}_{\mathbf{0}} B_{0}$ is a unit vector along the interplanetary magnetic field $B_{0}$, in the solar wind frame such ions have a parallel velocity $v_{0 \|}=0$ $\left(Y_{s w}-V_{1}\right)$. where $V_{s h}$ is the solar wind velocity and $v_{\text {, is }}$ the newborn ion velocity. The ions gyrate perpendicular to the magnetuc field with a transverse speed $v_{0 .}=\left|\left(V_{s w}-v_{1}\right) \times n\right|$ For simplicity. we take the angle between $V_{5 w}$ and $B_{0}$ io be 45 and neglect $v$, so that the lithium tons form a ring with $b_{i .}=b_{s w} 、 \bar{z}$ streaming relative to the soiar wind with $r_{14}=$ $v_{1}$. As the angle between $V_{54}$ and $B_{0}$ changes, the source of free energy, and thus the character of the instability, changes from being more beamlike when $V_{5 \psi}$ and $\mathbf{B}_{0}$ are more aligned 


\title{
Low-Altitude Image Striations Associated With Bottomside Equatorial Spread $F$ : Observations and Theory
}

\author{
JAMES F. VICKREY
}

SRI Internatianal. Radio Physirs Lahuratars

\section{Michael C. Kelley and Robert Pfaff}

Sithoul af Electrical Engineering. Cornell L nuersit:

\section{S. Robert Goldmas}

Los Alamns Nattonal Laborator

\begin{abstract}
lunuspherte plasma insiabilutes are usually discussed in terms of local paramelers However. beciause electric fields of scale size, $i 1 \mathrm{~km}$ map along magnetic field lines, plasma populations far audy from a locally unstabie repion mas be aifected by the instability process and vice versa. We present observalion of eleciron density vartations in the $F_{1}$ region of the ionosphere at two locations near the magnetic equjtor Ostillitions in electron number density that were confined to a narrow wavelength repame were observed in 3 region of the ionosphere wilh a very weak verucal density gradien! Since magnetic flux tuhe interchunge instabilttes cannot create structure in such an environment we suggest that these are "Images" of instabilutes occurring elseuhere along the magnetic field line. A simple steady state theory of image formation 15 develoned that is in good agreement with the observations. Moreover this theory predicls a scale sice dependent "eftective deffusion" prucess in the $F$ regon that may dominale over predels a scale sice dependent efrectue difusion prucess in the F regoon that may dominale vever classical ctoss-field diffusion al kilometer seale sizes. Such a seale size dependent difuston process is
reyured to explain recent scinullation observations of decaying equatonal plumes.
\end{abstract}

\section{NTRUDLCTION}

lonusphersc piasma instabilties are olten discussed in terms of local parameters. However, magnelic field lines can link unstable regions to other plasma populations that may affect the esolutiun of the instabiluty process. An example is the role of a conducing $E$ region in difiusive damping of $F$ region structure and in the suppression of electrostatic instabilities [1, olh and Hutrend! 1971. Goldmar et al. 1976. Vickey and K.'llel. 198:]

Another effect most often studied in conjunction with $F$ reuton bartum cloud striatuons is the generation of image striattons in the background medium As discussed in more detait below an image forms at altitudes where the ion gas is compressible. namely. the $E$ and luwer $F$, regions. To date, the existence of images has not been experimentally verified, although computer simulations have predicted their generation [Goldman el al, 1976. Lloyd and Haerendel, 1973].

The data drscussed here come from fixed blas Langmusr probes flown on two rockets launched just off the magnetic equator during equatorial spread $\boldsymbol{F}$ conditions. One, a Javalin soundıng rocket, was launched by NASA from Natal, Brazil, on November 18. 1973, at 21:2 UT [Kelley et al.. 1976. 1979. Cista and Kelle1, 1978]. The second. a Terner-Malemute designated as PLUMEX I, was launched by the Defense Nuclear Agency from Kwajalen Atoll on July 17. 1979. at 1233 UT [S:uszcsewicz el al.. 1980: Rino et al., 1981; Kelley el al. 1982]. We will show that the spectrum of density structure observed in the $F$, layer valley $(160$ - to $200-\mathrm{km}$ altitude) of ine magnetic equator is consistent with that expected from the image" rmalion process (which depends on scale length and

Copynghi 1984 by the American Geophysical Union

Paner number an011\}

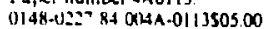

densityl driven by the gravitational Rayleigh-Tayler insia biluty operatung on the bottomside $F$ layer at the equator itsel

\section{Data Preseytation}

A plasma density profile from the dounleg of the Natal rocket flught is eproduced in Figure la with an expanded plou of the $F$ layer valley and $E$ region density profiles in Figure it (upleg data is not avaslable in these flights in the low-altutud: region due to the timing of sensor deploymentl The plasms density profile was extremely structured throughout the fighi Near and just below the $F$ region peak, the lurbulence has been interpreted in terms of the nonlinear arautitional Rayleigh-Tayfor instability (see earlier references) The low. altitude layered $(90-130 \mathrm{~km}$ ) structure is trpical of the equa. corial $E$ region and is not produced by the "1mage" process $O$ interest to the present study are the quast-sinusoidal Auctu. atuons in the $F$, laver valley $t 17010200 \mathrm{~km}$ where the locid zero-order verucal plasma density gradient nearly samishes Because of the finite dip angle at Natal. these fluctuations project along magnetic field lines to the mugnetic equator at heights rangine from $28010315 \mathrm{~km}$. Nolc that this altıtude range corresponds to heights there the local equatorial $F$ region is suspected to be unstabie to the gravitatienal Rayleigh-Taylor process. The geometry is illustrated sche mancally in Figure 2. Of crupse, we do not have simultaneous $F$ region observations directly at the equitor, but it is highiy likely that boltomsice spread $F$ was occurring there also

Another example from the PLUMEX I rocket downleg is presented in Figure 3. Again. quasi-sinusoidal onciliations Here observed in the $F_{1}$ layer valley region at heights that map to the magnelic equator in the altilude range at which the local equatorial spread $F$ fluctuations uere detected The detecior used in the presenl study was not deploved during

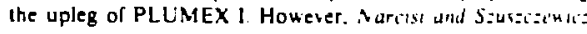
2955 


\title{
EXTENSION OF THE RECONNECTION THEORY OF TWO-RIBBON SOLAR FLARES
}

\author{
R. A. KOPP \\ Inerial Fusion and Plasma Throry Growp. Los Alomas Natianal Laboraton." \\ Los Alemor. NM 87945, U.S.A \\ and \\ G POLETTO \\ Osservatono Astrofsico di Amermi. Larso Enrico Femi S. 5012s Firenze. hah
}

(Received 25 Jenusy; is revised frim B May. 1984)

\begin{abstract}
Atarnact. The mapnetuc reconnection theory for two-ribbon tures and Iare loops hypothesues that the fradual energy release duriag the decay phase is a direct refult of dinipative relasation of the open coronal mapnetic configuration created by an eruptive prominence/coronal transient precursor. This scenano is here developed quantutatively to the point where a rtalisuc comparison with observational data can be attempied Out majo: tefinements are (i) to use an enalyical description of the reeonnecung field geometry specticall chosen to characteruze the spatial acale of the active region where a dare occurs, and (in) to take account of the fact that the velume occupied by X-ray-emutung plasma tho loops generally uncreases with ume dunng the flare.

As a test of the modified theory we have undertaken a new representation of the Skylab observations of the large iwo-ribbon dare of 29 July, 1973 . It is hertby found that the simulaneous inclusion of the above two factors yelds an excelient apeement between the theoretical predietion and observations of the tame varation of hare-plasma energy density: such was not possible within the framework of prevouss wosk alons these lines. The agreememt extends, moreover, to rather early times in the flare history. where the short comings of earlier studies became especially noticeable. This result consututes strong eviderice to supfor previous speculauons that magnetic reconnection may provide the sole energy sousce throughout nearl? the enture lare lifetume
\end{abstract}

\section{Introduction}

The magnetic reconnection theory for the 'decay phase' of two-ribbon flares, as developed originally by Kopp and Paeuman (1976) and subsequently by Pneuman (1981, 1982). Cargill and Priest (1982), Forbes and Priest (1982, 1983a, b), and others. is generally regarded ('́vestka et al., 1980; Pallavicini and Vaiana. 1980) as providing a comprehensive and self-consistent description of the relationships between a wide variety of flare-associated phenomena - älament eruptions, $\mathrm{Hz}$-ribbon brightenings and separations, hot (X-ray) and cool (Hx) llare-loop grouth, and nonthermal panicle generation and storage. Briefly, the theory hypothesizes that a two-ribbon flare is the visible manifestation of magnetic reconnection in the corona above the flare site, the stressed open-field structure within which this reconnection occurs having been created immediately beforchand by a filament activation/disruption and coronal tran sient. The excess magnetic energy of the distended feld is released (rapidly at first and more gadually as the flare progresses) as reconnection allows a lower energ; configuration

- Work performed under the suspices of U.S. Dept. of Energy

Solor Phvics 93 (1984) 351-361. 0038-0938/84/0932-0351501.65

C 1984 by $D$ Resdel Publishine Company: 


\title{
Collisionless Dissipation in Quasi-Perpendicular Shocks
}

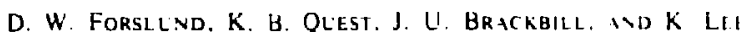

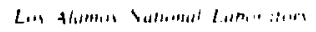

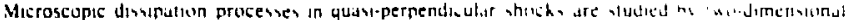

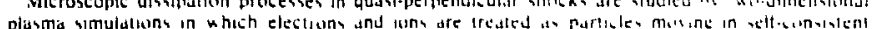

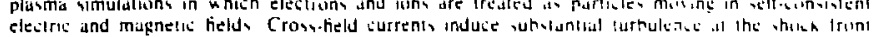

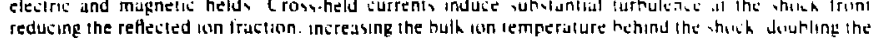

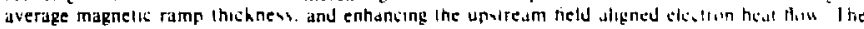

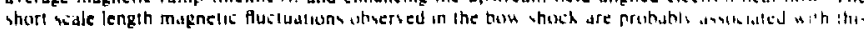
iurbulenice
\end{abstract}

\section{INTRODLLTHON}

Lnderstanding of the dissipation of energy in edlistunlew magnetic shocks has been wught for mant bears. Sulellit: observatluns of the earth's hou shock tlluminate the struciure of collibionless shocks including ion reflection / $/$ timt.

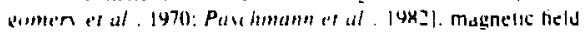

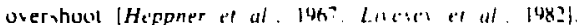

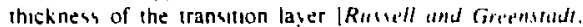

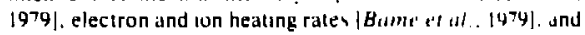

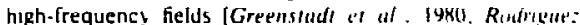
and Gurnetl, 1975, 1976) in the shock lityt itself Compured with these observations. one-dimensional particle vinula.

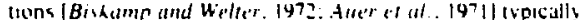
overestumale the number of reflected ion beause cromrield revmlisils is abuent. One-ditrensional hisrid atmula-

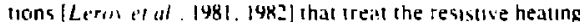
phenomenolugicali: descrube the thacknes of the foot reglon and the overshoot region. However. the mechanism for electron heating is unknown and the proper ramp thicknem is not obta!ned in such models Here. home prelımınary resulis are presented from iso-dimenvional plasma simuldtsons of quast-perpendicular ahoch, that revolte the ion and electron time and space icales. The new results not only reproduce or extend most of the ane-dimensionisl simulation results reported earlier. hut diso allore one to dentif important neu electron and ion dimsipation protesses in the earth , bow shock and to ohaerve upstream tield aligned electron heat flow

\section{SIMLLAIIOA RESLETS}

The hou shock is modeled in (wo dimensiuns hy is new Is deseloped implicil particle-in-cell simulition technique |Brackbill und Forshind. 1982]. Ver; high frequency. $w_{i,}$ osculatiens are elımınated wilhout removing electron inertial and kinetic efects. In the simulation code VENLS Brack. hill and Forshind. 1982!. I wo spatial coordinates and three velocities are treated for each soecies in the self-conbistent. three-component electric and magne:li fields. Numerica dissipation in the code is smaller than the disupation in the shock due to self-consistent collisionle ss collecive effecis.

A magnetic piston 15 used to drive the shock The piston, a tangenual disconunuity which pushes the plasmis towards

This paper in not sublect to $L S$ conr right Publuhed in 19k4 his the Americin Genphysical Linion

Paper numbes 3A1919 the left, is generated at the right houndart bs a trancisine

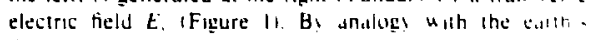

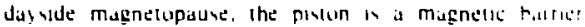

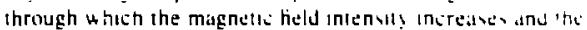
number denull Jroph to erero. In the frume molsing with the

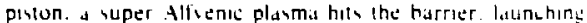

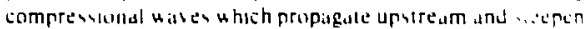

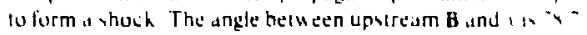
and attou, for some motern of the electrons along the nitu lines ino and oul of the shock.

The simulation paramelers chosen are for $\omega \beta,=2$ plasma with $\beta=2$ or 0.2 and an Alfien maich number of the shock of 4.5 The unis of length in wur figures are upsiream ، "

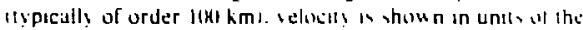
upstreim Alfven speed. and tImt k gisen in term, all the

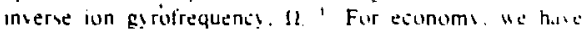
chonen $m$ m. $=\mid(H)$ and w.., $1 !$, = ?1

The direction of the amhient field relatuse to the imulation plane sun strongly affect the 11 per of instiatifities allowed in the code. This can be ured lo dudratige in sludung shoch, For example. in a perpendiculis shock propacatung in the , direction through an ambient magnetic tield in the , dires. lion, the diamagnefis currenth are in the : direction and thus out of the plane of simuldtion thus crom tield surren! instahilites are not atlowed. but pitch angle sillering in-lahilites are. Houcrer. hi roldting the ambient tield out wh the plane. the currents move into the plane atlowing crom-ticld instibillte to oceur. We mike we of this difference 16

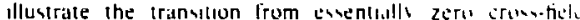
resistusts to the linite lesel self-conomstents produced $h$ crom-held current instihtlites

In Figure 2 se whos phase space plose wath $B$ in the simulation $I . t-y$ plane $\mid B=(i)$ and $T, l=\mid 11$. In Ihis plane

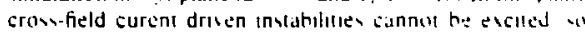
se anticipate a quabl-laminar sho:h vructure with sutstan.

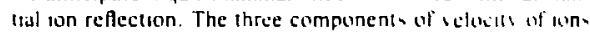

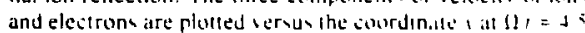
to show the won reflection and hedtung of wn, and elstrum. behind the shock.

At the tlme chosen the shock nowllun is approsimaleh

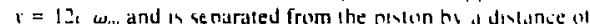

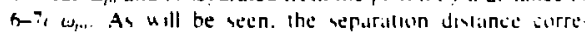

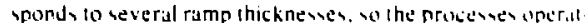
ing withon the ramp may he eximened atpurdtels trun thos near the piston. Addilionilly. the shock separitlun irom ihe left will is large enough so that houndart eftects mall he neglected. 
Collisionless Diselpation Processes In Ruast-Parallel Shockss

K. B. Quest, D. W. Forslund, J. U. Brackblll, and K. Lee

Los Alamos National Laboratory, Los Alamos, Neu Mexico 87543

\begin{abstract}
ARSTRACT. The evolution of collisionless, quasiparallel shocks ( $\theta_{B n}<45^{\circ}$, where $\theta_{B n}$ is the angle between the shock normal and the upstream magnetic field) 1s exarined using tuo dirensianal particle sirulations. Reflected Lona upatreas from the shock are observed with average guiding centar velocity and gytational energy which agree vel velocity and gytational energy which aree wel
wich the prediction of simple gpeculat reflection. wich the prediction of simple opeculat reflectlon.
strong ion heating through the shock raop $1 \mathrm{~s}$ apparently coused by large aplitude whistler turbulence. A flux of supratherwal electrons ig alsn abservad upstreas uith increaged flux obllque to the magnetic fleld direction. Wuch stronger lon heating nccurs in the shock than electron hating. The relevance of this work to the eartits bou shock is discusged.
\end{abstract}

\section{Int roduction}

Collisionless shocks In the solar uind are generated by flareg and by the steepenlng of highspeed solar uind streaws ar large hellocentric diotances (Greensiadt and Fredrleks, 1979), whlie bou shocks surround every planet. Because of the extensive data frow ISEE satellites, detalled comparisons with theory are possible. For exampie, using particle-in-cell plasqa sloulacions wth ldealized boundary condletons and geometries. one car obtain "snapshots" of the Instantaneous velocity distribution functions and the full magnetic lleld praflie at any desited tiue. These may be conpared with analyilc theorles and satellite data to test the understanding of the physics of collisionless shocks.

Here ue present the results of recent twodidensional electromagnetic, fully kinetic simulations of quasi-parallel shocks (5ivilar sirulatlons of quasi-perpendicular shacks are reported in Forslund, et al., 1982). Using an lapleft algorlthm (Brackblll and Forslund, 1982) ve calculate the full electron and 1 on dynarics on large spatial scales (a-ny fon gyroradil) for lang tires (many byroperiods) with ton to mass tatlos of $0(100)$. Thus ve reqove the earller restrictions of one dimensionality (Blskamp and tielter. 1972) and of a resistive tluid treasment of the electrons (Leroy, ec al., [981).

Rerooving thege restrictions allous ug to separace spatially the shock and plston (see belou), to examine electron dissipation, and to observe such feacures as fleld aligned heat flux and electron trapping. This 15 particularly itopartane for quasi-perallel shocks, where motion along the fleld line can result in strong acceleration of the elections chrough the shock transttion Leyer. Tuo dimensionality also allous us to aodel the curbulence due to the cransverse

Copyright 1983 by the tintrican Geuprysical imsth.

iaper jusber 360208.

$0094-8276 / 83 / 003 L-020853.00$ currentg in the shock frone. Flnally, tneluting electron inerclal elfects allous us co propert. treat whistlers gonerated $2 \pi$ quasi-paralle! shocks.

Simulation Mortel and Paraneters

We consider a unfform density plasia in a

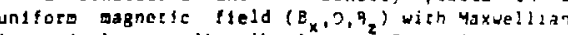
lon and electron distributions at $t$ and - respectively. A magnetic pision is friven into the plagas frore the rlght, launching a shock that ruis ahead of the plston.

In our simulations $6_{8 n} \cdot 37^{\circ}$. che Alfuen yac number is approximarely ${ }^{8 n} 4$ and the upstra. electron 9 is 2 . These values approxloute obserist lons ade during a bou shock crossing by lase : on august 19, 1978, (Gosling et al., 198:). explare the effect of different lon tenperatures. we have held $T_{\text {f }}$ flaed and used $T_{e} / T_{i}=l$ and $1:$. For an upstram electron of 2 , varylng $T$ 't varles the ragnetooonic Mach number frop about ?. (when $\left.T_{e} / T_{1}-10\right)$ to $1 . B\left(T_{e} / T_{f}=1\right)$, The units 0 : length in the flgures, $c / w$, are cyplcally of order $100 \mathrm{ko}$ in the solar wind?' The veloclt" 1 s show in units of the upstreas Alfven specs, and t lae is given in unitg; of the inverse ion girpErequency, $\Omega_{1}^{-l}$, To keep the electron s=ale lengths reasonably resolved, we have chosen $=$ " 100 and $1 \lambda-20$, where $w$ is the electro: plasma frequency. Ali the resulpe below are at coussip then the shock and pision are we:l
separated.

\section{Sirulation Regults.}

In Fig. I we show phase space plots of the inns versus the shock normal coordinate $x$ for the $t$ w

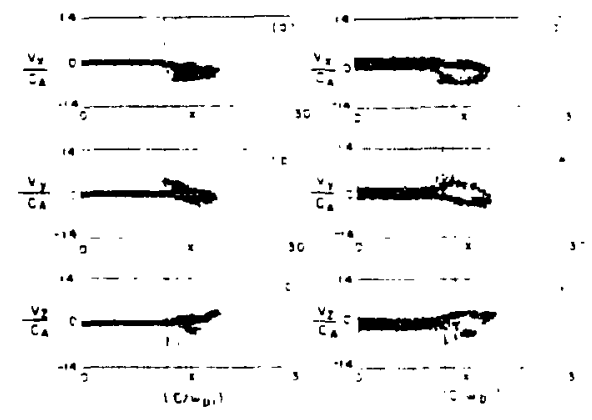

Fig. I Phase space plozs of long ve $x$ for sizula-

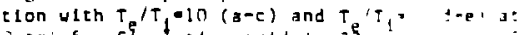

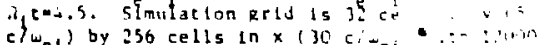
each of electrons and ions and $a t_{1}=$ atep of $4 w_{\text {pe }}-1$ was used. 
REPRINTS IN

DRIVER TECHNOLOGY

AND

FACILITIES

SINCE

1982 
To Be Presented At The 1985 Particle Accelerator Conference TRIUMF, Vancouver, B.C. CANADA

Ion Source Development for the Los Alamos Heavy Ion Fugion Injector, H.L. RUTKOWSKI, S. HUMPHRIES, E.A. MEYER, H. OONA, R. SHURTER, and L. ENGELHARDT, LOS Alamos National Laboratory, Los Alamos, NM 87545--The Heavy Ion Fusion Injector program at Los Alamos is oriented toward producing a 16-beam, high current. $2 \mathrm{MeV}$ injector which wlll gerve as a source for mulc1ple beam accelerator experiments and as a prototype $1 \mathrm{n}$ jector for the High Temperature Experiment (HTE) accelenator. The lon source requirements for this devise are quite stringent. We are reporting on characterization experiments performed in a test stand extractor system, with aluminum spark, pulsed plasma sourses. The pulsed plasma is kept out of the extractor gap with a double grid plasma swltch. Currents are measured with a gridless Faraday probe. Emlttance is measured with a pepper-pot method. The beam itself is imaged with a thin film aluminum fluorescer. We also discuss a multiple beam extractor experiment to study multiple bean effects at low energy.

* Work performed under the ausplees of the U.S. Dept. of Energy.

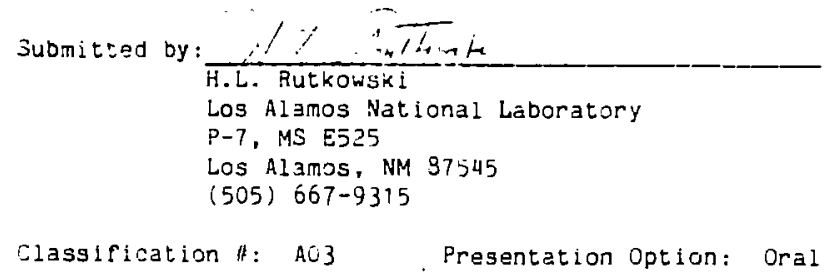


To Be Presented at CLE0 '85, Baltimore, Maryland, May 21-24, 1985

PERFORMANCE OF THE LARGE APERTUR: MODULE OF THE AURORA

KRYPTON FLUORIDE LASER SYSTEM

G. W. York, Jr .

S. J. Czuchlewski

L. A. Rosocna

E. T. Salesky

Los Alamos National Laboratory

Group P-16, MS E543

Los Alamos, New Mexico 87545

Te iepnone: (505) 667-3714

SUMMARY

The Large Aperture Module (LAM) is the final amplifier of the Los Alamos Aurora krypton fluoride (KrF) laser system. ${ }^{1}$ An artist's conception of the device is shown in Fig. l. It consists of a laser chamber with a $1-m \times 1-m$ aperture and a $2-m$ active lengtn whicn is directly pumped by opposing $1-m \times 2-m$ electron beams (up to $675 \mathrm{kV}$ a 20 $\left.\mathrm{A} / \mathrm{cm}^{2}\right)$. Eac' electron gun is driven by two $2.7 \%$ water pulse forming lines (PFL) connected in parallel. The PFLs are eacn $10.7 \mathrm{~m}$ long, giving a 650 -ns pump pulse. Eacn pair of water lines is pulse cnarged by a Marx generator capable of producing up to $1.8 \mathrm{MV}$. A pair of Helmncla coils is provided to prevent beam pincning in the diodes and to ensure uniform pumping in the laser gas volume. The laser chamber can be filled with up to 1.5 atm of an Ar, $\mathrm{Kr}, \mathrm{F}_{2}$ lasing mixture.

To date, tne amplifier nas been operated at up to about $75 \%$ of its rated voltage and preliminary experiments nave been performed to evaluate its performance. An unstadle resonator optical cavity was installed as 
To Be Presented at CLEO '85, Baltimore, Maryland, May 2]-24, 1985

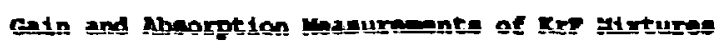

with Bigh ar corcentrations

by

E.7. saleaky

Los Nanos National Eaboratory

P.O. BOx 1663

Los Rlamos, New Mexico 67545

Telephone: (505) 667-5320

and

Wayne D. Kimura

Mathiematical sciences korthwest

2755 Northup way

Bellevue, Washington 98004

Telephone: (206) 827-0460

ABSIRACT

Sma11 signal gain ( $\left.g_{0}-a\right)$ and out-of-band ( $261 \mathrm{~nm}$ ) absorption measurements are made of e-beam pumped KrF laser wirtures ( $A r$ diluent) with

4k-99.6 Kx concentrations. Our computer model agrees with the results. 
To Be Presented at CLE0 '85, Baltimore, Maryland, May 21-24, 1985

Generation and Amplification of Single 5-nis 248-nm

Krypton Fluoride Pulses

by

S. J. Thomas and $M$. D. Burrows

$P-16$, MS E543

Los Aldmos National Laboratory

P. 0. Box 1663

Los Alamos, New Mexico 87545

Telepnone: (505)667-9545

ABSTRACT

A Pockels cell is used to slice a 5-ns pulse from a longer 25-ns krypton rluoride pulse at $248 \mathrm{~nm}$. The 5 -ns pulse is then amplified in a discnargepumped krypton fluoride amplifier witn typical single-pass extraction energies of $200 \mathrm{~mJ}$. The amplified 5 -ns pulse is then used to arive a fusic. laser amplifier chain. 
Presented at LASERS ' 84 , November 27-29, 1984

ENERGY EXTRACTION AND GAIN MEASUREMENTS ON THE LOS ALAMOS LARGE APERTURE KrF :ASER

G. W. York, 5. J. Czuchlewsxi, E. T. Salesky, and L. A. Rosocha

Los Alamos National Laboratory

Los Alamos, New Mexico

\section{SUMMARY}

The Large Aperture Module (LAM) is designed to be the final amplifier of the Los Alamos Aurora KrF laser system. It is a goutle-sided otrect electron beam pumped $\mathrm{kr}$ : amplifier with an aperture of $1 \mathrm{~m} \times 1 \mathrm{~m}$ and an active lengtn of $2 \mathrm{~m}$. In initial tests, this device has been recently successfully operated as a $\mathrm{KrF}$ oscillator, producing an average flux greater than $0.4 \mathrm{~J} / \mathrm{cm}^{2}$ on an array of calorimeters. This 248-nm laser output was produced over the $1-m^{2}$ aperture using a positive brancn, confocal unstable resonator optics set. This resonatir consists of a oair of dielectric coated glass reflectors which form a telescope with a magnification of 2.88 , resulting in an output coupling of $88 \%$. The output window for these experiments consists of a mosaic array of 8 -in. $x$ 8 -in. fused silica windows with a net transmission of approximately $60 \%$, resulting in a delivered optical pulse of $-2.5 \mathrm{~kJ}$. Correcting for window losses, tnis corresponds to an extracted energy from the pumped volume of $>5 \mathrm{~kJ}$ in the 400-ns FWHM pulse at an energy efficiency of $-4 \%$.

Small-signal gain measurements nave also been pertormed at similar operating conditions, and the results will be compared to the predictions of the Los Alamos KrF kinetics code. The data are in good agreement witn the code predictions. Details of the design of the amplifier will be presented. 


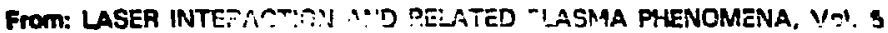

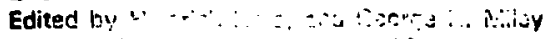

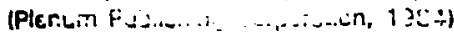

THE AHTARES FACILITY FOR INERTIAL FUSION EXPERIMENTS

- Status amd plans

P. D. Goldstone, G. Allen, H. Jansen,

A. Saxman, S. Singer, and M. Thuot

Los Al amos Mational Laboratory

Los Alamos, NM 87545

INTROCUCTION

In the last decade several increasingly powerful short pulse $\mathrm{CO}_{2}$ lasers have been constructed at Los Alamos Mational Laboratory to investigate the feasibility of $\mathrm{CO}_{2}$ as an inertial fusion driver. The advantage of $\mathrm{CO}_{2}$, a gas laser with high rep-rate capability, electrical efficiency as high as $10 \%$, and scalability to large energies, must outweigh important iffficulties in target physics due to the copious production of suprathermal electrons if $\mathrm{CO}_{2}$ is to be considered a viable driver option.

Since 1978, Los Alamos has used the $10-k J$, eight beam Helios laserl to perform a variety of experiments aimed at elucidating basic laser-matter interaction mechanisms and beginning to determine the scalability of $\mathrm{CO}_{2}$-driven targets to high driver energies. Antares, currently under construction, will be the next $\mathrm{CO}_{2}$ laser used to further the experimental ICF program. Scheduled for operation early in FY-84, the Antares laser is designed to provide $30-40 \mathrm{~kJ}$ in a nominal 0.7 ns pulse, utilizing 24 independently pointable beams. Since Helios is generally limited to $-5 \mathrm{~kJ}$ operation in most target experiments, this will provide a significant increase in the energy available for target experiments, enabling us to study the energy scaling of target interaction phenomena as well as to perform experiments which are energy-limited at Helios. 


\title{
Laser-Initiated vacuum plasma shutters using a dielectric aperture for retropulse isolation
}

\author{
T. W. Sheheen, S. J. Czuchlewski, J. Hyde, and R. L. Ainsworth \\ University of California, Los Alamos National Laborotory, Las Alamas New Mexice 87515
}

(Received 18 December 1981; accepted for publication 23 March 1982)

It is damonstrated that sintered LiF spatial-filter apertures may be used in a vacuum environment of $10^{-0}$ Torr as laser-initiated plasma shutters for retropulse ixolation in high-energy lesser fusion

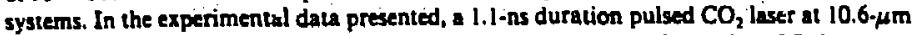
wavelength with an energy of $3.0 \mathrm{~J}$ is used for plasma initiation. A chopped cw $\mathrm{CO}_{2}$ leser tuned to $9.6 \mu \mathrm{m}$ is used as a probe laser in determining the time the plasma blocks transmission of a $\mathrm{CO}_{2}$ laser beam. Both the 10.6 and the $9.6 . \mu \mathrm{m}$ iransmission were measured as a function of pulsed laser fluence on the aperture edge, up to $240 \mathrm{~J} / \mathrm{cm}^{2}$. Blocking times in excess of $1.0 \mu \mathrm{s}$ were observed for an aperture diameter of $800 \mu \mathrm{m}$, for a Gaussian beam with a diameter of $1.2 \mathrm{~mm}$ determined at the $1 / e^{2}$ intensity points.

PACS numbers: $42.60 . \mathrm{Kg}, 42.80 . \mathrm{Bi}$

\section{INTRODUCTION}

In all high-energy laser-fusion systems, an important consideration is the protection of the front-end oscillator and optical components from damage by laser pulses that are reflected back into the laser systems from fusion targets. Any apparatus used in isolating the retropulse should be efficient, with a high forward transmission and low backward trans. mission, have a minimum of complexity fi reliable operation, and for some applications function in a vacuum environment. It has previously been shown that an active shutter using an exploding wire device' may be used as a plasma source at the aperture of a spatial filter to block target-retro reflected light. Metal irises in uir or ambient nitrogen environment also have been shown to produce a laser-initiated plasma ${ }^{2}$ sufficient to "clamp" a retropulse transmission to a low level.

In this presentation, we demonstrate that a $\mathrm{CO}_{2}(10.6$ $\mu \mathrm{m} /$ laser-initiated plasma may be formed at the aperture of $\mathrm{a}$ LiF spatial filter in a vacuum of $10^{-6}$ Torr, of sufficient density and lifetime to block the retropulse from reentering the laser system. The plasma-initiation process is controlled by the fuence incident on the aperture edge of the spatial filter. Blocking times of the $9.6-\mu \mathrm{m}$ probe laser are measured as a function of the incident laser fluences. Blocking times were aiso observed with the $10.6-\mu \mathrm{m}$ pulse laser focused 2.0 $\mathrm{mm}$ of the axis in a radial direction of the cone shaped spatia) filter. With a $1.1-n s$ pulsed $10.6-\mu \mathrm{m} \mathrm{CO}$, laser incident on the LiF aperture, at a fluence level of $63 \mathrm{~J} / \mathrm{cm}^{2}$ on the edge of the $0.7847-\mathrm{mm}$-diam aperture, we have found that a plasma is initiated within the l.1-ns pulse width, producing a small-signal tuansmission of $65 \%$. Within 40 ns. the plasma reaches a critical density of $10^{19} \mathrm{~cm}^{-3}$, sufficient to block the 9.6- $\mu \mathrm{m}$ probe laser. The p!asma persists at or above the critical density for $680 \mathrm{~ns}$; at larger values of edge fluences. tlocking times in excess of $1.0 \mu \mathrm{s}$ are possible. While consideraoie damage to metal irises in vacuum at high fuence levels has been observed, the sintered LiF spatial filter shows only miaimal dumage and no distortion of the aperture after more tian 200 pulses in $10^{-\circ}$ Torr vacuum.

4652 J. Appl. Phye. 53(7), July 1902

\section{EXPERIMENTAL TECHMIQUE}

\section{A. Experimental arrangement}

The present experiments employ up to a $3.0-\mathrm{J} /$ pulse $\mathrm{CO}_{2}$ laser at $10.6 \mu \mathrm{m}$, with a pulse width of $1.1 \mathrm{~ns}$ that is focused on a $\mathrm{LiF}$ spatial flter in a vacuum of $10^{-6} \mathrm{Torr}$ with $\mathrm{NaCl}$ optical components. A continuous-wave (cw) chopped $\mathrm{CO}_{2}$ probe laser at $9.6 \mu \mathrm{m}$ is used to determine the blocking time of the laser-initiated piasma. The beam paths and experimental configuration are shown schematically in Fig. 1 The primary laser beam, with diameter of $3.0 \mathrm{~cm}$, is focused on the spatial filter in the vacuum chamber using a $\mathrm{NaCl}$ lens of $60-\mathrm{cm}$ focal length for an $\mathrm{f} / 20$ system. The input energ; sa.mpled by using a wedge beam splitter, and the energ: uransmitted through the spatial filter are measured w'th py. roelectric joulemeters. Input fuence was varied without changing the beam diameter by inserting calibrated $\mathrm{CaF}$; attenuators into the beam. Calibrated $\mathrm{CaF}_{2}$ attenuators also were inserted into the beam in front of the joulemeters dur. ing calibration. The spatial filter is mounted on a lens holder (show $n$ in Fig. 2) that has vertical and horizontal $|x, y|$ mi. crometer drives that are adjusted from outside the vacuum system. The lens holder and drivers are in turn mounted on an optical rail in the chamber giving $x, y$, and $z$ fine adjustment capability. Provided with these adjustments, the preliminary spatial fitter alignment is obtained using a He-Ne laser beam sent through the optical system, with the spatial filter positioned for maximum transmission. This position is indicated and marked on a video monitor that views the spatial filter with a video camera and telescope. Final alignment of the filter is performed using the puised $\mathrm{CO}_{2}$ laser, attenuated sufficiently to prevent plasma formation or flashing at the spatial filter aperture. The filter then is adjusted for maximum transmission while being viewed using the video sys. tem. The video system gives information on the deviation bet:ieen the $\mathrm{CO}_{2}$ and the He-Ne beam, as well as providing a monitor for observing any asymmetries during plasma formation. Beam spatial filter alignment was accomplished for 


\section{REPRINTS IN \\ MATERIALS \\ TECHNOLOGY \\ SINCE \\ 1982}


Thin Solid Filmr. 107 (1983) 373-378

METALLURGICAL AND mOTECTIVE COATINGS

\section{UNBACKED CYLINDRICAL METAL FOILS OF SUBMICRON THICKNESS*}

DAVID Y. DUCHANE AND GARRY L. BARTHELL

Los Alamas National Latrirators. Los Alomos. NM 87545 : ['S A I

(Recerved Apni 13, 1983; ecoepled Apri 21, 1983)

Implosion experiments often utilize cylindrical thin metal foils. Previously these foils nave been made either from flat sheets, with a seam where the edges joined. or in the form of a composite polymer/metal laminate, in which the plastic film acts as a supportung substrate.

A method has been developed to produce unbacked cyilindrical metal foils of submicron thicknesses. This process utilizes a temporary substrate consisting of a water-soluble polymer film as a base for the electron beam depostion of the metal layer. After formation of the metal foil, the polymer is removed by immersion of the assembly in water. Unbacked metal foil cylinders as thir as $0.17 \mu \mathrm{m}$ with extremely smooth wrinkle-free surfaces have been produced by this technique.

\section{BACKGROUND}

Cylindrical thin metal foils are of interest to a number of researchers conducting pulsed power experiments'. The carliest work in this area was carried out using commercially availabie flat foils. These were simply wrapped around a pair of support rings to produce a foil cylinder $\mathrm{r}^{2}$. The seam at the point where the eviges of the foil were joined was highly undesirable from the stand point of implosion symmetry. I presented a region of essentially zero mass if a butt joint was formed, or an area with twice the inass per unit area of the main body of the foil when an overlap joint was utilized.

To overcome the problems associated with this joint. a technique for producing seamless foils was developed by Kindel et a $!^{3}$ In their process they utilized a cylindrical polymer substrale made according to the method of Grader el al." The product was not a pure metal foil, bui a composite consisting of a polymer film substrate with a continuous and unifom metal overlay. While this seamless polymer/metal laminate was an improvement over :he previous wrapped foils, it presented a number of problems in fabrication and use The polymer film was formed from a noxious organic solvent by a dip-casting process. Because the

- Paper presented at the Inlermasional Conferetice on Metallurgical Couttngs. San Diego, CA. US.A., April [R-22. 198]

$0040-6090 / 83.53 .00$

(C) Elsevier Sequola/Pnnted in The Netheriands 


\title{
Scattering of light by laser fuslon targets with small defects
}

\author{
David E. Cooper, Dau-Sing Wang, and Milton Kerker
}

\begin{abstract}
The extended boundary condition method was used to determ!ne the effect imperfections in small glass and metal shells have on scattered light at $10.6 \mu \mathrm{m}$. The results indicate that imperfections cause a shift in the locations of the minima in the differential scattering curve. a change in the extinction efficiency, and the presence of depalarized components for off-axis orientation of the object. Among these, the presence of and changes in the depolarized component are most sensitive to imperfections. We compute the depalarized scatter from shells with types!. II. and III defects and discuss the potential of light scattering as a character. ization tool for laser fusion targets
\end{abstract}

1. Introduction

Light scattering has long been known to be an ex. tremely sensitive probe of the structure of small particles. ${ }^{1,2}$ This fact suggests that it might be possible to use light scattering to measure specific types of defects on the surfaces of small shells used in the construction of laser fusion targets. Typical shells are fabricated out of either glass, plastic, or $\mathbf{a}$ high-Z metal such as gold. For a sinell to be useful as a target in laser fusion studies, certain well-defined types of defects must be kept below a minimum value. The defects of primary interest. referred to as types I, II, and III, 4 are illustrated in Fig. 1. A type I defect occurs when both inside and outside surfaces of the shell are spherical but not concentric. If either surface is ellipsoidal, we have a type II defect, and a local defect, such as a bump or dimple, is labeled type III.

The characterization requirements on a simple glass or metal shell used in a laser fusion target are quite se. vere and are illustrated in Fig. 2. Specifically, types I and II defects must be below $1 \%$, whereas a type III defect must be held to below $0.1 \%$. Technigues cur rently used to characterize shells with such sensitivities include optical interferometry, $x$-ray microradiography. and scanning electron microscopy. While such techniques are quite sensitive, they are rather time-consuming and do not lend themselves to examination of the

David E. Cooper is with L'niversity of California, Los Alamos Na tonal Leboraton: P. O. Box 1663, Los Alamos, New Merico B 7545 D. S. Wang is with McDonnell Duuglas Research Laboratory. St. Luuis. Missauri 33165: ano M Kerker is with Clarkson College of Technology. Potsdam. New York 13676

Recelved II June 198:

0003-6935/83/010087-12301 (m/0

c 1983 Optical Suciets of America large numbers of targets that will eventuaily be required for a power generating station. Light scattering of fers to be both a sensitive and fast technique of defect detection as well as being considerably easier to implement than the techniques now in use. It is, therefore, of in. terest to determine what effect a defect has on the light scattered by a glass or metal shell.

This paper presents the results of computer studies to determine the effect of types I. II, and III defects on the light scattered by small spherical shells. Although computations were actually carried out in both the op. tical $(0.5 \mu \mathrm{m})$ and IR $(10.6 \mu \mathrm{m})$ regions for both glass and metal shells, only the latter will be presented here since, for the same size parameters, there were only minor differences. The size parameters studied ranged from 5 to 30 . The computer code used in these studies relies on the extended boundary condition method (EBCM) and will be briefly discussed in Sec. II. The complexity of these calculations is substantial, requiring as much as 30 min of computation time on a Cray-1 computer at the largest size parameters studied. Section III presents the results of the calculations, and Sec. IV discusses the feasibility of using light scattering to detect defects of interest on small shells, presents a novel detection scheme, and discusses some difficulties that yet need to be overcome.

\section{If. Theory}

The scattering calculations use the EBCM, which was first developed by Waterman ${ }^{6.7}$ originally for conducting objects, and was then applied to multilayered objects. . $^{-10}$

In this approach, we utilize the spherical harmonic expansions for the incident fields, the scattered fields. and the fields inside the object in conjunction with boundary conditions at the surfaces between adjacent layers to obtain a system of linear equations. The unknown expansion coefficients of the scattered fields are 


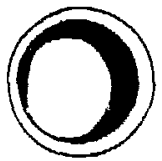

TYPE ।

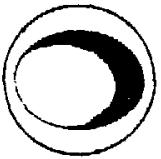

TYPE ॥

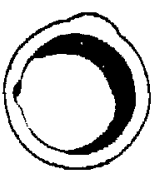

TYPE \#II

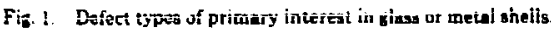

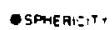

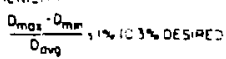

- wall thickness UnIFgami"t

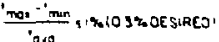

- suarace simothafss

IOE FECT DIAM N'Jro

It: cir

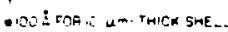

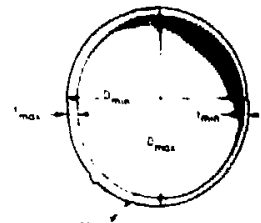

Fig. 2. Characterization requirements on sheils uned in lase: fugion ingets.
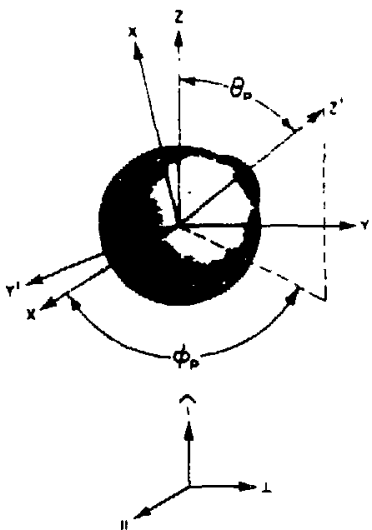

Fig. 3. Geowetry ued in the scattering calculations.

obtained in terms of the known expansion coefficients of the incident fields by directly solving these linear equations. Details of the development can be found elsewhere.11

In this paper we are interested in determining the scattering by a slightly nonspherical glass or metal shell. Since a shell with either a type I, II, or III defect is a body of revolution, we may apply the techrique of using two sets of coordinates in the scattering calculations to take advantage of the fact that the EBCM is particularly efficient for arisymmetric objects. As depicted in Fig. 3 , for a shell with a type III defect (madeled as a hemi. spherical bump in this paper), the two sets of coordinates used are the BODY frame $\left(x^{\prime} y^{\prime} z^{\prime}\right)$ and the LAB frame (xyz). The two sets of coordinites are related by angles $\theta_{p}$ and $\phi_{p}$. In the BODY frame the $z^{\prime}$ axis is oriented along the defect symmetry axis. In the $\mathrm{LAB}$ frame $(x y z)$, the $x-z$ plane is defined as the scattering plane, and the $z$ axis is parallel to the direction of inc1. dence. The components of the incident and scattered fields which are parallel to the $x-2$ plane are defined as the horizontally polarized components, and those componento perpendicular to the $x-z$ plane are defined as the verticaliy polarized components. The major portion of the scattering calculation is performed in the BODY frame, and the solutions are thon transformed into the LAB frame. Using the relations between the two coordinate systems, the scattering calculations can be carried out for any specified orientation of the object and hence location of the defect with respect to the in. cident wave.

The formulation of the EBCM begins with a set of integral equations which relate the fields in different regions:

$$
\begin{aligned}
& \left.\begin{array}{r}
E^{3}(r) \\
0
\end{array}\right)=E^{\prime}(r)+E^{\prime}(r)-E^{\prime}(r) \\
& +\Gamma \times \int_{a_{7}}\left[H_{2} \times E d\left(h_{3} r^{\prime}\right)\right] \cdot \bar{G}\left(k_{3} R\right) d j^{\prime} \\
& -r \times r \times \int_{n, 1} \frac{1}{j \omega_{3}}\left(n_{3} \times \mathbf{H}^{3}\left(h_{3} r^{\prime}\right)\right.
\end{aligned}
$$

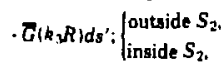

$$
\begin{aligned}
& \left.\begin{array}{r}
E^{2}(r) \\
0
\end{array}\right]=r \times \int_{x_{2}} 1-\lambda_{2} \times E^{2}\left(k_{2} r^{\prime}\right) \cdot G\left(k_{2} R\right) d s^{\circ} \\
& -r \times r \times \int_{12} \frac{1}{j \omega \ell_{2}}\left[-n_{2} \times \mathbf{H}_{-}^{2}\left(h_{2} r^{\prime}\right)\right] \cdot \vec{G}\left(h_{2} R\right) d s^{\circ} \\
& +r \times \int_{t_{1}}\left|h_{3} \times E^{z}\left(k_{2} r^{\prime}\right)\right| \cdot \vec{G}\left(k_{2} R\right) d s^{\prime} \\
& -r \times r \times \int_{11} \frac{1}{j \omega t_{1}}\left[H_{1} \times B_{*}^{z}\left(h_{2} r^{\prime}\right)\right] \\
& \text { - } \bar{C}\left(k_{2} R\right) d s^{\prime}: \text { between } S_{1} \text { and } S_{2} \text {. }
\end{aligned}
$$

where $S_{2}$ is the outer surface of the shell., and $S_{1}$ is the inner surface, $k_{3}, k_{2}, \epsilon_{3}$, and $\epsilon_{2}$ are the wave numbers and the dielectric constants in the surrounding medium and in the shell, respectively. $G(\mathbf{k R})$ is the Green's dyadic for unbounded space, and $R=\left|\mathbf{r}-\mathbf{r}^{\prime}\right| . \mathbf{E}^{i}$ is the incident electric field. $\mathbf{E}^{s}$ is the scattered electric field. $\mathbf{E}^{3}$ and $\mathbf{B}^{3}$ are the total fields outside the object, and $\mathbf{E}^{2}$ and $\mathbf{H}^{2}$ are the fields in the shell. Additional relations between the fields are obtained by applying the boundary conditions at the surfaces $S_{1}$ and $S_{2}$, which require the tangential components of the fialds to be continuous, i.e.,

$$
\left.\begin{array}{l}
A_{2} \times E_{+}^{3}=A_{2} \times \mathbf{E}_{-}^{2} \\
A_{2} \times \mathbf{H}_{+}^{3}=A_{2} \times \mathbf{H}_{2}^{2} \\
A_{1} \times \mathbf{E}_{+}^{2}=A_{1} \times \mathbf{E}_{-}^{!} \\
A_{1} \times \mathbf{H}_{2}^{2}=A_{1} \times \mathbf{H}_{1}
\end{array}\right\} \text { on } S_{1} .
$$

The electric field of an incident plane wave can be expanded as 


\title{
Vacuum deposition of high quality metal films on porous substrates Barry L. Barthell and David V. Dichane
}

\author{
Los Alamos National Loborotory Las Alamos. Nieu' Mexico 8754 !
}

\section{|Received 25 September 1981; accepted 8 December 1981 |}

\begin{abstract}
A composite mandrel has been developed consisting of a core of low density polymethylpentene foam overcoated with a thin layer of film-forming polymer. The surface tension and viscosity of the coatung solution are important parameters in obtaının a polymer film which forms a continuous, smooth skin over the core without nenetrating into tre foam matrix. Weter soluble film formers with surface tensions in the sange of $45 \mathrm{dyn} / \mathrm{cm}$ and minimum viscosities of a few hundred centipoises have been found most satisfactory for coating polymethylpentene foam. By means of this technique. continuous polymer fims with thicknesses of $10-20 \mu \mathrm{m}$ have been formed on the surface of machined polymethylpentene foam blanks. Aluminum has been vacuum deposited onto these composite mandrels to produce metal films which appear smooth and generally defect free even at 10000 times magnification
\end{abstract}

PACS numbers: 81.15.Ef

\section{INTRODUCTION}

The substrate plays a key role in the fabrication of thin metal films by vacuum deposition. since any defects or irregulari. ues in the surface of the substrate tend to be reproduced in the film ' Further, if a freestanding film is to be produced. the sibstrate $m \cdot d s t$ be removed after deposition of the fitm material This can readily be accomplished with parting agents when films are formed as flat sheets of moderate size, but when they are to be made with closed shapes such as cylinders or spheres. removal of the mandrel is much more difficult in these cases mandrels are generally removed by leaching processes w hich ma! be very ume consuming $\mathrm{Me}$. chanical agitation is ofien employed in the leaching process. but this poses a nsk to the integnty of the thin film ln addi. ton, the leaching fluid itself must be carefully chosen. Metal mandrels require lechants which may be corrcsive. difficul to handle, and prone to attack the thin film itseif if param eters such as temperature and $\mathrm{pH}$ are not carefully con trolled. Polymenc mandrel matenals, while soluble in or ganic liquids which pose no danger to the deposited meta film. are usually slow to dissolve and often swell noticeably pnor to becoming completely fluid. Such swelling can de stroy the deposited shell.

The ultimate gaal af the wark repored herein is the development of a composite mandrel consisting of a core of low density, open cell. polymeric foam, overcoated with a thin layer of a smeoth polymer film. Such a mandrel would have a microscopically smooth surface, an extremely high sur. face-to-mass ratio, and an effective void volume approaching 95\% These qualities would largely obviate many of the problems associated with the fabrication of metal-walled fusion targets. The smooth surface would permit the deposi* tion of metallic films of good strength and uniformity Leaching would be facilitated by the high surface-to-mass ratio, or could possibly be eliminated enitrely by simply filling the voids with fuel and leaving the foam core as a perma. nent structural support.

Coating of polymer foams has been practiced in the plas. tics industry for a number of years, but microscopically smooth fintshes have not generally been among the goals of coatung processes: Such surface smoothness as has been achieved often involves the use of fillers and relatively thick coatings :

It has been the primary object of this work to develop coating materials which will form continuous thin films on the surface of a lou densily. open cell foam without penetrating significantly into the body of the foam structure. As will be show $n$ belou, the surface tension and viscosity of the coat ing solution are important factors in the success of this technique.

\section{EXPERIMENTAL}

The polymer foam used in this work was obtasned by a process recently developed at the Los Alamos National Lab. orator: "It consists of polym zthylpentene, and is onginally produced as a filled matenal For these expenments, the filled foain was firs machined into hollow cylinders $23 \mathrm{~mm}$ long and $13 \mathrm{~mm}$ in diameter. The filler was then leached out to yeid a mandrel core of lou densiry labout $0.05 \mathrm{~g} / \mathrm{cm}^{\prime}$ '. open cell, pure hydrocarbon matenal. At this stage of the process. the surface of the foam was macroscopically smooth but microscopicaily rough and porous with an average cell size of about $25 \mu \mathrm{m}$.

In each trial the coaung solution was applied to the surface of the foam by lowenng the core into the coating liquid on a rod extended through its center until it was totally im. mersed. and then withdrawing it at a constant rate of about $15 \mathrm{~mm} / \mathrm{min}$. The excess coating fluid was allowed to drain from the surface while the sample was suspended vertically.

The samples were dryed in a laminar air fiou hood 10 minimize dust contaminanon After dring. each sample was minpected visually and by scanning elect ron microscop! iSEM Aluminum was vapor deposited onto samples of foam coated with the most promising formulations. and the aluminized surfaces again examıned by SEM

The coating solutions were prepared by dissolving polymers known to be good film formers in water or methanol. The materials and concentrations were chosen to give solu- 


\title{
Automatod computer analyais of $x$-ray radlographe greatly facilitates measurement of coating thlekness varlations in laser fualon targets
}

\author{
D. M. Stupin, K. R. Moore, G. D. Thomes, and R. L. Whitman
}

Las Alamas Narional Leboralory. University of Califormia. Las Alamos. New Mexico 87545

(Received 20 October 1981; sccepted 12 November 1981)

We have built an automated system to analyze $x$-ray radiographs of laser fusion targets which greally facilitates the detection of coating thickness variations. Many laser fusion targets require opaque contings 1 to $20 \mu \mathrm{m}$ thick which have ber- 0 osited on small glass be!loons 100 to $500 \mu \mathrm{m}$ in diameter. These contings must be uniformly thick to $1 \%$ for the targets to perform optimally. Our system is designed to detect variations as small as $100 \AA$ in $1 . \mu \mathrm{m}$-thisk coatings by converting the nptical density variations of contact $x$-fay radiographs into coating thickness variations. Radiographic images are recorded in HRP emulsions and magnified by an optical microscope, imaged onto television camera, digitized and processed on a Data General S/230 computer with a code by Whitman. After an initial set-up by the operator. as many as 200 targets will be sutomatically characterized.

PACS numbers: $68.55 .+6,67.70+n, 52.50 . J m$

\section{INTAODUCTION}

We have built an automated system to analyze $x$-ray radiographs of laser fusion targets at the Los Alamos National Laboratory, which greatly fucilitates the detection of coating thickness variations in these targets. The Microradiographic Analysis System (MIRAS - from the Spanish look) is sensitive to $1 \%$ variations in conting thickness for nonconcentricities and $2 \%$ variations for elliptical defects, and, therefore, meets the requirements imposed by target designers for these two types of defects. We huve proven the accuracy and sensitivity of this system by direct comparison with data caken with a flatbed microdersitometer and optical interference measurements. The on-line processing features of MIRAS give fast turn-around time for the analysis of the targets and the automated features of the system mean that the operator is not required to devote his attention full-time to the unalyses.

Laser fusion targets are typically hollow glass microballoons 100 to $500 \mu \mathrm{m}$ in diameter, coated with 1 - to $20 \mu \mathrm{m}$ thick layers of various plastics and metals. While the glass microbalioons are trensparent, it is uaually not practical to measure the conting uniformity or the conting thickness with optical tectniques, because the coatings are usually opaque. However, the uniformity of the microballoon wall and the subsequent coatings must be stringently controlled to guarantee the performanc: of the targets. X-ray ridiography has been shown to be effective in detecting target defects in opsque and transparent coatings, ${ }^{1-0}$ and we are developing the most sensitive methods to find these defects with $x$ rays.

Coating thickness variations are detected by measuring $x$ ray transmission through the targets as shown in Fig. 1 . $X$ rays incident from the top of the figure pass through the asymmetric microballoon and expose the film underneath the microballoon. However, the intensity of the $x$ rays is attenuated exponentially by the amount of the material they pass through. Hence, the $x$-ray fiux to the center of the balloon is not attenuated as much as the flux near the left or

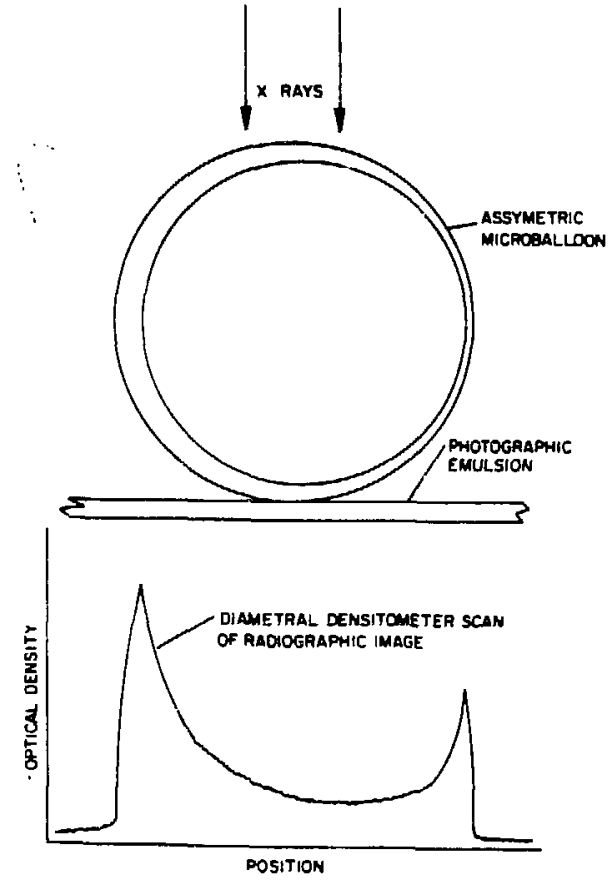

FIC. I. Conting thickness vanuttons are detected by measunng $x$-ray t. uns. mission through the targets The imates are recorded in photographic emulsions and the optical densities of the emuissons are a mesure of the tfuns mitted $x \cdot r a y$ hux. Therefore, coating thickness vanations are detected es varations in the optical density In this example, an asymmetric microballoon gives an unsymmetre optical density plot. 


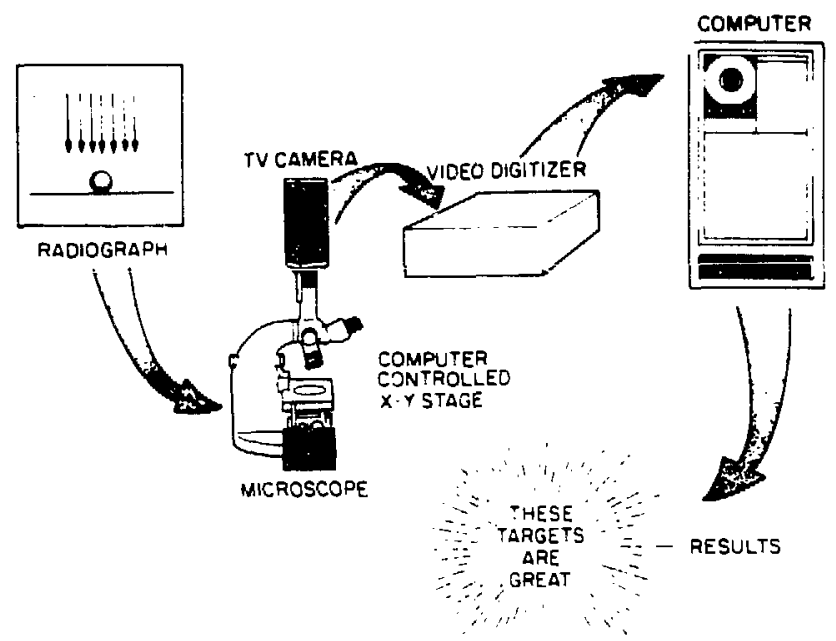

Fic 2 Miras uses a television camern to digi. tize fadiographic images Contact microra. dogranhs are placed on the computer-con. rolled $x \cdot y$ stage and viewed through the microscope by a television camera The udeo images are digitized and stored in computer memon where an analysis program caliu. lates the sizes of defectin and pants the resulis

right edge when the optical densily of the radiographis im. age is plotted along the image diameter, as shown at the bottom of the figure, one typically gets cupshaped plots. Fur. thermore, because of the asymmetry of the nicroballoon. the cup rm is higher on the left-hand side of the plot that 1 is on the right-hand side. This asymmetry in the optucal density plot can be detected by a computer and quantified

\section{APPARATUS}

MIRAS uses a television camera to digitize the radiographic tmage of a target as shown in Fig. 2. First, a contact microradiographic image ts made in photographic emulsion with an $x$-ray source Then this image is placed in MIRAS on the computer-controlled $x-y$ stage and viewed in the mictoscope by a television camera. The video signal is digitized.

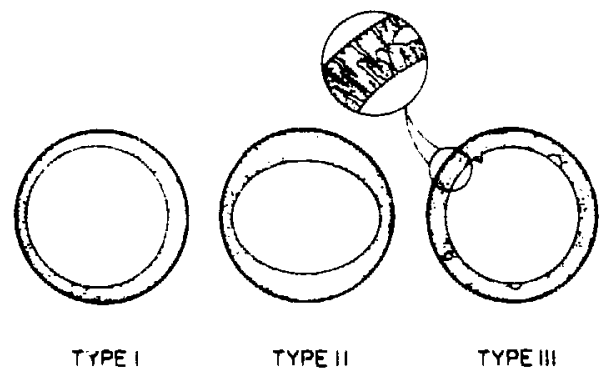

Fic, ? Three ivpes of defects are found in microballowns Typel defecis are

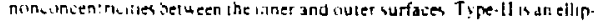

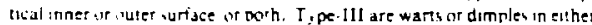

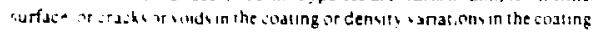

J. Yac. Sel Toehnol, vol. 20, No. A April 1982 this digital data is stored in the computer, and the computer searches the digital data for defects and prints the results of the search. Up to 200 images in a single radingraphic arra: will be analyzed automatically without further assistance after the operator has indicated the location of the images and assigned identification numbers. The computer prints the identification. microballoon diameter. thickness of the coating. nonconcentncity i Type-I defecti, elliptictty :Type. II defect I, and volumes of the warts and pits $\mid T_{y}$ pe-1II defect for each image. A more detailed description of this process is in the Appendix

\section{PERFORMANCE}

MIRAS meets the requirements ai inertial confinement fusion target designers for sensiluvily to Type-I and Type-II defects. We have arbitrarily divided the types of defects typically found in microballoons and coatings into three types which are shown in Fig. 3 The Type-I defect is a nonconcentricity between the outer and inner surface. a Type-II is an elliptical inner or outer surface, or both. and Type-III is a small local defect sucin as a wart or a dimple, en either surface. or a density vanation in the coating. Target designers have estimated that Type-I or Type-II defects smaller than $1 \%$ in $\Delta t / l$ will not degrade target performance, where $t$ is the average coating thickness and $d t$ is the variation about the average. Defects of Type-III must be smaller than $0.1 \%$ in $\Delta t / d$ if they cover an area of $t^{2}$. Whitman ${ }^{2.4}$ has shown that the analysis code in MIRASS is sensttive to $1 \%$ Type-1 defects and $2 \%$ Type-11.

The accuracy of MIRAS is proven by comparison with flatbed densitometer data and optical interference measurements. In Fig. 4 are shown the measured $n$. ncentncities in percent $\mathrm{st}$ it for ten microballoons whis. "re analyzed with data taken with both a flatbed densitometer and with 


\title{
Summary Abstract: A technique for calculating DT content in glass micreballoons from $x$-ray counts
}

\author{
B. S. Jorgensen
}

Universiy of California. Las Aiamos National Laboratory. P. O. Bo= 1003. Los Alamas, Rieu. Mex: o 87545

(Received 18 September 1981; accepted 12 November 19811

PACS numbers: 82, 80.Di

The $\mathrm{x}$-ray counting method' is very useful for determining DT content of glass microballoons (GMBs). A quastititative measurement is complicated. however, by the "ressure- and diameter-dependent self-absorption of the beta particles in the gas and by wall composition and thickness effects on $x$ ray gerieration. ${ }^{2.3}$ We have expe:imentally studied the effects of wall thickness, diameter, and fill pressure on $x$-ray count rate as measured by a $\mathrm{Nal}$ detector and, in addition, the attenuation caussa by a plastic costing on the microballoon. At this time, the effects of glass composition were not investigared. A $5 \% \mathrm{Na}, 12 \% \mathrm{Ca}, 2 \% \mathrm{~K}, 0.03 \% \mathrm{~B}$, and $81 \% \mathrm{Si}$ composition was used in all cases. The results were com. bined in an equation which calculates the fill pressure directly from the $x$-roy count rate by an iterative process.

The effects of varying the wall thickness, diameter, and fill pressure were studied by holding two of the parameters constant and varying the third. Several GMBs were filled by permeation with $50 / 50$ DT for each case. The $x$-ray attenuation caused by a plastic coating (parylene) was found by measuring $x$-ray count rates of individual microballoons before and after coating. The data were plotted as shown in Figs. 1 (a) through (d), and expressions were found for the effects of each variable on the count rate by entering the data on a ctrve-fitting program on a programmable calculator. In each case, a nonlinear, least-squares fit of the data gave a minimum confidence of 0.93 .

The effects on the $x$-ray count rate of the wall thickness, diameter. and fill pressure may be expressed by

$$
\begin{aligned}
& C / V=K_{W} e^{-009 w}, \text { ior } D=D, \text { and } P=P_{.} . \\
& C / Y=K_{0}\left(1.22-9 \times 10^{--} D\right), \quad \text { for } W=W .
\end{aligned}
$$

for $W=W$, and $P=P$,

$$
C /|V P|=K_{P} P^{-0,4}, \text { for } W=W \text {, and } D=D_{r} .
$$

where $I_{5}$ is the counts per second, $V$ is the volume of the microballion in cubic microns, $W$ is the wall thickness in microns. $b$ is the diameter in microns, $P$ is the pressure in standard atmospheres, $K$ is a calibration constant, and the subscript $r$ refers to a reference microballoon.

Combining Eqs. 1, 2, and 3, and solving for $P$ yields

$$
P=\frac{e^{0+16} P^{014} C}{K ! 1.22-5 \times 10^{-4} D, F}
$$

where $K$ is the appropriate combination of $K_{w}, X_{D}, K_{F}$

The equation may be used to calculate the fill pressure of plastic-coated microballoons by including a factor for the $x$ ray artenuation due to the plastic. The attenuation factor is the count ratz of a coated in robalioon $\left|C_{+}\right|$divided by the count rate of the microballoon before it was coated $i C$. As shown in Fig. IId).

$$
C_{T} / C=e^{-00019 T} \text { or } C=C_{1} e^{0.0014 t}
$$

This relationship is valid for coatings frort $U$ - to at least 100 $\mu \mathrm{m}$-thick the upper limit of our measurements). Therefore. the fill pressure of a plastic-coaied microballoon may be cal. culated by

$$
P=\frac{e^{00012} P^{0.14} e^{00030 t} C_{r}}{\kappa\left(1.22-9 \times 10^{-4} D\right)}
$$

The calibration constant can now be found for a specific $x$ ray counter and GMB type by measuring the $x$-ray count rate of a DT-filled GMB and then measuring the fill pressure directly $b$; some destructive technique such as mass spectroscopy Unknown fill pressures can then be calculated using the above equation in an iterative process on a programma. ble calculator. An estimated $P$, which does not need to be a close approximation to the actual $P$, is inserted in the equation and only a few tterations are required to arrive at the fill pressure.

The actual effects derived from first principles are much more complicated. ${ }^{2}$ bur the above corre.lations hold at least for glass microballoons with wall thicknesses varying from 1.5 to $5.5 \mu \mathrm{m}$, diameters from 150 to $600 \mu \mathrm{m}$. and pressures from 10 to $100 \mathrm{~atm}$. The fill pressures calculated using this equation have been compared to the equilibrium fill pressure for mumerous recently filled targets. We have found better that 10\% agreement. Also. multiple calibrations were made via destructive measurements and we again found better than :0\% agreement in the range of pressures and sizes mentioned.

We have found this method to be quick and easy ior assaying the fue! content of glass microballoons to be used for laser fusion targets. With the increasing use of plastic coatings on targets, the factor for $x$-ray attenuation due to the r'astic is extremel: useful since the fill pressure can b: calculated from the $x$-ray count rate of the finsshed target. The effect 5 of varying the glass composition stull need to te stud. ied, since other glass types are frequently used for targets 


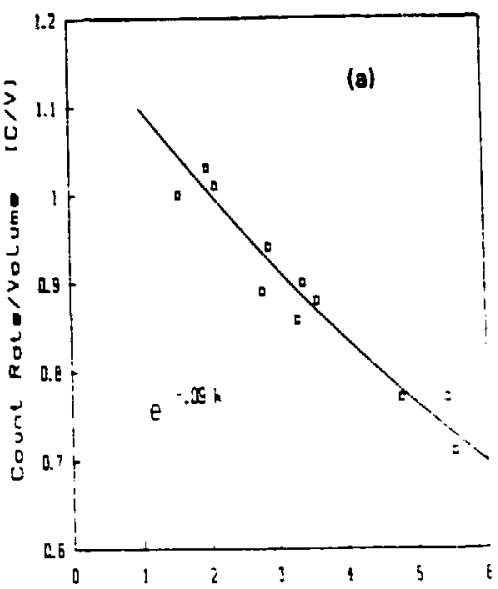

Ho.. Tntorness iterors:

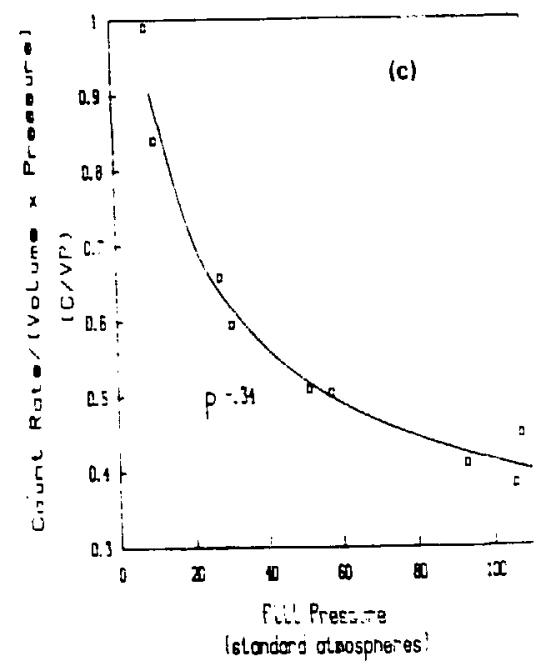

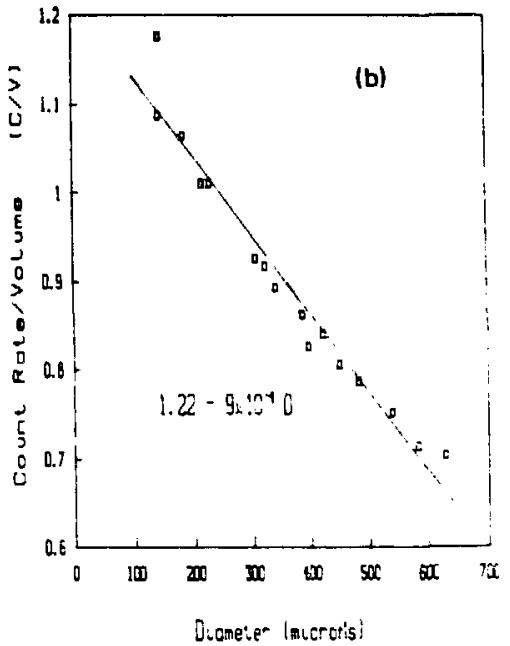

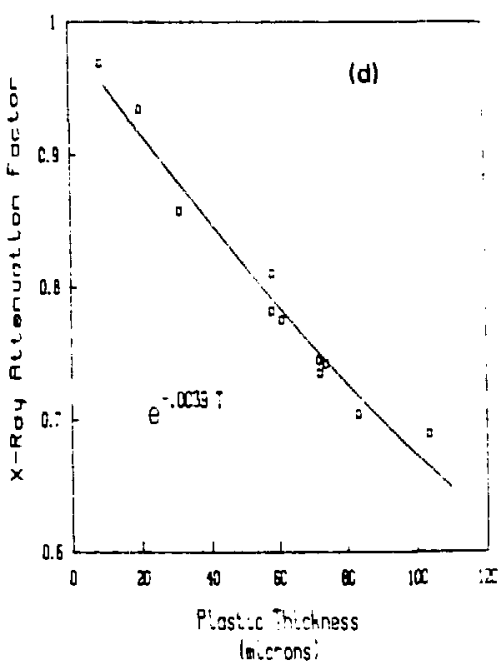

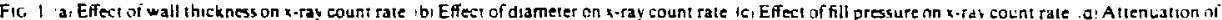
a-rave due to plastic zoatinB

R I Fnes and E H Farmum. Nuct Instr Methods 126. 285।1975 :M M Mueller. 1975 Annual Division of Pl 35 a Physics Meeting of the Amencan Physical Society. St. Petersburg. Flonda 11075. Los Alamos
Report LA-UR-75-2002

1980 Annual Tochnicsl Report, KMS-Fusion. Ine. Ann Artor. Michigan. Sec. 1.3.3.

J. Vuc. Sci. Technot., Vol. 20, No. 4, April 1982' 


\title{
Fabrleation of thin-wrall, fresatanding Inertial confinement fualon targets by chemleal vapor dopoeltion
}

\author{
D. W. Carroll and W. J. Mecreary
}

Los Alamas National Laborotory. Las Alomax. New Mexico 87545

(Received is September 1981; accepted 21 October 1981)

To meet the requirements for plasma physics experiments in the inertual confinement fusion (ICF) program. chemical vapor deposition ICVD in fluid beds was used to fabricate freestancling lungi.ten spheres and cylinders with wall thicknesses less than $5.0 \mu \mathrm{m}$. Molybdenum and molybdenum allos ITZMi mandrels of the desired geometry were suspended in a carrier bed of dense microspheres contained in an induction-heated fluid-bed reactor. The mandrels were fres to foat random! through the bed, and using the reaction $W F_{,}+3 H_{:}^{T_{2} 3 E}-W-6 H F$. very finegrained tungsten was deponsited onto the surface at a rate and in a grain size determined by temperature. gas flow rate. system presture, and duration of the reacuon. After coaung. a portion of each mandrel wavexpoxed by hole drilling or grinding The mandrel was then removed by and leaching. leaving a freestanding iungsten shape Experimental procedures, mandrel preparation. and resulis obtained are discussed

PACS numbers $8115 \mathrm{Gh} .52 .50 \mathrm{Jm}$

\section{INTRODUCTION}

The use of metal foils as inerial confinement fusion (ICF) targets has recently gained imporance in physics experiments applicable to thermonuclear ignition. $1:$ The current requirements for these folls dictate that they must be a specific size and geometry. freestanding, defect-free. less than $5.0-\mu \mathrm{m}$ uniform wall thickness, and be capable of withstanding manipulation durng the fixtunng process. To produce folls satsfying these specincations, a fluid-bed technique in combinstuon with chemical vapor depostion (CVD) was utilized. " Molybdenum or molybdenum alloy (TZM man. drels machined to the desired size and shape were placed in a bed of dense spherical particles. Hydrogen was passed into the bed to begin fludization. This action provided support for the mandrels and caused them to move through the bed in a random manner. After the fluid bed was stabilized at temperature. tungsten hexafluoride $\left(W F_{b}\right)$ was admitted. Using the reaction $\mathrm{WF}_{\mathrm{A}}+3 \mathrm{H}_{2}{ }^{22 \mathrm{~K}} \rightarrow \mathrm{W}+6 \mathrm{HF}$. strong. smooth tungsten coatungs exhibiting fine gran structure were deposited on the mandrel. Subsequent mandrel removsi by acid leaching yielded thin-walled itypicall! 1.5 to 3.0 $u \mathrm{~m}$. freestanding hollow tungsten cylinders and spheres. This paper will describe the apparatus required and the specialized techniques developed to produce such unique tungsten foils.

\section{EXPERIMENTAL}

Hollow cylindncal mandrels were machıned and polished from molybdenum tubing using conventional techniques. Molybdenum was chosen because of its close coefficient of thermal expansion match with tungsien and because it would selectively acid leach without affecting the tungsten coating. Approximately $0.5 \mathrm{~mm}$ wis acided to the lengh of cylinders as allowance for polishing back to the mancirel prior to leaching. Although cylindrical mandrels of various sizes were tungsten coated, the coating process described applies specifically to mandrels $10.0-\mathrm{mm}$ diam $\times 13.6-\mathrm{m} n$ long.

After ultrasonic cleaning, a single molybdenum cylinder wes placed in a bed fluidized by hydrogen. A typical fluid bed was contained within a graphite ccater and consisted of $8 \mathrm{~cm}$ ' of $\sim 350-\mu \mathrm{m}$-diam spherical uranium carbide coaled with tungsten. Average bed density was $12 \mathrm{~g} / \mathrm{cm}^{\prime}$. One unique aspect of this process is that by varying bed compos:-

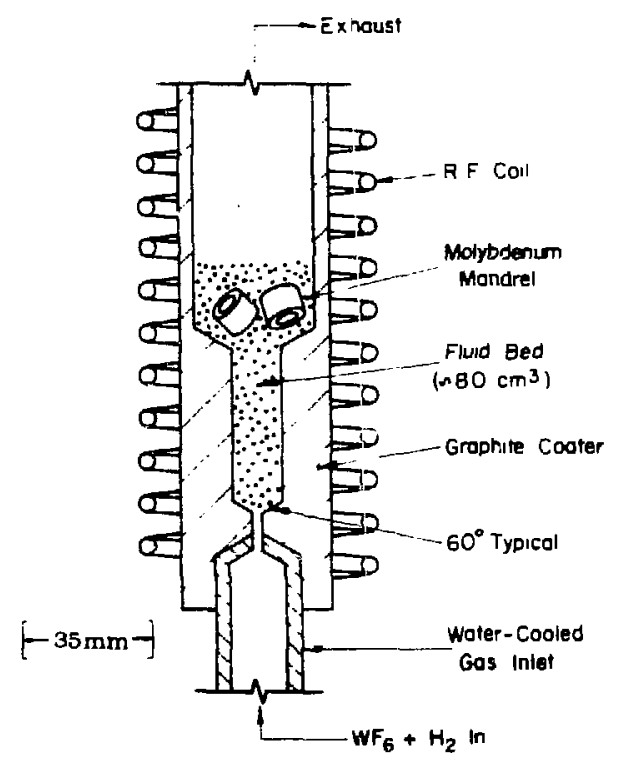

FiG 1 CVD coatıng apparatus, cross section through coster 


\title{
Preparation of multishell ICF iarget plastic foam cushion materlais by thermally induced phase inversion processes
}

\author{
A. T. Young, D. R. Moreno, and . G. G. Marsters \\ University of Colifonia, Los Alamos National Leboralory. PO Bax l603. Los Alamos, NM 87545
}

(Received 21 September 1981; accepted 30 October 1981)

Homogeneous, low-density plastic foams for ICF targets have been prepareo oy thermally induced phase inversion processes. Uniform, open cell foams have been obuined by the rapid freezing of water solutions of modified cellulose polymers with densities in the range of 50-7 $\mathrm{mg} / \mathrm{cm}^{3}$ and respective average cell sizes of 2 to $40 \mu \mathrm{m}$. In addition, low-density, microcellular foams have ben prepared from the hydrocarbon polymer poly(4-methyl-l-pentene) via a similar phase inversion process using homogeneous solutions in organic solvents. These foams have densities from 20 to $50 \mathrm{mg} / \mathrm{cm}^{3}$ and average cell sizes of $20 \mu \mathrm{m}$.

PACS numbers: $82.70 . \mathrm{Rr}, 81.20 . \mathrm{Sh}$

\section{INTRODUCTION}

Several laboralunes have recent ; been invoived in the devel. opment of multishell fusion targets for laser inieraction experiments. :- Initial designs consist of a DT-fuel-filled. goldcoated. hollow glass microsphere centered in the interior of an curer pusher-ablative shell IFig. I. Nonconcentricity between the inner and outer shells must be $2 \%$ or less. Between the two shells is a low- $Z$ cushion layer, which. depending on the particular design, requires denstices from $0.01 \mathrm{to} 1 \mathrm{~g} / \mathrm{cm}$ or more. This cushion layer can be a gas, a small-cell plastic foam, or a full-density plastic.

Multushell fusion targets have been fabricated using a gas uushion layer with a thin plastic film supporing the inner sinell.' Tonsiderable effort has been expended to fabncate low-Z. low-density, microcellular foams for this cushion layer. " " In the study by R.unde and Sione' a cellutose acetate foam w as prepared by extrus; on of an acetone solution of the polymer into a "phase inversion" environment. This process is similar to the methods for making asymmetric, reverseosmosts membranes for wiater desalination. "Finde and Stone were able to produce cellulose acetate foam strands with densities about $50 \mathrm{mg} / \mathrm{cm}^{3}$ and cell sizes of 1 to $2 \mu \mathrm{m}$.

Coudeville et al, ," have reported coating of stalk -mounted giass microspheres with a dextran foam with a density of 50 $\mathrm{mg} / \mathrm{cm}^{\prime}$ and average cell sizes of $\mathrm{l}$ to $2 \mu \mathrm{m}$. Their process involved the rapid cooling of a water-dioxane solution of the dextran polymer to a temperature well below the freezing point of the solvent mixture. The solvent mixture was then removed via sublimation uider vacuum (1.e., freeze-drying to give the polymer foam structure.

In a parallel study at this laboratory, plastic foams have been made from a water solution of the ammonium salt of carboxymethyiceliulose IFigs. 2 and 3 / via this process over a range of densines from $50 \mathrm{mg} / \mathrm{cm}^{3}$ with $2 \%$ m celis 1016 $\mathrm{mg} / \mathrm{cm}^{\prime}$ with $10-\mu \mathrm{m}$ cells. In addition, foams with a density

\section{POLARIS PRIME}

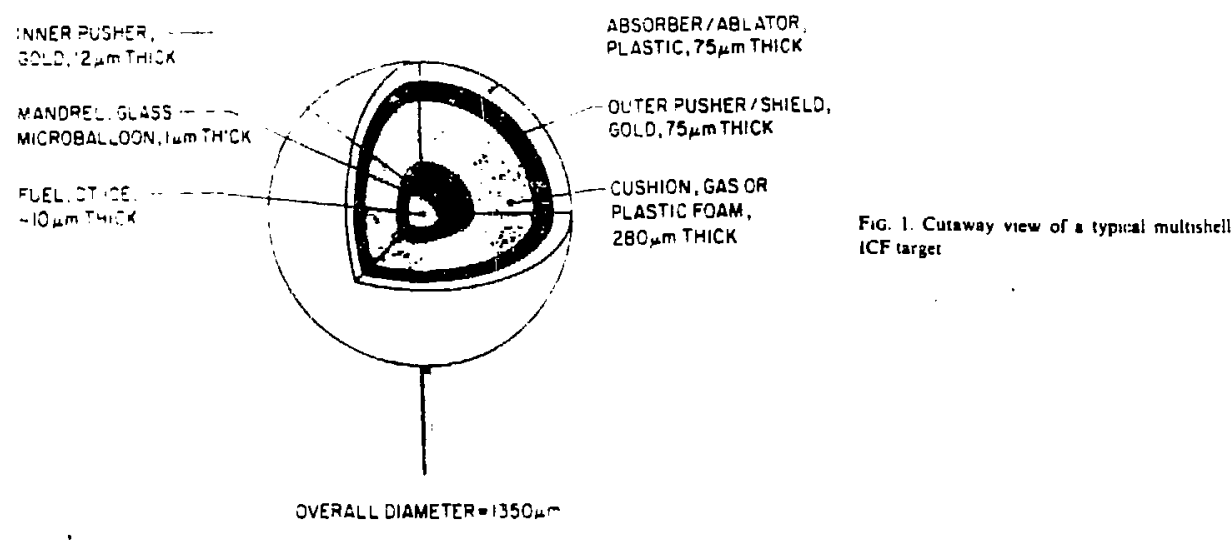

1094 J. Vac. Sel. Tselinal, 20(4), April 1982

$0022-535582.041094-04301.00$

ç 1982 American Vacuum Society 1094 


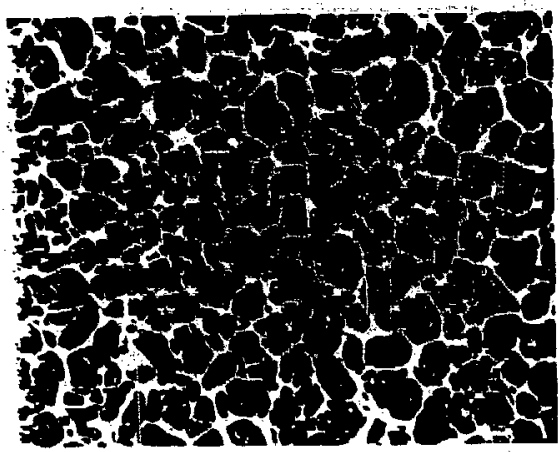

जIG. 2. Caboxymethylcellulose foum; density = $50 \mathrm{mw} / \mathrm{cm}^{\prime}$; average cell vie $=2 \mu \mathrm{m}$.

if $7 \mathrm{mg} / \mathrm{cm}^{3}$ (Fig. 4 ) were prepared from a water solution of nethylcellulose.

\section{THERMALLY INDUCED PHASE INVEASION PROCESSES}

The process employed by Coudeville and in our laboratoy falls into the general category of tivermally induced phase nversion processes. In these phase inversion processes, a homogeneous polymer solution is cooled in order to force separation of the solvent from the polymer to form a continuous polymer matrix in which is dispersed diserete cells of soivent. Removal of the solvent droplets from the matnx generally results in an open cell plastic foam structure where the voids are replicas of the solvent droplets. In a thermally induced process the solvent is selected from a class of solvents which are good solvents at one temperature and poor solvents at a lower temperature. Controlled cooling of a polymer dissolved in this solvent can yield a polymer foam.

During the sooling step. the temperature region at which solvent-polymer phase separation occurs may be defined as

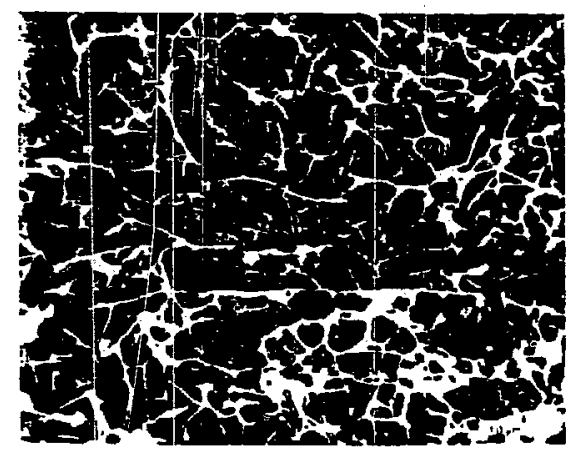

Fig 3 Carboxyme thylcelfulore foam: density $=16 \mathrm{mg} / \mathrm{cm}$ '; average mean free path distanic between rits $=10 \mu \mathrm{m}$

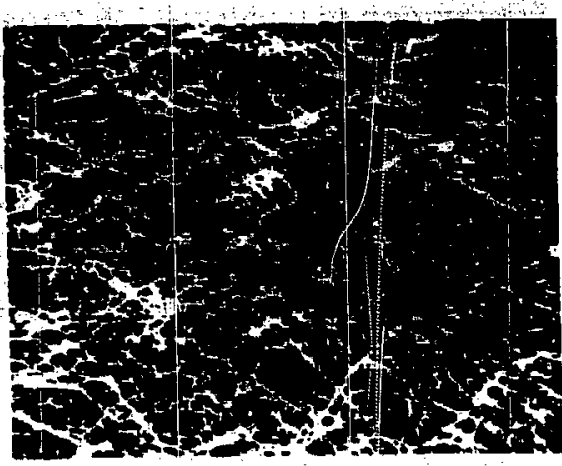

Fic. 4. Methylcellulose foam; densily $=7 \mathrm{~m} \| \mathrm{cm}$; averaje mean free pash distance between rils $=40 \mu \mathrm{m}$.

critical miscibility zone. For polymers having a broad molecular weighi range, the zone may occur over a temperature range rather then at a single temperature.

\section{EXPERIMENTAL}

Since dextran and the chemically modified celluloses contain appreciable oxygen (an undesirable constituent in some target designs), we have studied thermally induced phase inversion foam generation with solutions of pelymers containing only carbon and hydrogen ( $\mathrm{CH}$ ). We have been able to develop $\mathrm{CH}$ polymer fosms with densities in the range of $50 \mathrm{mg} / \mathrm{cm}^{3}$ and cell sizes frorn 10 to $30 \mu \mathrm{m}$. In addition, we have developed a process whiteby these delicate foams can be readily fabricated into detirable configurations via conventional micromachining te:shniques.

Poly|4-methyl-1-pentenel, which is manufactured by Mitsui Petrochemical Co. in Japan under the trade name "7PX." is a linear CH polymer having a density of 0.83 $\mathrm{g} / \mathrm{im}^{3}$. The polymer is relatively insoluble in most common organic solvents, and only recently have solvents been published for TPX."

We have found tha: homogeneous TPX solutions can be obtained with particular solvents by extended heating at elevated temperatures. Such solutions are then cooled at a rate and to a temperature such that nonequilibrium liquid-liquid phase separation is initiated. As the solutions are cooled, it is necessary that no mixing or any other form of agitation be

T INI I. L. Solventw u'ed in prepuring TPX form

\begin{tabular}{|c|c|c|}
\hline Fitrent & $\begin{array}{l}\text { Ph: wiul vtale } \\
\text { st rinm teniperature }\end{array}$ & 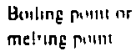 \\
\hline Dipheri! Imethane & Llyuld & $26+4^{\circ} \mathrm{C}$ \\
\hline Phenyl ether & Liyuid & $250^{\circ} \mathrm{C}$ \\
\hline Cy,luhe w thenetene & Lıguid & $2.30^{\circ} \mathrm{C}$ \\
\hline 1-1'hethylistalle & Lı4uid & $261^{\circ} \mathrm{C}$ \\
\hline Bihen/!l & Silid & $190^{\circ} \mathrm{C}$ \\
\hline Hexdmélhỵ/hen'tets & Sollu & $162^{\circ} \mathrm{C}$ \\
\hline
\end{tabular}

J. Vac. Scf. Techiıol., vol. 20, No. 4, April $19 a 2$ 
REPRINTS IN

ICF SYSTEM STUDIES

SINCE

1982 


\section{IMPROVEMENT OF THE TWO-TEMPERATURE RADIATIVE TRANSPORT MODEL}

THOMAS J. MCCARVILLE TRW' Energy Development Group One Space Park. Rl/2128, Redondo Beach, Californo 90278

GREGORY A. MOSES and GERALD L. KULCINSKII

Untversity of $\boldsymbol{\mu}$ 'isconsin-Madison, Depariment of Nuclear Enfineprimg

Is00 Jahnson Drive, Madison, Hisconsin 53706

IHOR O. BOHACHEi'SKY Los Alamos A'ational Latoratory

Los Alamos, New Mexica 8754s

Received November 12, 1982

Accepied for Publicanon February 28, 1983

The frequency dependcnce of a thermal radiation field complicates the computation of radialive energy transport in optically thin media because the spectrum may be uncaupled from local thermodynamic conditions. A model for combining the effect of the frequency dependence into a radiation temperature chosen to represent the temperature of both local and nonlocal emitting regions is described. The derivid equations are much easier to solve than the frequencvdependent equations and can be applied to a broud ciass of problems. The equations are used to invesirgate the response of a gas in an inertial conjinement fusion 'ICF) reaction chamber to torget explosions. The response is compared for ambient densittes of $1.77 \times 10^{18}$ and $1.77 \times 10^{17}$ arom $\mathrm{cm}^{3}$. The error in using the brighttiers temperature instead of a color temperature to evaluate the opacmes is illustrated. An analyfic analysis show's the cooling wave observed from energy releases $>10^{\text {lh }}$ erg will nol occur in an ICF cauly. This is confirmed by the numerical calculations.

\section{INTRODUCTION}

There are a variety of models for computing thermal radiation transport. The modsl that best applies to a particular application depends on the opasiry of the transport medium. For exariple. if the mean-frep-paths of emitted photons are nucil smaller than he distance over whish the temperature changes significantly, then the radiation field is isotropic and the frequency spectrum is determined by the local temperature. Under these circumstances, the dependence on angle and frequency can be integrated out of the radiation transport equation. Howelse, in many applications the medium is optically thin to emitted radiation, and the angle and frequency variables must be retained.

This paper describes a method of integrating the frequency iariable out of the transport equation when the medium contuins optically thin regions. The integration leaves a parameter called the radiation temperature to represent the irequency spe:trum, where the radiation temperature is chosen to reflect local and nonlocal emitting regions. The model is referred to as a two-temperature model besause both the local and radiation temperature appear in the equations. The equations are identical in form to those set forth by Moses and Peterson. ${ }^{1}$ but the dentation presented here sugpests a method of evaluatung the ratianon temperature that asolis problems assosiated with the previous two-temperature model. The model derived here is partucularly well suited to a medium composed of altemately iransparent and opatque layers, bui is an economiea! Fist estimat: for $3 n y$ applitition where net energy iransport is of rninsin interest. and a detalled desinption of the frequensi spectrum is not needed.

The thetempisature equations are derived in Sec. Il. Thermul conduction and motion of the nediun are ignored in that section because neither influencis the deriation. The equations are used in Sec. III to analytically study the phenomiena following the explosion of an inertial continement fusion (ICF) targer in a pas. The trends revealed 


\section{PROPAGATION OF IONS THROUGH CONDUCTING FLUIDS WITH IMBEDDED MAGNETIC FIELDS}

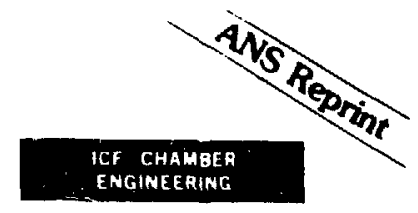

IHOR O. BOHACHEVSKY, JOHN C. GOLDSTEIN, and

DONALD O. DICKMAN Los Alamos National Laboratory

Analysis and A ssessment Duvision. P.O. Box 1663

Lus Alama: New Nexico 87945

Received May 24, 1982

Accepted for Publication January 14. 1983

\%

Studies of applications of inertial confinement fusion motivated the development of a plasma model composed of

1. perfectly conducting Juid

2. relatively energetic ions not in thermal equilibrium with the fluid

3. an electromagnetic field.

The fliid is modeled as a continuum, but the trajectories of the ions are determined from integra. tion of the equations of motion for a statistically representative sample of simulation particles because the ion ranges in the fluid are comparable to characteristic dimensions of containment vessels. The model constituerts interact electrodynamically. col. lisionally, and through iontation and recombination processes. The model of the collisional interaction is based on the stopping power of the fluid; it leads to rechnical difficulties because of the widely different characteristic lengths associated with collisional interactions and with macroscopic fuid phenoment. These difficulties are resolved with a specially constmucted elementary one-dimensional model of the collissonal interaction. The finite difference equetions describing the evolution of tire complete ion-piesme system are integrared numerically for an isotropic ion source located on the cylinder axis. The solutions indicate different ion behaviors for low- and highfisid densities. The ions expand as a diffuse cloud through low'density flutds, but aggregaie into perpendicular-to-the-magnetic-field sheets in high-density fiuids; the discovery of these strikjngly difjerent behaviors constituies the main contribution of this work.

NUCLEAR TECHNOLOGY/FLSION VOL. \& JULY 1983

\section{INTAODUCTION}

In the inertial confinement fusion (ICF) process, the reaction occurs sufficiently fast (in $<10^{-9}$ s) to resemble a small explosion (called a microexplosion) that produces relatively high-energy $(1-$ to $100-\mathrm{keV})$ ions. Investigations of the expansion of these ions and their interaction with an extemally generated magnetic fietd imbedded in a residual background fluid led to the study of a plasma model composed of

1. an ionized huid

2. relatively energetic ions not in themodynarnic equilibrium with the surrounding fluid

3. an electromagnetic field.

The assumptions implicit in this model are:

1. The background medium is collision-domi nated, behaves as an ideal gas with a constant ratio of specific heats, and is ionized to a degee at which its electrical resistivity may be neglected.

2. The discrete ions do not directly interact with each other and are not in thamodynamis equilibium with the sumounding fluid in which their ranges are comparable to the characteristic dimensions or interest

3. The interaztion forse between the ions and the surrounding fluid is always tangent to the ion trajectory, i.e., scattering is neglected.

4. Ar adequate number of suficiently mobile eiectrons are available to ensure tozal tharge neutrality and to short out any elecirostatic field.

5. The electric and magnetic fields are aluay's and everywhere perpendicular.

6. High-frequency phenomena are ignored, i.e., the displacement current is neglected. 


\section{CYCLIC TEMPERATURE AND THERMAL Gas STRESS FLUCTUATIONS IN FUSION REACTORS}

IHOR O. BOHACHEVSKY Los Alamos National Laboratury

P.O. Bax 1663. Los Alamos, New Mexico 87545

RONALD N. KOSTOFF* U.S. Department of Euerg'

Office of Energy Research, Washingion, D.C. 20545

Received December 30, 1981

Accepted for Publication May 4, 1982

\section{an}

Cyclic thermal loads and stresses in two critical componenis of fusion reactors. including fusionfission hybrids, are modeled and calculated The iwo critical components are the solid wall adjaccur to the fusion plasma ("first wall") and the fissile fucl elements in the high-power density region of the blanket. These two componenis exemplify two limiting cases of thermal loading: The first-w'all loads are generated 6!' predominantly shallow energy' deposition that may be approximated with a fiux across the surface and the fuel elements loads are generared by volumerric heating.

Two approaches are used to solve the hear conduction equation and to calculate the resulting siresses in rerms of sistem parameiers. The first is expansion into Founer senes and determinzision of periodic solusions: the second is analysis and superposition of single-pulse responses wetgri:ed with appropriate time delay and decay izetors. A pproximate closed-form expression for semperature excursions and thermal stresses are cicrised: inese expressions may be exalusted convenien:ly ard rapialy for comparison of differens sistems. The results provide a quantitarive basis for trade-off studies and comparative assessments of differen: fusion reactor sisiems.

\section{INTRODUCTION ANO SUMMARY}

A number of potential applications of fusion reactors have been identified and investigated several

The vews expressed in this paper are those of the author and
do not necessarily represent the vews of the Department of do not nece

NUCLEAR TECHNOLOGY/FLSION VOL. : OCT 198: that appear technically and economically feasible are production of electricity, process heat, synthetic fuels, and fissile fuels. Realization of these applications requires solution of many outstanding and challenging scientific and engineering problems. One of the Jutstanding problems for most fusion systems is the modeling and analysis of cyclic thermal stresses with a view toward raducing their effects on availability. reliability, and component lifetime.

From the mechanical engineering point of view. one fundamental difference between the fusion and fission reactors (including advanced fuel breeders) is the type of operation: Fission reactors operate in a steady state while fusion reactors, in general. are expected to operate clically. The cycle time or period may range from tens of minutes to small fractions of a second and the fraction of the cycle during whish fusion energy is released may range from neariy unity to $10^{-6}$ or less. Consequently, the starasteristics of cyclic thermal and mechanical lcads in fusion reastors will span a wide range of paranete: values.

In this paper we model and analyze cyclic thermal loais and stiesses in the complete potential range of operasing conditions. He examine two critical components of fusion reactors (including fusionfission hybrids): namely. the solid wall adjacent to the tisson giasma ("first wall") and the fuel eisments losated in the high-power density region of tae blanket. Trese iwo components exemplify iwo limiting cases of thermal loading: The firstuall loads I": gene:ated by piedominantly shallow eneagy depositicn tiat may be approximated with a flux across the surface and the fuel element loads are generated by precominantly volume energy deposition or volumetric heating. For these two types of loading, we derive approximate closed-form expressions for temperature increases and themal stresses that may be evaluated conveniently and rapidly for comparison of different systems. 


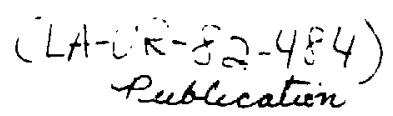

NLCLEAR SCIENCE AND ESGINLERIN: 82, 416-428 (1982)

\title{
Consistency of Neutron Cross-Section Data, $S_{N}$ Calculations, and Measured Tritium Production for a 14-MeV Neutron-Driven Sphere of Natural Lithium Deuteride
}

William A. Reupke* and D. W. Muir

Los Alamos National Laborarory, P.O. Box 1663

Los Alamos, Vew ilexico 87545

and

J. Narl Davidson

Genrgla Insmure of Technolagv School of Vuclear Enģineenng

Aliania, Georgta 30332

Received March 1. 1982

Accepted Julu S. 1982

\begin{abstract}
he Deesent algonthms, describe a compuie: progrzm, and give a computitionsl

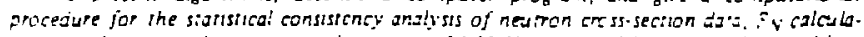
fiuns. and mecsured intam production in 14-1tel neutron irien integrai assemblies. figonthrs fresented inchude, reduced mainx manipulanon technoue wilabie for manygroup. 14.1feV nesiran iranspon calculations. The computer program inconpora:es these

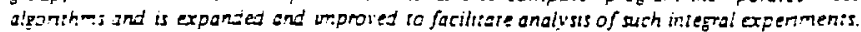
Detals of the comsurstoral procedure are given for a napural lithim deutente expenment

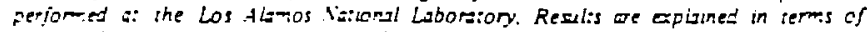

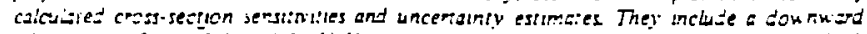

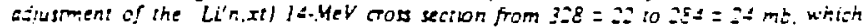

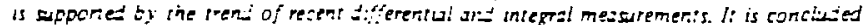

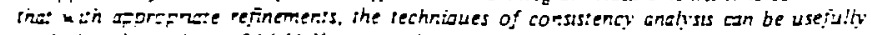

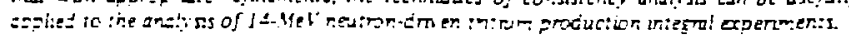

\section{INTRODLCTION}

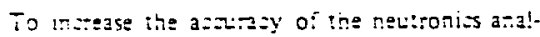

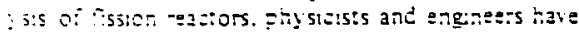

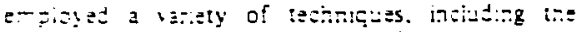

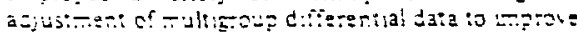

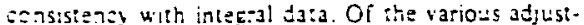
men: siralegles. a geriaralized least-squares procecius that adyusts the comouned difte:enial and integral C3:a san signinisantly improve the azeuracy of new-

-Present adjess RIPCO. $5: 5$ Cordova Ruad. Süle i:s Sania Fe. Meu MIaxiso S?5O!

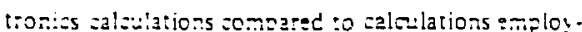

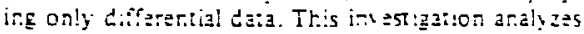

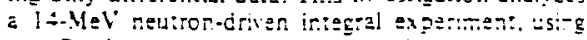
a refinad mettodelogy and a comired sensitiv:? ana!ysts and sons:stanty ana:ysts zode. to stend the

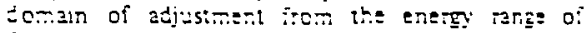
fisstor reacto:s to the energy range of deutenintritium fusion reactors.

In the higher snergy regime of the fusion reasto: several problems in the application of conststency analysis can be expezted. Because of the highes energes involved, a lorger nimber of zross-section energy groups are used. Moreover. $t^{2}$. zher erergy 


\title{
Power plant design for inertial confinement fusion: Implications for pellets *
}

\author{
T. G. Frank and J. H. Pendergrass
}

Las Alamos Not.onal Laboralon; Las Alamos. New Mexico 87s4s

O. L. Cook

Sondia Varional Laboraion. Albuquerque. New Mexico 87185

J. H. Pitts

Laurence Livermore National Labaratory. Livermore, California 94550

(Received II September 1981; accepted 19 Oc:ober 1981)

Potential commercial applications of inertial fusion include the production of elertricity and fissile and chemical fuels. In all these applications, a significant arzount of high-temperature heat will be produced and convered to electricity. Most of the implications for fusion pellets are common to all applications; the emphasis in this discussion is on central station electric power plants. The requirements and constraints on fusion pellets for power plant applicalions include: survivability in hostile reaction chamber environments, the ability to withstand large acceleration forces encountered in high-velocity injection, suficiently large energy releases for economic power production, and high-rate automated manufacture at costs whch are not a large fraction of the value of the fusion energy released.

PACS numbers: 28.50. Re, $52.50 . J \mathrm{~m}$

\section{INTRODUCTION}

Potential commercial applications of inertial fusion include the production of electricity and fissile and chemical fuels. In all these applications, a significant amount of high-temperature heat will be produced and converted to electricity. Because most of the implications for fusion pellets are common to all applications, the emphasis in this discussion is on cen. iral station electric power plants.

In an inerial fusion electrie power plant, fusion pellets concuning deuterium and tritium (DT) will be injected into one or more reaction chambers at rates of $1-10 / \mathrm{s}$ at high velocity w'bere they are intercepted in flight, by laser or parvicle beam driver pulses. The fusion fueld is compressed and beared to conditions necessary for fusion reactions to take place Energy is reieased from the pellet as $x$ rays, inizh-energy seutrons and enerzeric peliet debris The $x$ rays and pellet debris ions deposit their energi very neas surfaces of incidence, whereas neutron energy deposition ocsurs volumetrically in relatively thick blanket regions surrounding the reastion chambers. Special provisions are required to acsommociate the $x$-ray and pellet-debris energy deposition zithout severe damage to the interior surfaces of reaction chambers of ieasonabie size. Pellet energy reieases are converted to sensible beat in reactor struetures and coolants and are transported by a fooring cooiant to appropriate power conversion equipment.

The requiremants and constraints on fusion pellets for power plant applications include: survivability in hostile reaction chamber environments, the ability to withstand large acceieration forces encuuntered in high-telocity injection, sufficiently large energy releases for economic power production. and high-rate automated manufacture at costs

- Published without author corrections. which are not a large fraction of the value of the fusion enerby released. These raquirements and their dependence on the several possible conibinations of generic reactor-dnver combinations are discussed in this paper.

\section{PELLET CONCEPTS}

The basic concept for producing energy from fusion pellets is illustrated in Fig. 1. Driver energy is delivered io a fusion pellet by iniense, shor-pulse laser or paricle beams. An outer layer of the pellet is heated very rapidly causing it to expand with high velocity. The fusion fuel is compressed and heated. due to the recoil impulse of the expanding material, to high density 11000 to 10000 times liquid density and temperature with ignirion oceurring at $3-5 \mathrm{keV}$. The thermonuclear burn spreads rapidly through the compressed fuel releasing many times the driver input energy.

At the present time, researsh is being conducted to establish the rechnical feasibility of the basie process through singie-shot physies and peller expeciments using high-energy lasers and light-ion-beam azeelezaro:s. Comprehensive re. views of the experimental programs are given in Refs. $I$ and 2. Studies of conceptual desizns of inerual fusion pouer plants are based on theoretucal fusion pelies designs japable of fusion gains lfusion energ: o::?ut divided by driver energy input to the pelietı ranging from approximately 50 to sev. eral hundred with driver pulse enerzies in the $2-10 \mathrm{MJ}$ range. Although peilet designs appropriare fos povier plant afplication have not yet been demonstrated, arork to date does permit projections to be made on design and quality control requirements. 'Figure 2 shows the general features of several generic designs of fusion pellets. In adition to the designs shown, peliets having multiple fuel regions are being 


\section{PATENTS \\ SINCE}

1982 


\begin{tabular}{|c|c|c|c|}
\hline $\begin{array}{l}\text { United States Patent [19] } \\
\text { Duchane et al. }\end{array}$ & $\begin{array}{l}\text { [11] } \\
\text { [45] }\end{array}$ & $\begin{array}{l}\text { Patent Number: } \\
\text { Date of Patent: }\end{array}$ & $\begin{array}{r}4,481,999 \\
\text { Nov. 13, } 1984\end{array}$ \\
\hline
\end{tabular}

[94] METHOD OF FORMING A THIN UNBACKED METAL FOIL

[73] Inventors: David V. Dectave, Los Alamos: Barry L. Bartiell, Teuque, both of N. Mex.

[73] Assugnee: The Ualted States of America ma reverested by the United States Depertweat of Enerz. Wishington. D.C.

[2!] Appl. No.: 351,378

[22] Filed: Fet. 23, 1942

[51] Int. CL' ................ B22C 7/02; $822 \mathrm{C} 9 / 24$. B22D 23/00, B22D 29.'00

[52] US. C1 . 164/6; 164/36 164/44: 160/45: 164/46; 164/131, 104/138 [58] Field of Search . ............. 164/6. 14, 19, 23, 33, $164 / 44,45,46,47,72,74,131,132,138,36$

\section{Referesces Clied}

U.S. PATENT DOCUMENTS

1.47.683 3/1923 Morns

$1.889 .90512 / 1932$ Seger

318909 s/1963 seger

3.181 .209 s/9.6\% same

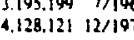

FOREIGN PATENT DOCUMENTS

$14659083 / 1977$ Unted Kingdom
697244 II/1979 U.S.S.R

$164 / 45$

\section{OTHER PUBLICATIONS}

Kindel, F. W. et al., "Thin Foil Fabrication," in Review: of Scientific Imsruments vol. S0, No. 12. Dec. 1979, pp 1550-1532.

Springer. R. W. et al.. "Siructure and Mechanical Properties of $\mathrm{Al} / \mathrm{Al}_{\mathbf{r}} \mathrm{O}$, Vecuum Deponited Laminates," in Thin Sovid Films, vol. S4, 1978, pp. 197-203.

P. imary Examiner-Kuang $Y$. Lin

Assiston: Examiner-J. Reed Batten, ir

Attomey, Agent, or Firm-William A. EkJund; Paul D Getjens; Micheel F. Eaposito

[57]

AESTRACT

In a inethod of forming a thin $(<2 \mu m)$ unbacked metal foil having a desired curviplannr shape, a soluble poly. meric film. preferably comprising polyvinyl alcohol. is formed on a supporing structure having a shape :hat defines the dasired shape of the foil product. A layer of metal foil is deposited onto one side of the soluble film. preferably by vacuum vapor deposition. The melallized film is then immersed in a suitable solvent to dissolve the film and thereby lesve the metal foil as an unbacked metal foil element mounted on the supporting structuie. Aluminum foils less than $0.2 \mu \mathrm{m}(2,000 \dot{A})$ thick and having an areal density of less then $34 \mu \mathrm{g} / \mathrm{cm}^{2}$ have been obtuned.

2 Calms, 3 Draviag Figures

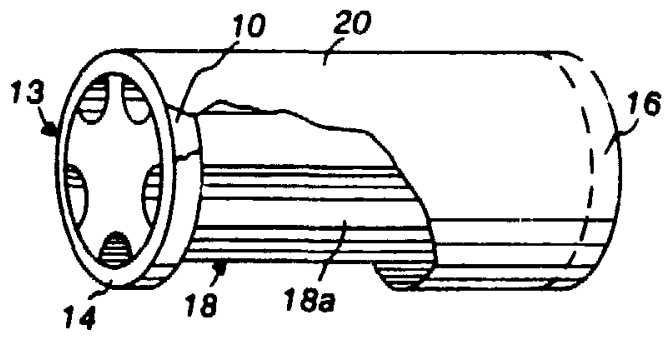


[54] COMPOSTE ROLYMERIC FTM AND METHOD FOR TTS USE IN INSTALLING A VERY THIN POL YMERIC FILM IN A DEVICE

(75) Inventors: Darld V. Duchenes, Los Alnomos Berry L. Bartbell, Tesuque, both of N. Mex.

[73] Astignee:

To Ualtad Stite of Anerton a reposated by the Uplted States Departonet of Eserd, Washington. D.C.

[21] Appl. No.: 371.749

(22) Filed: A.r. 26, 1992

[si] lot C2!

[32] U.S. C2 BO2B 31/00, B32B 27/42 156/659; 156/668; 426/213; 428/501; 428/503; 428/507; 428/5C8; $428 / 515 ; 428 / 520 ;+28 / 524$ $428 / 526 ; 428 / 534 ; 428 / 914$

[58] Field of Serret .............. 428/213, 515, 520, 501 428/503, 508, 524, 526; 525/61; 428/411, 507,

[56] 428. 534, 914; 156/155, 230, 655, 668

Reforeses Claed U.S. PATENT DOCUMENTS

3,484,409 12/1969 Ashikage et al. ..... $929 / 61$

\begin{tabular}{|c|c|c|}
\hline $\begin{array}{l}3,505,264 \\
3,859,125 \\
4,119,604 \\
4,372.311\end{array}$ & $\begin{array}{r}1 / 1970 \\
1 / 1975 \\
10 / 1978 \\
1 / 1983\end{array}$ & 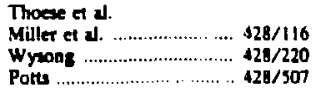 \\
\hline
\end{tabular}
$4,372.311$ V/1983 Ports ................................. 428/507

4,379,205 4/1993 Dowang at 4) ................ 929/6

Primary Examiner-George F. Lesmes

Assurant Examiner-E. Rollins 8uffalow

Attomey, Agent or Firm-Willinm A. Eklund; Paul D. Gaetjens

[57]

\section{ARSTRACT}

A composite polymeric film and a method for its use in forming and installing $a$ very thin $(<10 \mu m)$ polymetic film are dieclosed. The composite film consists of a thin film layer and a backing layer. The backing layer ts soluble in a solvent in which the thin film layer ss not soluble. In eccordance with the method, the composite film is installed in a device in the sume position in which it is sought to finally emplace the thin film. The backing layer is then selectively diseolved in the solvent to leave the insoluble thin film layer as an unbacked film. The method permits a very thin film to be successfully instaled in devices where the fragility of the film would preclude handling and installation by conventional methods.

14 Craime No Drawn 


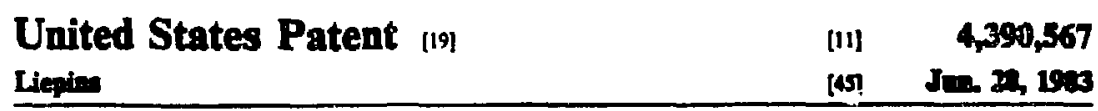

[S4] METHOD OP PORMING GUADT

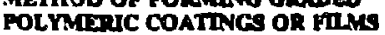

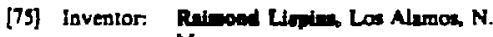
Mex.

[73] Assignes: Du Uaited State of Anviles

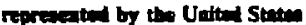
Depranent of Exere, Wishingron. D.C.

[21] Appl. No.: 242807

[22] Filed: Mr. 11, 1991

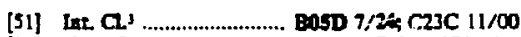

[s2] US. Q 376/152; 376/916; 427/6; 427/215; 427/217; 427/221: 427/222; 427/250; 427/252; $427 / 255.6 ; 427 / 255.7$

[58] Field of Search .................... 376/191, 192, 916; $427 / 250,252,38,39,6,40,41,213,214,215$, Referesces CTted U.S. PATENT DOCUMENTS

$2984535 / 1961$ Fitch $3.163 .66512 / 1964$ Fitch $106 / 1$

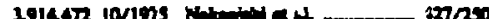

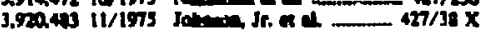
3,994,022 11;1976 De Ened a t. .............. 427/6 X 4.123,30: 10/19m Nowith a al ......................... 427/41 4,224,26t 9/19e0 Hulpent........................ J76/152 X 4290,447 9/1981 Johmen ai . $427 i d X$

Primor Exominop-Notmian Mortenters

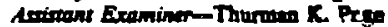

[57] ABSIRACT

Very swooth polymeric conting or tim poded in aromic number and dentivy cen resty be formed by first preparing the coctins or flly from the desired monolueric wateritl and thea couracedoe it with a fluid contrining a detal or a mixture of metaly for a time sufficient for such wetal or methls to sorb und diffues isto the conting or film. Metel revinete colutions are particularly adventageous for this purpose $\mathbf{A}$ metallic conting can in turn be produced on the wetel-landed film or conting by exposine is to s low presure planen of air, oxyger, or nitrous oxides. The process permits a metallic costing to be formed on a beas sencitive substrate without the use of elevated temperarurea.

14 Clater, No Dewioge 
[54] PRODUCTION OF SUPER-SMCOTH ARTICLES

[75] Inventor: Darid Y. Ductand, Los Alamos. N Mex

[73] Assignee: The Uatted Seale of Amerien as represeated by the Departmeat of Energo, Washingion. D.C.

[21] Appl. No.: 268,423

(22) Filed: May 29, 1911

[51] Int. C1. ' .................. Bac 25/00

[52] U.S. ¿. . ............................ $264 / 341 ; 428 / 409$

[SB] Field of Searcb ………........ 20\$/341, 423/409

References Cited

U S PATENT DOCUMENTS

2.200.940 7:1940 Smith

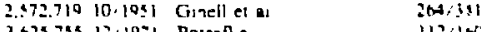

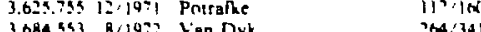

3.684 .553 8/19:2 Ven Dyk

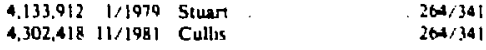

$26+134$

OTHER PUBLICATIONS

Chemical Abstracts, vol 82. 1075. pp. 43-44. No $98967 \mathrm{~m}$
Chemical Abstracts, vol. 83, 1975, p 83, No 115655, Chemical Abstracts, vol. 90. 1979. p 53. No 90 169698c

Chemical Abstracts, vol. 84, 1976, p. 50, Nc 84:606589 Daniels et al. Physical Chemustry. 2nd Evition. Wiley and Sons, New York, 1963. p. 354

Modern Plastics Encyclopedia, pp. 5:33-536. Gross Ednot-1n-Chief (McGraw-Hill. N\} 1974-1975)

Priman Examiner-James H. Derrington

Attorney. Agent. or Firm-Elizabeth O. Slade; Paul D Gactjens; Richard G. Besha

[57]

ABSTRACT

Super-smooth rounded or formed articles made of thermoplastic materals including varous polyimethyl methacrylate) or acrylonitrile-butadiene-styrene copolyrners are produced by immersing the aricles into a bath. the composition of which is slowly changed with time. The starung composition of the bath is made up of a) leasi one solvent for the polymer and a diluent made up of at least one nonsolveht for the polymer and op. tional materials which are soluble in the bath. The resulturg exiremely smooth aricles are useful as mandrels for laser fusion and should the useful for a wide varety of other purposes, for example lenses

12 Claims. 6 Draming Figures 
[S] COATEZ WOVEN MATEAIALS AND METHOD OF PREPARATION

[15) Inventors: Willing J. Mocreary; David w Cerroll, both of Los Alumos, N. Mex.

[73) Amigner. The United Stuter of Anerles as newneated by the United Stutes Doperient of Eerts. Washington, D.C.

[21] Appl. No.: 47,445

[22) Filod:

Jar. 11, 1978

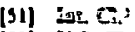

[s2] U.S. C.

BOSD 1/22; B324 7/08

(58) Fied of Siearch .............. 427/213, 243, 244, 245.

[56]

$427 / 247,249,291 ; 428 / 263,608$

\section{Referesen Citad}

\section{U.S. W'ATENT DOCUMENTS}

3,178.305 4/196s Odley.et al. ........................ 427/213

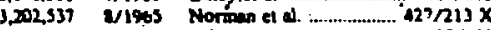

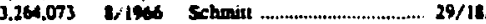

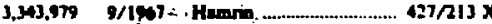

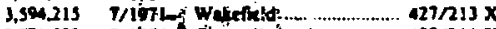

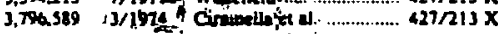

4.071.J04 1/1978 Cheuvin at al ............... +27/213 $X$ 4000.927 y/1988 Brown.

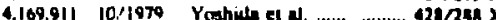
4.110.428 12/1979 Rute et al. ..................... 421240 $x$

OTHER PUELICATIONS

Powell et al., "The Formation of Metallic Cantings by Yapor Phese Techniques", Metal Finistine vol. sn. No. 4. Apr. 1952, pp. 64-69.

Primary Examinep-James R. Hofrman

.tlorney. Agent or Firm-Elizabeth O. Slade; Paul D Geetyens; Ruchurd G. Beshu

[37] ABSTRACT

Conting of woven materials so that not only the outer surfaces are cauted has been a problers. Now, a solution to that problem is the following; Woven melerials wse conted with materials, for ezample with metnlis or with pyrolytic citon, which msterinis are deporited in Chemical Vapor Deposition (CVD) reactious using a nuidized bed so that the porosity of the woven mulerial is recuined and so that the tin'y filaments which make up the urnads which we woven (including inner as well at oulet filaments) are abbetentinlly uniformly conted.

22 Calme, 11 Dratiat Firare

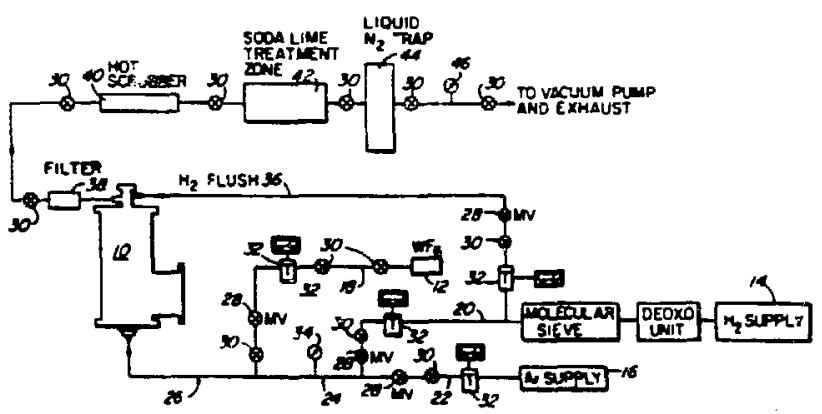

